\title{
Disilyl Complexes of Zirconium, Hafnium and Tantalum. Their Synthesis, Characterization and Exchanges with Silyl Anions
}

He Qiu, Hu Cai, Jaime B. Woods, Zhongzhi Wu, Tianniu Chen, Xianghua Yu, Zi-Ling Xue* Department of Chemistry, The University of Tennessee, Knoxville, Tennessee 37996

\section{Supporting Information}




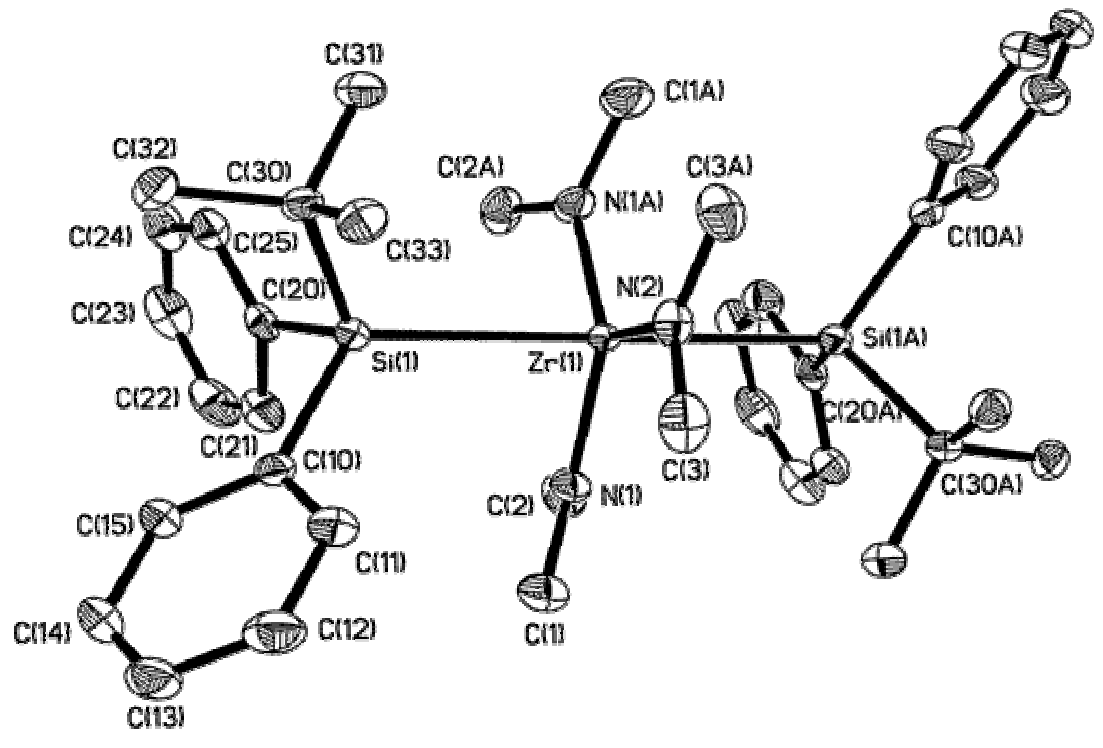

Figure S1. An ORTEP view of $\left(\mathrm{Me}_{2} \mathrm{~N}\right)_{3} \mathrm{Zr}\left(\mathrm{SiBu}^{t} \mathrm{Ph}_{2}\right)_{2}{ }^{-}$(1) showing 30\% probability thermal ellipsoids. The $\mathrm{H}$ atoms have been omitted for clarity. The structure of this complex is isomorphous to that of its Hf analog 2 (Figure 2). 


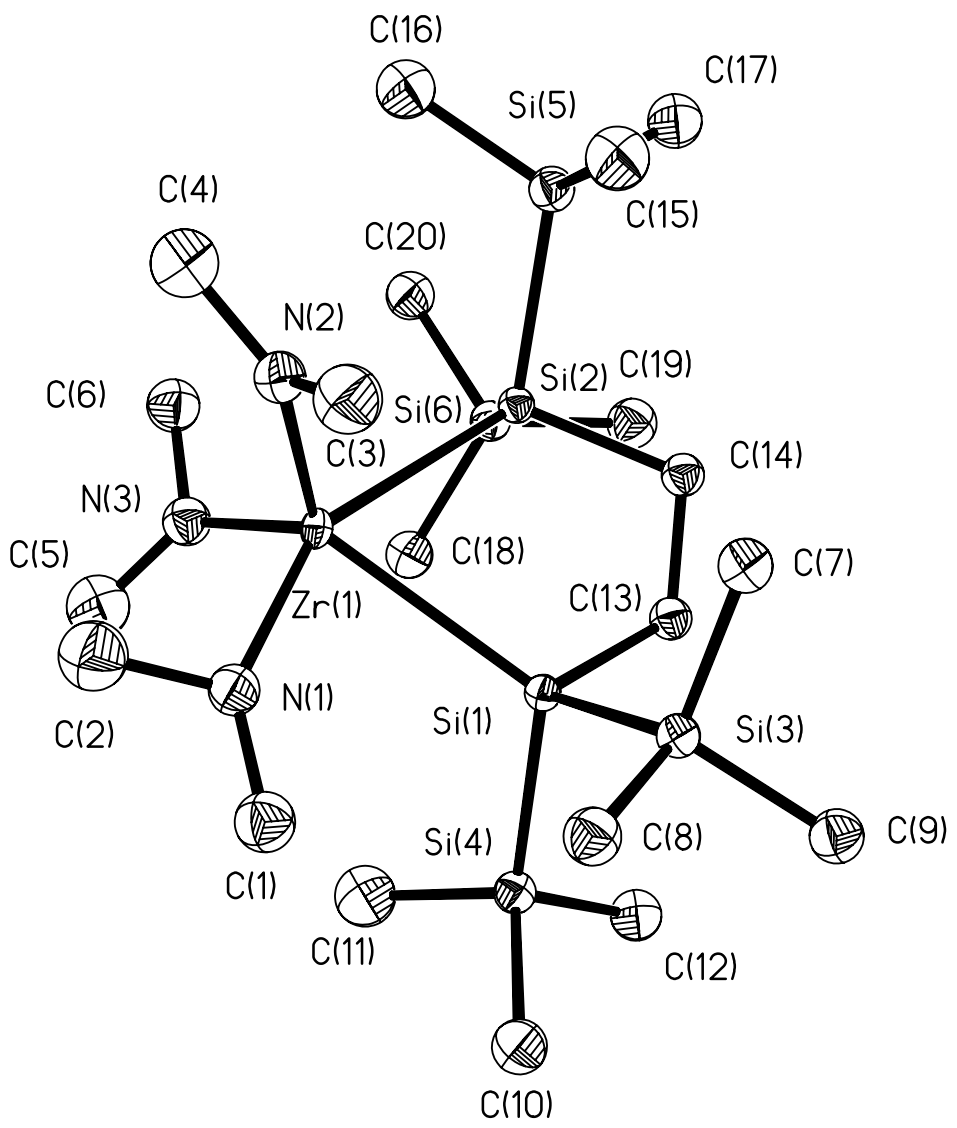

Figure S2. An ORTEP view of $\left(\mathrm{Me}_{2} \mathrm{~N}\right)_{3} \mathrm{Zr}\left[\eta^{2}-\left(\mathrm{Me}_{3} \mathrm{Si}\right)_{2} \mathrm{Si}\left(\mathrm{CH}_{2}\right)_{2} \mathrm{Si}\left(\mathrm{SiMe}_{3}\right)_{2}(3)\right.$ showing 30\% probability thermal ellipsoids. The $\mathrm{H}$ atoms have been omitted for clarity. The structure of this complex is isomorphous to that of its Hf analog 4 (Figure 3). 

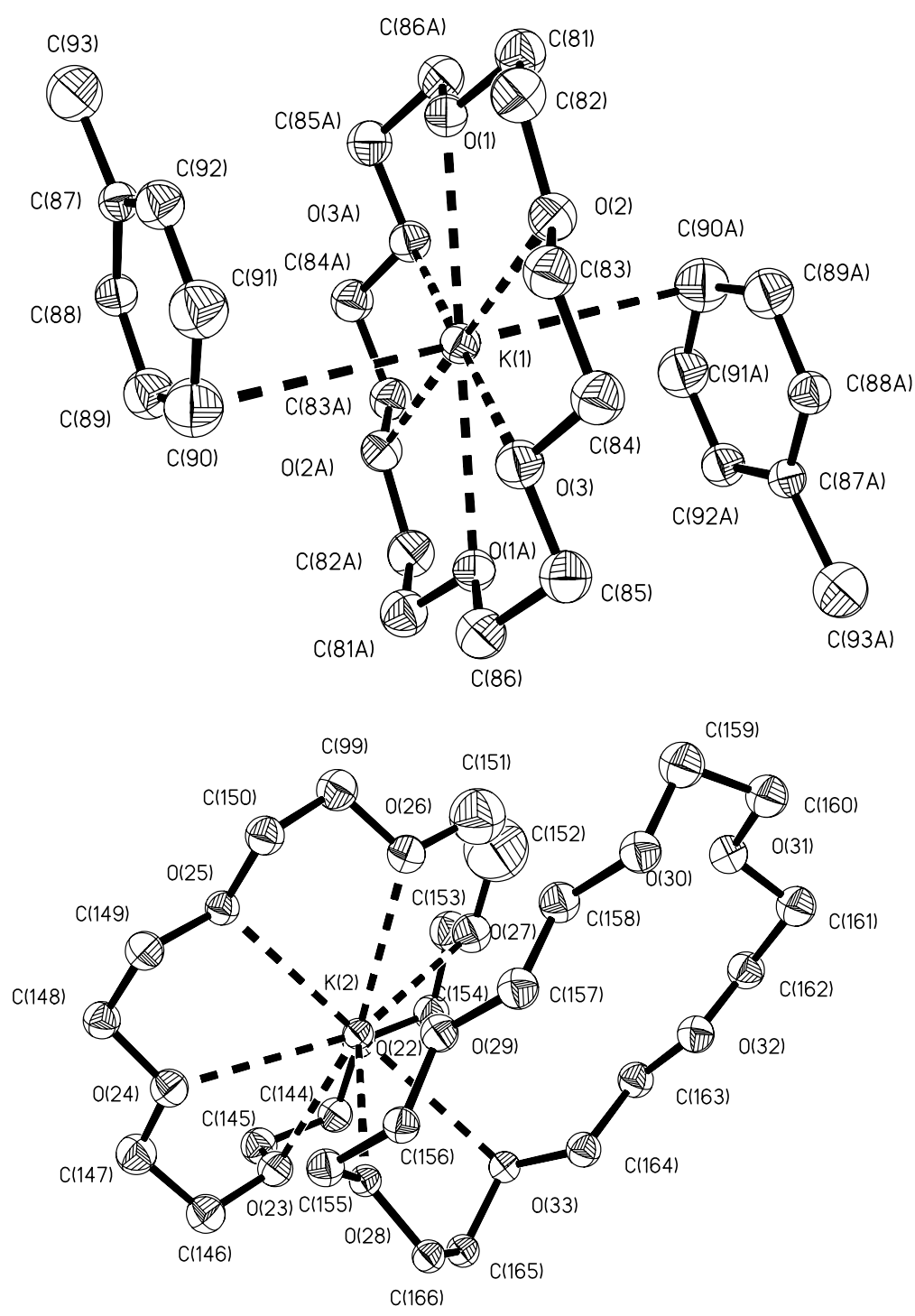

Figure S3. An ORTEP view of cationic K(18-crown-6) ${ }^{+}$portions of 3 showing $30 \%$ probability thermal ellipsoids. The $\mathrm{H}$ atoms have been omitted for clarity. The structure of this complex is isomorphous to that of its Hf analog 4 (Figure 4). 


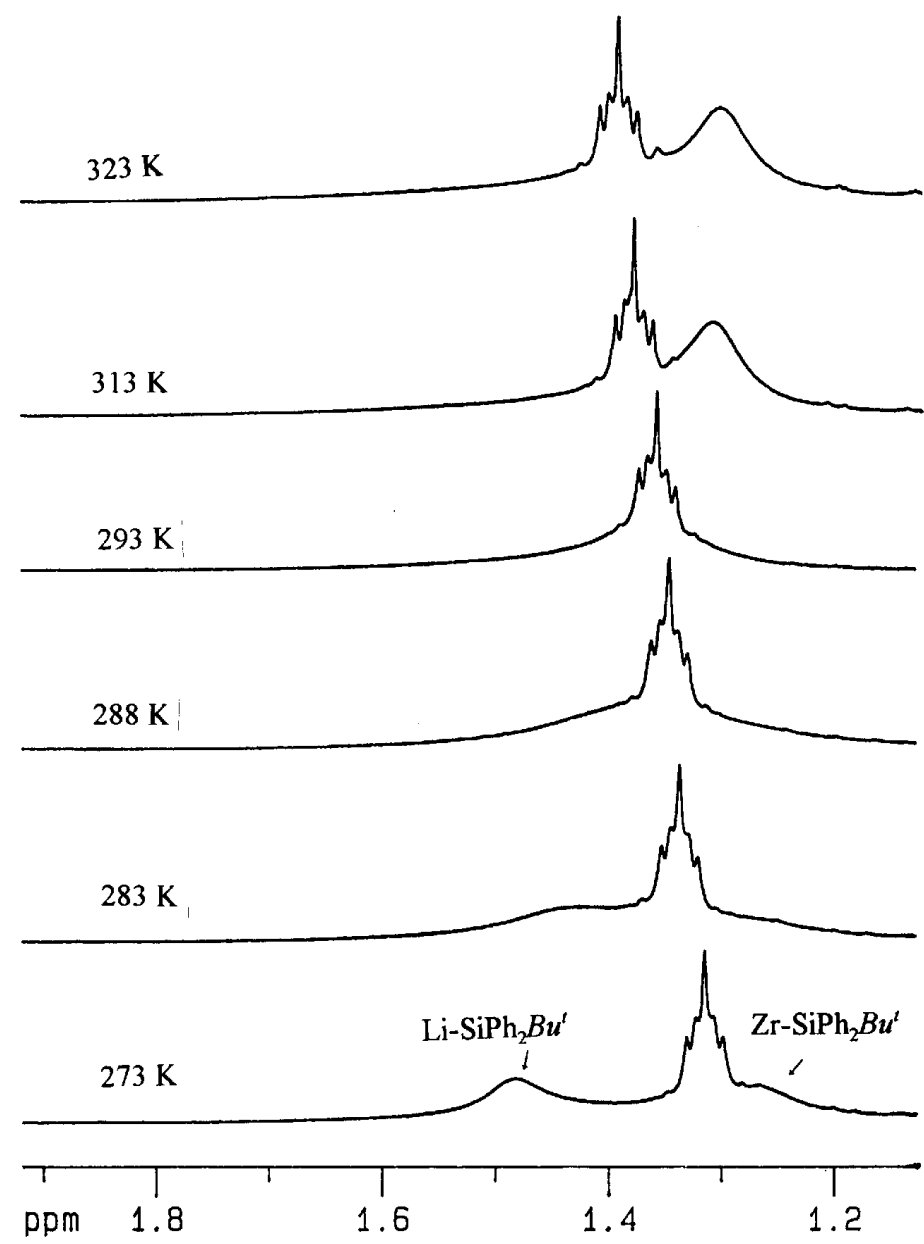

Figure S4. Dynamic ${ }^{1} \mathrm{H}$ NMR spectra of $\mathbf{1}$ in toluene- $d_{8}$. 


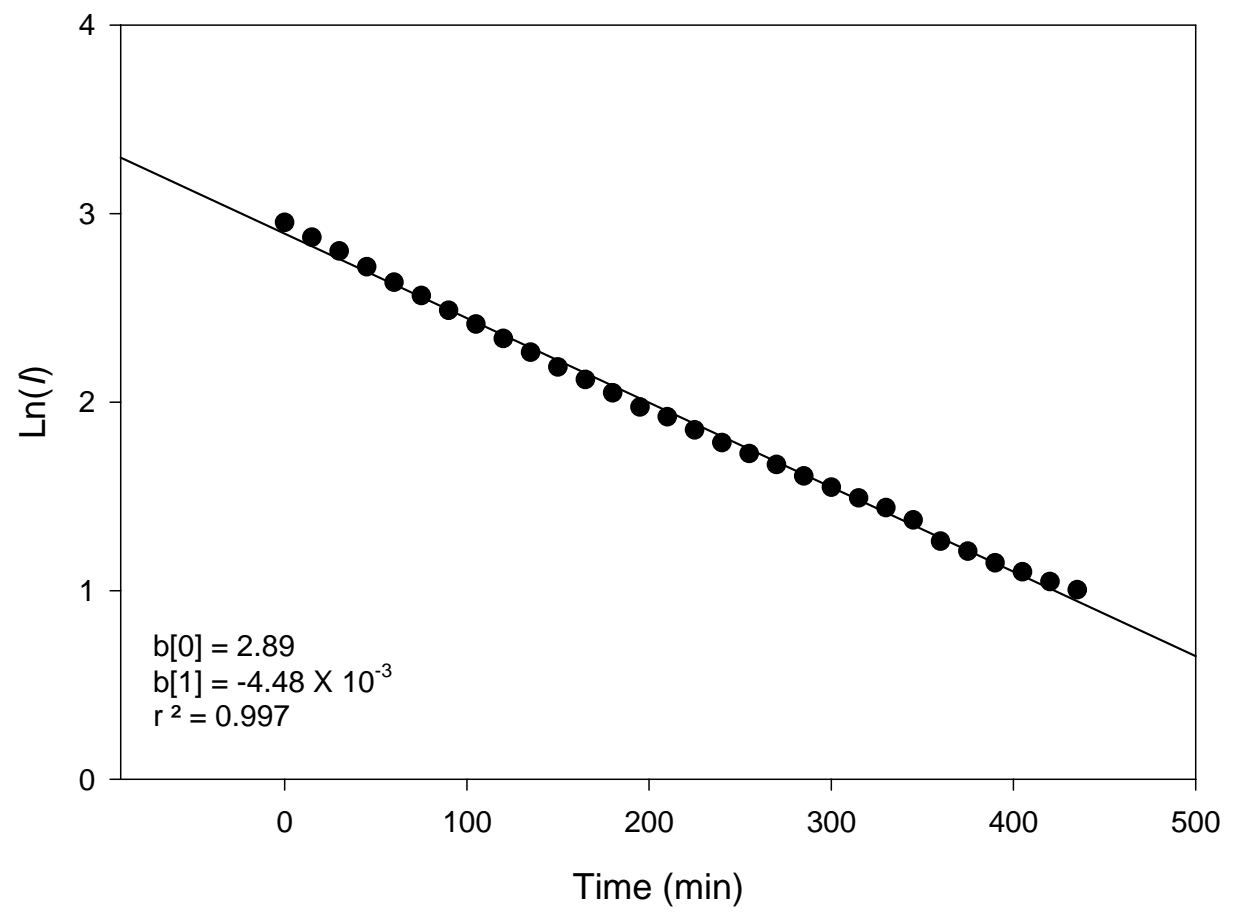

Figure S5. Kinetic plot of the thermal decomposition of $\left(\mathrm{Me}_{2} \mathrm{~N}\right)_{3} \mathrm{Ta}\left(\mathrm{SiBu}^{\mathrm{t}} \mathrm{Ph}_{2}\right)_{2}(6)$ at $303 \mathrm{~K}$ in toluene- $d_{8}$. 


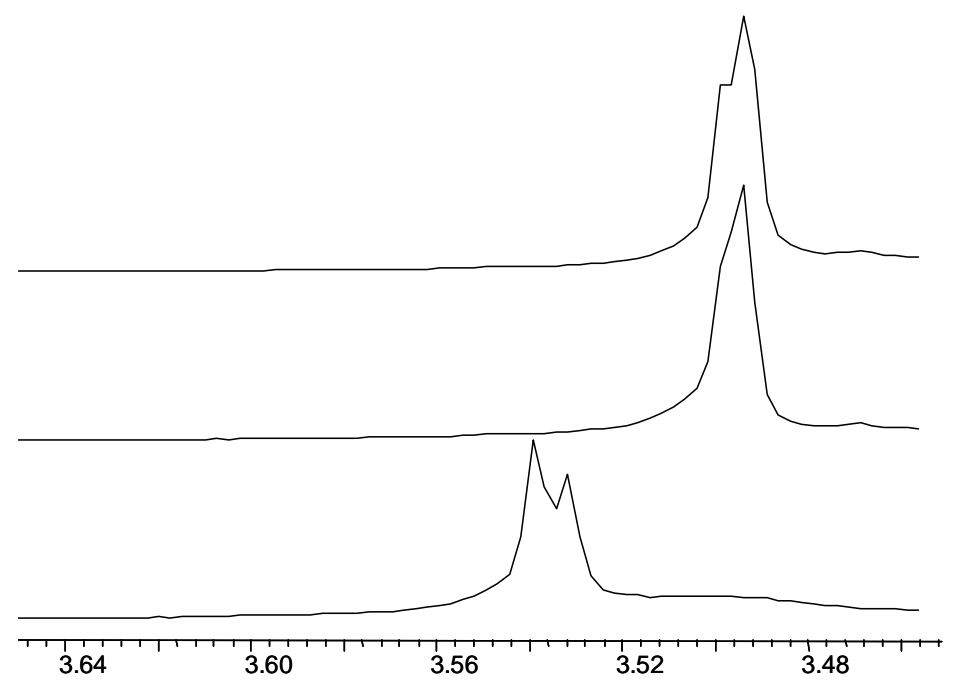

Figure S6. ${ }^{1} \mathrm{H} \mathrm{NMR}$ (at $400 \mathrm{MHz}$ ) of the $-\mathrm{NMe} e_{2}$ region of 3 at $20{ }^{\circ} \mathrm{C}$ (top), $10{ }^{\circ} \mathrm{C}$ and $-15{ }^{\circ} \mathrm{C}$ (bottom).

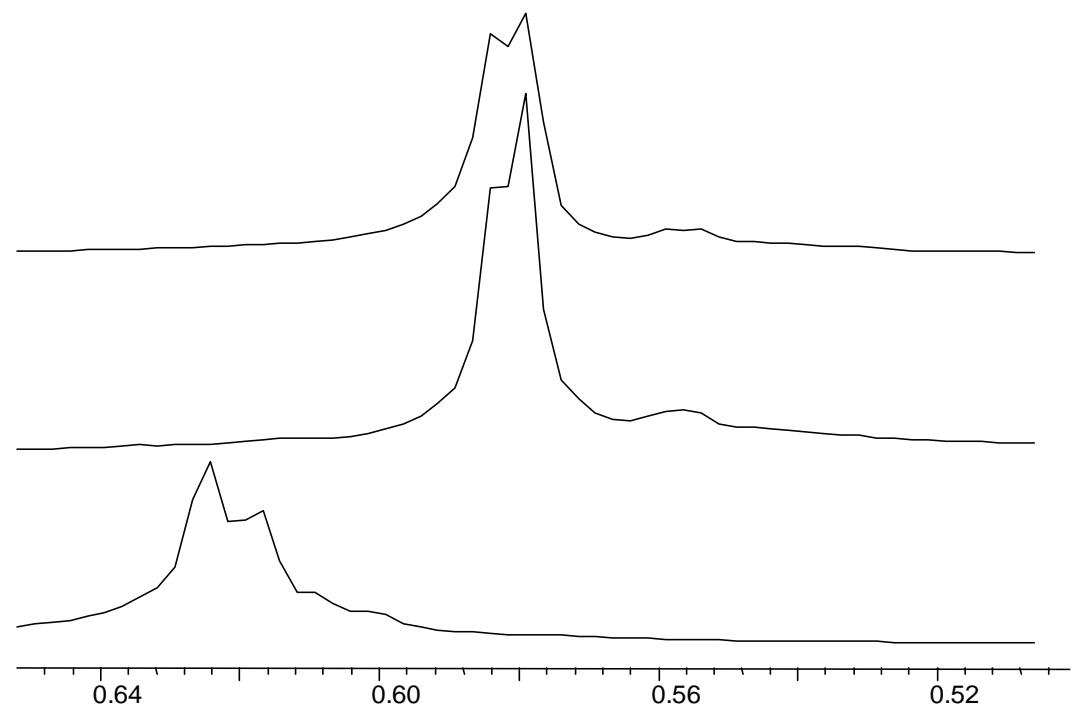

Figure S7. ${ }^{1} \mathrm{H} \mathrm{NMR}$ (at $400 \mathrm{MHz}$ ) of the $-\mathrm{SiMe} e_{3}$ region of 3 at $20{ }^{\circ} \mathrm{C}$ (top), $10{ }^{\circ} \mathrm{C}$ and $-15^{\circ} \mathrm{C}$ (bottom). 

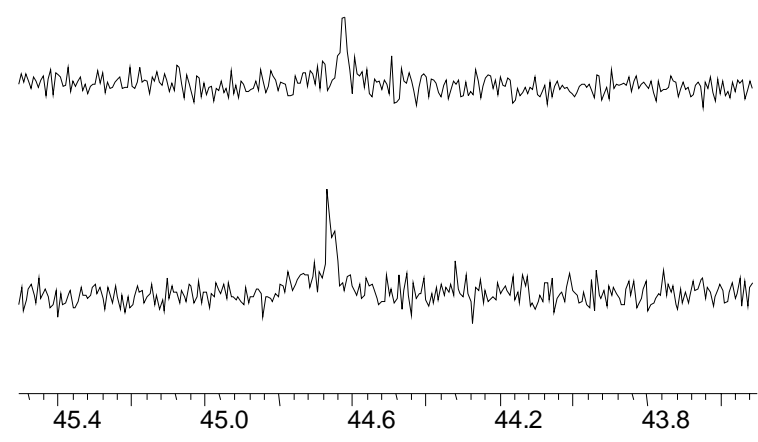

Figure S8. ${ }^{13} \mathrm{C}\left\{{ }^{1} \mathrm{H}\right\}$ NMR spectrum of the $-\mathrm{NMe} e_{2}$ region of 3 at $20{ }^{\circ} \mathrm{C}$ (top) and $-15{ }^{\circ} \mathrm{C}$ (bottom). 
Table S1. Crystal data and structure refinement for $\mathbf{1}$.

\begin{tabular}{|c|c|}
\hline Identification code & {$\left[\mathrm{Li}(\mathrm{THF})_{4}\right]\left[\left(\mathrm{Me}_{2} \mathrm{~N}\right)_{3} \mathrm{Zr}\left(\mathrm{SiPh}_{2} \mathrm{Bu}^{\mathrm{t}}\right)_{2}\right]$} \\
\hline Empirical formula & $\mathrm{C}_{54} \mathrm{H}_{88} \mathrm{LiN}_{3} \mathrm{O}_{4} \mathrm{Si}_{2} \mathrm{Zr}$ \\
\hline Formula weight & 997.61 \\
\hline Temperature & $173(2) \mathrm{K}$ \\
\hline Wavelength & $0.71073 \AA$ \\
\hline Crystal system & Monoclinic \\
\hline Space group & $\mathrm{C} 2 / \mathrm{c}$ \\
\hline Unit cell dimensions & $\begin{array}{ll}a=21.195(6) \AA & \text { alpha }=90^{\circ} \\
b=17.434(5) \AA & \text { beta }=119.76(2)^{\circ} \\
c=17.801(5) \AA & \text { gamma }=90^{\circ}\end{array}$ \\
\hline Volume, $\mathrm{z}$ & $5710(3) \AA^{3}, 4$ \\
\hline Density (calculated) & $1.160 \mathrm{Mg} / \mathrm{m}^{3}$ \\
\hline Absorption coefficient & $0.277 \mathrm{~mm}^{-1}$ \\
\hline$F(000)$ & 2144 \\
\hline $\begin{array}{l}\text { Crystal size } \\
\text { range for data collection }\end{array}$ & $\begin{array}{l}0.64 \times 0.62 \times 0.40 \mathrm{~mm} \\
1.61 \text { to } 22.54^{\circ}\end{array}$ \\
\hline Limiting indices & $0 \leq h \leq 22,0 \leq k \leq 18,-19 \leq 1 \leq 16$ \\
\hline Reflections collected & 3880 \\
\hline Independent reflections & $3761\left(\mathrm{R}_{\text {int }}=0.0256\right)$ \\
\hline Absorption correction & Semi-empirical from psi-scans \\
\hline Max. and min. transmission & 1.0000 and 0.7589 \\
\hline Refinement method & Ful1-matrix least-squares on $\mathrm{F}^{2}$ \\
\hline Data / restraints / parameters & $3758 / 0 / 295$ \\
\hline Goodness-of-fit on $F^{2}$ & 1.083 \\
\hline Final $R$ indices $[I>2 \sigma(I)]$ & $R 1=0.0465, \quad w R 2=0.1240$ \\
\hline$R$ indices (all data) & $\mathrm{R} 1=0.0622, \quad \mathrm{wR} 2=0.1464$ \\
\hline Largest diff. peak and hole & 0.525 and $-0.386 \mathrm{e}^{-3}$ \\
\hline
\end{tabular}


Table S2. Atomic coordinates $\left(\times 10^{4}\right)$ and equivalent isotropic displacement parameters $\left(\AA^{2} \times\right.$ $10^{3}$ ) for $\mathbf{1}$. $\mathrm{U}(\mathrm{eq})$ is defined as one third of the trace of the orthogonalized $\mathrm{U}^{\mathrm{ij}}$ tensor.

\begin{tabular}{|c|c|c|c|c|}
\hline & $\mathbf{x}$ & $\mathrm{y}$ & $\mathbf{z}$ & $\mathrm{U}(\mathrm{eq})$ \\
\hline $\operatorname{Zr}(1)$ & 5000 & $3031(1)$ & 7500 & $23(1)$ \\
\hline Si(1) & $3626(1)$ & $3001(1)$ & $7523(1)$ & $28(1)$ \\
\hline $\mathrm{N}(1)$ & $5455(2)$ & $2431(2)$ & $8632(2)$ & $40(1)$ \\
\hline$N(2)$ & 5000 & $4214(3)$ & 7500 & $37(1)$ \\
\hline$o(1)$ & $4453(2)$ & $1311(2)$ & $11505(3)$ & $76(1)$ \\
\hline$O(2)$ & $5603(2)$ & $2579(2)$ & $12251(2)$ & $56(1)$ \\
\hline Li & 5000 & $1946(7)$ & 12500 & $53(3)$ \\
\hline$C(1)$ & $5562(3)$ & $2686(4)$ & $9457(3)$ & $59(2)$ \\
\hline$c(2)$ & $5678(3)$ & $1638(3)$ & $8682(3)$ & $55(1)$ \\
\hline$c(3)$ & $5211(3)$ & $4683(3)$ & $8255(4)$ & $51(1)$ \\
\hline$c(10)$ & $3590(2)$ & $3286(3)$ & $8551(3)$ & $33(1)$ \\
\hline$c(11)$ & $3912(2)$ & $3963(3)$ & $8975(3)$ & $43(1)$ \\
\hline$c(12)$ & $3859(3)$ & $4234(3)$ & $9677(3)$ & $54(1)$ \\
\hline$c(13)$ & $3487(3)$ & $3815(4)$ & $9984(3)$ & $59(2)$ \\
\hline$c(14)$ & $3181(3)$ & $3135(4)$ & $9601(3)$ & $61(2)$ \\
\hline$G(15)$ & $3227(2)$ & $2870(3)$ & $8891(3)$ & $47(1)$ \\
\hline$c(20)$ & $3289(2)$ & $1958(2)$ & $7343(3)$ & $34(1)$ \\
\hline$c(21)$ & $3680(3)$ & $1431(3)$ & $8008(3)$ & $47(1)$ \\
\hline$c(22)$ & $3511(3)$ & $644(3)$ & $7891(4)$ & $60(2)$ \\
\hline$c(23)$ & $2958(3)$ & $376(3)$ & $7112(4)$ & $60(2)$ \\
\hline$c(24)$ & $2573(3)$ & $878(3)$ & $6452(4)$ & $50(1)$ \\
\hline$C(25)$ & $2737(3)$ & $1646(3)$ & $6568(3)$ & $40(1)$ \\
\hline$c(30)$ & $2858(2)$ & $3631(2)$ & $6645(3)$ & $31(1)$ \\
\hline$c(31)$ & $2819(2)$ & $3501(3)$ & $5772(3)$ & 41(1) \\
\hline$c(32)$ & $2106(2)$ & $3505(3)$ & $6556(3)$ & $40(1)$ \\
\hline$c(33)$ & $3059(2)$ & $4483(2)$ & $6879(3)$ & $39(1)$ \\
\hline$c(41)$ & $4601(8)$ & $1144(8)$ & $10869(9)$ & $238(9)$ \\
\hline$c(42)$ & $4197(9)$ & $523(7)$ & $10376(7)$ & $199(7)$ \\
\hline$c(43)$ & $3604(6)$ & $530(8)$ & $10511(9)$ & $212(8)$ \\
\hline$c(44)$ & $3875(6)$ & $869(7)$ & $11324(8)$ & $181(6)$ \\
\hline$c(51)$ & $5319(4)$ & $3128(4)$ & $11557(4)$ & $84(2)$ \\
\hline$c(52)$ & $5942(3)$ & $3539(3)$ & $11601(4)$ & $63(2)$ \\
\hline$c(53)$ & $6492(3)$ & $3515(3)$ & $12546(4)$ & $64(2)$ \\
\hline$c(54)$ & $6349(3)$ & $2755(3)$ & $12829(4)$ & $60(2)$ \\
\hline
\end{tabular}


Table S3. Bond lengths $(\AA)$ and angles $\left({ }^{\circ}\right)$ for $\mathbf{1}$.

\begin{tabular}{|c|c|c|c|}
\hline $\operatorname{Zr}(1)-N(1 \mathbf{A})$ & $2.039(3)$ & $\operatorname{Zr}(1)-N(1)$ & $2.039(3)$ \\
\hline $\operatorname{Zr}(1)-N(2)$ & $2.063(5)$ & $\operatorname{Zr}(1)-S 1(1 A)$ & $2.9331(13)$ \\
\hline $\operatorname{Zr}(1)-S i(1)$ & $2.9331(14)$ & $S i(1)-c(20)$ & $1.920(5)$ \\
\hline$s i(1)-c(10)$ & $1.934(4)$ & $S 1(1)-C(30)$ & $1.944(4)$ \\
\hline$N(1)-C(1)$ & $1.442(6)$ & $N(1)-C(2)$ & $1.448(7)$ \\
\hline$N(2)-C(3)$ & $1.442(6)$ & $N(2)-C(3 A)$ & $1.442(5)$ \\
\hline $0(1)-c(44)$ & $1.345(9)$ & $0(1)-c(41)$ & $1.348(10)$ \\
\hline$O(1)-L I$ & $1.915(8)$ & $O(2)-c(54)$ & $1.427(6)$ \\
\hline$o(2)-c(51)$ & $1.438(7)$ & $O(2)-L I$ & $1.901(8)$ \\
\hline$L i-O(2 a)$ & $1.901(8)$ & LI-0(1a) & $1.915(8)$ \\
\hline$c(10)-c(11)$ & $1.385(6)$ & $c(10)-c(15)$ & $1.394(6)$ \\
\hline$c(11)-c(12)$ & $1.392(7)$ & $c(12)-c(13)$ & $1.372(8)$ \\
\hline$c(13)-c(14)$ & $1.360(8)$ & $c(14)-c(15)$ & $1.396(7)$ \\
\hline$c(20)-c(21)$ & $1.399(6)$ & $c(20)-c(25)$ & $1.403(6)$ \\
\hline$c(21)-c(22)$ & $1.407(7)$ & $c(22)-c(23)$ & $1.380(8)$ \\
\hline$c(23)-c(24)$ & $1.364(8)$ & $c(24)-c(25)$ & $1.373(7)$ \\
\hline$c(30)-c(31)$ & $1.531(6)$ & $c(30)-c(32)$ & $1.536(6)$ \\
\hline$c(30)-c(33)$ & $1.544(6)$ & $c(41)-c(42)$ & $1.389(14)$ \\
\hline$c(42)-c(43)$ & $1.39(2)$ & $C(43)-C(44)$ & $1.395(13)$ \\
\hline$c(51)-c(52)$ & $1.471(8)$ & $c(52)-C(53)$ & $1.498(8)$ \\
\hline$c(53)-c(54)$ & $1.501(8)$ & & \\
\hline$N(1 A)-\operatorname{Zr}(1)-N(1)$ & $118.2(2)$ & $N(1 A)-\operatorname{Zr}(1)-N(2)$ & $120.88(12)$ \\
\hline$N(1)-\operatorname{Zr}(1)-N(2)$ & $120.88(12)$ & $N(1 A)-\operatorname{Zr}(1)-\operatorname{SI}(1 A)$ & $87.88(11)$ \\
\hline$N(1)-\operatorname{Zr}(1)-S i(1 A)$ & $91.06(11)$ & $N(2)-\operatorname{Zr}(1)-S i(1 A)$ & $91.03(3)$ \\
\hline$N(1 A)-\operatorname{Zr}(1)-S i(1)$ & $91.06(11)$ & $N(1)-\operatorname{Zr}(1)-S i(1)$ & $87.88(11)$ \\
\hline$N(2)-\operatorname{Zr}(1)-S i(1)$ & $91.03(2)$ & $S i(1 A)-\operatorname{Zr}(1)-S i(1)$ & $177.93(5)$ \\
\hline$C(20)-S i(1)-C(10)$ & $102.4(2)$ & $C(20)-S i(1)-c(30)$ & $107.9(2)$ \\
\hline$c(10)-\operatorname{Si}(1)-c(30)$ & $101.8(2)$ & $C(20)-S i(1)-\operatorname{Zr}(1)$ & $107.70(13)$ \\
\hline$c(10)-S I(1)-\operatorname{Zr}(1)$ & $120.99(13)$ & $c(30)-S 1(1)-\operatorname{Zr}(1)$ & $114.82(13)$ \\
\hline$C(1)-N(1)-C(2)$ & $110.5(4)$ & $C(1)-N(1)-\operatorname{Zr}(1)$ & $127.4(3)$ \\
\hline$C(2)-N(1)-\operatorname{Zr}(1)$ & $122.0(3)$ & $C(3)-N(2)-C(3 A)$ & $110.9(5)$ \\
\hline$C(3)-N(2)-\operatorname{Zr}(1)$ & $124.6(3)$ & $C(3 A)-N(2)-\operatorname{Zr}(1)$ & $124.6(3)$ \\
\hline$c(44)-0(1)-c(41)$ & $103.8(7)$ & $c(44)-0(1)-L 1$ & $127.9(6)$ \\
\hline$C(41)-O(1)-L i$ & $128.0(6)$ & $c(54)-0(2)-c(51)$ & $108.6(4)$ \\
\hline$C(54)-0(2)-L i$ & $126.1(3)$ & $C(51)-0(2)-L i$ & $122.9(4)$ \\
\hline$O(2)-L i-O(2 a)$ & $109.0(7)$ & $O(2)-\mathrm{L} 1-O(1)$ & $106.7(2)$ \\
\hline $0(2 a)-L i-0(1)$ & $112.5(2)$ & $O(2)-L i-O(1 a)$ & $112.5(2)$ \\
\hline$O(2 a)-L i-o(1 a)$ & $106.7(2)$ & $0(1)-L i-0(1 a)$ & $109.4(7)$ \\
\hline$c(11)-c(10)-c(15)$ & $115.9(4)$ & $c(11)-c(10)-S 1(1)$ & $119.6(3)$ \\
\hline$c(15)-c(10)-S i(1)$ & $124.5(4)$ & $c(10)-c(11)-c(12)$ & $122.8(5)$ \\
\hline$c(13)-c(12)-c(11)$ & $119.6(5)$ & $c(14)-C(13)-C(12)$ & $119.4(5)$ \\
\hline$c(13)-c(14)-c(15)$ & $120.8(5)$ & $c(10)-c(15)-c(14)$ & $121.5(5)$ \\
\hline$c(21)-c(20)-c(25)$ & $115.6(4)$ & $c(21)-c(20)-S i(1)$ & $117.7(4)$ \\
\hline$c(25)-c(20)-S i(1)$ & $126.3(3)$ & $c(20)-c(21)-c(22)$ & $121.0(5)$ \\
\hline$c(23)-c(22)-c(21)$ & $120.4(5)$ & $C(24)-C(23)-C(22)$ & $119.6(5)$ \\
\hline$c(23)-c(24)-c(25)$ & $119.9(5)$ & $c(24)-c(25)-c(20)$ & $123.5(5)$ \\
\hline$c(31)-c(30)-c(32)$ & $110.1(3)$ & $c(31)-c(30)-c(33)$ & $106.5(4)$ \\
\hline$c(32)-c(30)-c(33)$ & $107.4(4)$ & $c(31)-c(30)-\operatorname{si}(1)$ & $109.5(3)$ \\
\hline$c(32)-c(30)-S i(1)$ & $114.5(3)$ & $c(33)-c(30)-s i(1)$ & $108.5(3)$ \\
\hline $0(1)-C(41)-C(42)$ & $111.8(10)$ & $c(41)-c(42)-c(43)$ & $102.1(10)$ \\
\hline$c(42)-c(43)-c(44)$ & $104.0(10)$ & $0(1)-c(44)-c(43)$ & $110.4(9)$ \\
\hline$o(2)-C(51)-C(52)$ & $107.3(5)$ & $c(51)-c(52)-c(53)$ & $102.9(5)$ \\
\hline$c(52)-c(53)-c(54)$ & $103.2(5)$ & $o(2)-c(54)-c(53)$ & $106.4(4)$ \\
\hline
\end{tabular}


Table S4. Anisotropic displacement parameters $\left(\AA^{2} \times 10^{3}\right)$ for $\mathbf{1}$. The anisotropic displacement factor exponent takes the form: $-2 \pi^{2}\left(h^{2} a^{* 2} U^{11}+\ldots+2 h k a * b * U^{12}\right)$

\begin{tabular}{|c|c|c|c|c|c|c|}
\hline & 011 & $\mathrm{U} 22$ & U33 & $\mathrm{U} 23$ & U13 & U12 \\
\hline $\operatorname{Zr}(1)$ & $24(1)$ & $26(1)$ & $18(1)$ & 0 & $9(1)$ & 0 \\
\hline si(1) & $27(1)$ & $34(1)$ & $24(1)$ & $0(1)$ & $13(1)$ & $-1(1)$ \\
\hline $\mathrm{N}(1)$ & $36(2)$ & $53(3)$ & $30(2)$ & $14(2)$ & $14(2)$ & $5(2)$ \\
\hline$N(2)$ & $36(3)$ & $29(3)$ & $48(3)$ & 0 & $24(3)$ & 0 \\
\hline$O(1)$ & $83(3)$ & $83(3)$ & $72(3)$ & $-32(2)$ & $47(2)$ & $-33(2)$ \\
\hline$O(2)$ & $39(2)$ & $61(2)$ & $61(2)$ & $11(2)$ & $19(2)$ & $-4(2)$ \\
\hline LI & $46(7)$ & $57(8)$ & $57(7)$ & 0 & $27(6)$ & 0 \\
\hline$c(1)$ & $53(3)$ & $85(4)$ & $34(3)$ & $12(3)$ & $18(2)$ & $2(3)$ \\
\hline$C(2)$ & $49(3)$ & $63(3)$ & $56(3)$ & $25(3)$ & $30(3)$ & $18(3)$ \\
\hline$c(3)$ & $56(3)$ & $38(3)$ & $70(3)$ & $-13(3)$ & $39(3)$ & $-5(2)$ \\
\hline$c(10)$ & $26(2)$ & $46(3)$ & $23(2)$ & $3(2)$ & $10(2)$ & $5(2)$ \\
\hline$c(11)$ & $44(3)$ & $53(3)$ & $30(3)$ & $-5(2)$ & $18(2)$ & $-3(2)$ \\
\hline$c(12)$ & $56(3)$ & $66(4)$ & $34(3)$ & $-11(3)$ & $17(3)$ & $6(3)$ \\
\hline$c(13)$ & $50(3)$ & $98(5)$ & $33(3)$ & $-11(3)$ & $22(3)$ & $11(3)$ \\
\hline$c(14)$ & $50(3)$ & $104(5)$ & 41(3) & $-3(3)$ & $32(3)$ & $-7(3)$ \\
\hline$c(15)$ & $38(3)$ & $75(4)$ & $32(3)$ & $-3(2)$ & $20(2)$ & $-3(2)$ \\
\hline$c(20)$ & $37(2)$ & $38(3)$ & $38(3)$ & $4(2)$ & $28(2)$ & $4(2)$ \\
\hline$C(21)$ & $54(3)$ & $46(3)$ & $49(3)$ & $8(2)$ & $33(3)$ & $4(2)$ \\
\hline$c(22)$ & $88(4)$ & $45(3)$ & $70(4)$ & $25(3)$ & $56(4)$ & $19(3)$ \\
\hline$C(23)$ & $90(4)$ & $37(3)$ & $84(4)$ & $-11(3)$ & $66(4)$ & $-9(3)$ \\
\hline$C(24)$ & $67(3)$ & $38(3)$ & $64(3)$ & $-12(3)$ & $47(3)$ & $-15(3)$ \\
\hline$c(25)$ & $46(3)$ & $39(3)$ & $44(3)$ & $-4(2)$ & $29(2)$ & $-5(2)$ \\
\hline$c(30)$ & $29(2)$ & $37(2)$ & $23(2)$ & $-4(2)$ & $10(2)$ & $0(2)$ \\
\hline$c(31)$ & $42(3)$ & $47(3)$ & $28(2)$ & $1(2)$ & $14(2)$ & $4(2)$ \\
\hline$c(32)$ & $30(2)$ & $44(3)$ & $39(3)$ & $-4(2)$ & $13(2)$ & $1(2)$ \\
\hline$c(33)$ & $38(3)$ & $35(3)$ & $39(3)$ & $1(2)$ & $15(2)$ & $3(2)$ \\
\hline$c(41)$ & $327(19)$ & $265(17)$ & $260(16)$ & $-177(14)$ & $249(16)$ & $-181(15)$ \\
\hline$c(42)$ & $385(24)$ & $118(9)$ & $104(8)$ & $-27(7)$ & $130(13)$ & $6(12)$ \\
\hline$c(43)$ & $124(9)$ & $219(15)$ & $225(15)$ & $-172(13)$ & $34(9)$ & $-57(9)$ \\
\hline$C(44)$ & $185(11)$ & $178(11)$ & $256(15)$ & $-121(11)$ & $167(11)$ & $-114(10)$ \\
\hline$c(51)$ & $61(4)$ & $97(5)$ & $75(4)$ & $29(4)$ & $20(3)$ & $1(4)$ \\
\hline$C(52)$ & $69(4)$ & $56(4)$ & $78(4)$ & $10(3)$ & $48(3)$ & $10(3)$ \\
\hline$c(53)$ & $60(4)$ & $61(4)$ & $77(4)$ & $-8(3)$ & $40(3)$ & $-8(3)$ \\
\hline$C(54)$ & $49(3)$ & $75(4)$ & $53(3)$ & $-2(3)$ & $23(3)$ & $-9(3)$ \\
\hline
\end{tabular}


Table S5. Hydrogen coordinates $\left(\times 10^{4}\right)$ and isotropic displacement parameters $\left(\AA^{2} \times 10^{3}\right)$ for 2 .

\begin{tabular}{|c|c|c|c|c|}
\hline & $\mathbf{x}$ & $y$ & $\mathbf{z}$ & $\mathrm{U}(\mathrm{eq})$ \\
\hline$H(1 A)$ & $5409(3)$ & $3222(4)$ & $9412(3)$ & 80 \\
\hline$H(1 B)$ & $6077(3)$ & $2642(4)$ & $9894(3)$ & 80 \\
\hline$H(1 C)$ & $5272(3)$ & $2367(4)$ & $9627(3)$ & 80 \\
\hline$H(2 A)$ & $5603(3)$ & $1474(3)$ & $8117(3)$ & 80 \\
\hline$H(2 B)$ & $5389(3)$ & $1315(3)$ & $8848(3)$ & 80 \\
\hline$H(2 C)$ & $6194(3)$ & $1590(3)$ & $9116(3)$ & 80 \\
\hline$H(3 A)$ & $5352(3)$ & $4353(3)$ & $8759(4)$ & 80 \\
\hline$H(3 B)$ & $4801(3)$ & $5008(3)$ & $8163(4)$ & 80 \\
\hline$H(3 C)$ & $5623(3)$ & $5008(3)$ & $8355(4)$ & 80 \\
\hline$H(11 A)$ & $4181(2)$ & $4254(3)$ & $8779(3)$ & 80 \\
\hline$H(12 A)$ & $4080(3)$ & $4707(3)$ & $9943(3)$ & 80 \\
\hline$H(13 A)$ & $3443(3)$ & $3998(4)$ & $10458(3)$ & 80 \\
\hline$H(14 A)$ & $2933(3)$ & $2837(4)$ & $9821(3)$ & 80 \\
\hline$H(15 A)$ & $3007(2)$ & $2394(3)$ & $8632(3)$ & 80 \\
\hline$H(21 A)$ & $4064(3)$ & $1607(3)$ & $8547(3)$ & 80 \\
\hline$H(22 A)$ & $3778(3)$ & $296(3)$ & $8352(4)$ & 80 \\
\hline$H(23 A)$ & $2847(3)$ & $-156(3)$ & $7035(4)$ & 80 \\
\hline$H(24 A)$ & $2193(3)$ & $696(3)$ & $5913(4)$ & 80 \\
\hline$H(25 A)$ & $2460(3)$ & $1984(3)$ & $6099(3)$ & 80 \\
\hline$H(31 A)$ & $3301(2)$ & $3583(3)$ & $5836(3)$ & 80 \\
\hline$H(31 B)$ & $2473(2)$ & $3863(3)$ & $5346(3)$ & 80 \\
\hline $\mathrm{H}(31 \mathrm{C})$ & $2660(2)$ & $2975(3)$ & $5577(3)$ & 80 \\
\hline$H(32 A)$ & $2136(2)$ & $3589(3)$ & $7117(3)$ & 80 \\
\hline$H(32 B)$ & $1944(2)$ & $2979(3)$ & $6362(3)$ & 80 \\
\hline$H(32 C)$ & $1758(2)$ & $3867(3)$ & $6131(3)$ & 80 \\
\hline$H(33 A)$ & $3090(2)$ & $4593(2)$ & $7436(3)$ & 80 \\
\hline$H(33 B)$ & $2686(2)$ & $4808(2)$ & $6429(3)$ & 80 \\
\hline$H(33 C)$ & $3530(2)$ & $4587(2)$ & $6920(3)$ & 80 \\
\hline$H(41 A)$ & $5125(8)$ & $1027(8)$ & $11127(9)$ & 80 \\
\hline$H(41 B)$ & $4492(8)$ & $1598(8)$ & $10489(9)$ & 80 \\
\hline $\mathrm{H}(42 \mathrm{~A})$ & $4475(9)$ & $39(7)$ & $10584(7)$ & 80 \\
\hline$H(42 B)$ & $4043(9)$ & $594(7)$ & $9757(7)$ & 80 \\
\hline$H(43 A)$ & $3430(6)$ & $3(8)$ & $10509(9)$ & 80 \\
\hline$H(43 B)$ & $3200(6)$ & $835(8)$ & $10060(9)$ & 80 \\
\hline$H(44 A)$ & $3491(6)$ & $1185(7)$ & $11333(8)$ & 80 \\
\hline$H(44 B)$ & $4018(6)$ & $465(7)$ & $11770(8)$ & 80 \\
\hline$H(51 A)$ & $4991(4)$ & $3492(4)$ & $11621(4)$ & 80 \\
\hline$H(51 B)$ & $5042(4)$ & $2864(4)$ & $10993(4)$ & 80 \\
\hline$H(52 A)$ & $5812(3)$ & $4074(3)$ & $11395(4)$ & 80 \\
\hline $\mathrm{H}(52 \mathrm{~B})$ & $6125(3)$ & $3277(3)$ & $11253(4)$ & 80 \\
\hline$H(53 A)$ & $6418(3)$ & $3942(3)$ & $12861(4)$ & 80 \\
\hline$H(53 B)$ & $6993(3)$ & $3541(3)$ & $12641(4)$ & 80 \\
\hline$H(54 A)$ & $6667(3)$ & $2354(3)$ & $12799(4)$ & 80 \\
\hline$H(54 B)$ & $6444(3)$ & $2786(3)$ & $13432(4)$ & 80 \\
\hline
\end{tabular}


Table S6. Crystal data and structure refinement for 2 .

\begin{tabular}{|c|c|c|}
\hline Empirical formula & \multicolumn{2}{|c|}{ C54 H88 Hf1 Li1 N3 O4 Si2 } \\
\hline Formula weight & \multicolumn{2}{|l|}{1084.88} \\
\hline Temperature & \multicolumn{2}{|l|}{$173(2) \mathrm{K}$} \\
\hline Wavelength & \multicolumn{2}{|l|}{$0.71073 \AA$} \\
\hline Crystal system & \multicolumn{2}{|l|}{ Monoclinic } \\
\hline Space group & \multicolumn{2}{|l|}{$\mathrm{C} 2 / \mathrm{c}$} \\
\hline \multirow[t]{3}{*}{ Unit cell dimensions } & $\mathrm{a}=21.139(4) \AA$ & $\alpha=90^{\circ}$ \\
\hline & $\mathrm{b}=17.466(3) \AA$ & $\beta=119.699(3)^{\circ}$ \\
\hline & $c=17.762(3) \AA$ & $\gamma=90^{\circ}$ \\
\hline Volume & \multicolumn{2}{|l|}{$5696.8(17) \AA^{3}$} \\
\hline $\mathrm{Z}$ & \multicolumn{2}{|l|}{4} \\
\hline Density (calculated) & \multicolumn{2}{|l|}{$1.265 \mathrm{~g} / \mathrm{cm}^{3}$} \\
\hline Absorption coefficient & \multicolumn{2}{|l|}{$1.916 \mathrm{~mm}^{-1}$} \\
\hline $\mathrm{F}(000)$ & \multicolumn{2}{|l|}{2272} \\
\hline Crystal size & \multicolumn{2}{|c|}{$0.35 \times 0.25 \times 0.25 \mathrm{~mm}^{3}$} \\
\hline Theta range for data collection & \multicolumn{2}{|l|}{1.61 to $28.30^{\circ}$} \\
\hline Index ranges & \multicolumn{2}{|c|}{$-28 \leq \mathrm{h} \leq 27,-23 \leq \mathrm{k} \leq 23,-23 \leq 1 \leq 23$} \\
\hline Reflections collected & \multicolumn{2}{|l|}{29219} \\
\hline Independent reflections & \multicolumn{2}{|c|}{$6865[\mathrm{R}(\mathrm{int})=0.0642]$} \\
\hline Completeness to theta $=28.30^{\circ}$ & \multicolumn{2}{|l|}{$96.8 \%$} \\
\hline Absorption correction & \multicolumn{2}{|c|}{ Semi-empirical from equivalents } \\
\hline Max. and min. transmission & \multicolumn{2}{|c|}{0.6459 and 0.5536} \\
\hline Refinement method & \multicolumn{2}{|c|}{ Full-matrix least-squares on $\mathrm{F}^{2}$} \\
\hline Data / restraints / parameters & \multicolumn{2}{|l|}{$6865 / 0 / 301$} \\
\hline Goodness-of-fit on $\mathrm{F}^{2}$ & \multicolumn{2}{|l|}{0.907} \\
\hline Final $R$ indices $[I>2 \operatorname{sigma}(I)]$ & \multicolumn{2}{|c|}{$\mathrm{R} 1=0.0401, \mathrm{wR} 2=0.1096$} \\
\hline $\mathrm{R}$ indices (all data) & \multicolumn{2}{|c|}{$\mathrm{R} 1=0.0509, \mathrm{wR} 2=0.1209$} \\
\hline Largest diff. peak and hole & \multicolumn{2}{|c|}{1.311 and -0.572 e. $\AA^{-3}$} \\
\hline
\end{tabular}


Table S7. Atomic coordinates $\left(\times 10^{4}\right)$ and equivalent isotropic displacement parameters $\left(\AA^{2} \times\right.$ $10^{3}$ ) for 2 . $U(e q)$ is defined as one third of the trace of the orthogonalized $\mathrm{U}^{\mathrm{ij}}$ tensor.

\begin{tabular}{|c|c|c|c|c|}
\hline & $\mathrm{X}$ & $\mathrm{y}$ & $\mathrm{z}$ & $\mathrm{U}(\mathrm{eq})$ \\
\hline $\mathrm{C}(1)$ & $4794(3)$ & $5323(3)$ & $6746(3)$ & $40(1)$ \\
\hline$C(2)$ & $4442(3)$ & $7308(3)$ & $5550(3)$ & $45(1)$ \\
\hline $\mathrm{C}(3)$ & $4314(3)$ & $8364(3)$ & $6320(4)$ & $44(1)$ \\
\hline $\mathrm{C}(4)$ & $7134(2)$ & $6363(2)$ & $8358(2)$ & $23(1)$ \\
\hline$C(5)$ & $6930(2)$ & $5517(2)$ & $8118(3)$ & $29(1)$ \\
\hline$C(6)$ & $7173(2)$ & $6498(3)$ & $9231(3)$ & $31(1)$ \\
\hline$C(7)$ & $7884(2)$ & $6491(3)$ & $8442(3)$ & $31(1)$ \\
\hline $\mathrm{C}(8)$ & $6402(2)$ & $6715(2)$ & $6448(2)$ & $25(1)$ \\
\hline $\mathrm{C}(9)$ & $6076(2)$ & $6031(3)$ & $6012(3)$ & $35(1)$ \\
\hline$C(10)$ & $6144(3)$ & $5761(3)$ & $5324(3)$ & $43(1)$ \\
\hline $\mathrm{C}(11)$ & $6511(3)$ & $6186(4)$ & $5009(3)$ & $49(1)$ \\
\hline$C(12)$ & $6820(3)$ & $6864(4)$ & $5403(4)$ & $52(2)$ \\
\hline$C(13)$ & $6762(2)$ & $7133(3)$ & $6100(3)$ & $36(1)$ \\
\hline$C(14)$ & $6700(2)$ & $8039(2)$ & $7652(3)$ & $26(1)$ \\
\hline$C(15)$ & $6317(3)$ & $8574(3)$ & $6991(3)$ & $38(1)$ \\
\hline$C(16)$ & $6478(4)$ & $9359(3)$ & $7099(4)$ & $53(1)$ \\
\hline$C(17)$ & $7037(3)$ & $9631(3)$ & $7878(4)$ & $48(1)$ \\
\hline $\mathrm{C}(18)$ & $7427(3)$ & $9125(3)$ & $8547(4)$ & $42(1)$ \\
\hline C(19) & $7262(2)$ & $8349(2)$ & $8434(3)$ & $31(1)$ \\
\hline $\mathrm{C}(20)$ & $8641(3)$ & $7754(3)$ & $7174(4)$ & $45(1)$ \\
\hline $\mathrm{C}(21)$ & $8510(3)$ & $8525(3)$ & $7459(4)$ & $50(1)$ \\
\hline$C(22)$ & $9051(3)$ & $8545(3)$ & $8397(4)$ & $50(1)$ \\
\hline$C(23)$ & $9679(3)$ & $8141(4)$ & $8439(5)$ & $63(2)$ \\
\hline$C(24)$ & $8870(7)$ & $5866(8)$ & $6348(10)$ & $167(7)$ \\
\hline$C(25)$ & $8588(7)$ & $5481(8)$ & $5554(9)$ & $144(5)$ \\
\hline$C(26)$ & $9151(11)$ & $5522(7)$ & $5351(8)$ & $175(8)$ \\
\hline$C(27)$ & $9559(10)$ & $6208(8)$ & $5791(9)$ & $179(8)$ \\
\hline $\mathrm{Hf}(1)$ & 5000 & $6971(1)$ & 7500 & $22(1)$ \\
\hline $\operatorname{Li}(1)$ & 10000 & $6931(6)$ & 7500 & $42(3)$ \\
\hline $\mathrm{N}(1)$ & 5000 & $5792(3)$ & 7500 & $28(1)$ \\
\hline $\mathrm{N}(2)$ & $4541(2)$ & $7569(2)$ & $6369(2)$ & $30(1)$ \\
\hline $\mathrm{O}(1)$ & $9395(2)$ & $7584(2)$ & $7752(2)$ & $45(1)$ \\
\hline $\mathrm{O}(2)$ & $9452(2)$ & $6319(2)$ & $6497(3)$ & $61(1)$ \\
\hline $\operatorname{Si}(1)$ & $6364(1)$ & $7001(1)$ & $7473(1)$ & $20(1)$ \\
\hline
\end{tabular}


Table S8. Bond lengths $(\AA)$ and angles $\left({ }^{\circ}\right)$ for 2 .

\begin{tabular}{|c|c|c|c|}
\hline $\mathrm{C}(1)-\mathrm{N}(1)$ & $1.441(5)$ & $\mathrm{C}(20)-\mathrm{O}(1)$ & $1.436(6)$ \\
\hline $\mathrm{C}(2)-\mathrm{N}(2)$ & $1.439(6)$ & $\mathrm{C}(20)-\mathrm{C}(21)$ & $1.511(8)$ \\
\hline $\mathrm{C}(3)-\mathrm{N}(2)$ & $1.457(7)$ & $\mathrm{C}(21)-\mathrm{C}(22)$ & $1.483(8)$ \\
\hline$C(4)-C(7)$ & $1.533(5)$ & $\mathrm{C}(22)-\mathrm{C}(23)$ & $1.474(8)$ \\
\hline$C(4)-C(6)$ & $1.529(6)$ & $\mathrm{C}(23)-\mathrm{O}(1)$ & $1.439(7)$ \\
\hline$C(4)-C(5)$ & $1.539(5)$ & $\mathrm{C}(24)-\mathrm{C}(25)$ & $1.401(14)$ \\
\hline C(4)-Si(1) & $1.957(4)$ & $\mathrm{C}(24)-\mathrm{O}(2)$ & $1.373(9)$ \\
\hline$C(8)-C(13)$ & $1.400(6)$ & $C(25)-C(26)$ & $1.408(18)$ \\
\hline$C(8)-C(9)$ & $1.404(6)$ & $C(26)-C(27)$ & $1.459(15)$ \\
\hline $\mathrm{C}(8)-\mathrm{Si}(1)$ & $1.928(4)$ & $\mathrm{C}(27)-\mathrm{O}(2)$ & $1.394(11)$ \\
\hline C(9)-C(10) & $1.382(6)$ & $\mathrm{Hf}(1)-\mathrm{N}(2) \# 1$ & $2.033(3)$ \\
\hline $\mathrm{C}(10)-\mathrm{C}(11)$ & $1.377(8)$ & $\mathrm{Hf}(1)-\mathrm{N}(2)$ & $2.033(3)$ \\
\hline $\mathrm{C}(11)-\mathrm{C}(12)$ & $1.366(8)$ & $\mathrm{Hf}(1)-\mathrm{N}(1)$ & $2.060(5)$ \\
\hline$C(12)-C(13)$ & $1.385(7)$ & $\mathrm{Hf}(1)-\mathrm{Si}(1) \# 1$ & $2.9069(11)$ \\
\hline$C(14)-C(15)$ & $1.401(6)$ & $\operatorname{Hf}(1)-\operatorname{Si}(1)$ & $2.9069(11)$ \\
\hline $\mathrm{C}(14)-\mathrm{C}(19)$ & $1.413(6)$ & $\mathrm{Li}(1)-\mathrm{O}(2)$ & $1.901(7)$ \\
\hline C(14)-Si(1) & $1.917(4)$ & $\mathrm{Li}(1)-\mathrm{O}(2) \# 2$ & $1.901(7)$ \\
\hline$C(15)-C(16)$ & $1.403(7)$ & $\mathrm{Li}(1)-\mathrm{O}(1)$ & $1.925(7)$ \\
\hline$C(16)-C(17)$ & $1.383(9)$ & $\mathrm{Li}(1)-\mathrm{O}(1) \# 2$ & $1.925(7)$ \\
\hline $\mathrm{C}(17)-\mathrm{C}(18)$ & $1.379(8)$ & N(1)-C(1)\#1 & $1.441(5)$ \\
\hline $\mathrm{C}(18)-\mathrm{C}(19)$ & $1.390(6)$ & & \\
\hline$C(7)-C(4)-C(6)$ & $110.2(3)$ & $\mathrm{C}(18)-\mathrm{C}(19)-\mathrm{C}(14)$ & $122.9(4)$ \\
\hline$C(7)-C(4)-C(5)$ & $107.5(3)$ & $\mathrm{O}(1)-\mathrm{C}(20)-\mathrm{C}(21)$ & $105.6(4)$ \\
\hline$C(6)-C(4)-C(5)$ & $107.2(3)$ & $\mathrm{C}(22)-\mathrm{C}(21)-\mathrm{C}(20)$ & $103.3(4)$ \\
\hline $\mathrm{C}(7)-\mathrm{C}(4)-\mathrm{Si}(1)$ & $113.8(3)$ & $\mathrm{C}(23)-\mathrm{C}(22)-\mathrm{C}(21)$ & $102.7(5)$ \\
\hline $\mathrm{C}(6)-\mathrm{C}(4)-\mathrm{Si}(1)$ & $109.4(3)$ & $\mathrm{O}(1)-\mathrm{C}(23)-\mathrm{C}(22)$ & $107.1(5)$ \\
\hline C(5)-C(4)-Si(1) & $108.5(2)$ & $\mathrm{C}(25)-\mathrm{C}(24)-\mathrm{O}(2)$ & $111.4(10)$ \\
\hline $\mathrm{C}(13)-\mathrm{C}(8)-\mathrm{C}(9)$ & $115.1(4)$ & $\mathrm{C}(24)-\mathrm{C}(25)-\mathrm{C}(26)$ & $104.0(9)$ \\
\hline $\mathrm{C}(13)-\mathrm{C}(8)-\mathrm{Si}(1)$ & $124.7(3)$ & $\mathrm{C}(25)-\mathrm{C}(26)-\mathrm{C}(27)$ & $104.8(11)$ \\
\hline $\mathrm{C}(9)-\mathrm{C}(8)-\mathrm{Si}(1)$ & $120.2(3)$ & $\mathrm{O}(2)-\mathrm{C}(27)-\mathrm{C}(26)$ & $106.3(9)$ \\
\hline $\mathrm{C}(10)-\mathrm{C}(9)-\mathrm{C}(8)$ & $122.7(4)$ & $\mathrm{N}(2) \# 1-\mathrm{Hf}(1)-\mathrm{N}(2)$ & $118.2(2)$ \\
\hline$C(9)-C(10)-C(11)$ & $120.2(5)$ & $\mathrm{N}(2) \# 1-\mathrm{Hf}(1)-\mathrm{N}(1)$ & $120.91(11)$ \\
\hline$C(12)-C(11)-C(10)$ & $118.7(5)$ & $\mathrm{N}(2)-\mathrm{Hf}(1)-\mathrm{N}(1)$ & $120.91(11)$ \\
\hline $\mathrm{C}(11)-\mathrm{C}(12)-\mathrm{C}(13)$ & $121.4(5)$ & $\mathrm{N}(2) \# 1-\mathrm{Hf}(1)-\mathrm{Si}(1) \# 1$ & $88.05(10)$ \\
\hline $\mathrm{C}(12)-\mathrm{C}(13)-\mathrm{C}(8)$ & $121.7(5)$ & N(2)-Hf(1)-Si(1)\#1 & $90.89(10)$ \\
\hline $\mathrm{C}(15)-\mathrm{C}(14)-\mathrm{C}(19)$ & $115.1(4)$ & $\mathrm{N}(1)-\mathrm{Hf}(1)-\mathrm{Si}(1) \# 1$ & $91.02(2)$ \\
\hline $\mathrm{C}(15)-\mathrm{C}(14)-\mathrm{Si}(1)$ & $118.6(3)$ & N(2)\#1-Hf(1)-Si(1) & $90.89(10)$ \\
\hline C(19)-C(14)-Si(1) & $125.9(3)$ & N(2)-Hf(1)-Si(1) & $88.05(10)$ \\
\hline$C(16)-C(15)-C(14)$ & $122.4(5)$ & $\mathrm{N}(1)-\mathrm{Hf}(1)-\mathrm{Si}(1)$ & $91.024(19)$ \\
\hline$C(17)-C(16)-C(15)$ & $120.1(5)$ & $\mathrm{Si}(1) \# 1-\mathrm{Hf}(1)-\mathrm{Si}(1)$ & $177.95(4)$ \\
\hline $\mathrm{C}(18)-\mathrm{C}(17)-\mathrm{C}(16)$ & $119.5(5)$ & $\mathrm{O}(2)-\mathrm{Li}(1)-\mathrm{O}(2) \# 2$ & $111.6(6)$ \\
\hline $\mathrm{C}(17)-\mathrm{C}(18)-\mathrm{C}(19)$ & $120.0(5)$ & $\mathrm{O}(2)-\mathrm{Li}(1)-\mathrm{O}(1)$ & $112.57(17)$ \\
\hline
\end{tabular}




$\begin{array}{llll}\mathrm{O}(2) \# 2-\mathrm{Li}(1)-\mathrm{O}(1) & 106.38(18) & \mathrm{C}(20)-\mathrm{O}(1)-\mathrm{Li}(1) & 125.9(3) \\ \mathrm{O}(2)-\mathrm{Li}(1)-\mathrm{O}(1) \# 2 & 106.38(18) & \mathrm{C}(23)-\mathrm{O}(1)-\mathrm{Li}(1) & 123.4(4) \\ \mathrm{O}(2) \# 2-\mathrm{Li}(1)-\mathrm{O}(1) \# 2 & 112.57(17) & \mathrm{C}(27)-\mathrm{O}(2)-\mathrm{C}(24) & 106.0(8) \\ \mathrm{O}(1)-\mathrm{Li}(1)-\mathrm{O}(1) \# 2 & 107.4(6) & \mathrm{C}(27)-\mathrm{O}(2)-\mathrm{Li}(1) & 128.7(6) \\ \mathrm{C}(1)-\mathrm{N}(1)-\mathrm{C}(1) \# 1 & 110.7(5) & \mathrm{C}(24)-\mathrm{O}(2)-\mathrm{Li}(1) & 125.2(6) \\ \mathrm{C}(1)-\mathrm{N}(1)-\mathrm{Hf}(1) & 124.6(3) & \mathrm{C}(14)-\mathrm{Si}(1)-\mathrm{C}(8) & 102.44(18) \\ \mathrm{C}(1) \# 1-\mathrm{N}(1)-\mathrm{Hf}(1) & 124.6(3) & \mathrm{C}(14)-\mathrm{Si}(1)-\mathrm{C}(4) & 108.22(17) \\ \mathrm{C}(2)-\mathrm{N}(2)-\mathrm{C}(3) & 111.3(4) & \mathrm{C}(8)-\mathrm{Si}(1)-\mathrm{C}(4) & 101.78(16) \\ \mathrm{C}(2)-\mathrm{N}(2)-\mathrm{Hf}(1) & 126.5(3) & \mathrm{C}(14)-\mathrm{Si}(1)-\mathrm{Hf}(1) & 107.71(12) \\ \mathrm{C}(3)-\mathrm{N}(2)-\mathrm{Hf}(1) & 122.0(3) & \mathrm{C}(8)-\mathrm{Si}(1)-\mathrm{Hf}(1) & 121.12(12) \\ \mathrm{C}(20)-\mathrm{O}(1)-\mathrm{C}(23) & 108.4(4) & \mathrm{C}(4)-\mathrm{Si}(1)-\mathrm{Hf}(1) & 114.43(12)\end{array}$

Symmetry transformations used to generate equivalent atoms:

$\# 1-\mathrm{x}+1, \mathrm{y},-\mathrm{z}+3 / 2 \quad \# 2-\mathrm{x}+2, \mathrm{y},-\mathrm{z}+3 / 2$ 
Table S9. Anisotropic displacement parameters $\left(\AA^{2} \times 10^{3}\right)$ for 2 . The anisotropic displacement factor exponent takes the form: $-2 \pi^{2}\left(h^{2} a^{* 2} U^{11}+\ldots+2 h k a * b * U^{12}\right)$

\begin{tabular}{|c|c|c|c|c|c|c|}
\hline & $\mathrm{U}^{11}$ & $\mathrm{U}^{22}$ & $\mathrm{U}^{33}$ & $\mathrm{U}^{23}$ & $\mathrm{U}^{13}$ & $\mathrm{U}^{12}$ \\
\hline$C(1)$ & $43(2)$ & $32(2)$ & $53(3)$ & $-11(2)$ & $30(2)$ & $-2(2)$ \\
\hline $\mathrm{C}(2)$ & $43(2)$ & $64(3)$ & $26(2)$ & $10(2)$ & $15(2)$ & $5(2)$ \\
\hline$C(3)$ & $42(2)$ & $48(3)$ & $50(3)$ & $26(2)$ & $27(2)$ & $17(2)$ \\
\hline$C(4)$ & $22(2)$ & $23(2)$ & $21(2)$ & $-1(1)$ & $9(1)$ & $1(1)$ \\
\hline $\mathrm{C}(5)$ & $31(2)$ & $21(2)$ & $32(2)$ & $1(2)$ & $13(2)$ & $3(1)$ \\
\hline$C(6)$ & $36(2)$ & $33(2)$ & $20(2)$ & $2(2)$ & $11(2)$ & $3(2)$ \\
\hline$C(7)$ & $22(2)$ & $35(2)$ & $33(2)$ & $-4(2)$ & $11(2)$ & $0(2)$ \\
\hline $\mathrm{C}(8)$ & $21(2)$ & $36(2)$ & $18(2)$ & $3(2)$ & $10(1)$ & $4(2)$ \\
\hline C(9) & $37(2)$ & $42(2)$ & $27(2)$ & $-3(2)$ & $17(2)$ & $0(2)$ \\
\hline$C(10)$ & $44(2)$ & $50(3)$ & $25(2)$ & $-10(2)$ & $9(2)$ & $3(2)$ \\
\hline$C(11)$ & $42(2)$ & $87(4)$ & $23(2)$ & $-9(2)$ & $19(2)$ & $9(3)$ \\
\hline$C(12)$ & $41(3)$ & $94(5)$ & $32(3)$ & $-5(3)$ & $25(2)$ & $-10(3)$ \\
\hline$C(13)$ & $29(2)$ & $54(3)$ & $28(2)$ & $-4(2)$ & $17(2)$ & $-6(2)$ \\
\hline$C(14)$ & $30(2)$ & $26(2)$ & $32(2)$ & $1(2)$ & $23(2)$ & $-1(1)$ \\
\hline$C(15)$ & $43(2)$ & $35(2)$ & $41(3)$ & $9(2)$ & $24(2)$ & $8(2)$ \\
\hline$C(16)$ & $80(4)$ & $32(2)$ & $67(4)$ & $21(2)$ & $51(3)$ & $14(2)$ \\
\hline$C(17)$ & $74(3)$ & $27(2)$ & $71(4)$ & $-5(2)$ & $58(3)$ & $-10(2)$ \\
\hline $\mathrm{C}(18)$ & $55(3)$ & $34(2)$ & $55(3)$ & $-12(2)$ & $40(3)$ & $-13(2)$ \\
\hline$C(19)$ & $35(2)$ & $26(2)$ & $38(2)$ & $-4(2)$ & $23(2)$ & $-6(2)$ \\
\hline$C(20)$ & $36(2)$ & $49(3)$ & $48(3)$ & $3(2)$ & $18(2)$ & $5(2)$ \\
\hline$C(21)$ & $52(3)$ & $44(3)$ & $63(4)$ & $6(3)$ & $36(3)$ & $8(2)$ \\
\hline$C(22)$ & $62(3)$ & $42(3)$ & $67(4)$ & $-12(2)$ & $47(3)$ & $-11(2)$ \\
\hline$C(23)$ & $45(3)$ & $64(4)$ & $66(4)$ & $-19(3)$ & $18(3)$ & $-8(3)$ \\
\hline$C(24)$ & $160(10)$ & $168(11)$ & $232(15)$ & $-144(11)$ & $143(11)$ & $-129(10)$ \\
\hline $\mathrm{C}(25)$ & $115(8)$ & $147(11)$ & $128(10)$ & $-78(9)$ & $29(8)$ & $-48(8)$ \\
\hline$C(26)$ & $370(20)$ & $92(8)$ & $99(8)$ & $-47(6)$ & $143(13)$ & $-66(12)$ \\
\hline $\mathrm{C}(27)$ & $310(20)$ & $152(11)$ & 171(13) & $-99(10)$ & $196(15)$ & $-117(13)$ \\
\hline $\mathrm{Hf}(1)$ & $24(1)$ & $23(1)$ & 19(1) & 0 & $10(1)$ & 0 \\
\hline $\operatorname{Li}(1)$ & $39(6)$ & $36(6)$ & $45(7)$ & 0 & $16(5)$ & 0 \\
\hline $\mathrm{N}(1)$ & $29(2)$ & $19(2)$ & $37(3)$ & 0 & $18(2)$ & 0 \\
\hline $\mathrm{N}(2)$ & $24(2)$ & $42(2)$ & $21(2)$ & $11(1)$ & $10(1)$ & $5(1)$ \\
\hline $\mathrm{O}(1)$ & $30(2)$ & $45(2)$ & $53(2)$ & $-11(2)$ & $16(2)$ & $1(1)$ \\
\hline $\mathrm{O}(2)$ & $68(3)$ & $63(3)$ & $60(3)$ & $-26(2)$ & $37(2)$ & $-25(2)$ \\
\hline $\operatorname{Si}(1)$ & $20(1)$ & $23(1)$ & $18(1)$ & $0(1)$ & $10(1)$ & $0(1)$ \\
\hline
\end{tabular}


Table S10. Hydrogen coordinates $\left(\times 10^{4}\right)$ and isotropic displacement parameters $\left(\AA^{2} \times 10^{3}\right)$ for 2.

\begin{tabular}{|c|c|c|c|c|}
\hline & $\mathrm{x}$ & $\mathrm{y}$ & z & $\mathrm{U}(\mathrm{eq})$ \\
\hline $\mathrm{H}(1 \mathrm{~A})$ & 5217 & 5030 & 6822 & 59 \\
\hline $\mathrm{H}(1 \mathrm{~B})$ & 4618 & 5650 & 6233 & 59 \\
\hline $\mathrm{H}(1 \mathrm{C})$ & 4406 & 4970 & 6670 & 59 \\
\hline $\mathrm{H}(2 \mathrm{~A})$ & 3921 & 7283 & 5127 & 67 \\
\hline $\mathrm{H}(2 \mathrm{~B})$ & 4658 & 6798 & 5621 & 67 \\
\hline $\mathrm{H}(2 \mathrm{C})$ & 4681 & 7664 & 5343 & 67 \\
\hline $\mathrm{H}(3 \mathrm{~A})$ & 4601 & 8688 & 6151 & 67 \\
\hline $\mathrm{H}(3 \mathrm{~B})$ & 4391 & 8527 & 6887 & 67 \\
\hline $\mathrm{H}(3 \mathrm{C})$ & 3796 & 8410 & 5888 & 67 \\
\hline $\mathrm{H}(5 \mathrm{~A})$ & 6944 & 5397 & 7588 & 44 \\
\hline $\mathrm{H}(5 \mathrm{~B})$ & 6439 & 5424 & 8023 & 44 \\
\hline $\mathrm{H}(5 \mathrm{C})$ & 7279 & 5190 & 8592 & 44 \\
\hline $\mathrm{H}(6 \mathrm{~A})$ & 7557 & 6177 & 9674 & 47 \\
\hline $\mathrm{H}(6 \mathrm{~B})$ & 6704 & 6365 & 9185 & 47 \\
\hline $\mathrm{H}(6 \mathrm{C})$ & 7282 & 7038 & 9393 & 47 \\
\hline $\mathrm{H}(7 \mathrm{~A})$ & 8018 & 7032 & 8568 & 47 \\
\hline $\mathrm{H}(7 \mathrm{~B})$ & 7865 & 6349 & 7898 & 47 \\
\hline $\mathrm{H}(7 \mathrm{C})$ & 8246 & 6175 & 8915 & 47 \\
\hline $\mathrm{H}(9)$ & 5798 & 5742 & 6198 & 42 \\
\hline $\mathrm{H}(10)$ & 5937 & 5282 & 5069 & 52 \\
\hline H(11) & 6548 & 6010 & 4526 & 59 \\
\hline $\mathrm{H}(12)$ & 7079 & 7157 & 5195 & 63 \\
\hline $\mathrm{H}(13)$ & 6972 & 7613 & 6349 & 43 \\
\hline $\mathrm{H}(15)$ & 5934 & 8398 & 6450 & 46 \\
\hline H(16) & 6204 & 9704 & 6637 & 64 \\
\hline $\mathrm{H}(17)$ & 7150 & 10161 & 7951 & 57 \\
\hline $\mathrm{H}(18)$ & 7808 & 9308 & 9086 & 51 \\
\hline H(19) & 7539 & 8011 & 8904 & 38 \\
\hline $\mathrm{H}(20 \mathrm{~A})$ & 8325 & 7359 & 7218 & 54 \\
\hline $\mathrm{H}(20 \mathrm{~B})$ & 8539 & 7776 & 6567 & 54 \\
\hline $\mathrm{H}(21 \mathrm{~A})$ & 8594 & 8946 & 7146 & 60 \\
\hline $\mathrm{H}(21 \mathrm{~B})$ & 8007 & 8564 & 7360 & 60 \\
\hline $\mathrm{H}(22 \mathrm{~A})$ & 9179 & 9078 & 8608 & 60 \\
\hline $\mathrm{H}(22 \mathrm{~B})$ & 8867 & 8277 & 8741 & 60 \\
\hline $\mathrm{H}(23 \mathrm{~A})$ & 10001 & 8507 & 8362 & 75 \\
\hline $\mathrm{H}(23 \mathrm{~B})$ & 9965 & 7885 & 9007 & 75 \\
\hline $\mathrm{H}(24 \mathrm{~A})$ & 9026 & 5487 & 6822 & 200 \\
\hline $\mathrm{H}(24 \mathrm{~B})$ & 8484 & 6188 & 6347 & 200 \\
\hline $\mathrm{H}(25 \mathrm{~A})$ & 8141 & 5735 & 5104 & 173 \\
\hline $\mathrm{H}(25 \mathrm{~B})$ & 8472 & 4942 & 5611 & 173 \\
\hline $\mathrm{H}(26 \mathrm{~A})$ & 8950 & 5564 & 4717 & 210 \\
\hline
\end{tabular}




\begin{tabular}{lrlll}
$\mathrm{H}(26 \mathrm{~B})$ & 9466 & 5062 & 5563 & 210 \\
$\mathrm{H}(27 \mathrm{~A})$ & 10083 & 6139 & 5990 & 215 \\
$\mathrm{H}(27 \mathrm{~B})$ & 9379 & 6655 & 5397 & 215 \\
& & & & \\
\hline
\end{tabular}


Table S11. Crystal data and structure refinement for 3

\begin{tabular}{|c|c|c|}
\hline Empirical formula & \multicolumn{2}{|c|}{ C360 H840 C10 K8 N24 O72 Si48 Zr8 } \\
\hline Formula weight & \multicolumn{2}{|l|}{9049.44} \\
\hline Temperature & \multicolumn{2}{|l|}{$273(2) \mathrm{K}$} \\
\hline Wavelength & \multicolumn{2}{|l|}{$0.71073 \AA$} \\
\hline Crystal system & \multicolumn{2}{|l|}{ Triclinic } \\
\hline Space group & \multicolumn{2}{|l|}{$\mathrm{P}-1$} \\
\hline \multirow[t]{3}{*}{ Unit cell dimensions } & $a=13.077(3) \AA$ & $\alpha=101.06(3)^{\circ}$. \\
\hline & $\mathrm{b}=24.797(5) \AA$ & $\beta=92.00(3)^{\circ}$. \\
\hline & $\mathrm{c}=41.823(8) \AA$ & $\gamma=101.05(3)^{\circ}$. \\
\hline Volume & \multicolumn{2}{|l|}{$13028(5) \AA^{3}$} \\
\hline $\mathrm{Z}$ & \multicolumn{2}{|l|}{1} \\
\hline Density (calculated) & \multicolumn{2}{|l|}{$1.153 \mathrm{Mg} / \mathrm{m}^{3}$} \\
\hline Absorption coefficient & \multicolumn{2}{|l|}{$0.388 \mathrm{~mm}^{-1}$} \\
\hline $\mathrm{F}(000)$ & \multicolumn{2}{|l|}{4888} \\
\hline Crystal size & \multicolumn{2}{|c|}{$0.45 \times 0.40 \times 0.20 \mathrm{~mm}^{3}$} \\
\hline Theta range for data collection & \multicolumn{2}{|l|}{1.07 to $27.05^{\circ}$. } \\
\hline Index ranges & \multicolumn{2}{|c|}{$-16 \leq \mathrm{h} \leq 16,-30 \leq \mathrm{k} \leq 30,-52 \leq 1 \leq 52$} \\
\hline Reflections collected & \multicolumn{2}{|l|}{119093} \\
\hline Independent reflections & \multicolumn{2}{|c|}{$52829[\mathrm{R}(\mathrm{int})=0.1100]$} \\
\hline Completeness to theta $=27.05^{\circ}$ & \multicolumn{2}{|l|}{$92.4 \%$} \\
\hline Max. and min. transmission & \multicolumn{2}{|c|}{0.9265 and 0.8448} \\
\hline Refinement method & \multicolumn{2}{|c|}{ Full-matrix least-squares on $\mathrm{F}^{2}$} \\
\hline Data / restraints / parameters & \multicolumn{2}{|l|}{$52829 / 0 / 2419$} \\
\hline Goodness-of-fit on $\mathrm{F}^{2}$ & \multicolumn{2}{|l|}{1.004} \\
\hline Final R indices $[\mathrm{I}>2 \operatorname{sigma}(\mathrm{I})]$ & \multicolumn{2}{|c|}{$\mathrm{R} 1=0.1096, \mathrm{wR} 2=0.2836$} \\
\hline $\mathrm{R}$ indices (all data) & \multicolumn{2}{|c|}{$\mathrm{R} 1=0.1932, \mathrm{wR} 2=0.3558$} \\
\hline Largest diff. peak and hole & \multicolumn{2}{|c|}{2.536 and -1.124 e. $\AA^{-3}$} \\
\hline
\end{tabular}


Table S12. Atomic coordinates $\left(\times 10^{4}\right)$ and equivalent isotropic displacement parameters $\left(\AA^{2} \times\right.$ $10^{3}$ ) for 3 . $\mathrm{U}(\mathrm{eq})$ is defined as one third of the trace of the orthogonalized Uij tensor.

\begin{tabular}{|c|c|c|c|c|}
\hline & $\mathrm{X}$ & $\mathrm{y}$ & $\mathrm{Z}$ & $\mathrm{U}(\mathrm{eq})$ \\
\hline $\mathrm{C}(1)$ & 5990 & 1338 & 8216 & 62 \\
\hline$C(2)$ & 5075 & 1727 & 7845 & 82 \\
\hline $\mathrm{C}(3)$ & 2637 & 1153 & 8199 & 75 \\
\hline$C(4)$ & 2189 & 1929 & 7986 & 78 \\
\hline$C(5)$ & 5771 & 3132 & 8227 & 81 \\
\hline$C(6)$ & 4148 & 3312 & 8402 & 58 \\
\hline$C(7)$ & 3238 & 557 & 9022 & 47 \\
\hline $\mathrm{C}(8)$ & 5067 & 270 & 8649 & 51 \\
\hline $\mathrm{C}(9)$ & 5267 & 578 & 9390 & 50 \\
\hline$C(10)$ & 7727 & 1336 & 8955 & 56 \\
\hline $\mathrm{C}(11)$ & 7642 & 2533 & 8889 & 77 \\
\hline$C(12)$ & 7442 & 2206 & 9541 & 55 \\
\hline$C(13)$ & 4772 & 2053 & 9433 & 35 \\
\hline$C(14)$ & 3615 & 2101 & 9419 & 36 \\
\hline$C(15)$ & 950 & 1683 & 8949 & 71 \\
\hline$C(16)$ & 1113 & 2825 & 8745 & 67 \\
\hline $\mathrm{C}(17)$ & 1079 & 2737 & 9469 & 61 \\
\hline $\mathrm{C}(18)$ & 5550 & 3671 & 9235 & 51 \\
\hline C(19) & 4134 & 3549 & 9792 & 58 \\
\hline $\mathrm{C}(20)$ & 3420 & 3976 & 9217 & 54 \\
\hline$C(21)$ & 6900 & -45 & 6906 & 57 \\
\hline$C(22)$ & 6219 & 658 & 7252 & 57 \\
\hline $\mathrm{C}(23)$ & 3702 & 80 & 6898 & 62 \\
\hline $\mathrm{C}(24)$ & 3362 & 918 & 6716 & 55 \\
\hline$C(25)$ & 7022 & 1859 & 6672 & 48 \\
\hline$C(26)$ & 5631 & 2022 & 7028 & 46 \\
\hline$C(27)$ & 4988 & -971 & 6232 & 85 \\
\hline $\mathrm{C}(28)$ & 5811 & -1085 & 5550 & 76 \\
\hline$C(29)$ & 7128 & -1216 & 6136 & 71 \\
\hline$C(30)$ & 9077 & -20 & 5734 & 54 \\
\hline $\mathrm{C}(31)$ & 9199 & 1129 & 6173 & 51 \\
\hline $\mathrm{C}(32)$ & 9309 & 138 & 6486 & 67 \\
\hline $\mathrm{C}(33)$ & 6619 & 378 & 5670 & 30 \\
\hline C(34) & 5472 & 387 & 5578 & 38 \\
\hline$C(35)$ & 2556 & -126 & 5908 & 55 \\
\hline$C(36)$ & 2879 & 352 & 5300 & 57 \\
\hline$C(37)$ & 2248 & 1053 & 5904 & 59 \\
\hline $\mathrm{C}(38)$ & 4639 & 1862 & 5430 & 41 \\
\hline$C(39)$ & 6734 & 1978 & 5787 & 42 \\
\hline $\mathrm{C}(40)$ & 4906 & 2285 & 6164 & 40 \\
\hline $\mathrm{C}(41)$ & 6072 & 3944 & 3169 & 51 \\
\hline
\end{tabular}




\begin{tabular}{|c|c|c|c|c|}
\hline$C(42)$ & 5177 & 4390 & 2810 & 70 \\
\hline$C(43)$ & 2652 & 3672 & 3101 & 78 \\
\hline$C(44)$ & 2129 & 4471 & 2952 & 69 \\
\hline$C(45)$ & 5701 & 5756 & 3252 & 67 \\
\hline$C(46)$ & 3994 & 5866 & 3383 & 47 \\
\hline$C(47)$ & 3159 & 3019 & 3947 & 43 \\
\hline $\mathrm{C}(48)$ & 4967 & 2800 & 3542 & 50 \\
\hline$C(49)$ & 5226 & 3024 & 4291 & 48 \\
\hline $\mathrm{C}(50)$ & 7671 & 3892 & 3952 & 52 \\
\hline $\mathrm{C}(51)$ & 7495 & 5048 & 3834 & 54 \\
\hline$C(52)$ & 7286 & 4783 & 4506 & 50 \\
\hline$C(53)$ & 790 & 4175 & 3819 & 57 \\
\hline $\mathrm{C}(54)$ & 1045 & 5388 & 3702 & 57 \\
\hline$C(55)$ & 892 & 5175 & 4390 & 48 \\
\hline$C(56)$ & 5425 & 6108 & 4237 & 45 \\
\hline$C(57)$ & 4004 & 5921 & 4784 & 50 \\
\hline $\mathrm{C}(58)$ & 3326 & 6456 & 4240 & 52 \\
\hline$C(59)$ & 3431 & 4526 & 4344 & 28 \\
\hline$C(60)$ & 4594 & 4482 & 4380 & 29 \\
\hline $\mathrm{C}(61)$ & 5449 & 1706 & 1718 & 131 \\
\hline$C(62)$ & 7125 & 2200 & 1914 & 149 \\
\hline $\mathrm{C}(63)$ & 4266 & 2675 & 2130 & 118 \\
\hline$C(64)$ & 3807 & 3346 & 1868 & 68 \\
\hline$C(65)$ & 6624 & 4127 & 2076 & 80 \\
\hline$C(66)$ & 7973 & 3729 & 1781 & 62 \\
\hline$C(67)$ & 4576 & 1364 & 821 & 68 \\
\hline $\mathrm{C}(68)$ & 5984 & 1475 & 284 & 64 \\
\hline$C(69)$ & 6755 & 1097 & 881 & 62 \\
\hline$C(70)$ & 8849 & 2228 & 526 & 54 \\
\hline $\mathrm{C}(71)$ & 9176 & 2369 & 1260 & 66 \\
\hline$C(72)$ & 9092 & 3396 & 953 & 59 \\
\hline$C(73)$ & 6362 & 2956 & 648 & 43 \\
\hline$C(74)$ & 5228 & 2977 & 644 & 45 \\
\hline$C(75)$ & 2621 & 2847 & 510 & 82 \\
\hline$C(76)$ & 2433 & 2312 & 1100 & 83 \\
\hline$C(77)$ & 2183 & 3507 & 1145 & 74 \\
\hline $\mathrm{C}(78)$ & 4608 & 4480 & 742 & 61 \\
\hline$C(79)$ & 6636 & 4513 & 1115 & 60 \\
\hline$C(80)$ & 4713 & 4668 & 1482 & 53 \\
\hline $\mathrm{C}(81)$ & 7314 & 4326 & 10065 & 63 \\
\hline $\mathrm{C}(82)$ & 7811 & 3825 & 9992 & 68 \\
\hline $\mathrm{C}(83)$ & 9397 & 3533 & 10043 & 58 \\
\hline $\mathrm{C}(84)$ & 10466 & 3706 & 10205 & 59 \\
\hline $\mathrm{C}(85)$ & 12085 & 4369 & 10232 & 61 \\
\hline$C(86)$ & 12662 & 4784 & 10057 & 61 \\
\hline $\mathrm{C}(87)$ & 8673 & 4637 & 8956 & 40 \\
\hline
\end{tabular}




\begin{tabular}{|c|c|c|c|c|}
\hline $\mathrm{C}(88)$ & 9533 & 5069 & 8980 & 48 \\
\hline C(89) & 10492 & 5036 & 9096 & 68 \\
\hline $\mathrm{C}(90)$ & 10593 & 4541 & 9202 & 84 \\
\hline $\mathrm{C}(91)$ & 9697 & 4112 & 9180 & 79 \\
\hline $\mathrm{C}(92)$ & 8757 & 4153 & 9058 & 56 \\
\hline C(93) & 7597 & 4690 & 8805 & 82 \\
\hline C(99) & 1583 & 4116 & 8206 & 62 \\
\hline $\mathrm{C}(118)$ & 1165 & 3871 & 4795 & 67 \\
\hline$C(119)$ & 2043 & 3698 & 4946 & 59 \\
\hline$C(120)$ & 2859 & 2930 & 4965 & 57 \\
\hline $\mathrm{C}(121)$ & 2717 & 2321 & 4862 & 64 \\
\hline$C(122)$ & 1652 & 1456 & 4911 & 76 \\
\hline $\mathrm{C}(123)$ & 764 & 1217 & 5070 & 74 \\
\hline$C(124)$ & -1070 & 1131 & 5102 & 64 \\
\hline$C(125)$ & -1993 & 1222 & 4942 & 65 \\
\hline$C(126)$ & -2802 & 1976 & 4836 & 63 \\
\hline$C(127)$ & -2704 & 2583 & 4915 & 63 \\
\hline $\mathrm{C}(128)$ & -1670 & 3456 & 4862 & 66 \\
\hline$C(129)$ & -701 & 3710 & 4739 & 75 \\
\hline$C(130)$ & -1476 & 2140 & 3956 & 50 \\
\hline$C(131)$ & -649 & 2571 & 3966 & 50 \\
\hline$C(132)$ & 335 & 2531 & 4062 & 55 \\
\hline$C(133)$ & 511 & 2040 & 4145 & 65 \\
\hline$C(134)$ & -292 & 1594 & 4139 & 63 \\
\hline$C(135)$ & -1326 & 1636 & 4035 & 59 \\
\hline$C(136)$ & -2605 & 2183 & 3858 & 105 \\
\hline$C(137)$ & 1424 & 2821 & 5848 & 31 \\
\hline $\mathrm{C}(138)$ & 1391 & 3348 & 5776 & 36 \\
\hline $\mathrm{C}(139)$ & 409 & 3453 & 5684 & 53 \\
\hline$C(140)$ & -505 & 3056 & 5671 & 62 \\
\hline$C(141)$ & -462 & 2535 & 5740 & 52 \\
\hline$C(142)$ & 522 & 2422 & 5835 & 43 \\
\hline$C(143)$ & 2466 & 2694 & 5954 & 57 \\
\hline$C(144)$ & -513 & 1592 & 7250 & 58 \\
\hline$C(145)$ & 532 & 1646 & 7105 & 61 \\
\hline$C(146)$ & 1606 & 2220 & 6781 & 62 \\
\hline $\mathrm{C}(147)$ & 2604 & 2432 & 6987 & 65 \\
\hline $\mathrm{C}(148)$ & 3513 & 3181 & 7409 & 50 \\
\hline$C(149)$ & 3421 & 3754 & 7596 & 51 \\
\hline$C(150)$ & 2485 & 4228 & 7977 & 52 \\
\hline$C(151)$ & -241 & 3933 & 8195 & 123 \\
\hline$C(152)$ & -921 & 3474 & 8173 & 178 \\
\hline$C(153)$ & -1259 & 2497 & 7963 & 76 \\
\hline$C(154)$ & -1431 & 2046 & 7660 & 61 \\
\hline$C(155)$ & 1669 & 3982 & 6836 & 45 \\
\hline$C(156)$ & 1354 & 4558 & 6939 & 39 \\
\hline
\end{tabular}




\begin{tabular}{|c|c|c|c|c|}
\hline C(157) & 585 & 5072 & 7353 & 49 \\
\hline C(158) & 45 & 5030 & 7673 & 51 \\
\hline C(159) & -693 & 5637 & 8088 & 63 \\
\hline$C(160)$ & -1882 & 5463 & 8042 & 58 \\
\hline$C(161)$ & -3171 & 4678 & 7794 & 58 \\
\hline$C(162)$ & -3282 & 4063 & 7632 & 54 \\
\hline C(163) & -2475 & 3493 & 7252 & 50 \\
\hline C(164) & -1727 & 3541 & 6971 & 46 \\
\hline$C(165)$ & -826 & 2944 & 6623 & 41 \\
\hline$C(166)$ & 33 & 3422 & 6578 & 38 \\
\hline$C(167)$ & -2481 & 405 & -42 & 49 \\
\hline C(168) & -1839 & 797 & -207 & 58 \\
\hline C(169) & -126 & 1362 & -189 & 55 \\
\hline$C(170)$ & 915 & 1480 & -16 & 55 \\
\hline $\mathrm{C}(171)$ & 2398 & 1075 & 68 & 55 \\
\hline $\mathrm{C}(172)$ & 2789 & 556 & -25 & 54 \\
\hline$C(173)$ & 1293 & 340 & 992 & 45 \\
\hline C(174) & 491 & -123 & 974 & 44 \\
\hline$C(175)$ & -500 & -121 & 870 & 54 \\
\hline$C(176)$ & -731 & 350 & 768 & 60 \\
\hline $\mathrm{C}(177)$ & 84 & 829 & 786 & 70 \\
\hline $\mathrm{C}(178)$ & 1081 & 816 & 895 & 53 \\
\hline C(179) & 2416 & 350 & 1117 & 90 \\
\hline $\mathrm{C}(228)$ & 9099 & 827 & 1673 & 102 \\
\hline C(229) & 10118 & 1154 & 1777 & 117 \\
\hline$C(230)$ & 11080 & 2050 & 2037 & 127 \\
\hline$C(231)$ & 10900 & 2580 & 2100 & 109 \\
\hline $\mathrm{C}(232)$ & 10017 & 3228 & 2471 & 129 \\
\hline $\mathrm{C}(233)$ & 9513 & 3266 & 2729 & 102 \\
\hline$C(234)$ & 7971 & 2763 & 3057 & 138 \\
\hline$C(235)$ & 6961 & 2410 & 2945 & 155 \\
\hline$C(236)$ & 6078 & 1492 & 2737 & 127 \\
\hline $\mathrm{C}(237)$ & 6220 & 973 & 2626 & 102 \\
\hline $\mathrm{C}(238)$ & 6956 & 431 & 2167 & 106 \\
\hline C(239) & 7460 & 461 & 1876 & 102 \\
\hline$C(240)$ & 9984 & 1465 & 3246 & 52 \\
\hline $\mathrm{C}(241)$ & 8996 & 1041 & 3130 & 57 \\
\hline $\mathrm{C}(247)$ & 11034 & 2282 & 3107 & 50 \\
\hline$C(248)$ & 12042 & 2078 & 3104 & 47 \\
\hline C(249) & 9780 & 415 & 2770 & 81 \\
\hline $\mathrm{C}(250)$ & 9603 & 28 & 2436 & 80 \\
\hline $\mathrm{C}(251)$ & 13026 & 1546 & 2732 & 66 \\
\hline$C(252)$ & 12815 & 1155 & 2401 & 75 \\
\hline$C(253)$ & 10389 & -552 & 2069 & 110 \\
\hline$C(254)$ & 11439 & -528 & 1985 & 137 \\
\hline$C(255)$ & 12981 & 20 & 2009 & 89 \\
\hline
\end{tabular}




\begin{tabular}{|c|c|c|c|c|}
\hline$C(259)$ & 13632 & 564 & 2023 & 86 \\
\hline $\mathrm{K}(1)$ & 10000 & 5000 & 10000 & 43 \\
\hline$K(2)$ & 554 & 3161 & 7414 & 37 \\
\hline $\mathrm{K}(3)$ & 11 & 2479 & 4922 & 35 \\
\hline $\mathrm{K}(4)$ & 8791 & 1708 & 2465 & 34 \\
\hline $\mathrm{K}(5)$ & 0 & 0 & 0 & 37 \\
\hline $\mathrm{N}(1)$ & 5250 & 1692 & 8197 & 45 \\
\hline $\mathrm{N}(2)$ & 2884 & 1753 & 8209 & 45 \\
\hline $\mathrm{N}(3)$ & 4837 & 2906 & 8369 & 46 \\
\hline $\mathrm{N}(4)$ & 6346 & 399 & 6904 & 43 \\
\hline $\mathrm{N}(5)$ & 4107 & 546 & 6735 & 42 \\
\hline $\mathrm{N}(6)$ & 6012 & 1626 & 6773 & 35 \\
\hline $\mathrm{N}(7)$ & 5294 & 4293 & 3154 & 40 \\
\hline $\mathrm{N}(8)$ & 2883 & 4282 & 3147 & 42 \\
\hline N(9) & 4714 & 5489 & 3357 & 35 \\
\hline $\mathrm{N}(10)$ & 6278 & 2275 & 1763 & 72 \\
\hline $\mathrm{N}(11)$ & 4539 & 2982 & 1873 & 62 \\
\hline $\mathrm{N}(12)$ & 6863 & 3653 & 1822 & 45 \\
\hline $\mathrm{O}(1)$ & 7813 & 4744 & 9904 & 48 \\
\hline $\mathrm{O}(2)$ & 8888 & 3981 & 10113 & 52 \\
\hline $\mathrm{O}(3)$ & 11061 & 4164 & 10068 & 52 \\
\hline $\mathrm{O}(16)$ & 187 & 3597 & 4888 & 56 \\
\hline $\mathrm{O}(17)$ & 1977 & 3129 & 4844 & 52 \\
\hline $\mathrm{O}(18)$ & 1836 & 2059 & 4995 & 57 \\
\hline $\mathrm{O}(19)$ & -173 & 1345 & 4960 & 55 \\
\hline $\mathrm{O}(20)$ & -1941 & 1814 & 4990 & 51 \\
\hline $\mathrm{O}(21)$ & -1781 & 2856 & 4793 & 56 \\
\hline $\mathrm{O}(22)$ & -425 & 2056 & 7520 & 50 \\
\hline $\mathrm{O}(23)$ & 732 & 2161 & 6972 & 53 \\
\hline $\mathrm{O}(24)$ & 2592 & 2969 & 7192 & 54 \\
\hline $\mathrm{O}(25)$ & 2539 & 3695 & 7786 & 42 \\
\hline $\mathrm{O}(26)$ & 648 & 3955 & 8006 & 54 \\
\hline $\mathrm{O}(27)$ & -1012 & 3034 & 7868 & 66 \\
\hline $\mathrm{O}(28)$ & 808 & 3510 & 6833 & 36 \\
\hline $\mathrm{O}(29)$ & 929 & 4572 & 7255 & 44 \\
\hline $\mathrm{O}(30)$ & -242 & 5573 & 7769 & 50 \\
\hline $\mathrm{O}(31)$ & -2155 & 4864 & 7933 & 51 \\
\hline $\mathrm{O}(32)$ & -2605 & 4041 & 7372 & 51 \\
\hline $\mathrm{O}(33)$ & -1261 & 3061 & 6936 & 39 \\
\hline $\mathrm{O}(34)$ & -791 & 934 & -53 & 47 \\
\hline $\mathrm{O}(35)$ & 1375 & 981 & -82 & 48 \\
\hline $\mathrm{O}(36)$ & 2144 & 120 & 111 & 41 \\
\hline $\mathrm{O}(101)$ & 8525 & 775 & 1937 & 83 \\
\hline $\mathrm{O}(102)$ & 10060 & 1715 & 1936 & 98 \\
\hline $\mathrm{O}(103)$ & 10262 & 2717 & 2407 & 97 \\
\hline $\mathrm{O}(104)$ & 8496 & 2824 & 2767 & 124 \\
\hline
\end{tabular}




\begin{tabular}{|c|c|c|c|c|}
\hline $\mathrm{O}(105)$ & 7053 & 1872 & 2831 & 102 \\
\hline $\mathrm{O}(106)$ & 6803 & 943 & 2316 & 82 \\
\hline $\mathrm{O}(107)$ & 8971 & 726 & 2803 & 48 \\
\hline $\mathrm{O}(108)$ & 10110 & 1839 & 3029 & 46 \\
\hline $\mathrm{O}(112)$ & 12059 & 1761 & 2782 & 60 \\
\hline $\mathrm{O}(113)$ & 10423 & -242 & 2400 & 95 \\
\hline $\mathrm{O}(114)$ & 11989 & 40 & 1976 & 91 \\
\hline $\mathrm{O}(117)$ & 13696 & 944 & 2341 & 81 \\
\hline $\mathrm{Si}(1)$ & 5198 & 1737 & 9003 & 25 \\
\hline $\operatorname{Si}(2)$ & 3416 & 2510 & 9077 & 26 \\
\hline $\operatorname{Si}(3)$ & 4708 & 769 & 9011 & 32 \\
\hline $\operatorname{Si}(4)$ & 7029 & 1946 & 9095 & 35 \\
\hline $\operatorname{Si}(5)$ & 1612 & 2454 & 9058 & 40 \\
\hline $\operatorname{Si}(6)$ & 4152 & 3442 & 9333 & 36 \\
\hline $\operatorname{Si}(7)$ & 6775 & 202 & 6097 & 27 \\
\hline $\operatorname{Si}(8)$ & 4887 & 841 & 5928 & 27 \\
\hline $\operatorname{Si}(9)$ & 6165 & -782 & 6000 & 43 \\
\hline $\operatorname{Si}(10)$ & 8608 & 336 & 6127 & 35 \\
\hline $\operatorname{Si}(11)$ & 3112 & 537 & 5763 & 36 \\
\hline $\operatorname{Si}(12)$ & 5281 & 1756 & 5825 & 29 \\
\hline $\operatorname{Si}(13)$ & 5079 & 4227 & 3954 & 23 \\
\hline $\operatorname{Si}(14)$ & 4624 & 3250 & 3933 & 30 \\
\hline $\operatorname{Si}(15)$ & 6917 & 4480 & 4062 & 30 \\
\hline $\operatorname{Si}(16)$ & 3263 & 4977 & 4025 & 24 \\
\hline $\operatorname{Si}(17)$ & 1468 & 4936 & 3988 & 35 \\
\hline $\operatorname{Si}(18)$ & 4009 & 5882 & 4320 & 31 \\
\hline $\operatorname{Si}(19)$ & 6679 & 2550 & 986 & 31 \\
\hline $\operatorname{Si}(20)$ & 5992 & 1608 & 745 & 42 \\
\hline $\operatorname{Si}(21)$ & 8468 & 2617 & 933 & 40 \\
\hline $\operatorname{Si}(22)$ & 4791 & 3252 & 1067 & 34 \\
\hline $\operatorname{Si}(23)$ & 2975 & 2998 & 960 & 47 \\
\hline $\operatorname{Si}(24)$ & 5180 & 4238 & 1102 & 38 \\
\hline $\operatorname{Zr}(1)$ & 4276 & 2125 & 8475 & 28 \\
\hline $\operatorname{Zr}(2)$ & 5596 & 778 & 6580 & 26 \\
\hline $\operatorname{Zr}(3)$ & 4228 & 4669 & 3431 & 27 \\
\hline $\operatorname{Zr}(4)$ & 5846 & 2942 & 1600 & 29 \\
\hline
\end{tabular}


Table S13. Bond lengths $(\AA)$ and angles $\left({ }^{\circ}\right)$ for 3.

\begin{tabular}{|c|c|c|c|}
\hline $\mathrm{C}(1)-\mathrm{N}(1)$ & 1.4360 & $\mathrm{C}(43)-\mathrm{N}(8)$ & 1.4591 \\
\hline $\mathrm{C}(2)-\mathrm{N}(1)$ & 1.5059 & $\mathrm{C}(44)-\mathrm{N}(8)$ & 1.4556 \\
\hline $\mathrm{C}(3)-\mathrm{N}(2)$ & 1.4531 & $\mathrm{C}(45)-\mathrm{N}(9)$ & 1.4563 \\
\hline $\mathrm{C}(4)-\mathrm{N}(2)$ & 1.4616 & $\mathrm{C}(46)-\mathrm{N}(9)$ & 1.4414 \\
\hline $\mathrm{C}(5)-\mathrm{N}(3)$ & 1.4399 & $\mathrm{C}(47)-\operatorname{Si}(14)$ & 1.8975 \\
\hline $\mathrm{C}(6)-\mathrm{N}(3)$ & 1.4657 & $\mathrm{C}(48)-\mathrm{Si}(14)$ & 1.9130 \\
\hline $\mathrm{C}(7)-\mathrm{Si}(3)$ & 1.8980 & C(49)-Si(14) & 1.8927 \\
\hline $\mathrm{C}(8)-\operatorname{Si}(3)$ & 1.8981 & $\mathrm{C}(50)-\operatorname{Si}(15)$ & 1.9056 \\
\hline C(9)-Si(3) & 1.8995 & $\mathrm{C}(51)-\operatorname{Si}(15)$ & 1.9015 \\
\hline $\mathrm{C}(10)-\mathrm{Si}(4)$ & 1.9201 & $\mathrm{C}(52)-\operatorname{Si}(15)$ & 1.8752 \\
\hline $\mathrm{C}(11)-\mathrm{Si}(4)$ & 1.8940 & C(53)-Si(17) & 1.9105 \\
\hline C(12)-Si(4) & 1.8785 & $\mathrm{C}(54)-\mathrm{Si}(17)$ & 1.9224 \\
\hline$C(13)-C(14)$ & 1.5409 & $\mathrm{C}(55)-\mathrm{Si}(17)$ & 1.9052 \\
\hline C(13)-Si(1) & 1.9620 & C(56)-Si(18) & 1.8932 \\
\hline $\mathrm{C}(14)-\mathrm{Si}(2)$ & 1.9422 & $\mathrm{C}(57)-\mathrm{Si}(18)$ & 1.9227 \\
\hline$C(15)-\operatorname{Si}(5)$ & 1.9019 & $\mathrm{C}(58)-\operatorname{Si}(18)$ & 1.8932 \\
\hline$C(16)-\operatorname{Si}(5)$ & 1.9015 & $\mathrm{C}(59)-\mathrm{C}(60)$ & 1.5499 \\
\hline$C(17)-\operatorname{Si}(5)$ & 1.9355 & C(59)-Si(16) & 1.9285 \\
\hline $\mathrm{C}(18)-\mathrm{Si}(6)$ & 1.8918 & $\mathrm{C}(60)-\mathrm{Si}(13)$ & 1.9414 \\
\hline C(19)-Si(6) & 1.8907 & $\mathrm{C}(61)-\mathrm{N}(10)$ & 1.5824 \\
\hline $\mathrm{C}(20)-\mathrm{Si}(6)$ & 1.8980 & $\mathrm{C}(62)-\mathrm{N}(10)$ & 1.3169 \\
\hline $\mathrm{C}(21)-\mathrm{N}(4)$ & 1.4302 & $\mathrm{C}(63)-\mathrm{N}(11)$ & 1.4501 \\
\hline $\mathrm{C}(22)-\mathrm{N}(4)$ & 1.5037 & $\mathrm{C}(64)-\mathrm{N}(11)$ & 1.4380 \\
\hline $\mathrm{C}(23)-\mathrm{N}(5)$ & 1.4743 & $\mathrm{C}(65)-\mathrm{N}(12)$ & 1.5139 \\
\hline $\mathrm{C}(24)-\mathrm{N}(5)$ & 1.4747 & $\mathrm{C}(66)-\mathrm{N}(12)$ & 1.4472 \\
\hline $\mathrm{C}(25)-\mathrm{N}(6)$ & 1.4482 & $\mathrm{C}(67)-\operatorname{Si}(20)$ & 1.8915 \\
\hline $\mathrm{C}(26)-\mathrm{N}(6)$ & 1.4759 & $\mathrm{C}(68)-\mathrm{Si}(20)$ & 1.8909 \\
\hline $\mathrm{C}(27)-\mathrm{Si}(9)$ & 1.8784 & $C(69)-\operatorname{Si}(20)$ & 1.9098 \\
\hline $\mathrm{C}(28)-\mathrm{Si}(9)$ & 1.8963 & $\mathrm{C}(70)-\mathrm{Si}(21)$ & 1.9205 \\
\hline C(29)-Si(9) & 1.9413 & $\mathrm{C}(71)-\mathrm{Si}(21)$ & 1.8864 \\
\hline $\mathrm{C}(30)-\mathrm{Si}(10)$ & 1.8931 & $\mathrm{C}(72)-\mathrm{Si}(21)$ & 1.9307 \\
\hline $\mathrm{C}(31)-\mathrm{Si}(10)$ & 1.9408 & $\mathrm{C}(73)-\mathrm{C}(74)$ & 1.4922 \\
\hline$C(32)-\operatorname{Si}(10)$ & 1.9195 & $\mathrm{C}(73)-\mathrm{Si}(19)$ & 1.9630 \\
\hline$C(33)-C(34)$ & 1.5419 & $\mathrm{C}(74)-\mathrm{Si}(22)$ & 1.9189 \\
\hline C(33)-Si(7) & 1.9279 & $C(75)-\operatorname{Si}(23)$ & 1.8706 \\
\hline C(34)-Si(8) & 1.9413 & $C(76)-\operatorname{Si}(23)$ & 1.9201 \\
\hline $\mathrm{C}(35)-\mathrm{Si}(11)$ & 1.8866 & $\mathrm{C}(77)-\mathrm{Si}(23)$ & 1.8568 \\
\hline $\mathrm{C}(36)-\mathrm{Si}(11)$ & 1.9029 & $\mathrm{C}(78)-\mathrm{Si}(24)$ & 1.9071 \\
\hline $\mathrm{C}(37)-\operatorname{Si}(11)$ & 1.8891 & $\mathrm{C}(79)-\mathrm{Si}(24)$ & 1.8910 \\
\hline $\mathrm{C}(38)-\mathrm{Si}(12)$ & 1.9124 & $\mathrm{C}(80)-\mathrm{Si}(24)$ & 1.9244 \\
\hline $\mathrm{C}(39)-\mathrm{Si}(12)$ & 1.8952 & $\mathrm{C}(81)-\mathrm{O}(1)$ & 1.4126 \\
\hline $\mathrm{C}(40)-\mathrm{Si}(12)$ & 1.8859 & $\mathrm{C}(81)-\mathrm{C}(82)$ & 1.4956 \\
\hline $\mathrm{C}(41)-\mathrm{N}(7)$ & 1.4635 & $\mathrm{C}(82)-\mathrm{O}(2)$ & 1.4348 \\
\hline $\mathrm{C}(42)-\mathrm{N}(7)$ & 1.5080 & $\mathrm{C}(83)-\mathrm{O}(2)$ & 1.3908 \\
\hline
\end{tabular}




\begin{tabular}{|c|c|c|c|}
\hline $\mathrm{C}(83)-\mathrm{C}(84)$ & 1.4804 & $\mathrm{C}(144)-\mathrm{O}(22)$ & 1.4346 \\
\hline $\mathrm{C}(84)-\mathrm{O}(3)$ & 1.4728 & $C(144)-C(145)$ & 1.5069 \\
\hline $\mathrm{C}(85)-\mathrm{O}(3)$ & 1.4367 & $\mathrm{C}(145)-\mathrm{O}(23)$ & 1.4688 \\
\hline C(85)-C(86) & 1.4818 & $\mathrm{C}(146)-\mathrm{O}(23)$ & 1.4198 \\
\hline $\mathrm{C}(86)-\mathrm{O}(1) \# 1$ & 1.4123 & $C(146)-C(147)$ & 1.4862 \\
\hline C(87)-C(92) & 1.3729 & $\mathrm{C}(147)-\mathrm{O}(24)$ & 1.4423 \\
\hline $\mathrm{C}(87)-\mathrm{C}(88)$ & 1.3827 & $\mathrm{C}(148)-\mathrm{O}(24)$ & 1.4317 \\
\hline $\mathrm{C}(87)-\mathrm{C}(93)$ & 1.5601 & C(148)-C(149) & 1.5135 \\
\hline $\mathrm{C}(88)-\mathrm{C}(89)$ & 1.3505 & $\mathrm{C}(149)-\mathrm{O}(25)$ & 1.4252 \\
\hline $\mathrm{C}(89)-\mathrm{C}(90)$ & 1.4108 & $\mathrm{C}(150)-\mathrm{O}(25)$ & 1.4223 \\
\hline C(90)-C(91) & 1.4100 & $\mathrm{C}(151)-\mathrm{C}(152)$ & 1.2893 \\
\hline C(91)-C(92) & 1.3425 & $\mathrm{C}(151)-\mathrm{O}(26)$ & 1.4248 \\
\hline $\mathrm{C}(99)-\mathrm{O}(26)$ & 1.4024 & $\mathrm{C}(152)-\mathrm{O}(27)$ & 1.4952 \\
\hline C(99)-C(150) & 1.5632 & $\mathrm{C}(153)-\mathrm{O}(27)$ & 1.4415 \\
\hline $\mathrm{C}(118)-\mathrm{O}(16)$ & 1.4331 & $C(153)-C(154)$ & 1.5004 \\
\hline C(118)-C(119) & 1.4633 & $\mathrm{C}(154)-\mathrm{O}(22)$ & 1.4562 \\
\hline $\mathrm{C}(119)-\mathrm{O}(17)$ & 1.3765 & $\mathrm{C}(155)-\mathrm{O}(28)$ & 1.4580 \\
\hline $\mathrm{C}(120)-\mathrm{O}(17)$ & 1.4501 & $\mathrm{C}(155)-\mathrm{C}(156)$ & 1.5486 \\
\hline $\mathrm{C}(120)-\mathrm{C}(121)$ & 1.4616 & $\mathrm{C}(156)-\mathrm{O}(29)$ & 1.4490 \\
\hline $\mathrm{C}(121)-\mathrm{O}(18)$ & 1.3978 & $\mathrm{C}(157)-\mathrm{O}(29)$ & 1.3942 \\
\hline $\mathrm{C}(122)-\mathrm{O}(18)$ & 1.4389 & C(157)-C(158) & 1.5464 \\
\hline$C(122)-C(123)$ & 1.4415 & $\mathrm{C}(158)-\mathrm{O}(30)$ & 1.4532 \\
\hline $\mathrm{C}(123)-\mathrm{O}(19)$ & 1.4070 & $\mathrm{C}(159)-\mathrm{O}(30)$ & 1.4710 \\
\hline $\mathrm{C}(124)-\mathrm{O}(19)$ & 1.3955 & C(159)-C(160) & 1.5262 \\
\hline$C(124)-C(125)$ & 1.4367 & $\mathrm{C}(160)-\mathrm{O}(31)$ & 1.4363 \\
\hline $\mathrm{C}(125)-\mathrm{O}(20)$ & 1.4316 & $\mathrm{C}(161)-\mathrm{O}(31)$ & 1.3884 \\
\hline $\mathrm{C}(126)-\mathrm{O}(20)$ & 1.4376 & $\mathrm{C}(161)-\mathrm{C}(162)$ & 1.5226 \\
\hline$C(126)-C(127)$ & 1.4562 & $\mathrm{C}(162)-\mathrm{O}(32)$ & 1.4234 \\
\hline $\mathrm{C}(127)-\mathrm{O}(21)$ & 1.4278 & $\mathrm{C}(163)-\mathrm{O}(32)$ & 1.4006 \\
\hline $\mathrm{C}(128)-\mathrm{O}(21)$ & 1.4400 & $\mathrm{C}(163)-\mathrm{C}(164)$ & 1.5634 \\
\hline $\mathrm{C}(128)-\mathrm{C}(129)$ & 1.4581 & $\mathrm{C}(164)-\mathrm{O}(33)$ & 1.4235 \\
\hline $\mathrm{C}(129)-\mathrm{O}(16)$ & 1.3977 & $\mathrm{C}(165)-\mathrm{O}(33)$ & 1.4447 \\
\hline$C(130)-C(131)$ & 1.3597 & $C(165)-C(166)$ & 1.5136 \\
\hline$C(130)-C(135)$ & 1.4005 & $\mathrm{C}(166)-\mathrm{O}(28)$ & 1.4036 \\
\hline$C(130)-C(136)$ & 1.5466 & $\mathrm{C}(167)-\mathrm{O}(36) \# 2$ & 1.4337 \\
\hline $\mathrm{C}(131)-\mathrm{C}(132)$ & 1.3622 & C(167)-C(168) & 1.4550 \\
\hline $\mathrm{C}(132)-\mathrm{C}(133)$ & 1.3846 & $\mathrm{C}(168)-\mathrm{O}(34)$ & 1.4437 \\
\hline$C(133)-C(134)$ & 1.3677 & $\mathrm{C}(169)-\mathrm{O}(34)$ & 1.4544 \\
\hline $\mathrm{C}(134)-\mathrm{C}(135)$ & 1.4346 & C(169)-C(170) & 1.4685 \\
\hline$C(137)-C(142)$ & 1.3799 & $\mathrm{C}(170)-\mathrm{O}(35)$ & 1.4620 \\
\hline $\mathrm{C}(137)-\mathrm{C}(138)$ & 1.4025 & $\mathrm{C}(171)-\mathrm{O}(35)$ & 1.4158 \\
\hline C(137)-C(143) & 1.5256 & $\mathrm{C}(171)-\mathrm{C}(172)$ & 1.4620 \\
\hline C(138)-C(139) & 1.4133 & $\mathrm{C}(172)-\mathrm{O}(36)$ & 1.4580 \\
\hline$C(139)-C(140)$ & 1.3877 & $\mathrm{C}(173)-\mathrm{C}(174)$ & 1.3858 \\
\hline$C(140)-C(141)$ & 1.3855 & $\mathrm{C}(173)-\mathrm{C}(178)$ & 1.3922 \\
\hline$C(141)-C(142)$ & 1.4275 & $\mathrm{C}(173)-\mathrm{C}(179)$ & 1.5349 \\
\hline
\end{tabular}




\begin{tabular}{|c|c|c|c|}
\hline$C(174)-C(175)$ & 1.3541 & $\mathrm{~K}(1)-\mathrm{O}(1)$ & 2.8051 \\
\hline $\mathrm{C}(175)-\mathrm{C}(176)$ & 1.3988 & $\mathrm{~K}(1)-\mathrm{C}(90) \# 1$ & 3.4787 \\
\hline $\mathrm{C}(176)-\mathrm{C}(177)$ & 1.4245 & $\mathrm{~K}(2)-\mathrm{O}(28)$ & 2.7402 \\
\hline $\mathrm{C}(177)-\mathrm{C}(178)$ & 1.3757 & $\mathrm{~K}(2)-\mathrm{O}(26)$ & 2.8364 \\
\hline $\mathrm{C}(228)-\mathrm{O}(101)$ & 1.3694 & $\mathrm{~K}(2)-\mathrm{O}(23)$ & 2.8491 \\
\hline $\mathrm{C}(228)-\mathrm{C}(229)$ & 1.4251 & $\mathrm{~K}(2)-\mathrm{O}(27)$ & 2.8553 \\
\hline $\mathrm{C}(229)-\mathrm{O}(102)$ & 1.4406 & $\mathrm{~K}(2)-\mathrm{O}(22)$ & 2.9153 \\
\hline $\mathrm{C}(230)-\mathrm{C}(231)$ & 1.3587 & $\mathrm{~K}(2)-\mathrm{O}(25)$ & 2.9204 \\
\hline $\mathrm{C}(230)-\mathrm{O}(102)$ & 1.4311 & $\mathrm{~K}(2)-\mathrm{O}(24)$ & 2.9462 \\
\hline $\mathrm{C}(231)-\mathrm{O}(103)$ & 1.5741 & $\mathrm{~K}(2)-\mathrm{O}(33)$ & 2.9917 \\
\hline $\mathrm{C}(232)-\mathrm{C}(233)$ & 1.2802 & $\mathrm{~K}(3)-\mathrm{O}(21)$ & 2.7568 \\
\hline $\mathrm{C}(232)-\mathrm{O}(103)$ & 1.3452 & $\mathrm{~K}(3)-\mathrm{O}(16)$ & 2.7704 \\
\hline $\mathrm{C}(233)-\mathrm{O}(104)$ & 1.5855 & $\mathrm{~K}(3)-\mathrm{O}(19)$ & 2.8097 \\
\hline $\mathrm{C}(234)-\mathrm{O}(104)$ & 1.4363 & $\mathrm{~K}(3)-\mathrm{O}(18)$ & 2.8102 \\
\hline $\mathrm{C}(234)-\mathrm{C}(235)$ & 1.4453 & $\mathrm{~K}(3)-\mathrm{O}(20)$ & 2.8155 \\
\hline $\mathrm{C}(235)-\mathrm{O}(105)$ & 1.3568 & $\mathrm{~K}(3)-\mathrm{O}(17)$ & 2.8266 \\
\hline $\mathrm{C}(236)-\mathrm{C}(237)$ & 1.3330 & $\mathrm{~K}(4)-\mathrm{O}(108)$ & 2.8005 \\
\hline $\mathrm{C}(236)-\mathrm{O}(105)$ & 1.4252 & $\mathrm{~K}(4)-\mathrm{O}(102)$ & 2.8137 \\
\hline $\mathrm{C}(237)-\mathrm{O}(106)$ & 1.5249 & $\mathrm{~K}(4)-\mathrm{O}(105)$ & 2.8335 \\
\hline $\mathrm{C}(238)-\mathrm{O}(106)$ & 1.3575 & $\mathrm{~K}(4)-\mathrm{O}(101)$ & 2.8341 \\
\hline $\mathrm{C}(238)-\mathrm{C}(239)$ & 1.4153 & $\mathrm{~K}(4)-\mathrm{O}(106)$ & 2.8790 \\
\hline $\mathrm{C}(239)-\mathrm{O}(101)$ & 1.4470 & $\mathrm{~K}(4)-\mathrm{O}(103)$ & 2.9065 \\
\hline $\mathrm{C}(240)-\mathrm{O}(108)$ & 1.4100 & $\mathrm{~K}(4)-\mathrm{O}(104)$ & 2.9170 \\
\hline$C(240)-C(241)$ & 1.5016 & $\mathrm{~K}(4)-\mathrm{O}(107)$ & 3.0801 \\
\hline $\mathrm{C}(241)-\mathrm{O}(107)$ & 1.4344 & $\mathrm{~K}(5)-\mathrm{O}(34) \# 2$ & 2.7549 \\
\hline $\mathrm{C}(241)-\mathrm{K}(4)$ & 3.5231 & $\mathrm{~K}(5)-\mathrm{O}(34)$ & 2.7549 \\
\hline $\mathrm{C}(247)-\mathrm{O}(108)$ & 1.4496 & $\mathrm{~K}(5)-\mathrm{O}(36) \# 2$ & 2.7756 \\
\hline $\mathrm{C}(247)-\mathrm{C}(248)$ & 1.5003 & $\mathrm{~K}(5)-\mathrm{O}(36)$ & 2.7756 \\
\hline $\mathrm{C}(248)-\mathrm{O}(112)$ & 1.4252 & $\mathrm{~K}(5)-\mathrm{O}(35) \# 2$ & 2.8209 \\
\hline $\mathrm{C}(249)-\mathrm{O}(107)$ & 1.4205 & $\mathrm{~K}(5)-\mathrm{O}(35)$ & 2.8209 \\
\hline $\mathrm{C}(249)-\mathrm{C}(250)$ & 1.5207 & $\mathrm{~K}(5)-\mathrm{C}(176) \# 2$ & 3.3826 \\
\hline $\mathrm{C}(250)-\mathrm{O}(113)$ & 1.3662 & $\mathrm{~K}(5)-\mathrm{C}(177) \# 2$ & 3.5119 \\
\hline $\mathrm{C}(251)-\mathrm{O}(112)$ & 1.4708 & $\mathrm{~N}(1)-\operatorname{Zr}(1)$ & 2.0663 \\
\hline $\mathrm{C}(251)-\mathrm{C}(252)$ & 1.5130 & $\mathrm{~N}(2)-\operatorname{Zr}(1)$ & 2.0544 \\
\hline $\mathrm{C}(252)-\mathrm{O}(117)$ & 1.3660 & $\mathrm{~N}(3)-\operatorname{Zr}(1)$ & 2.0729 \\
\hline$C(253)-C(254)$ & 1.4223 & $\mathrm{~N}(4)-\operatorname{Zr}(2)$ & 2.1055 \\
\hline $\mathrm{C}(253)-\mathrm{O}(113)$ & 1.4435 & $N(5)-Z r(2)$ & 2.0845 \\
\hline $\mathrm{C}(254)-\mathrm{O}(114)$ & 1.4612 & $\mathrm{~N}(6)-\operatorname{Zr}(2)$ & 2.0602 \\
\hline $\mathrm{C}(255)-\mathrm{O}(114)$ & 1.3119 & $N(7)-\operatorname{Zr}(3)$ & 2.0869 \\
\hline $\mathrm{C}(255)-\mathrm{C}(259)$ & 1.4405 & $\mathrm{~N}(8)-\operatorname{Zr}(3)$ & 2.0492 \\
\hline $\mathrm{C}(259)-\mathrm{O}(117)$ & 1.4659 & $\mathrm{~N}(9)-\operatorname{Zr}(3)$ & 2.0955 \\
\hline $\mathrm{K}(1)-\mathrm{O}(3)$ & 2.7569 & $\mathrm{~N}(10)-\operatorname{Zr}(4)$ & 2.0677 \\
\hline $\mathrm{K}(1)-\mathrm{O}(3) \# 1$ & 2.7570 & $\mathrm{~N}(11)-\operatorname{Zr}(4)$ & 2.0963 \\
\hline $\mathrm{K}(1)-\mathrm{O}(2)$ & 2.7966 & $\mathrm{~N}(12)-\mathrm{Zr}(4)$ & 2.0320 \\
\hline $\mathrm{K}(1)-\mathrm{O}(2) \# 1$ & 2.7966 & $\mathrm{O}(1)-\mathrm{C}(86) \# 1$ & 1.4123 \\
\hline $\mathrm{K}(1)-\mathrm{O}(1) \# 1$ & 2.8051 & $\mathrm{O}(36)-\mathrm{C}(167) \# 2$ & 1.4337 \\
\hline
\end{tabular}




\begin{tabular}{|c|c|c|c|}
\hline $\operatorname{Si}(1)-\operatorname{Si}(4)$ & 2.3522 & $\operatorname{Si}(13)-\operatorname{Si}(14)$ & 2.366 \\
\hline $\mathrm{Si}(1)-\mathrm{Si}(3)$ & 2.3694 & $\operatorname{Si}(13)-\operatorname{Si}(15)$ & 2.368 \\
\hline $\mathrm{Si}(1)-\operatorname{Zr}(1)$ & 2.8862 & $\operatorname{Si}(13)-\operatorname{Zr}(3)$ & 2.906 \\
\hline $\operatorname{Si}(2)-\operatorname{Si}(5)$ & 2.3339 & $\operatorname{Si}(16)-\operatorname{Si}(17)$ & 2.328 \\
\hline $\operatorname{Si}(2)-\operatorname{Si}(6)$ & 2.3540 & $\operatorname{Si}(16)-\operatorname{Si}(18)$ & 2.351 \\
\hline $\mathrm{Si}(2)-\mathrm{Zr}(1)$ & 2.8655 & $\operatorname{Si}(16)-\operatorname{Zr}(3)$ & \\
\hline $\operatorname{Si}(7)-\operatorname{Si}(10)$ & 2.3515 & $\operatorname{Si}(19)-\operatorname{Si}(21)$ & \\
\hline $\operatorname{Si}(7)-\operatorname{Si}(9)$ & 2.3665 & $\operatorname{Si}(19)-\operatorname{Si}(20)$ & 2.346 \\
\hline $\operatorname{Si}(7)-\operatorname{Zr}(2)$ & 2.9079 & $\operatorname{Si}(19)-\operatorname{Zr}(4)$ & \\
\hline $\operatorname{Si}(8)-\operatorname{Si}(11)$ & 2.3342 & $\operatorname{Si}(22)-\operatorname{Si}(23)$ & 2.341 \\
\hline $\operatorname{Si}(8)-\operatorname{Si}(12)$ & 2.3530 & $\operatorname{Si}(22)-\operatorname{Si}(24)$ & \\
\hline $\operatorname{Si}(8)-\operatorname{Zr}(2)$ & 2.8917 & $\operatorname{Si}(22)-\operatorname{Zr}(4)$ & 2.8 \\
\hline$C(14)-C(13)-\operatorname{Si}(1)$ & 112.0 & $\mathrm{O}(21)-\mathrm{C}(128)-\mathrm{C}(129)$ & 109.3 \\
\hline$C(13)-C(14)-\operatorname{Si}(2)$ & 106.8 & $\mathrm{O}(16)-\mathrm{C}(129)-\mathrm{C}(128)$ & 113.0 \\
\hline $\mathrm{C}(34)-\mathrm{C}(33)-\mathrm{Si}(7)$ & 111.2 & $\mathrm{C}(131)-\mathrm{C}(130)-\mathrm{C}(135)$ & 120.3 \\
\hline C(33)-C(34)-Si(8) & 111.1 & $C(131)-C(130)-C(136)$ & 122.2 \\
\hline $\mathrm{C}(60)-\mathrm{C}(59)-\mathrm{Si}(16)$ & 109.1 & $C(135)-C(130)-C(136)$ & 117.5 \\
\hline $\mathrm{C}(59)-\mathrm{C}(60)-\mathrm{Si}(13)$ & 110.1 & $C(130)-C(131)-C(132)$ & 121.3 \\
\hline$C(74)-C(73)-S i(19)$ & 108.3 & $C(131)-C(132)-C(133)$ & 119.9 \\
\hline $\mathrm{C}(73)-\mathrm{C}(74)-\mathrm{Si}(22)$ & 113.2 & $C(134)-C(133)-C(132)$ & 121.2 \\
\hline $\mathrm{O}(1)-\mathrm{C}(81)-\mathrm{C}(82)$ & 109.6 & $C(133)-C(134)-C(135)$ & 118.6 \\
\hline $\mathrm{O}(2)-\mathrm{C}(82)-\mathrm{C}(81)$ & 109.4 & $C(130)-C(135)-C(134)$ & 118.5 \\
\hline $\mathrm{O}(2)-\mathrm{C}(83)-\mathrm{C}(84)$ & 108.8 & $\mathrm{C}(142)-\mathrm{C}(137)-\mathrm{C}(138)$ & 120.9 \\
\hline $\mathrm{O}(3)-\mathrm{C}(84)-\mathrm{C}(83)$ & 109.4 & $\mathrm{C}(142)-\mathrm{C}(137)-\mathrm{C}(143)$ & 119.3 \\
\hline $\mathrm{O}(3)-\mathrm{C}(85)-\mathrm{C}(86)$ & 107.6 & $C(138)-C(137)-C(143)$ & 119.7 \\
\hline $\mathrm{O}(1) \# 1-\mathrm{C}(86)-\mathrm{C}(85)$ & 109.0 & $\mathrm{C}(137)-\mathrm{C}(138)-\mathrm{C}(139)$ & 118.1 \\
\hline $\mathrm{C}(92)-\mathrm{C}(87)-\mathrm{C}(88)$ & 120.6 & $C(140)-C(139)-C(138)$ & 121.6 \\
\hline $\mathrm{C}(92)-\mathrm{C}(87)-\mathrm{C}(93)$ & 119.1 & $C(141)-C(140)-C(139)$ & 119.8 \\
\hline $\mathrm{C}(88)-\mathrm{C}(87)-\mathrm{C}(93)$ & 120.4 & $\mathrm{C}(140)-\mathrm{C}(141)-\mathrm{C}(142)$ & 119.4 \\
\hline $\mathrm{C}(89)-\mathrm{C}(88)-\mathrm{C}(87)$ & 122.7 & $\mathrm{C}(137)-\mathrm{C}(142)-\mathrm{C}(141)$ & 120.1 \\
\hline $\mathrm{C}(88)-\mathrm{C}(89)-\mathrm{C}(90)$ & 117.5 & $\mathrm{O}(22)-\mathrm{C}(144)-\mathrm{C}(145)$ & 106.8 \\
\hline $\mathrm{C}(91)-\mathrm{C}(90)-\mathrm{C}(89)$ & 118.4 & $\mathrm{O}(23)-\mathrm{C}(145)-\mathrm{C}(144)$ & 108.9 \\
\hline $\mathrm{C}(92)-\mathrm{C}(91)-\mathrm{C}(90)$ & 122.9 & $\mathrm{O}(23)-\mathrm{C}(146)-\mathrm{C}(147)$ & 111.7 \\
\hline C(91)-C(92)-C(87) & 117.9 & $\mathrm{O}(24)-\mathrm{C}(147)-\mathrm{C}(146)$ & 110.3 \\
\hline $\mathrm{O}(26)-\mathrm{C}(99)-\mathrm{C}(150)$ & 106.8 & $\mathrm{O}(24)-\mathrm{C}(148)-\mathrm{C}(149)$ & 107.2 \\
\hline $\mathrm{O}(16)-\mathrm{C}(118)-\mathrm{C}(119)$ & 111.3 & $\mathrm{O}(25)-\mathrm{C}(149)-\mathrm{C}(148)$ & 109.0 \\
\hline $\mathrm{O}(17)-\mathrm{C}(119)-\mathrm{C}(118)$ & 110.2 & $\mathrm{O}(25)-\mathrm{C}(150)-\mathrm{C}(99)$ & 105.8 \\
\hline $\mathrm{O}(17)-\mathrm{C}(120)-\mathrm{C}(121)$ & 111.0 & $\mathrm{C}(152)-\mathrm{C}(151)-\mathrm{O}(26)$ & 121.3 \\
\hline $\mathrm{O}(18)-\mathrm{C}(121)-\mathrm{C}(120)$ & 109.9 & $\mathrm{C}(151)-\mathrm{C}(152)-\mathrm{O}(27)$ & 118.4 \\
\hline $\mathrm{O}(18)-\mathrm{C}(122)-\mathrm{C}(123)$ & 110.1 & $\mathrm{O}(27)-\mathrm{C}(153)-\mathrm{C}(154)$ & 108.4 \\
\hline $\mathrm{O}(19)-\mathrm{C}(123)-\mathrm{C}(122)$ & 112.5 & $\mathrm{O}(22)-\mathrm{C}(154)-\mathrm{C}(153)$ & 106.9 \\
\hline $\mathrm{O}(19)-\mathrm{C}(124)-\mathrm{C}(125)$ & 111.3 & $\mathrm{O}(28)-\mathrm{C}(155)-\mathrm{C}(156)$ & 113.0 \\
\hline $\mathrm{O}(20)-\mathrm{C}(125)-\mathrm{C}(124)$ & 108.2 & $\mathrm{O}(29)-\mathrm{C}(156)-\mathrm{C}(155)$ & 107.2 \\
\hline $\mathrm{O}(20)-\mathrm{C}(126)-\mathrm{C}(127)$ & 110.6 & $\mathrm{O}(29)-\mathrm{C}(157)-\mathrm{C}(158)$ & 106.6 \\
\hline $\mathrm{O}(21)-\mathrm{C}(127)-\mathrm{C}(126)$ & 110.6 & $\mathrm{O}(30)-\mathrm{C}(158)-\mathrm{C}(157)$ & 103.6 \\
\hline
\end{tabular}




\begin{tabular}{|c|c|c|c|}
\hline $\mathrm{O}(30)-\mathrm{C}(159)-\mathrm{C}(160)$ & 110.2 & $\mathrm{O}(114)-\mathrm{C}(255)-\mathrm{C}(259)$ & 111.0 \\
\hline $\mathrm{O}(31)-\mathrm{C}(160)-\mathrm{C}(159)$ & 108.4 & $\mathrm{C}(255)-\mathrm{C}(259)-\mathrm{O}(117)$ & 113.7 \\
\hline $\mathrm{O}(31)-\mathrm{C}(161)-\mathrm{C}(162)$ & 107.2 & $\mathrm{O}(3)-\mathrm{K}(1)-\mathrm{O}(3) \# 1$ & 180.0 \\
\hline $\mathrm{O}(32)-\mathrm{C}(162)-\mathrm{C}(161)$ & 107.2 & $\mathrm{O}(3)-\mathrm{K}(1)-\mathrm{O}(2)$ & 60.8 \\
\hline $\mathrm{O}(32)-\mathrm{C}(163)-\mathrm{C}(164)$ & 105.3 & $\mathrm{O}(3) \# 1-\mathrm{K}(1)-\mathrm{O}(2)$ & 119.2 \\
\hline $\mathrm{O}(33)-\mathrm{C}(164)-\mathrm{C}(163)$ & 105.6 & $\mathrm{O}(3)-\mathrm{K}(1)-\mathrm{O}(2) \# 1$ & 119.2 \\
\hline $\mathrm{O}(33)-\mathrm{C}(165)-\mathrm{C}(166)$ & 112.0 & $\mathrm{O}(3) \# 1-\mathrm{K}(1)-\mathrm{O}(2) \# 1$ & 60.8 \\
\hline $\mathrm{O}(28)-\mathrm{C}(166)-\mathrm{C}(165)$ & 107.8 & $\mathrm{O}(2)-\mathrm{K}(1)-\mathrm{O}(2) \# 1$ & 180.0 \\
\hline $\mathrm{O}(36) \# 2-\mathrm{C}(167)-\mathrm{C}(168)$ & 108.7 & $\mathrm{O}(3)-\mathrm{K}(1)-\mathrm{O}(1) \# 1$ & 60.5 \\
\hline $\mathrm{O}(34)-\mathrm{C}(168)-\mathrm{C}(167)$ & 108.4 & $\mathrm{O}(3) \# 1-\mathrm{K}(1)-\mathrm{O}(1) \# 1$ & 119.5 \\
\hline $\mathrm{O}(34)-\mathrm{C}(169)-\mathrm{C}(170)$ & 107.7 & $\mathrm{O}(2)-\mathrm{K}(1)-\mathrm{O}(1) \# 1$ & 119.8 \\
\hline $\mathrm{O}(35)-\mathrm{C}(170)-\mathrm{C}(169)$ & 109.9 & $\mathrm{O}(2) \# 1-\mathrm{K}(1)-\mathrm{O}(1) \# 1$ & 60.2 \\
\hline $\mathrm{O}(35)-\mathrm{C}(171)-\mathrm{C}(172)$ & 107.6 & $\mathrm{O}(3)-\mathrm{K}(1)-\mathrm{O}(1)$ & 119.5 \\
\hline $\mathrm{O}(36)-\mathrm{C}(172)-\mathrm{C}(171)$ & 108.4 & $\mathrm{O}(3) \# 1-\mathrm{K}(1)-\mathrm{O}(1)$ & 60.5 \\
\hline $\mathrm{C}(174)-\mathrm{C}(173)-\mathrm{C}(178)$ & 119.3 & $\mathrm{O}(2)-\mathrm{K}(1)-\mathrm{O}(1)$ & 60.2 \\
\hline$C(174)-C(173)-C(179)$ & 122.5 & $\mathrm{O}(2) \# 1-\mathrm{K}(1)-\mathrm{O}(1)$ & 119.8 \\
\hline $\mathrm{C}(178)-\mathrm{C}(173)-\mathrm{C}(179)$ & 118.2 & $\mathrm{O}(1) \# 1-\mathrm{K}(1)-\mathrm{O}(1)$ & 180.0 \\
\hline$C(175)-C(174)-C(173)$ & 121.7 & $\mathrm{O}(3)-\mathrm{K}(1)-\mathrm{C}(90) \# 1$ & 102.0 \\
\hline $\mathrm{C}(174)-\mathrm{C}(175)-\mathrm{C}(176)$ & 120.0 & $\mathrm{O}(3) \# 1-\mathrm{K}(1)-\mathrm{C}(90) \# 1$ & 78.0 \\
\hline $\mathrm{C}(175)-\mathrm{C}(176)-\mathrm{C}(177)$ & 118.8 & $\mathrm{O}(2)-\mathrm{K}(1)-\mathrm{C}(90) \# 1$ & 81.3 \\
\hline $\mathrm{C}(178)-\mathrm{C}(177)-\mathrm{C}(176)$ & 119.7 & $\mathrm{O}(2) \# 1-\mathrm{K}(1)-\mathrm{C}(90) \# 1$ & 98.7 \\
\hline $\mathrm{C}(177)-\mathrm{C}(178)-\mathrm{C}(173)$ & 120.3 & $\mathrm{O}(1) \# 1-\mathrm{K}(1)-\mathrm{C}(90) \# 1$ & 98.8 \\
\hline $\mathrm{O}(101)-\mathrm{C}(228)-\mathrm{C}(229)$ & 110.3 & $\mathrm{O}(1)-\mathrm{K}(1)-\mathrm{C}(90) \# 1$ & 81.2 \\
\hline $\mathrm{C}(228)-\mathrm{C}(229)-\mathrm{O}(102)$ & 110.7 & $\mathrm{O}(28)-\mathrm{K}(2)-\mathrm{O}(26)$ & 120.4 \\
\hline $\mathrm{C}(231)-\mathrm{C}(230)-\mathrm{O}(102)$ & 102.8 & $\mathrm{O}(28)-\mathrm{K}(2)-\mathrm{O}(23)$ & 77.2 \\
\hline $\mathrm{C}(230)-\mathrm{C}(231)-\mathrm{O}(103)$ & 114.3 & $\mathrm{O}(26)-\mathrm{K}(2)-\mathrm{O}(23)$ & 160.1 \\
\hline $\mathrm{C}(233)-\mathrm{C}(232)-\mathrm{O}(103)$ & 108.1 & $\mathrm{O}(28)-\mathrm{K}(2)-\mathrm{O}(27)$ & 138.6 \\
\hline $\mathrm{C}(232)-\mathrm{C}(233)-\mathrm{O}(104)$ & 123.1 & $\mathrm{O}(26)-\mathrm{K}(2)-\mathrm{O}(27)$ & 58.7 \\
\hline $\mathrm{O}(104)-\mathrm{C}(234)-\mathrm{C}(235)$ & 105.4 & $\mathrm{O}(23)-\mathrm{K}(2)-\mathrm{O}(27)$ & 115.6 \\
\hline $\mathrm{O}(105)-\mathrm{C}(235)-\mathrm{C}(234)$ & 110.8 & $\mathrm{O}(28)-\mathrm{K}(2)-\mathrm{O}(22)$ & 128.2 \\
\hline $\mathrm{C}(237)-\mathrm{C}(236)-\mathrm{O}(105)$ & 111.0 & $\mathrm{O}(26)-\mathrm{K}(2)-\mathrm{O}(22)$ & 108.8 \\
\hline $\mathrm{C}(236)-\mathrm{C}(237)-\mathrm{O}(106)$ & 110.8 & $\mathrm{O}(23)-\mathrm{K}(2)-\mathrm{O}(22)$ & 58.4 \\
\hline $\mathrm{O}(106)-\mathrm{C}(238)-\mathrm{C}(239)$ & 110.9 & $\mathrm{O}(27)-\mathrm{K}(2)-\mathrm{O}(22)$ & 58.8 \\
\hline $\mathrm{C}(238)-\mathrm{C}(239)-\mathrm{O}(101)$ & 112.3 & $\mathrm{O}(28)-\mathrm{K}(2)-\mathrm{O}(25)$ & 102.5 \\
\hline $\mathrm{O}(108)-\mathrm{C}(240)-\mathrm{C}(241)$ & 106.9 & $\mathrm{O}(26)-\mathrm{K}(2)-\mathrm{O}(25)$ & 58.5 \\
\hline $\mathrm{O}(107)-\mathrm{C}(241)-\mathrm{C}(240)$ & 115.3 & $\mathrm{O}(23)-\mathrm{K}(2)-\mathrm{O}(25)$ & 111.3 \\
\hline $\mathrm{O}(107)-\mathrm{C}(241)-\mathrm{K}(4)$ & 60.5 & $\mathrm{O}(27)-\mathrm{K}(2)-\mathrm{O}(25)$ & 107.8 \\
\hline $\mathrm{C}(240)-\mathrm{C}(241)-\mathrm{K}(4)$ & 87.6 & $\mathrm{O}(22)-\mathrm{K}(2)-\mathrm{O}(25)$ & 116.8 \\
\hline $\mathrm{O}(108)-\mathrm{C}(247)-\mathrm{C}(248)$ & 114.2 & $\mathrm{O}(28)-\mathrm{K}(2)-\mathrm{O}(24)$ & 72.1 \\
\hline $\mathrm{O}(112)-\mathrm{C}(248)-\mathrm{C}(247)$ & 105.6 & $\mathrm{O}(26)-\mathrm{K}(2)-\mathrm{O}(24)$ & 115.2 \\
\hline $\mathrm{O}(107)-\mathrm{C}(249)-\mathrm{C}(250)$ & 107.9 & $\mathrm{O}(23)-\mathrm{K}(2)-\mathrm{O}(24)$ & 58.6 \\
\hline $\mathrm{O}(113)-\mathrm{C}(250)-\mathrm{C}(249)$ & 107.2 & $\mathrm{O}(27)-\mathrm{K}(2)-\mathrm{O}(24)$ & 149.1 \\
\hline $\mathrm{O}(112)-\mathrm{C}(251)-\mathrm{C}(252)$ & 104.2 & $\mathrm{O}(22)-\mathrm{K}(2)-\mathrm{O}(24)$ & 101.8 \\
\hline $\mathrm{O}(117)-\mathrm{C}(252)-\mathrm{C}(251)$ & 106.5 & $\mathrm{O}(25)-\mathrm{K}(2)-\mathrm{O}(24)$ & 56.7 \\
\hline $\mathrm{C}(254)-\mathrm{C}(253)-\mathrm{O}(113)$ & 107.3 & $\mathrm{O}(28)-\mathrm{K}(2)-\mathrm{O}(33)$ & 58.3 \\
\hline $\mathrm{C}(253)-\mathrm{C}(254)-\mathrm{O}(114)$ & 112.9 & $\mathrm{O}(26)-\mathrm{K}(2)-\mathrm{O}(33)$ & 116.2 \\
\hline
\end{tabular}




\begin{tabular}{|c|c|c|c|}
\hline $\mathrm{O}(23)-\mathrm{K}(2)-\mathrm{O}(33)$ & 80.1 & $\mathrm{O}(103)-\mathrm{K}(4)-\mathrm{O}(107)$ & 132.8 \\
\hline $\mathrm{O}(27)-\mathrm{K}(2)-\mathrm{O}(33)$ & 84.1 & $\mathrm{O}(104)-\mathrm{K}(4)-\mathrm{O}(107)$ & 127.8 \\
\hline $\mathrm{O}(22)-\mathrm{K}(2)-\mathrm{O}(33)$ & 87.1 & $\mathrm{O}(108)-\mathrm{K}(4)-\mathrm{C}(241)$ & 41.5 \\
\hline $\mathrm{O}(25)-\mathrm{K}(2)-\mathrm{O}(33)$ & 156.1 & $\mathrm{O}(102)-\mathrm{K}(4)-\mathrm{C}(241)$ & 127.7 \\
\hline $\mathrm{O}(24)-\mathrm{K}(2)-\mathrm{O}(33)$ & 121.1 & $\mathrm{O}(105)-\mathrm{K}(4)-\mathrm{C}(241)$ & 74.3 \\
\hline $\mathrm{O}(21)-\mathrm{K}(3)-\mathrm{O}(16)$ & 61.5 & $\mathrm{O}(101)-\mathrm{K}(4)-\mathrm{C}(241)$ & 100.5 \\
\hline $\mathrm{O}(21)-\mathrm{K}(3)-\mathrm{O}(19)$ & 118.4 & $\mathrm{O}(106)-\mathrm{K}(4)-\mathrm{C}(241)$ & 84.1 \\
\hline $\mathrm{O}(16)-\mathrm{K}(3)-\mathrm{O}(19)$ & 179.7 & $\mathrm{O}(103)-\mathrm{K}(4)-\mathrm{C}(241)$ & 121.9 \\
\hline $\mathrm{O}(21)-\mathrm{K}(3)-\mathrm{O}(18)$ & 174.9 & $\mathrm{O}(104)-\mathrm{K}(4)-\mathrm{C}(241)$ & 104.1 \\
\hline $\mathrm{O}(16)-\mathrm{K}(3)-\mathrm{O}(18)$ & 119.1 & $\mathrm{O}(107)-\mathrm{K}(4)-\mathrm{C}(241)$ & 23.9 \\
\hline $\mathrm{O}(19)-\mathrm{K}(3)-\mathrm{O}(18)$ & 61.0 & $\mathrm{O}(34) \# 2-\mathrm{K}(5)-\mathrm{O}(34)$ & 180.0 \\
\hline $\mathrm{O}(21)-\mathrm{K}(3)-\mathrm{O}(20)$ & 61.1 & $\mathrm{O}(34) \# 2-\mathrm{K}(5)-\mathrm{O}(36) \# 2$ & 118.6 \\
\hline $\mathrm{O}(16)-\mathrm{K}(3)-\mathrm{O}(20)$ & 120.7 & $\mathrm{O}(34)-\mathrm{K}(5)-\mathrm{O}(36) \# 2$ & 61.4 \\
\hline $\mathrm{O}(19)-\mathrm{K}(3)-\mathrm{O}(20)$ & 59.2 & $\mathrm{O}(34) \# 2-\mathrm{K}(5)-\mathrm{O}(36)$ & 61.4 \\
\hline $\mathrm{O}(18)-\mathrm{K}(3)-\mathrm{O}(20)$ & 119.4 & $\mathrm{O}(34)-\mathrm{K}(5)-\mathrm{O}(36)$ & 118.6 \\
\hline $\mathrm{O}(21)-\mathrm{K}(3)-\mathrm{O}(17)$ & 119.4 & $\mathrm{O}(36) \# 2-\mathrm{K}(5)-\mathrm{O}(36)$ & 180.0 \\
\hline $\mathrm{O}(16)-\mathrm{K}(3)-\mathrm{O}(17)$ & 60.1 & $\mathrm{O}(34) \# 2-\mathrm{K}(5)-\mathrm{O}(35) \# 2$ & 60.8 \\
\hline $\mathrm{O}(19)-\mathrm{K}(3)-\mathrm{O}(17)$ & 120.1 & $\mathrm{O}(34)-\mathrm{K}(5)-\mathrm{O}(35) \# 2$ & 119.2 \\
\hline $\mathrm{O}(18)-\mathrm{K}(3)-\mathrm{O}(17)$ & 60.0 & $\mathrm{O}(36) \# 2-\mathrm{K}(5)-\mathrm{O}(35) \# 2$ & 59.2 \\
\hline $\mathrm{O}(20)-\mathrm{K}(3)-\mathrm{O}(17)$ & 178.9 & $\mathrm{O}(36)-\mathrm{K}(5)-\mathrm{O}(35) \# 2$ & 120.8 \\
\hline $\mathrm{O}(108)-\mathrm{K}(4)-\mathrm{O}(102)$ & 107.2 & $\mathrm{O}(34) \# 2-\mathrm{K}(5)-\mathrm{O}(35)$ & 119.2 \\
\hline $\mathrm{O}(108)-\mathrm{K}(4)-\mathrm{O}(105)$ & 92.6 & $\mathrm{O}(34)-\mathrm{K}(5)-\mathrm{O}(35)$ & 60.8 \\
\hline $\mathrm{O}(102)-\mathrm{K}(4)-\mathrm{O}(105)$ & 157.6 & $\mathrm{O}(36) \# 2-\mathrm{K}(5)-\mathrm{O}(35)$ & 120.8 \\
\hline $\mathrm{O}(108)-\mathrm{K}(4)-\mathrm{O}(101)$ & 123.9 & $\mathrm{O}(36)-\mathrm{K}(5)-\mathrm{O}(35)$ & 59.2 \\
\hline $\mathrm{O}(102)-\mathrm{K}(4)-\mathrm{O}(101)$ & 58.8 & $\mathrm{O}(35) \# 2-\mathrm{K}(5)-\mathrm{O}(35)$ & 180.0 \\
\hline $\mathrm{O}(105)-\mathrm{K}(4)-\mathrm{O}(101)$ & 118.4 & $\mathrm{O}(34) \# 2-\mathrm{K}(5)-\mathrm{C}(176) \# 2$ & 80.1 \\
\hline $\mathrm{O}(108)-\mathrm{K}(4)-\mathrm{O}(106)$ & 125.1 & $\mathrm{O}(34)-\mathrm{K}(5)-\mathrm{C}(176) \# 2$ & 99.9 \\
\hline $\mathrm{O}(102)-\mathrm{K}(4)-\mathrm{O}(106)$ & 114.2 & $\mathrm{O}(36) \# 2-\mathrm{K}(5)-\mathrm{C}(176) \# 2$ & 101.7 \\
\hline $\mathrm{O}(105)-\mathrm{K}(4)-\mathrm{O}(106)$ & 58.6 & $\mathrm{O}(36)-\mathrm{K}(5)-\mathrm{C}(176) \# 2$ & 78.3 \\
\hline $\mathrm{O}(101)-\mathrm{K}(4)-\mathrm{O}(106)$ & 59.8 & $\mathrm{O}(35) \# 2-\mathrm{K}(5)-\mathrm{C}(176) \# 2$ & 103.4 \\
\hline $\mathrm{O}(108)-\mathrm{K}(4)-\mathrm{O}(103)$ & 80.4 & $\mathrm{O}(35)-\mathrm{K}(5)-\mathrm{C}(176) \# 2$ & 76.6 \\
\hline $\mathrm{O}(102)-\mathrm{K}(4)-\mathrm{O}(103)$ & 59.1 & $\mathrm{O}(34) \# 2-\mathrm{K}(5)-\mathrm{C}(177) \# 2$ & 71.8 \\
\hline $\mathrm{O}(105)-\mathrm{K}(4)-\mathrm{O}(103)$ & 116.1 & $\mathrm{O}(34)-\mathrm{K}(5)-\mathrm{C}(177) \# 2$ & 108.2 \\
\hline $\mathrm{O}(101)-\mathrm{K}(4)-\mathrm{O}(103)$ & 117.5 & $\mathrm{O}(36) \# 2-\mathrm{K}(5)-\mathrm{C}(177) \# 2$ & 86.2 \\
\hline $\mathrm{O}(106)-\mathrm{K}(4)-\mathrm{O}(103)$ & 152.5 & $\mathrm{O}(36)-\mathrm{K}(5)-\mathrm{C}(177) \# 2$ & 93.8 \\
\hline $\mathrm{O}(108)-\mathrm{K}(4)-\mathrm{O}(104)$ & 83.4 & $\mathrm{O}(35) \# 2-\mathrm{K}(5)-\mathrm{C}(177) \# 2$ & 80.1 \\
\hline $\mathrm{O}(102)-\mathrm{K}(4)-\mathrm{O}(104)$ & 112.8 & $\mathrm{O}(35)-\mathrm{K}(5)-\mathrm{C}(177) \# 2$ & 99.9 \\
\hline $\mathrm{O}(105)-\mathrm{K}(4)-\mathrm{O}(104)$ & 58.0 & $\mathrm{C}(176) \# 2-\mathrm{K}(5)-\mathrm{C}(177) \# 2$ & 23.8 \\
\hline $\mathrm{O}(101)-\mathrm{K}(4)-\mathrm{O}(104)$ & 152.3 & $\mathrm{C}(1)-\mathrm{N}(1)-\mathrm{C}(2)$ & 107.9 \\
\hline $\mathrm{O}(106)-\mathrm{K}(4)-\mathrm{O}(104)$ & 110.2 & $\mathrm{C}(1)-\mathrm{N}(1)-\mathrm{Zr}(1)$ & 142.6 \\
\hline $\mathrm{O}(103)-\mathrm{K}(4)-\mathrm{O}(104)$ & 58.1 & $\mathrm{C}(2)-\mathrm{N}(1)-\mathrm{Zr}(1)$ & 109.4 \\
\hline $\mathrm{O}(108)-\mathrm{K}(4)-\mathrm{O}(107)$ & 57.5 & $\mathrm{C}(3)-\mathrm{N}(2)-\mathrm{C}(4)$ & 112.7 \\
\hline $\mathrm{O}(102)-\mathrm{K}(4)-\mathrm{O}(107)$ & 111.0 & $\mathrm{C}(3)-\mathrm{N}(2)-\operatorname{Zr}(1)$ & 111.4 \\
\hline $\mathrm{O}(105)-\mathrm{K}(4)-\mathrm{O}(107)$ & 88.3 & $\mathrm{C}(4)-\mathrm{N}(2)-\mathrm{Zr}(1)$ & 134.8 \\
\hline $\mathrm{O}(101)-\mathrm{K}(4)-\mathrm{O}(107)$ & 76.8 & $\mathrm{C}(5)-\mathrm{N}(3)-\mathrm{C}(6)$ & 108.8 \\
\hline $\mathrm{O}(106)-\mathrm{K}(4)-\mathrm{O}(107)$ & 74.6 & $\mathrm{C}(5)-\mathrm{N}(3)-\mathrm{Zr}(1)$ & 132.5 \\
\hline
\end{tabular}




\begin{tabular}{|c|c|c|c|}
\hline $\mathrm{C}(6)-\mathrm{N}(3)-\mathrm{Zr}(1)$ & 118.2 & $\mathrm{C}(124)-\mathrm{O}(19)-\mathrm{C}(123)$ & 116.1 \\
\hline $\mathrm{C}(21)-\mathrm{N}(4)-\mathrm{C}(22)$ & 108.0 & $\mathrm{C}(124)-\mathrm{O}(19)-\mathrm{K}(3)$ & 113.5 \\
\hline $\mathrm{C}(21)-\mathrm{N}(4)-\mathrm{Zr}(2)$ & 141.3 & $\mathrm{C}(123)-\mathrm{O}(19)-\mathrm{K}(3)$ & 113.4 \\
\hline $\mathrm{C}(22)-\mathrm{N}(4)-\mathrm{Zr}(2)$ & 110.6 & $\mathrm{C}(125)-\mathrm{O}(20)-\mathrm{C}(126)$ & 115.0 \\
\hline $\mathrm{C}(23)-\mathrm{N}(5)-\mathrm{C}(24)$ & 112.8 & $\mathrm{C}(125)-\mathrm{O}(20)-\mathrm{K}(3)$ & 116.5 \\
\hline $\mathrm{C}(23)-\mathrm{N}(5)-\mathrm{Zr}(2)$ & 128.8 & $\mathrm{C}(126)-\mathrm{O}(20)-\mathrm{K}(3)$ & 113.2 \\
\hline $\mathrm{C}(24)-\mathrm{N}(5)-\mathrm{Zr}(2)$ & 118.0 & $\mathrm{C}(127)-\mathrm{O}(21)-\mathrm{C}(128)$ & 112.1 \\
\hline$C(25)-N(6)-C(26)$ & 112.8 & $\mathrm{C}(127)-\mathrm{O}(21)-\mathrm{K}(3)$ & 115.4 \\
\hline $\mathrm{C}(25)-\mathrm{N}(6)-\mathrm{Zr}(2)$ & 110.6 & $\mathrm{C}(128)-\mathrm{O}(21)-\mathrm{K}(3)$ & 114.8 \\
\hline $\mathrm{C}(26)-\mathrm{N}(6)-\mathrm{Zr}(2)$ & 135.5 & $\mathrm{C}(144)-\mathrm{O}(22)-\mathrm{C}(154)$ & 109.5 \\
\hline $\mathrm{C}(41)-\mathrm{N}(7)-\mathrm{C}(42)$ & 109.1 & $\mathrm{C}(144)-\mathrm{O}(22)-\mathrm{K}(2)$ & 117.2 \\
\hline $\mathrm{C}(41)-\mathrm{N}(7)-\mathrm{Zr}(3)$ & 142.3 & $\mathrm{C}(154)-\mathrm{O}(22)-\mathrm{K}(2)$ & 113.1 \\
\hline C(42)-N(7)-Zr(3) & 108.5 & $\mathrm{C}(146)-\mathrm{O}(23)-\mathrm{C}(145)$ & 115.2 \\
\hline $\mathrm{C}(44)-\mathrm{N}(8)-\mathrm{C}(43)$ & 110.6 & $\mathrm{C}(146)-\mathrm{O}(23)-\mathrm{K}(2)$ & 114.4 \\
\hline $\mathrm{C}(44)-\mathrm{N}(8)-\mathrm{Zr}(3)$ & 134.6 & $\mathrm{C}(145)-\mathrm{O}(23)-\mathrm{K}(2)$ & 115.5 \\
\hline $\mathrm{C}(43)-\mathrm{N}(8)-\mathrm{Zr}(3)$ & 114.5 & $\mathrm{C}(148)-\mathrm{O}(24)-\mathrm{C}(147)$ & 112.8 \\
\hline $\mathrm{C}(46)-\mathrm{N}(9)-\mathrm{C}(45)$ & 109.7 & $\mathrm{C}(148)-\mathrm{O}(24)-\mathrm{K}(2)$ & 119.7 \\
\hline $\mathrm{C}(46)-\mathrm{N}(9)-\mathrm{Zr}(3)$ & 120.3 & $\mathrm{C}(147)-\mathrm{O}(24)-\mathrm{K}(2)$ & 116.8 \\
\hline $\mathrm{C}(45)-\mathrm{N}(9)-\mathrm{Zr}(3)$ & 129.8 & $\mathrm{C}(150)-\mathrm{O}(25)-\mathrm{C}(149)$ & 109.2 \\
\hline $\mathrm{C}(62)-\mathrm{N}(10)-\mathrm{C}(61)$ & 106.7 & $\mathrm{C}(150)-\mathrm{O}(25)-\mathrm{K}(2)$ & 111.2 \\
\hline $\mathrm{C}(62)-\mathrm{N}(10)-\mathrm{Zr}(4)$ & 134.8 & $\mathrm{C}(149)-\mathrm{O}(25)-\mathrm{K}(2)$ & 114.9 \\
\hline $\mathrm{C}(61)-\mathrm{N}(10)-\mathrm{Zr}(4)$ & 118.5 & $\mathrm{C}(99)-\mathrm{O}(26)-\mathrm{C}(151)$ & 111.6 \\
\hline $\mathrm{C}(64)-\mathrm{N}(11)-\mathrm{C}(63)$ & 106.6 & $\mathrm{C}(99)-\mathrm{O}(26)-\mathrm{K}(2)$ & 118.8 \\
\hline C(64)-N(11)-Zr(4) & 128.1 & $\mathrm{C}(151)-\mathrm{O}(26)-\mathrm{K}(2)$ & 119.1 \\
\hline C(63)-N(11)-Zr(4) & 125.0 & $\mathrm{C}(153)-\mathrm{O}(27)-\mathrm{C}(152)$ & 107.1 \\
\hline $\mathrm{C}(66)-\mathrm{N}(12)-\mathrm{C}(65)$ & 110.9 & $\mathrm{C}(153)-\mathrm{O}(27)-\mathrm{K}(2)$ & 116.7 \\
\hline $\mathrm{C}(66)-\mathrm{N}(12)-\mathrm{Zr}(4)$ & 121.8 & $\mathrm{C}(152)-\mathrm{O}(27)-\mathrm{K}(2)$ & 118.4 \\
\hline $\mathrm{C}(65)-\mathrm{N}(12)-\mathrm{Zr}(4)$ & 126.8 & $\mathrm{C}(166)-\mathrm{O}(28)-\mathrm{C}(155)$ & 114.6 \\
\hline $\mathrm{C}(86) \# 1-\mathrm{O}(1)-\mathrm{C}(81)$ & 112.8 & $\mathrm{C}(166)-\mathrm{O}(28)-\mathrm{K}(2)$ & 125.9 \\
\hline C(86)\#1-O(1)-K(1) & 114.6 & $\mathrm{C}(155)-\mathrm{O}(28)-\mathrm{K}(2)$ & 115.8 \\
\hline $\mathrm{C}(81)-\mathrm{O}(1)-\mathrm{K}(1)$ & 114.9 & $\mathrm{C}(157)-\mathrm{O}(29)-\mathrm{C}(156)$ & 109.6 \\
\hline $\mathrm{C}(83)-\mathrm{O}(2)-\mathrm{C}(82)$ & 111.8 & $\mathrm{C}(158)-\mathrm{O}(30)-\mathrm{C}(159)$ & 112.1 \\
\hline $\mathrm{C}(83)-\mathrm{O}(2)-\mathrm{K}(1)$ & 115.0 & $\mathrm{C}(161)-\mathrm{O}(31)-\mathrm{C}(160)$ & 113.0 \\
\hline $\mathrm{C}(82)-\mathrm{O}(2)-\mathrm{K}(1)$ & 116.4 & $\mathrm{C}(163)-\mathrm{O}(32)-\mathrm{C}(162)$ & 111.8 \\
\hline $\mathrm{C}(85)-\mathrm{O}(3)-\mathrm{C}(84)$ & 111.4 & $\mathrm{C}(164)-\mathrm{O}(33)-\mathrm{C}(165)$ & 112.0 \\
\hline $\mathrm{C}(85)-\mathrm{O}(3)-\mathrm{K}(1)$ & 113.8 & $\mathrm{C}(164)-\mathrm{O}(33)-\mathrm{K}(2)$ & 115.0 \\
\hline $\mathrm{C}(84)-\mathrm{O}(3)-\mathrm{K}(1)$ & 114.8 & $\mathrm{C}(165)-\mathrm{O}(33)-\mathrm{K}(2)$ & 103.3 \\
\hline $\mathrm{C}(129)-\mathrm{O}(16)-\mathrm{C}(118)$ & 115.3 & $\mathrm{C}(168)-\mathrm{O}(34)-\mathrm{C}(169)$ & 111.4 \\
\hline $\mathrm{C}(129)-\mathrm{O}(16)-\mathrm{K}(3)$ & 113.4 & $\mathrm{C}(168)-\mathrm{O}(34)-\mathrm{K}(5)$ & 113.0 \\
\hline $\mathrm{C}(118)-\mathrm{O}(16)-\mathrm{K}(3)$ & 116.3 & $\mathrm{C}(169)-\mathrm{O}(34)-\mathrm{K}(5)$ & 116.5 \\
\hline $\mathrm{C}(119)-\mathrm{O}(17)-\mathrm{C}(120)$ & 113.7 & $\mathrm{C}(171)-\mathrm{O}(35)-\mathrm{C}(170)$ & 112.6 \\
\hline C(119)-O(17)-K(3) & 113.6 & $\mathrm{C}(171)-\mathrm{O}(35)-\mathrm{K}(5)$ & 116.0 \\
\hline $\mathrm{C}(120)-\mathrm{O}(17)-\mathrm{K}(3)$ & 114.1 & $\mathrm{C}(170)-\mathrm{O}(35)-\mathrm{K}(5)$ & 113.1 \\
\hline $\mathrm{C}(121)-\mathrm{O}(18)-\mathrm{C}(122)$ & 113.5 & $\mathrm{C}(167) \# 2-\mathrm{O}(36)-\mathrm{C}(172)$ & 113.0 \\
\hline $\mathrm{C}(121)-\mathrm{O}(18)-\mathrm{K}(3)$ & 116.0 & $\mathrm{C}(167) \# 2-\mathrm{O}(36)-\mathrm{K}(5)$ & 112.7 \\
\hline $\mathrm{C}(122)-\mathrm{O}(18)-\mathrm{K}(3)$ & 112.7 & $\mathrm{C}(172)-\mathrm{O}(36)-\mathrm{K}(5)$ & 115.9 \\
\hline
\end{tabular}




\begin{tabular}{|c|c|c|c|}
\hline $\mathrm{C}(228)-\mathrm{O}(101)-\mathrm{C}(239)$ & 117.7 & $\mathrm{C}(12)-\mathrm{Si}(4)-\mathrm{C}(11)$ & 105.3 \\
\hline $\mathrm{C}(228)-\mathrm{O}(101)-\mathrm{K}(4)$ & 118.2 & $\mathrm{C}(12)-\mathrm{Si}(4)-\mathrm{C}(10)$ & 105.4 \\
\hline $\mathrm{C}(239)-\mathrm{O}(101)-\mathrm{K}(4)$ & 113.4 & $C(11)-\operatorname{Si}(4)-C(10)$ & 106.5 \\
\hline $\mathrm{C}(230)-\mathrm{O}(102)-\mathrm{C}(229)$ & 111.1 & C(12)-Si(4)-Si(1) & 111.2 \\
\hline $\mathrm{C}(230)-\mathrm{O}(102)-\mathrm{K}(4)$ & 111.1 & $\mathrm{C}(11)-\mathrm{Si}(4)-\mathrm{Si}(1)$ & 111.5 \\
\hline $\mathrm{C}(229)-\mathrm{O}(102)-\mathrm{K}(4)$ & 111.3 & C(10)-Si(4)-Si(1) & 116.1 \\
\hline $\mathrm{C}(232)-\mathrm{O}(103)-\mathrm{C}(231)$ & 116.8 & $C(16)-\operatorname{Si}(5)-C(15)$ & 108.3 \\
\hline $\mathrm{C}(232)-\mathrm{O}(103)-\mathrm{K}(4)$ & 122.2 & $C(16)-\operatorname{Si}(5)-C(17)$ & 106.8 \\
\hline $\mathrm{C}(231)-\mathrm{O}(103)-\mathrm{K}(4)$ & 109.0 & $C(15)-\operatorname{Si}(5)-C(17)$ & 104.6 \\
\hline $\mathrm{C}(234)-\mathrm{O}(104)-\mathrm{C}(233)$ & 128.2 & $C(16)-\operatorname{Si}(5)-\operatorname{Si}(2)$ & 113.8 \\
\hline $\mathrm{C}(234)-\mathrm{O}(104)-\mathrm{K}(4)$ & 106.2 & $C(15)-\operatorname{Si}(5)-\operatorname{Si}(2)$ & 108.0 \\
\hline $\mathrm{C}(233)-\mathrm{O}(104)-\mathrm{K}(4)$ & 108.3 & $C(17)-\operatorname{Si}(5)-\operatorname{Si}(2)$ & 114.9 \\
\hline $\mathrm{C}(235)-\mathrm{O}(105)-\mathrm{C}(236)$ & 113.8 & $C(19)-\operatorname{Si}(6)-C(18)$ & 107.6 \\
\hline $\mathrm{C}(235)-\mathrm{O}(105)-\mathrm{K}(4)$ & 117.1 & $C(19)-\operatorname{Si}(6)-C(20)$ & 104.5 \\
\hline $\mathrm{C}(236)-\mathrm{O}(105)-\mathrm{K}(4)$ & 118.8 & $C(18)-\operatorname{Si}(6)-C(20)$ & 105.9 \\
\hline $\mathrm{C}(238)-\mathrm{O}(106)-\mathrm{C}(237)$ & 117.0 & C(19)-Si(6)-Si(2) & 111.6 \\
\hline $\mathrm{C}(238)-\mathrm{O}(106)-\mathrm{K}(4)$ & 109.2 & $\mathrm{C}(18)-\operatorname{Si}(6)-\operatorname{Si}(2)$ & 112.9 \\
\hline $\mathrm{C}(237)-\mathrm{O}(106)-\mathrm{K}(4)$ & 108.2 & $\mathrm{C}(20)-\mathrm{Si}(6)-\mathrm{Si}(2)$ & 113.7 \\
\hline $\mathrm{C}(249)-\mathrm{O}(107)-\mathrm{C}(241)$ & 111.1 & $\mathrm{C}(33)-\mathrm{Si}(7)-\mathrm{Si}(10)$ & 96.4 \\
\hline $\mathrm{C}(249)-\mathrm{O}(107)-\mathrm{K}(4)$ & 125.8 & $\mathrm{C}(33)-\mathrm{Si}(7)-\mathrm{Si}(9)$ & 103.5 \\
\hline $\mathrm{C}(241)-\mathrm{O}(107)-\mathrm{K}(4)$ & 95.6 & $\operatorname{Si}(10)-\operatorname{Si}(7)-\operatorname{Si}(9)$ & 105.4 \\
\hline $\mathrm{C}(240)-\mathrm{O}(108)-\mathrm{C}(247)$ & 114.2 & C(33)-Si(7)-Zr(2) & 113.6 \\
\hline $\mathrm{C}(240)-\mathrm{O}(108)-\mathrm{K}(4)$ & 123.8 & $\operatorname{Si}(10)-\operatorname{Si}(7)-\operatorname{Zr}(2)$ & 124.2 \\
\hline $\mathrm{C}(247)-\mathrm{O}(108)-\mathrm{K}(4)$ & 121.8 & $\operatorname{Si}(9)-\operatorname{Si}(7)-\operatorname{Zr}(2)$ & 111.1 \\
\hline $\mathrm{C}(248)-\mathrm{O}(112)-\mathrm{C}(251)$ & 112.8 & $\mathrm{C}(34)-\mathrm{Si}(8)-\mathrm{Si}(11)$ & 99.8 \\
\hline $\mathrm{C}(250)-\mathrm{O}(113)-\mathrm{C}(253)$ & 110.1 & C(34)-Si(8)-Si(12) & 104.8 \\
\hline $\mathrm{C}(255)-\mathrm{O}(114)-\mathrm{C}(254)$ & 104.3 & $\mathrm{Si}(11)-\mathrm{Si}(8)-\mathrm{Si}(12)$ & 103.8 \\
\hline $\mathrm{C}(252)-\mathrm{O}(117)-\mathrm{C}(259)$ & 113.6 & $\mathrm{C}(34)-\mathrm{Si}(8)-\operatorname{Zr}(2)$ & 114.9 \\
\hline $\mathrm{C}(13)-\mathrm{Si}(1)-\mathrm{Si}(4)$ & 101.5 & $\operatorname{Si}(11)-\operatorname{Si}(8)-\operatorname{Zr}(2)$ & 118.6 \\
\hline C(13)-Si(1)-Si(3) & 99.4 & $\operatorname{Si}(12)-\operatorname{Si}(8)-\operatorname{Zr}(2)$ & 113.1 \\
\hline $\operatorname{Si}(4)-\operatorname{Si}(1)-\operatorname{Si}(3)$ & 104.8 & $\mathrm{C}(27)-\mathrm{Si}(9)-\mathrm{C}(28)$ & 108.6 \\
\hline C(13)-Si(1)-Zr(1) & 113.2 & $\mathrm{C}(27)-\mathrm{Si}(9)-\mathrm{C}(29)$ & 103.5 \\
\hline $\operatorname{Si}(4)-\operatorname{Si}(1)-\operatorname{Zr}(1)$ & 118.2 & $\mathrm{C}(28)-\mathrm{Si}(9)-\mathrm{C}(29)$ & 105.3 \\
\hline $\operatorname{Si}(3)-\operatorname{Si}(1)-Z r(1)$ & 117.1 & $\mathrm{C}(27)-\mathrm{Si}(9)-\mathrm{Si}(7)$ & 111.3 \\
\hline$C(14)-\operatorname{Si}(2)-\operatorname{Si}(5)$ & 101.5 & $\mathrm{C}(28)-\mathrm{Si}(9)-\mathrm{Si}(7)$ & 111.3 \\
\hline$C(14)-\operatorname{Si}(2)-\operatorname{Si}(6)$ & 102.3 & C(29)-Si(9)-Si(7) & 116.2 \\
\hline $\operatorname{Si}(5)-\operatorname{Si}(2)-\operatorname{Si}(6)$ & 105.3 & $\mathrm{C}(30)-\mathrm{Si}(10)-\mathrm{C}(32)$ & 108.3 \\
\hline$C(14)-\operatorname{Si}(2)-\operatorname{Zr}(1)$ & 114.8 & $\mathrm{C}(30)-\mathrm{Si}(10)-\mathrm{C}(31)$ & 105.5 \\
\hline $\operatorname{Si}(5)-\operatorname{Si}(2)-\operatorname{Zr}(1)$ & 116.7 & $\mathrm{C}(32)-\mathrm{Si}(10)-\mathrm{C}(31)$ & 102.9 \\
\hline $\operatorname{Si}(6)-\operatorname{Si}(2)-\operatorname{Zr}(1)$ & 114.4 & C(30)-Si(10)-Si(7) & 111.0 \\
\hline C(7)-Si(3)-C(8) & 105.7 & $C(32)-\operatorname{Si}(10)-\operatorname{Si}(7)$ & 118.9 \\
\hline$C(7)-S i(3)-C(9)$ & 104.6 & C(31)-Si(10)-Si(7) & 109.2 \\
\hline C(8)-Si(3)-C(9) & 106.1 & $\mathrm{C}(35)-\mathrm{Si}(11)-\mathrm{C}(37)$ & 106.7 \\
\hline $\mathrm{C}(7)-\mathrm{Si}(3)-\mathrm{Si}(1)$ & 110.6 & $C(35)-\operatorname{Si}(11)-C(36)$ & 105.5 \\
\hline $\mathrm{C}(8)-\mathrm{Si}(3)-\mathrm{Si}(1)$ & 115.9 & $\mathrm{C}(37)-\mathrm{Si}(11)-\mathrm{C}(36)$ & 105.4 \\
\hline $\mathrm{C}(9)-\mathrm{Si}(3)-\mathrm{Si}(1)$ & 113.1 & $\mathrm{C}(35)-\mathrm{Si}(11)-\mathrm{Si}(8)$ & 110.9 \\
\hline
\end{tabular}




\begin{tabular}{|c|c|c|c|}
\hline C(37)-Si(11)-Si(8) & 116.0 & $\operatorname{Si}(21)-\operatorname{Si}(19)-\operatorname{Si}(20)$ & 102.5 \\
\hline C(36)-Si(11)-Si(8) & 111.6 & C(73)-Si(19)-Zr(4) & 112.4 \\
\hline $\mathrm{C}(40)-\mathrm{Si}(12)-\mathrm{C}(39)$ & 107.3 & $\operatorname{Si}(21)-\operatorname{Si}(19)-\operatorname{Zr}(4)$ & 122.3 \\
\hline $\mathrm{C}(40)-\mathrm{Si}(12)-\mathrm{C}(38)$ & 105.9 & $\operatorname{Si}(20)-\operatorname{Si}(19)-Z r(4)$ & 114.5 \\
\hline C(39)-Si(12)-C(38) & 104.6 & $C(68)-\operatorname{Si}(20)-C(67)$ & 103.6 \\
\hline C(40)-Si(12)-Si(8) & 111.9 & $\mathrm{C}(68)-\mathrm{Si}(20)-\mathrm{C}(69)$ & 105.9 \\
\hline $\mathrm{C}(39)-\mathrm{Si}(12)-\mathrm{Si}(8)$ & 110.5 & $C(67)-\operatorname{Si}(20)-C(69)$ & 107.1 \\
\hline C(38)-Si(12)-Si(8) & 116.0 & $\mathrm{C}(68)-\mathrm{Si}(20)-\mathrm{Si}(19)$ & 112.2 \\
\hline C(60)-Si(13)-Si(14) & 98.3 & $C(67)-\operatorname{Si}(20)-\operatorname{Si}(19)$ & 113.8 \\
\hline C(60)-Si(13)-Si(15) & 101.9 & C(69)-Si(20)-Si(19) & 113.4 \\
\hline $\operatorname{Si}(14)-\operatorname{Si}(13)-\operatorname{Si}(15)$ & 106.2 & $\mathrm{C}(71)-\mathrm{Si}(21)-\mathrm{C}(70)$ & 105.4 \\
\hline $\mathrm{C}(60)-\operatorname{Si}(13)-\operatorname{Zr}(3)$ & 114.3 & $\mathrm{C}(71)-\mathrm{Si}(21)-\mathrm{C}(72)$ & 107.6 \\
\hline $\operatorname{Si}(14)-\operatorname{Si}(13)-\operatorname{Zr}(3)$ & 118.1 & $\mathrm{C}(70)-\mathrm{Si}(21)-\mathrm{C}(72)$ & 104.7 \\
\hline $\operatorname{Si}(15)-\operatorname{Si}(13)-\operatorname{Zr}(3)$ & 115.6 & $\mathrm{C}(71)-\mathrm{Si}(21)-\mathrm{Si}(19)$ & 114.6 \\
\hline$C(49)-\operatorname{Si}(14)-C(47)$ & 105.3 & $\mathrm{C}(70)-\mathrm{Si}(21)-\mathrm{Si}(19)$ & 115.8 \\
\hline C(49)-Si(14)-C(48) & 107.4 & C(72)-Si(21)-Si(19) & 108.1 \\
\hline C(47)-Si(14)-C(48) & 105.2 & $C(74)-\operatorname{Si}(22)-\operatorname{Si}(23)$ & 100.1 \\
\hline C(49)-Si(14)-Si(13) & 113.3 & $\mathrm{C}(74)-\mathrm{Si}(22)-\mathrm{Si}(24)$ & 102.1 \\
\hline $\mathrm{C}(47)-\mathrm{Si}(14)-\mathrm{Si}(13)$ & 110.6 & $\operatorname{Si}(23)-\operatorname{Si}(22)-\operatorname{Si}(24)$ & 104.5 \\
\hline C(48)-Si(14)-Si(13) & 114.2 & $\mathrm{C}(74)-\mathrm{Si}(22)-\mathrm{Zr}(4)$ & 114.5 \\
\hline $\mathrm{C}(52)-\mathrm{Si}(15)-\mathrm{C}(51)$ & 106.0 & $\operatorname{Si}(23)-\operatorname{Si}(22)-Z r(4)$ & 121.9 \\
\hline $\mathrm{C}(52)-\mathrm{Si}(15)-\mathrm{C}(50)$ & 105.5 & $\operatorname{Si}(24)-\operatorname{Si}(22)-\operatorname{Zr}(4)$ & 111.4 \\
\hline$C(51)-\operatorname{Si}(15)-C(50)$ & 106.4 & $\mathrm{C}(77)-\mathrm{Si}(23)-\mathrm{C}(75)$ & 105.9 \\
\hline $\mathrm{C}(52)-\mathrm{Si}(15)-\mathrm{Si}(13)$ & 111.3 & $\mathrm{C}(77)-\mathrm{Si}(23)-\mathrm{C}(76)$ & 105.9 \\
\hline $\mathrm{C}(51)-\mathrm{Si}(15)-\mathrm{Si}(13)$ & 110.7 & $\mathrm{C}(75)-\mathrm{Si}(23)-\mathrm{C}(76)$ & 105.3 \\
\hline $\mathrm{C}(50)-\mathrm{Si}(15)-\mathrm{Si}(13)$ & 116.4 & $\mathrm{C}(77)-\mathrm{Si}(23)-\mathrm{Si}(22)$ & 117.7 \\
\hline C(59)-Si(16)-Si(17) & 103.0 & $\mathrm{C}(75)-\mathrm{Si}(23)-\mathrm{Si}(22)$ & 110.5 \\
\hline C(59)-Si(16)-Si(18) & 101.5 & $\mathrm{C}(76)-\mathrm{Si}(23)-\mathrm{Si}(22)$ & 110.8 \\
\hline $\operatorname{Si}(17)-\operatorname{Si}(16)-\operatorname{Si}(18)$ & 105.7 & $\mathrm{C}(79)-\mathrm{Si}(24)-\mathrm{C}(78)$ & 104.8 \\
\hline C(59)-Si(16)-Zr(3) & 114.0 & $\mathrm{C}(79)-\mathrm{Si}(24)-\mathrm{C}(80)$ & 105.7 \\
\hline $\operatorname{Si}(17)-\operatorname{Si}(16)-\operatorname{Zr}(3)$ & 117.5 & $\mathrm{C}(78)-\mathrm{Si}(24)-\mathrm{C}(80)$ & 104.9 \\
\hline $\operatorname{Si}(18)-\operatorname{Si}(16)-\operatorname{Zr}(3)$ & 113.3 & C(79)-Si(24)-Si(22) & 111.3 \\
\hline $\mathrm{C}(55)-\mathrm{Si}(17)-\mathrm{C}(53)$ & 107.4 & $\mathrm{C}(78)-\mathrm{Si}(24)-\mathrm{Si}(22)$ & 114.5 \\
\hline $\mathrm{C}(55)-\mathrm{Si}(17)-\mathrm{C}(54)$ & 104.8 & $\mathrm{C}(80)-\mathrm{Si}(24)-\mathrm{Si}(22)$ & 114.7 \\
\hline C(53)-Si(17)-C(54) & 107.7 & $\mathrm{~N}(2)-\mathrm{Zr}(1)-\mathrm{N}(1)$ & 98.8 \\
\hline C(55)-Si(17)-Si(16) & 115.1 & $\mathrm{~N}(2)-\mathrm{Zr}(1)-\mathrm{N}(3)$ & 110.5 \\
\hline $\mathrm{C}(53)-\mathrm{Si}(17)-\mathrm{Si}(16)$ & 108.1 & $\mathrm{~N}(1)-\mathrm{Zr}(1)-\mathrm{N}(3)$ & 97.5 \\
\hline $\mathrm{C}(54)-\mathrm{Si}(17)-\mathrm{Si}(16)$ & 113.4 & $\mathrm{~N}(2)-\mathrm{Zr}(1)-\mathrm{Si}(2)$ & 97.1 \\
\hline $\mathrm{C}(58)-\mathrm{Si}(18)-\mathrm{C}(56)$ & 106.3 & $\mathrm{~N}(1)-\mathrm{Zr}(1)-\mathrm{Si}(2)$ & 152.9 \\
\hline $\mathrm{C}(58)-\mathrm{Si}(18)-\mathrm{C}(57)$ & 105.2 & $\mathrm{~N}(3)-\mathrm{Zr}(1)-\mathrm{Si}(2)$ & 97.2 \\
\hline C(56)-Si(18)-C(57) & 105.9 & $\mathrm{~N}(2)-\mathrm{Zr}(1)-\mathrm{Si}(1)$ & 126.3 \\
\hline $\mathrm{C}(58)-\mathrm{Si}(18)-\mathrm{Si}(16)$ & 115.0 & $\mathrm{~N}(1)-\operatorname{Zr}(1)-\mathrm{Si}(1)$ & 83.5 \\
\hline C(56)-Si(18)-Si(16) & 112.2 & $\mathrm{~N}(3)-\operatorname{Zr}(1)-\mathrm{Si}(1)$ & 122.5 \\
\hline $\mathrm{C}(57)-\mathrm{Si}(18)-\mathrm{Si}(16)$ & 111.5 & $\mathrm{Si}(2)-\operatorname{Zr}(1)-\mathrm{Si}(1)$ & 69.4 \\
\hline C(73)-Si(19)-Si(21) & 99.2 & $\mathrm{~N}(6)-\mathrm{Zr}(2)-\mathrm{N}(5)$ & 103.9 \\
\hline C(73)-Si(19)-Si(20) & 103.3 & $\mathrm{~N}(6)-\operatorname{Zr}(2)-\mathrm{N}(4)$ & 104.7 \\
\hline
\end{tabular}




\begin{tabular}{|c|c|}
\hline $\mathrm{N}(5)-\operatorname{Zr}(2)-\mathrm{N}(4)$ & 95.2 \\
\hline $\mathrm{N}(6)-\operatorname{Zr}(2)-\operatorname{Si}(8)$ & 98.4 \\
\hline$N(5)-Z r(2)-\operatorname{Si}(8)$ & 95.5 \\
\hline$N(4)-\operatorname{Zr}(2)-\operatorname{Si}(8)$ & 151.4 \\
\hline$N(6)-Z r(2)-\operatorname{Si}(7)$ & 122.7 \\
\hline $\mathrm{N}(5)-\operatorname{Zr}(2)-\operatorname{Si}(7)$ & 132.2 \\
\hline $\mathrm{N}(4)-\operatorname{Zr}(2)-\operatorname{Si}(7)$ & 83.7 \\
\hline $\operatorname{Si}(8)-\operatorname{Zr}(2)-\operatorname{Si}(7)$ & 69.4 \\
\hline $\mathrm{N}(8)-\mathrm{Zr}(3)-\mathrm{N}(7)$ & 99.1 \\
\hline $\mathrm{N}(8)-\mathrm{Zr}(3)-\mathrm{N}(9)$ & 110.2 \\
\hline $\mathrm{N}(7)-\mathrm{Zr}(3)-\mathrm{N}(9)$ & 98.4 \\
\hline $\mathrm{N}(8)-\operatorname{Zr}(3)-\operatorname{Si}(16)$ & 97.0 \\
\hline $\mathrm{N}(7)-\mathrm{Zr}(3)-\mathrm{Si}(16)$ & 153.3 \\
\hline $\mathrm{N}(9)-\mathrm{Zr}(3)-\operatorname{Si}(16)$ & 95.9 \\
\hline $\mathrm{N}(8)-\operatorname{Zr}(3)-\operatorname{Si}(13)$ & 124.1 \\
\hline$N(7)-Z r(3)-\operatorname{Si}(13)$ & 84.2 \\
\hline N(9)-Zr(3)-Si(13) & 124.6 \\
\hline $\operatorname{Si}(16)-\operatorname{Zr}(3)-\operatorname{Si}(13)$ & 69.1 \\
\hline $\mathrm{N}(12)-\mathrm{Zr}(4)-\mathrm{N}(10)$ & 107.9 \\
\hline $\mathrm{N}(12)-\mathrm{Zr}(4)-\mathrm{N}(11)$ & 102.0 \\
\hline $\mathrm{N}(10)-\mathrm{Zr}(4)-\mathrm{N}(11)$ & 97.1 \\
\hline $\mathrm{N}(12)-\mathrm{Zr}(4)-\operatorname{Si}(22)$ & 104.9 \\
\hline $\mathrm{N}(10)-\operatorname{Zr}(4)-\operatorname{Si}(22)$ & 143.9 \\
\hline $\mathrm{N}(11)-\operatorname{Zr}(4)-\operatorname{Si}(22)$ & 90.3 \\
\hline $\mathrm{N}(12)-\operatorname{Zr}(4)-\operatorname{Si}(19)$ & 106.2 \\
\hline $\mathrm{N}(10)-\mathrm{Zr}(4)-\mathrm{Si}(19)$ & 87.5 \\
\hline $\mathrm{N}(11)-\mathrm{Zr}(4)-\mathrm{Si}(19)$ & 148.4 \\
\hline $\operatorname{Si}(22)-\operatorname{Zr}(4)-\operatorname{Si}(19)$ & 69.1 \\
\hline
\end{tabular}

Symmetry transformations used to generate equivalent atoms:

$\# 1-x+2,-y+1,-z+2 \quad \# 2-x,-y,-z$ 
Table S14. Anisotropic displacement parameters $\left(\AA^{2} \times 10^{3}\right)$ for 3 . The anisotropic displacement factor exponent takes the form: $-2 \pi^{2}\left(\mathrm{~h}^{2} \mathrm{a}^{* 2} \mathrm{U}^{11}+\ldots+2 \mathrm{hka} \mathrm{b}^{*} \mathrm{U}^{12}\right)$

\begin{tabular}{|c|c|c|c|c|c|c|}
\hline & $\mathrm{U}^{11}$ & $\mathrm{U}^{22}$ & $\mathrm{U}^{33}$ & $\mathrm{U}^{23}$ & $\mathrm{U}^{13}$ & $\mathrm{U}^{12}$ \\
\hline $\mathrm{C}(1)$ & 62 & 62 & 62 & 12 & 5 & 12 \\
\hline $\mathrm{C}(2)$ & 82 & 82 & 82 & 17 & 6 & 17 \\
\hline $\mathrm{C}(3)$ & 75 & 75 & 75 & 15 & 6 & 15 \\
\hline C(4) & 78 & 78 & 78 & 16 & 6 & 16 \\
\hline$C(5)$ & 81 & 81 & 81 & 16 & 6 & 16 \\
\hline$C(6)$ & 58 & 58 & 58 & 12 & 4 & 12 \\
\hline$C(7)$ & 47 & 47 & 47 & 10 & 4 & 10 \\
\hline C(8) & 51 & 51 & 51 & 10 & 4 & 10 \\
\hline C(9) & 50 & 50 & 50 & 10 & 4 & 10 \\
\hline $\mathrm{C}(10)$ & 56 & 56 & 56 & 11 & 4 & 11 \\
\hline $\mathrm{C}(11)$ & 77 & 77 & 77 & 15 & 6 & 15 \\
\hline $\mathrm{C}(12)$ & 55 & 55 & 55 & 11 & 4 & 11 \\
\hline $\mathrm{C}(13)$ & 35 & 35 & 35 & 7 & 3 & 7 \\
\hline $\mathrm{C}(14)$ & 36 & 36 & 36 & 7 & 3 & 7 \\
\hline $\mathrm{C}(15)$ & 71 & 71 & 71 & 14 & 5 & 14 \\
\hline$C(16)$ & 67 & 67 & 67 & 14 & 5 & 14 \\
\hline $\mathrm{C}(17)$ & 61 & 61 & 61 & 12 & 5 & 12 \\
\hline$C(18)$ & 51 & 51 & 51 & 10 & 4 & 10 \\
\hline$C(19)$ & 58 & 58 & 58 & 12 & 4 & 12 \\
\hline$C(20)$ & 54 & 54 & 54 & 11 & 4 & 11 \\
\hline $\mathrm{C}(21)$ & 57 & 57 & 57 & 12 & 4 & 12 \\
\hline $\mathrm{C}(22)$ & 57 & 57 & 57 & 12 & 4 & 12 \\
\hline $\mathrm{C}(23)$ & 62 & 62 & 62 & 13 & 5 & 13 \\
\hline $\mathrm{C}(24)$ & 55 & 55 & 55 & 11 & 4 & 11 \\
\hline $\mathrm{C}(25)$ & 48 & 48 & 48 & 10 & 4 & 10 \\
\hline$C(26)$ & 46 & 46 & 46 & 9 & 3 & 9 \\
\hline $\mathrm{C}(27)$ & 85 & 85 & 85 & 17 & 6 & 17 \\
\hline$C(28)$ & 76 & 76 & 76 & 15 & 6 & 15 \\
\hline$C(29)$ & 71 & 71 & 71 & 14 & 5 & 14 \\
\hline $\mathrm{C}(30)$ & 54 & 54 & 54 & 11 & 4 & 11 \\
\hline $\mathrm{C}(31)$ & 51 & 51 & 51 & 10 & 4 & 10 \\
\hline $\mathrm{C}(32)$ & 67 & 67 & 67 & 14 & 5 & 14 \\
\hline $\mathrm{C}(33)$ & 30 & 30 & 30 & 6 & 2 & 6 \\
\hline $\mathrm{C}(34)$ & 38 & 38 & 38 & 8 & 3 & 8 \\
\hline $\mathrm{C}(35)$ & 55 & 55 & 55 & 11 & 4 & 11 \\
\hline $\mathrm{C}(36)$ & 57 & 57 & 57 & 11 & 4 & 11 \\
\hline $\mathrm{C}(37)$ & 59 & 59 & 59 & 12 & 4 & 12 \\
\hline $\mathrm{C}(38)$ & 41 & 41 & 41 & 8 & 3 & 8 \\
\hline $\mathrm{C}(39)$ & 42 & 42 & 42 & 9 & 3 & 9 \\
\hline $\mathrm{C}(40)$ & 40 & 40 & 40 & 8 & 3 & 8 \\
\hline
\end{tabular}




$\begin{array}{lcccccc}\mathrm{C}(41) & 51 & 51 & 51 & 10 & 4 & 10 \\ \mathrm{C}(42) & 70 & 70 & 70 & 14 & 5 & 14 \\ \mathrm{C}(43) & 78 & 78 & 78 & 16 & 6 & 16 \\ \mathrm{C}(44) & 69 & 69 & 69 & 14 & 5 & 14 \\ \mathrm{C}(45) & 67 & 67 & 67 & 13 & 5 & 13 \\ \mathrm{C}(46) & 47 & 47 & 47 & 9 & 3 & 9 \\ \mathrm{C}(47) & 43 & 43 & 43 & 9 & 3 & 9 \\ \mathrm{C}(48) & 50 & 50 & 50 & 10 & 4 & 10 \\ \mathrm{C}(49) & 48 & 48 & 48 & 10 & 4 & 10 \\ \mathrm{C}(50) & 52 & 52 & 52 & 10 & 4 & 10 \\ \mathrm{C}(51) & 54 & 54 & 54 & 11 & 4 & 11 \\ \mathrm{C}(52) & 50 & 50 & 50 & 10 & 4 & 10 \\ \mathrm{C}(53) & 57 & 57 & 57 & 12 & 4 & 12 \\ \mathrm{C}(54) & 57 & 57 & 57 & 12 & 4 & 12 \\ \mathrm{C}(55) & 48 & 48 & 48 & 10 & 4 & 10 \\ \mathrm{C}(56) & 45 & 45 & 45 & 9 & 3 & 9 \\ \mathrm{C}(57) & 50 & 50 & 50 & 10 & 4 & 10 \\ \mathrm{C}(58) & 52 & 52 & 52 & 10 & 4 & 10 \\ \mathrm{C}(59) & 28 & 28 & 28 & 6 & 2 & 6 \\ \mathrm{C}(60) & 29 & 29 & 29 & 6 & 2 & 6 \\ \mathrm{C}(61) & 131 & 131 & 131 & 27 & 10 & 26 \\ \mathrm{C}(62) & 149 & 149 & 149 & 30 & 11 & 30 \\ \mathrm{C}(63) & 118 & 118 & 118 & 24 & 9 & 24 \\ \mathrm{C}(64) & 68 & 68 & 68 & 14 & 5 & 14 \\ \mathrm{C}(65) & 80 & 80 & 80 & 16 & 6 & 16 \\ \mathrm{C}(66) & 62 & 62 & 62 & 13 & 5 & 13 \\ \mathrm{C}(67) & 68 & 68 & 68 & 14 & 5 & 14 \\ \mathrm{C}(68) & 64 & 64 & 64 & 13 & 5 & 13 \\ \mathrm{C}(69) & 62 & 62 & 62 & 13 & 5 & 13 \\ \mathrm{C}(70) & 54 & 54 & 54 & 11 & 4 & 11 \\ \mathrm{C}(71) & 66 & 66 & 66 & 13 & 5 & 13 \\ \mathrm{C}(72) & 59 & 59 & 59 & 12 & 4 & 12 \\ \mathrm{C}(73) & 43 & 43 & 43 & 9 & 3 & 9 \\ \mathrm{C}(74) & 45 & 45 & 45 & 9 & 3 & 9 \\ \mathrm{C}(75) & 82 & 82 & 82 & 17 & 6 & 17 \\ \mathrm{C}(76) & 83 & 83 & 83 & 17 & 6 & 17 \\ \mathrm{C}(77) & 74 & 74 & 74 & 15 & 6 & 15 \\ \mathrm{C}(78) & 61 & 61 & 61 & 12 & 5 & 12 \\ \mathrm{C}(79) & 60 & 60 & 60 & 12 & 4 & 12 \\ \mathrm{C}(80) & 53 & 53 & 53 & 11 & 4 & 11 \\ \mathrm{C}(81) & 63 & 63 & 63 & 13 & 5 & 13 \\ \mathrm{C}(82) & 68 & 68 & 68 & 14 & 5 & 14 \\ \mathrm{C}(83) & 58 & 58 & 58 & 12 & 4 & 12 \\ \mathrm{C}(84) & 59 & 59 & 59 & 12 & 4 & 12 \\ \mathrm{C}(85) & 61 & 61 & 61 & 12 & 5 & 12 \\ & & & & & & \\ & & & & \mathrm{~s} 39 & & \end{array}$




$\begin{array}{lcccccc}\mathrm{C}(86) & 61 & 61 & 61 & 12 & 5 & 12 \\ \mathrm{C}(87) & 40 & 40 & 40 & 8 & 3 & 8 \\ \mathrm{C}(88) & 48 & 48 & 48 & 10 & 4 & 10 \\ \mathrm{C}(89) & 68 & 68 & 68 & 14 & 5 & 14 \\ \mathrm{C}(90) & 84 & 84 & 84 & 17 & 6 & 17 \\ \mathrm{C}(91) & 79 & 79 & 79 & 16 & 6 & 16 \\ \mathrm{C}(92) & 56 & 56 & 56 & 11 & 4 & 11 \\ \mathrm{C}(93) & 82 & 82 & 82 & 17 & 6 & 17 \\ \mathrm{C}(99) & 62 & 62 & 62 & 13 & 5 & 13 \\ \mathrm{C}(118) & 67 & 67 & 67 & 13 & 5 & 13 \\ \mathrm{C}(119) & 59 & 59 & 59 & 12 & 4 & 12 \\ \mathrm{C}(120) & 57 & 57 & 57 & 12 & 4 & 12 \\ \mathrm{C}(121) & 64 & 64 & 64 & 13 & 5 & 13 \\ \mathrm{C}(122) & 76 & 76 & 76 & 15 & 6 & 15 \\ \mathrm{C}(123) & 74 & 74 & 74 & 15 & 6 & 15 \\ \mathrm{C}(124) & 64 & 64 & 64 & 13 & 5 & 13 \\ \mathrm{C}(125) & 65 & 65 & 65 & 13 & 5 & 13 \\ \mathrm{C}(126) & 63 & 63 & 63 & 13 & 5 & 13 \\ \mathrm{C}(127) & 63 & 63 & 63 & 13 & 5 & 13 \\ \mathrm{C}(128) & 66 & 66 & 66 & 13 & 5 & 13 \\ \mathrm{C}(129) & 75 & 75 & 75 & 15 & 6 & 15 \\ \mathrm{C}(130) & 50 & 50 & 50 & 10 & 4 & 10 \\ \mathrm{C}(131) & 50 & 50 & 50 & 10 & 4 & 10 \\ \mathrm{C}(132) & 55 & 55 & 55 & 11 & 4 & 11 \\ \mathrm{C}(133) & 65 & 65 & 65 & 13 & 5 & 13 \\ \mathrm{C}(134) & 63 & 63 & 63 & 13 & 5 & 13 \\ \mathrm{C}(135) & 59 & 59 & 59 & 12 & 4 & 12 \\ \mathrm{C}(136) & 105 & 105 & 105 & 21 & 8 & 21 \\ \mathrm{C}(137) & 31 & 31 & 31 & 6 & 2 & 6 \\ \mathrm{C}(138) & 36 & 36 & 36 & 7 & 3 & 7 \\ \mathrm{C}(139) & 53 & 53 & 53 & 11 & 4 & 11 \\ \mathrm{C}(140) & 62 & 62 & 62 & 12 & 5 & 12 \\ \mathrm{C}(141) & 52 & 52 & 52 & 11 & 4 & 11 \\ \mathrm{C}(142) & 43 & 43 & 43 & 9 & 3 & 9 \\ \mathrm{C}(143) & 57 & 57 & 57 & 11 & 4 & 11 \\ \mathrm{C}(144) & 58 & 58 & 58 & 12 & 4 & 12 \\ \mathrm{C}(145) & 61 & 61 & 61 & 12 & 5 & 12 \\ \mathrm{C}(146) & 62 & 62 & 62 & 12 & 5 & 12 \\ \mathrm{C}(147) & 65 & 65 & 65 & 13 & 5 & 13 \\ \mathrm{C}(148) & 50 & 50 & 50 & 10 & 4 & 10 \\ \mathrm{C}(149) & 51 & 51 & 51 & 10 & 4 & 10 \\ \mathrm{C}(150) & 52 & 52 & 52 & 10 & 4 & 10 \\ \mathrm{C}(151) & 123 & 123 & 123 & 25 & 9 & 25 \\ \mathrm{C}(152) & 178 & 178 & 178 & 36 & 13 & 36 \\ \mathrm{C}(153) & 76 & 76 & 76 & 15 & 6 & 15 \\ & & & & & & \\ & & & & 540 & & \end{array}$




$\begin{array}{lcccccc}\mathrm{C}(154) & 61 & 61 & 61 & 12 & 5 & 12 \\ \mathrm{C}(155) & 45 & 45 & 45 & 9 & 3 & 9 \\ \mathrm{C}(156) & 39 & 39 & 39 & 8 & 3 & 8 \\ \mathrm{C}(157) & 49 & 49 & 49 & 10 & 4 & 10 \\ \mathrm{C}(158) & 51 & 51 & 51 & 10 & 4 & 10 \\ \mathrm{C}(159) & 63 & 63 & 63 & 13 & 5 & 13 \\ \mathrm{C}(160) & 58 & 58 & 58 & 12 & 4 & 12 \\ \mathrm{C}(161) & 58 & 58 & 58 & 12 & 4 & 12 \\ \mathrm{C}(162) & 54 & 54 & 54 & 11 & 4 & 11 \\ \mathrm{C}(163) & 50 & 50 & 50 & 10 & 4 & 10 \\ \mathrm{C}(164) & 46 & 46 & 46 & 9 & 3 & 9 \\ \mathrm{C}(165) & 41 & 41 & 41 & 8 & 3 & 8 \\ \mathrm{C}(166) & 38 & 38 & 38 & 8 & 3 & 8 \\ \mathrm{C}(167) & 49 & 49 & 49 & 10 & 4 & 10 \\ \mathrm{C}(168) & 58 & 58 & 58 & 12 & 4 & 12 \\ \mathrm{C}(169) & 55 & 55 & 55 & 11 & 4 & 11 \\ \mathrm{C}(170) & 55 & 55 & 55 & 11 & 4 & 11 \\ \mathrm{C}(171) & 55 & 55 & 55 & 11 & 4 & 11 \\ \mathrm{C}(172) & 54 & 54 & 54 & 11 & 4 & 11 \\ \mathrm{C}(173) & 45 & 45 & 45 & 9 & 3 & 9 \\ \mathrm{C}(174) & 44 & 44 & 44 & 9 & 3 & 9 \\ \mathrm{C}(175) & 54 & 54 & 54 & 11 & 4 & 11 \\ \mathrm{C}(176) & 60 & 60 & 60 & 12 & 4 & 12 \\ \mathrm{C}(177) & 70 & 70 & 70 & 14 & 5 & 14 \\ \mathrm{C}(178) & 53 & 53 & 53 & 11 & 4 & 11 \\ \mathrm{C}(179) & 90 & 90 & 90 & 18 & 7 & 18 \\ \mathrm{C}(228) & 102 & 102 & 102 & 21 & 8 & 21 \\ \mathrm{C}(229) & 117 & 117 & 117 & 24 & 9 & 24 \\ \mathrm{C}(230) & 127 & 127 & 127 & 26 & 9 & 26 \\ \mathrm{C}(231) & 109 & 109 & 109 & 22 & 8 & 22 \\ \mathrm{C}(232) & 129 & 129 & 129 & 26 & 10 & 26 \\ \mathrm{C}(233) & 102 & 102 & 102 & 21 & 8 & 21 \\ \mathrm{C}(234) & 138 & 138 & 138 & 28 & 10 & 28 \\ \mathrm{C}(235) & 155 & 155 & 155 & 31 & 12 & 31 \\ \mathrm{C}(236) & 127 & 127 & 127 & 26 & 9 & 26 \\ \mathrm{C}(237) & 102 & 102 & 102 & 21 & 8 & 21 \\ \mathrm{C}(238) & 106 & 106 & 106 & 22 & 8 & 22 \\ \mathrm{C}(239) & 102 & 102 & 102 & 21 & 8 & 21 \\ \mathrm{C}(240) & 52 & 52 & 52 & 11 & 4 & 11 \\ \mathrm{C}(241) & 57 & 57 & 57 & 11 & 4 & 11 \\ \mathrm{C}(247) & 50 & 50 & 50 & 10 & 4 & 10 \\ \mathrm{C}(248) & 47 & 47 & 47 & 10 & 4 & 10 \\ \mathrm{C}(249) & 81 & 81 & 81 & 16 & 6 & 16 \\ \mathrm{C}(250) & 80 & 80 & 80 & 16 & 6 & 16 \\ \mathrm{C}(251) & 66 & 66 & 66 & 13 & 5 & 13 \\ & & & & & & \\ & & & & & & \\ & & 541 & & \end{array}$




$\begin{array}{lcccccc}\mathrm{C}(252) & 75 & 75 & 75 & 15 & 6 & 15 \\ \mathrm{C}(253) & 110 & 110 & 110 & 22 & 8 & 22 \\ \mathrm{C}(254) & 137 & 137 & 137 & 28 & 10 & 28 \\ \mathrm{C}(255) & 89 & 89 & 89 & 18 & 7 & 18 \\ \mathrm{C}(259) & 86 & 86 & 86 & 17 & 6 & 17 \\ \mathrm{~K}(1) & 43 & 43 & 43 & 9 & 3 & 9 \\ \mathrm{~K}(2) & 37 & 37 & 37 & 7 & 3 & 7 \\ \mathrm{~K}(3) & 35 & 35 & 35 & 7 & 3 & 7 \\ \mathrm{~K}(4) & 34 & 34 & 34 & 7 & 3 & 7 \\ \mathrm{~K}(5) & 37 & 37 & 37 & 7 & 3 & 7 \\ \mathrm{~N}(1) & 45 & 45 & 45 & 9 & 3 & 9 \\ \mathrm{~N}(2) & 45 & 45 & 45 & 9 & 3 & 9 \\ \mathrm{~N}(3) & 46 & 46 & 46 & 9 & 3 & 9 \\ \mathrm{~N}(4) & 43 & 43 & 43 & 9 & 3 & 9 \\ \mathrm{~N}(5) & 42 & 42 & 42 & 8 & 3 & 8 \\ \mathrm{~N}(6) & 35 & 35 & 35 & 7 & 3 & 7 \\ \mathrm{~N}(7) & 40 & 40 & 40 & 8 & 3 & 8 \\ \mathrm{~N}(8) & 42 & 42 & 42 & 8 & 3 & 8 \\ \mathrm{~N}(9) & 35 & 35 & 35 & 7 & 3 & 7 \\ \mathrm{~N}(10) & 72 & 72 & 72 & 15 & 5 & 15 \\ \mathrm{~N}(11) & 62 & 62 & 62 & 12 & 5 & 12 \\ \mathrm{~N}(12) & 45 & 45 & 45 & 9 & 3 & 9 \\ \mathrm{O}(1) & 48 & 48 & 48 & 10 & 4 & 10 \\ \mathrm{O}(2) & 52 & 52 & 52 & 11 & 4 & 11 \\ \mathrm{O}(3) & 52 & 52 & 52 & 11 & 4 & 11 \\ \mathrm{O}(16) & 56 & 56 & 56 & 11 & 4 & 11 \\ \mathrm{O}(17) & 52 & 52 & 52 & 11 & 4 & 11 \\ \mathrm{O}(18) & 57 & 57 & 57 & 12 & 4 & 12 \\ \mathrm{O}(19) & 55 & 55 & 55 & 11 & 4 & 11 \\ \mathrm{O}(20) & 51 & 51 & 51 & 10 & 4 & 10 \\ \mathrm{O}(21) & 56 & 56 & 56 & 11 & 4 & 11 \\ \mathrm{O}(22) & 50 & 50 & 50 & 10 & 4 & 10 \\ \mathrm{O}(23) & 53 & 53 & 53 & 11 & 4 & 11 \\ \mathrm{O}(24) & 54 & 54 & 54 & 11 & 4 & 11 \\ \mathrm{O}(25) & 42 & 42 & 42 & 9 & 3 & 9 \\ \mathrm{O}(26) & 54 & 54 & 54 & 11 & 4 & 11 \\ \mathrm{O}(27) & 66 & 66 & 66 & 13 & 5 & 13 \\ \mathrm{O}(28) & 36 & 36 & 36 & 7 & 3 & 7 \\ \mathrm{O}(29) & 44 & 44 & 44 & 9 & 3 & 9 \\ \mathrm{O}(30) & 50 & 50 & 50 & 10 & 4 & 10 \\ \mathrm{O}(31) & 51 & 51 & 51 & 10 & 4 & 10 \\ \mathrm{O}(32) & 51 & 51 & 51 & 10 & 4 & 10 \\ \mathrm{O}(33) & 38 & 38 & 38 & 8 & 3 & 8 \\ \mathrm{O}(34) & 47 & 47 & 47 & 9 & 3 & 9 \\ \mathrm{O}(35) & 48 & 48 & 48 & 10 & 4 & 10 \\ & & & & & & \\ & & & & 542 & & \end{array}$




\begin{tabular}{|c|c|c|c|c|c|c|}
\hline $\mathrm{O}(36)$ & 41 & 41 & 41 & 8 & 3 & 8 \\
\hline $\mathrm{O}(101)$ & 83 & 83 & 83 & 17 & 6 & 17 \\
\hline $\mathrm{O}(102)$ & 98 & 98 & 98 & 20 & 7 & 20 \\
\hline $\mathrm{O}(103)$ & 97 & 97 & 97 & 20 & 7 & 20 \\
\hline $\mathrm{O}(104)$ & 124 & 124 & 124 & 25 & 9 & 25 \\
\hline $\mathrm{O}(105)$ & 102 & 102 & 102 & 21 & 8 & 21 \\
\hline$O(106)$ & 82 & 82 & 82 & 17 & 6 & 17 \\
\hline $\mathrm{O}(107)$ & 49 & 49 & 49 & 10 & 4 & 10 \\
\hline $\mathrm{O}(108)$ & 46 & 46 & 46 & 9 & 3 & 9 \\
\hline $\mathrm{O}(112)$ & 60 & 60 & 60 & 12 & 4 & 12 \\
\hline $\mathrm{O}(113)$ & 95 & 95 & 95 & 19 & 7 & 19 \\
\hline $\mathrm{O}(114)$ & 91 & 91 & 91 & 18 & 7 & 18 \\
\hline $\mathrm{O}(117)$ & 81 & 81 & 81 & 16 & 6 & 16 \\
\hline $\operatorname{Si}(1)$ & 25 & 25 & 25 & 5 & 2 & 5 \\
\hline $\operatorname{Si}(2)$ & 26 & 26 & 26 & 5 & 2 & 5 \\
\hline $\operatorname{Si}(3)$ & 32 & 32 & 32 & 7 & 2 & 7 \\
\hline $\operatorname{Si}(4)$ & 35 & 35 & 35 & 7 & 3 & 7 \\
\hline $\operatorname{Si}(5)$ & 40 & 40 & 40 & 8 & 3 & 8 \\
\hline $\operatorname{Si}(6)$ & 36 & 36 & 36 & 7 & 3 & 7 \\
\hline $\operatorname{Si}(7)$ & 27 & 27 & 27 & 6 & 2 & 6 \\
\hline $\operatorname{Si}(8)$ & 27 & 27 & 27 & 5 & 2 & 5 \\
\hline $\operatorname{Si}(9)$ & 43 & 43 & 43 & 9 & 3 & 9 \\
\hline $\operatorname{Si}(10)$ & 35 & 35 & 35 & 7 & 3 & 7 \\
\hline $\operatorname{Si}(11)$ & 36 & 36 & 36 & 7 & 3 & 7 \\
\hline $\operatorname{Si}(12)$ & 29 & 29 & 29 & 6 & 2 & 6 \\
\hline $\operatorname{Si}(13)$ & 23 & 23 & 23 & 5 & 2 & 5 \\
\hline $\operatorname{Si}(14)$ & 30 & 30 & 30 & 6 & 2 & 6 \\
\hline $\operatorname{Si}(15)$ & 30 & 30 & 30 & 6 & 2 & 6 \\
\hline $\operatorname{Si}(16)$ & 24 & 24 & 24 & 5 & 2 & 5 \\
\hline $\operatorname{Si}(17)$ & 35 & 35 & 35 & 7 & 3 & 7 \\
\hline $\operatorname{Si}(18)$ & 31 & 31 & 31 & 6 & 2 & 6 \\
\hline $\operatorname{Si}(19)$ & 31 & 31 & 31 & 6 & 2 & 6 \\
\hline $\operatorname{Si}(20)$ & 42 & 42 & 42 & 8 & 3 & 8 \\
\hline $\operatorname{Si}(21)$ & 40 & 40 & 40 & 8 & 3 & 8 \\
\hline $\operatorname{Si}(22)$ & 34 & 34 & 34 & 7 & 3 & 7 \\
\hline $\operatorname{Si}(23)$ & 47 & 47 & 47 & 9 & 3 & 9 \\
\hline $\operatorname{Si}(24)$ & 38 & 38 & 38 & 8 & 3 & 8 \\
\hline $\operatorname{Zr}(1)$ & 31 & 36 & 18 & 8 & 2 & 9 \\
\hline $\operatorname{Zr}(2)$ & 29 & 26 & 22 & 5 & 5 & 5 \\
\hline $\operatorname{Zr}(3)$ & 23 & 36 & 20 & 5 & 0 & 4 \\
\hline $\operatorname{Zr}(4)$ & 27 & 37 & 22 & 4 & 1 & 4 \\
\hline
\end{tabular}


Table S15. Hydrogen coordinates $\left(\times 10^{4}\right)$ and isotropic displacement parameters $\left(\AA^{2} \times 10^{3}\right)$ for 3.

\begin{tabular}{|c|c|c|c|c|}
\hline & $\mathrm{x}$ & $\mathrm{y}$ & $\mathrm{z}$ & $\mathrm{U}(\mathrm{eq})$ \\
\hline $\mathrm{H}(1 \mathrm{~A})$ & 5709 & 969 & 8090 & 93 \\
\hline $\mathrm{H}(1 \mathrm{~B})$ & 6128 & 1317 & 8439 & 93 \\
\hline $\mathrm{H}(1 \mathrm{C})$ & 6628 & 1491 & 8130 & 93 \\
\hline $\mathrm{H}(2 \mathrm{~A})$ & 5733 & 1771 & 7747 & 123 \\
\hline $\mathrm{H}(2 \mathrm{~B})$ & 4761 & 2042 & 7832 & 123 \\
\hline $\mathrm{H}(2 \mathrm{C})$ & 4621 & 1388 & 7730 & 123 \\
\hline $\mathrm{H}(3 \mathrm{~A})$ & 1896 & 1033 & 8206 & 113 \\
\hline $\mathrm{H}(3 \mathrm{~B})$ & 2991 & 1072 & 8384 & 113 \\
\hline $\mathrm{H}(3 \mathrm{C})$ & 2860 & 958 & 8001 & 113 \\
\hline $\mathrm{H}(4 \mathrm{~A})$ & 2202 & 1718 & 7768 & 117 \\
\hline $\mathrm{H}(4 \mathrm{~B})$ & 2417 & 2321 & 7989 & 117 \\
\hline $\mathrm{H}(4 \mathrm{C})$ & 1489 & 1861 & 8053 & 117 \\
\hline $\mathrm{H}(5 \mathrm{~A})$ & 5605 & 3365 & 8082 & 122 \\
\hline $\mathrm{H}(5 \mathrm{~B})$ & 6052 & 2830 & 8107 & 122 \\
\hline $\mathrm{H}(5 \mathrm{C})$ & 6277 & 3351 & 8397 & 122 \\
\hline $\mathrm{H}(6 \mathrm{~A})$ & 4368 & 3594 & 8596 & 87 \\
\hline $\mathrm{H}(6 \mathrm{~B})$ & 3443 & 3122 & 8415 & 87 \\
\hline $\mathrm{H}(6 \mathrm{C})$ & 4180 & 3485 & 8215 & 87 \\
\hline $\mathrm{H}(7 \mathrm{~A})$ & 2901 & 599 & 8824 & 71 \\
\hline H(7B) & 3011 & 791 & 9205 & 71 \\
\hline $\mathrm{H}(7 \mathrm{C})$ & 3059 & 172 & 9044 & 71 \\
\hline $\mathrm{H}(8 \mathrm{~A})$ & 4730 & -107 & 8654 & 77 \\
\hline $\mathrm{H}(8 \mathrm{~B})$ & 5811 & 299 & 8660 & 77 \\
\hline $\mathrm{H}(8 \mathrm{C})$ & 4843 & 366 & 8450 & 77 \\
\hline $\mathrm{H}(9 \mathrm{~A})$ & 4999 & 769 & 9580 & 75 \\
\hline $\mathrm{H}(9 \mathrm{~B})$ & 6015 & 688 & 9405 & 75 \\
\hline $\mathrm{H}(9 \mathrm{C})$ & 5071 & 180 & 9375 & 75 \\
\hline $\mathrm{H}(10 \mathrm{~A})$ & 8455 & 1452 & 9028 & 84 \\
\hline $\mathrm{H}(10 \mathrm{~B})$ & 7653 & 1230 & 8721 & 84 \\
\hline $\mathrm{H}(10 \mathrm{C})$ & 7422 & 1022 & 9047 & 84 \\
\hline $\mathrm{H}(11 \mathrm{~A})$ & 7368 & 2862 & 8969 & 115 \\
\hline $\mathrm{H}(11 \mathrm{~B})$ & 7483 & 2420 & 8657 & 115 \\
\hline $\mathrm{H}(11 \mathrm{C})$ & 8385 & 2615 & 8936 & 115 \\
\hline $\mathrm{H}(12 \mathrm{~A})$ & 8154 & 2174 & 9581 & 82 \\
\hline $\mathrm{H}(12 \mathrm{~B})$ & 7000 & 1984 & 9666 & 82 \\
\hline $\mathrm{H}(12 \mathrm{C})$ & 7385 & 2591 & 9604 & 82 \\
\hline $\mathrm{H}(13 \mathrm{~A})$ & 4886 & 1816 & 9585 & 42 \\
\hline $\mathrm{H}(13 \mathrm{~B})$ & 5205 & 2422 & 9514 & 42 \\
\hline $\mathrm{H}(14 \mathrm{~A})$ & 3448 & 2301 & 9627 & 43 \\
\hline $\mathrm{H}(14 \mathrm{~B})$ & 3166 & 1731 & 9370 & 43 \\
\hline
\end{tabular}




\begin{tabular}{|c|c|c|c|c|}
\hline $\mathrm{H}(15 \mathrm{~A})$ & 227 & 1644 & 8992 & 107 \\
\hline $\mathrm{H}(15 \mathrm{~B})$ & 1285 & 1476 & 9078 & 107 \\
\hline $\mathrm{H}(15 \mathrm{C})$ & 1006 & 1540 & 8721 & 107 \\
\hline $\mathrm{H}(16 \mathrm{~A})$ & 1125 & 2608 & 8529 & 100 \\
\hline $\mathrm{H}(16 \mathrm{~B})$ & 1552 & 3189 & 8763 & 100 \\
\hline $\mathrm{H}(16 \mathrm{C})$ & 410 & 2866 & 8784 & 100 \\
\hline $\mathrm{H}(17 \mathrm{~A})$ & 1054 & 3125 & 9485 & 91 \\
\hline $\mathrm{H}(17 \mathrm{~B})$ & 1530 & 2699 & 9646 & 91 \\
\hline $\mathrm{H}(17 \mathrm{C})$ & 389 & 2526 & 9481 & 91 \\
\hline $\mathrm{H}(18 \mathrm{~A})$ & 5812 & 4053 & 9345 & 76 \\
\hline $\mathrm{H}(18 \mathrm{~B})$ & 5577 & 3643 & 9003 & 76 \\
\hline $\mathrm{H}(18 \mathrm{C})$ & 5972 & 3434 & 9307 & 76 \\
\hline $\mathrm{H}(19 \mathrm{~A})$ & 4543 & 3314 & 9872 & 88 \\
\hline $\mathrm{H}(19 B)$ & 3427 & 3454 & 9850 & 88 \\
\hline $\mathrm{H}(19 \mathrm{C})$ & 4421 & 3935 & 9889 & 88 \\
\hline $\mathrm{H}(20 \mathrm{~A})$ & 2713 & 3896 & 9277 & 81 \\
\hline $\mathrm{H}(20 \mathrm{~B})$ & 3421 & 3958 & 8986 & 81 \\
\hline $\mathrm{H}(20 \mathrm{C})$ & 3757 & 4346 & 9331 & 81 \\
\hline $\mathrm{H}(21 \mathrm{~A})$ & 7512 & 89 & 7055 & 85 \\
\hline$H(21 B)$ & 7103 & -171 & 6690 & 85 \\
\hline $\mathrm{H}(21 \mathrm{C})$ & 6457 & -351 & 6975 & 85 \\
\hline$H(22 A)$ & 5680 & 416 & 7337 & 86 \\
\hline $\mathrm{H}(22 \mathrm{~B})$ & 6032 & 1016 & 7261 & 86 \\
\hline $\mathrm{H}(22 \mathrm{C})$ & 6865 & 706 & 7381 & 86 \\
\hline $\mathrm{H}(23 \mathrm{~A})$ & 3216 & 192 & 7051 & 94 \\
\hline $\mathrm{H}(23 \mathrm{~B})$ & 4272 & -16 & 7011 & 94 \\
\hline $\mathrm{H}(23 \mathrm{C})$ & 3356 & -240 & 6737 & 94 \\
\hline $\mathrm{H}(24 \mathrm{~A})$ & 2746 & 712 & 6584 & 83 \\
\hline $\mathrm{H}(24 \mathrm{~B})$ & 3679 & 1226 & 6621 & 83 \\
\hline $\mathrm{H}(24 \mathrm{C})$ & 3178 & 1059 & 6932 & 83 \\
\hline $\mathrm{H}(25 \mathrm{~A})$ & 6980 & 2192 & 6591 & 72 \\
\hline $\mathrm{H}(25 \mathrm{~B})$ & 7224 & 1589 & 6502 & 72 \\
\hline $\mathrm{H}(25 \mathrm{C})$ & 7531 & 1950 & 6855 & 72 \\
\hline $\mathrm{H}(26 \mathrm{~A})$ & 6103 & 2113 & 7221 & 69 \\
\hline $\mathrm{H}(26 \mathrm{~B})$ & 4950 & 1853 & 7080 & 69 \\
\hline $\mathrm{H}(26 \mathrm{C})$ & 5594 & 2358 & 6950 & 69 \\
\hline $\mathrm{H}(27 \mathrm{~A})$ & 4371 & -948 & 6108 & 128 \\
\hline $\mathrm{H}(27 \mathrm{~B})$ & 5056 & -715 & 6439 & 128 \\
\hline $\mathrm{H}(27 \mathrm{C})$ & 4935 & -1346 & 6267 & 128 \\
\hline $\mathrm{H}(28 \mathrm{~A})$ & 5709 & -1487 & 5512 & 114 \\
\hline $\mathrm{H}(28 \mathrm{~B})$ & 6365 & -942 & 5425 & 114 \\
\hline $\mathrm{H}(28 \mathrm{C})$ & 5178 & -980 & 5484 & 114 \\
\hline $\mathrm{H}(29 \mathrm{~A})$ & 7342 & -1081 & 6363 & 107 \\
\hline H(29B) & 7729 & -1178 & 6010 & 107 \\
\hline $\mathrm{H}(29 \mathrm{C})$ & 6788 & -1604 & 6100 & 107 \\
\hline
\end{tabular}




\begin{tabular}{|c|c|c|c|c|}
\hline $\mathrm{H}(30 \mathrm{~A})$ & 9818 & 108 & 5732 & 80 \\
\hline $\mathrm{H}(30 \mathrm{~B})$ & 8729 & 71 & 5551 & 80 \\
\hline $\mathrm{H}(30 \mathrm{C})$ & 8923 & -419 & 5718 & 80 \\
\hline $\mathrm{H}(31 \mathrm{~A})$ & 9940 & 1194 & 6223 & 76 \\
\hline $\mathrm{H}(31 \mathrm{~B})$ & 8889 & 1340 & 6347 & 76 \\
\hline $\mathrm{H}(31 \mathrm{C})$ & 9061 & 1245 & 5973 & 76 \\
\hline $\mathrm{H}(32 \mathrm{~A})$ & 9068 & -253 & 6485 & 101 \\
\hline $\mathrm{H}(32 \mathrm{~B})$ & 9162 & 358 & 6687 & 101 \\
\hline $\mathrm{H}(32 \mathrm{C})$ & 10049 & 211 & 6464 & 101 \\
\hline $\mathrm{H}(33 \mathrm{~A})$ & 7052 & 741 & 5669 & 36 \\
\hline $\mathrm{H}(33 \mathrm{~B})$ & 6855 & 100 & 5509 & 36 \\
\hline $\mathrm{H}(34 \mathrm{~A})$ & 5064 & 7 & 5533 & 46 \\
\hline $\mathrm{H}(34 \mathrm{~B})$ & 5433 & 537 & 5381 & 46 \\
\hline $\mathrm{H}(35 \mathrm{~A})$ & 2453 & -36 & 6137 & 83 \\
\hline $\mathrm{H}(35 \mathrm{~B})$ & 3033 & -378 & 5873 & 83 \\
\hline $\mathrm{H}(35 \mathrm{C})$ & 1899 & -302 & 5789 & 83 \\
\hline $\mathrm{H}(36 \mathrm{~A})$ & 3220 & 50 & 5215 & 85 \\
\hline $\mathrm{H}(36 \mathrm{~B})$ & 3158 & 674 & 5211 & 85 \\
\hline $\mathrm{H}(36 \mathrm{C})$ & 2142 & 239 & 5240 & 85 \\
\hline $\mathrm{H}(37 \mathrm{~A})$ & 1537 & 888 & 5825 & 88 \\
\hline $\mathrm{H}(37 \mathrm{~B})$ & 2472 & 1388 & 5821 & 88 \\
\hline $\mathrm{H}(37 \mathrm{C})$ & 2295 & 1145 & 6138 & 88 \\
\hline $\mathrm{H}(38 \mathrm{~A})$ & 3894 & 1785 & 5438 & 62 \\
\hline $\mathrm{H}(38 \mathrm{~B})$ & 4830 & 1612 & 5247 & 62 \\
\hline $\mathrm{H}(38 \mathrm{C})$ & 4873 & 2243 & 5406 & 62 \\
\hline $\mathrm{H}(39 \mathrm{~A})$ & 6916 & 2379 & 5815 & 63 \\
\hline H(39B) & 6908 & 1807 & 5576 & 63 \\
\hline $\mathrm{H}(39 \mathrm{C})$ & 7115 & 1861 & 5953 & 63 \\
\hline $\mathrm{H}(40 \mathrm{~A})$ & 5327 & 2309 & 6360 & 60 \\
\hline $\mathrm{H}(40 \mathrm{~B})$ & 4182 & 2170 & 6199 & 60 \\
\hline $\mathrm{H}(40 \mathrm{C})$ & 5016 & 2646 & 6104 & 60 \\
\hline $\mathrm{H}(41 \mathrm{~A})$ & 5835 & 3586 & 3026 & 76 \\
\hline $\mathrm{H}(41 \mathrm{~B})$ & 6168 & 3892 & 3389 & 76 \\
\hline $\mathrm{H}(41 \mathrm{C})$ & 6724 & 4126 & 3103 & 76 \\
\hline $\mathrm{H}(42 \mathrm{~A})$ & 5853 & 4524 & 2740 & 105 \\
\hline $\mathrm{H}(42 \mathrm{~B})$ & 4751 & 4664 & 2806 & 105 \\
\hline $\mathrm{H}(42 \mathrm{C})$ & 4852 & 4044 & 2667 & 105 \\
\hline $\mathrm{H}(43 \mathrm{~A})$ & 1915 & 3542 & 3110 & 117 \\
\hline $\mathrm{H}(43 \mathrm{~B})$ & 3025 & 3561 & 3271 & 117 \\
\hline $\mathrm{H}(43 \mathrm{C})$ & 2863 & 3511 & 2893 & 117 \\
\hline $\mathrm{H}(44 \mathrm{~A})$ & 2165 & 4317 & 2725 & 103 \\
\hline $\mathrm{H}(44 \mathrm{~B})$ & 2288 & 4873 & 2988 & 103 \\
\hline $\mathrm{H}(44 \mathrm{C})$ & 1439 & 4348 & 3016 & 103 \\
\hline $\mathrm{H}(45 \mathrm{~A})$ & 5615 & 5773 & 3026 & 100 \\
\hline $\mathrm{H}(45 \mathrm{~B})$ & 6224 & 5543 & 3282 & 100 \\
\hline
\end{tabular}




\begin{tabular}{|c|c|c|c|c|}
\hline $\mathrm{H}(45 \mathrm{C})$ & 5914 & 6130 & 3380 & 100 \\
\hline $\mathrm{H}(46 \mathrm{~A})$ & 4339 & 6229 & 3503 & 70 \\
\hline $\mathrm{H}(46 \mathrm{~B})$ & 3406 & 5723 & 3495 & 70 \\
\hline $\mathrm{H}(46 \mathrm{C})$ & 3759 & 5897 & 3169 & 70 \\
\hline $\mathrm{H}(47 \mathrm{~A})$ & 2966 & 2618 & 3881 & 64 \\
\hline $\mathrm{H}(47 \mathrm{~B})$ & 2803 & 3189 & 3801 & 64 \\
\hline $\mathrm{H}(47 \mathrm{C})$ & 2967 & 3132 & 4165 & 64 \\
\hline $\mathrm{H}(48 \mathrm{~A})$ & 5682 & 2932 & 3504 & 75 \\
\hline $\mathrm{H}(48 \mathrm{~B})$ & 4520 & 2825 & 3361 & 75 \\
\hline $\mathrm{H}(48 \mathrm{C})$ & 4871 & 2416 & 3566 & 75 \\
\hline $\mathrm{H}(49 \mathrm{~A})$ & 5145 & 3274 & 4490 & 72 \\
\hline $\mathrm{H}(49 \mathrm{~B})$ & 5955 & 3034 & 4265 & 72 \\
\hline $\mathrm{H}(49 \mathrm{C})$ & 4882 & 2649 & 4299 & 72 \\
\hline $\mathrm{H}(50 \mathrm{~A})$ & 8376 & 4018 & 4047 & 77 \\
\hline $\mathrm{H}(50 \mathrm{~B})$ & 7675 & 3790 & 3719 & 77 \\
\hline $\mathrm{H}(50 \mathrm{C})$ & 7341 & 3572 & 4035 & 77 \\
\hline $\mathrm{H}(51 \mathrm{~A})$ & 7103 & 5341 & 3869 & 81 \\
\hline $\mathrm{H}(51 \mathrm{~B})$ & 7465 & 4889 & 3605 & 81 \\
\hline $\mathrm{H}(51 \mathrm{C})$ & 8209 & 5199 & 3915 & 81 \\
\hline $\mathrm{H}(52 \mathrm{~A})$ & 8031 & 4845 & 4547 & 75 \\
\hline $\mathrm{H}(52 \mathrm{~B})$ & 6956 & 4526 & 4635 & 75 \\
\hline $\mathrm{H}(52 \mathrm{C})$ & 7061 & 5133 & 4565 & 75 \\
\hline $\mathrm{H}(53 \mathrm{~A})$ & 984 & 3940 & 3958 & 86 \\
\hline $\mathrm{H}(53 \mathrm{~B})$ & 998 & 4057 & 3603 & 86 \\
\hline $\mathrm{H}(53 \mathrm{C})$ & 46 & 4147 & 3810 & 86 \\
\hline $\mathrm{H}(54 \mathrm{~A})$ & 312 & 5383 & 3713 & 85 \\
\hline $\mathrm{H}(54 \mathrm{~B})$ & 1182 & 5239 & 3482 & 85 \\
\hline $\mathrm{H}(54 \mathrm{C})$ & 1431 & 5766 & 3768 & 85 \\
\hline $\mathrm{H}(55 \mathrm{~A})$ & 173 & 4989 & 4380 & 71 \\
\hline $\mathrm{H}(55 \mathrm{~B})$ & 936 & 5574 & 4428 & 71 \\
\hline $\mathrm{H}(55 \mathrm{C})$ & 1276 & 5084 & 4565 & 71 \\
\hline$H(56 A)$ & 5488 & 6041 & 4005 & 68 \\
\hline $\mathrm{H}(56 \mathrm{~B})$ & 5844 & 5897 & 4335 & 68 \\
\hline $\mathrm{H}(56 \mathrm{C})$ & 5659 & 6501 & 4327 & 68 \\
\hline $\mathrm{H}(57 \mathrm{~A})$ & 4400 & 6278 & 4897 & 75 \\
\hline $\mathrm{H}(57 \mathrm{~B})$ & 4312 & 5626 & 4840 & 75 \\
\hline $\mathrm{H}(57 \mathrm{C})$ & 3298 & 5877 & 4846 & 75 \\
\hline $\mathrm{H}(58 \mathrm{~A})$ & 3713 & 6813 & 4356 & 78 \\
\hline $\mathrm{H}(58 \mathrm{~B})$ & 2634 & 6393 & 4313 & 78 \\
\hline $\mathrm{H}(58 \mathrm{C})$ & 3285 & 6453 & 4010 & 78 \\
\hline $\mathrm{H}(59 \mathrm{~A})$ & 3215 & 4696 & 4552 & 33 \\
\hline $\mathrm{H}(59 \mathrm{~B})$ & 2997 & 4154 & 4274 & 33 \\
\hline $\mathrm{H}(60 \mathrm{~A})$ & 4672 & 4220 & 4520 & 35 \\
\hline $\mathrm{H}(60 \mathrm{~B})$ & 5013 & 4845 & 4481 & 35 \\
\hline $\mathrm{H}(61 \mathrm{~A})$ & 5570 & 1453 & 1526 & 197 \\
\hline
\end{tabular}




\begin{tabular}{|c|c|c|c|c|}
\hline $\mathrm{H}(61 \mathrm{~B})$ & 4758 & 1779 & 1697 & 197 \\
\hline $\mathrm{H}(61 \mathrm{C})$ & 5517 & 1540 & 1905 & 197 \\
\hline $\mathrm{H}(62 \mathrm{~A})$ & 7139 & 1857 & 1971 & 179 \\
\hline $\mathrm{H}(62 \mathrm{~B})$ & 7709 & 2490 & 1963 & 179 \\
\hline $\mathrm{H}(63 \mathrm{~A})$ & 4755 & 2828 & 2317 & 177 \\
\hline $\mathrm{H}(63 \mathrm{~B})$ & 4287 & 2287 & 2054 & 177 \\
\hline$H(63 C)$ & 3575 & 2707 & 2190 & 177 \\
\hline $\mathrm{H}(64 \mathrm{~A})$ & 3110 & 3125 & 1827 & 102 \\
\hline $\mathrm{H}(64 \mathrm{~B})$ & 3956 & 3556 & 1699 & 102 \\
\hline $\mathrm{H}(64 \mathrm{C})$ & 3867 & 3600 & 2076 & 102 \\
\hline $\mathrm{H}(65 \mathrm{~A})$ & 7023 & 4154 & 2277 & 120 \\
\hline $\mathrm{H}(65 \mathrm{~B})$ & 5892 & 4054 & 2110 & 120 \\
\hline $\mathrm{H}(65 \mathrm{C})$ & 6805 & 4473 & 2001 & 120 \\
\hline $\mathrm{H}(66 \mathrm{~A})$ & 8169 & 4009 & 1653 & 93 \\
\hline$H(66 B)$ & 8133 & 3381 & 1673 & 93 \\
\hline $\mathrm{H}(66 \mathrm{C})$ & 8352 & 3848 & 1992 & 93 \\
\hline $\mathrm{H}(67 \mathrm{~A})$ & 4145 & 1550 & 707 & 102 \\
\hline $\mathrm{H}(67 \mathrm{~B})$ & 4491 & 1453 & 1051 & 102 \\
\hline $\mathrm{H}(67 \mathrm{C})$ & 4375 & 966 & 743 & 102 \\
\hline $\mathrm{H}(68 \mathrm{~A})$ & 5727 & 1083 & 196 & 96 \\
\hline $\mathrm{H}(68 \mathrm{~B})$ & 6681 & 1585 & 222 & 96 \\
\hline $\mathrm{H}(68 \mathrm{C})$ & 5539 & 1690 & 200 & 96 \\
\hline$H(69 A)$ & 6461 & 723 & 765 & 93 \\
\hline $\mathrm{H}(69 \mathrm{~B})$ & 6714 & 1116 & 1111 & 93 \\
\hline $\mathrm{H}(69 \mathrm{C})$ & 7473 & 1196 & 834 & 93 \\
\hline $\mathrm{H}(70 \mathrm{~A})$ & 9585 & 2343 & 509 & 82 \\
\hline $\mathrm{H}(70 \mathrm{~B})$ & 8471 & 2317 & 348 & 82 \\
\hline $\mathrm{H}(70 \mathrm{C})$ & 8679 & 1831 & 517 & 82 \\
\hline $\mathrm{H}(71 \mathrm{~A})$ & 9080 & 1967 & 1207 & 99 \\
\hline $\mathrm{H}(71 \mathrm{~B})$ & 8899 & 2480 & 1467 & 99 \\
\hline $\mathrm{H}(71 \mathrm{C})$ & 9907 & 2532 & 1273 & 99 \\
\hline $\mathrm{H}(72 \mathrm{~A})$ & 8988 & 3614 & 1161 & 88 \\
\hline $\mathrm{H}(72 \mathrm{~B})$ & 8771 & 3528 & 782 & 88 \\
\hline $\mathrm{H}(72 \mathrm{C})$ & 9827 & 3432 & 927 & 88 \\
\hline $\mathrm{H}(73 \mathrm{~A})$ & 6781 & 3334 & 695 & 51 \\
\hline $\mathrm{H}(73 \mathrm{~B})$ & 6522 & 2766 & 436 & 51 \\
\hline $\mathrm{H}(74 \mathrm{~A})$ & 4822 & 2603 & 559 & 54 \\
\hline $\mathrm{H}(74 \mathrm{~B})$ & 5082 & 3217 & 497 & 54 \\
\hline $\mathrm{H}(75 \mathrm{~A})$ & 1924 & 2901 & 471 & 123 \\
\hline $\mathrm{H}(75 \mathrm{~B})$ & 2659 & 2466 & 419 & 123 \\
\hline $\mathrm{H}(75 \mathrm{C})$ & 3100 & 3096 & 409 & 123 \\
\hline $\mathrm{H}(76 \mathrm{~A})$ & 2661 & 2347 & 1325 & 125 \\
\hline $\mathrm{H}(76 \mathrm{~B})$ & 2682 & 2009 & 969 & 125 \\
\hline $\mathrm{H}(76 \mathrm{C})$ & 1683 & 2237 & 1076 & 125 \\
\hline $\mathrm{H}(77 \mathrm{~A})$ & 2374 & 3848 & 1064 & 111 \\
\hline
\end{tabular}




\begin{tabular}{|c|c|c|c|c|}
\hline $\mathrm{H}(77 \mathrm{~B})$ & 2310 & 3587 & 1378 & 111 \\
\hline $\mathrm{H}(77 \mathrm{C})$ & 1455 & 3350 & 1087 & 111 \\
\hline $\mathrm{H}(78 \mathrm{~A})$ & 4843 & 4878 & 766 & 92 \\
\hline $\mathrm{H}(78 \mathrm{~B})$ & 3859 & 4393 & 736 & 92 \\
\hline $\mathrm{H}(78 \mathrm{C})$ & 4833 & 4292 & 542 & 92 \\
\hline $\mathrm{H}(79 \mathrm{~A})$ & 6919 & 4304 & 934 & 90 \\
\hline $\mathrm{H}(79 \mathrm{~B})$ & 6963 & 4473 & 1316 & 90 \\
\hline $\mathrm{H}(79 \mathrm{C})$ & 6766 & 4902 & 1102 & 90 \\
\hline $\mathrm{H}(80 \mathrm{~A})$ & 5152 & 4669 & 1672 & 80 \\
\hline $\mathrm{H}(80 \mathrm{~B})$ & 4005 & 4502 & 1511 & 80 \\
\hline $\mathrm{H}(80 \mathrm{C})$ & 4749 & 5046 & 1454 & 80 \\
\hline $\mathrm{H}(81 \mathrm{~A})$ & 6577 & 4219 & 9992 & 76 \\
\hline $\mathrm{H}(81 \mathrm{~B})$ & 7374 & 4471 & 10299 & 76 \\
\hline $\mathrm{H}(82 \mathrm{~A})$ & 7450 & 3531 & 10094 & 82 \\
\hline $\mathrm{H}(82 \mathrm{~B})$ & 7759 & 3682 & 9758 & 82 \\
\hline $\mathrm{H}(83 \mathrm{~A})$ & 9431 & 3425 & 9808 & 69 \\
\hline $\mathrm{H}(83 \mathrm{~B})$ & 9016 & 3213 & 10120 & 69 \\
\hline $\mathrm{H}(84 \mathrm{~A})$ & 10431 & 3831 & 10438 & 71 \\
\hline $\mathrm{H}(84 \mathrm{~B})$ & 10813 & 3389 & 10172 & 71 \\
\hline $\mathrm{H}(85 \mathrm{~A})$ & 12448 & 4061 & 10229 & 74 \\
\hline $\mathrm{H}(85 \mathrm{~B})$ & 12034 & 4544 & 10458 & 74 \\
\hline $\mathrm{H}(86 \mathrm{~A})$ & 13385 & 4897 & 10144 & 74 \\
\hline $\mathrm{H}(86 \mathrm{~B})$ & 12649 & 4618 & 9827 & 74 \\
\hline $\mathrm{H}(88)$ & 9449 & 5396 & 8913 & 58 \\
\hline $\mathrm{H}(89)$ & 11063 & 5328 & 9105 & 81 \\
\hline $\mathrm{H}(90)$ & 11236 & 4498 & 9285 & 101 \\
\hline $\mathrm{H}(91)$ & 9760 & 3788 & 9253 & 95 \\
\hline $\mathrm{H}(92)$ & 8181 & 3861 & 9044 & 67 \\
\hline $\mathrm{H}(93 \mathrm{~A})$ & 7585 & 4599 & 8571 & 123 \\
\hline $\mathrm{H}(93 \mathrm{~B})$ & 7496 & 5067 & 8873 & 123 \\
\hline $\mathrm{H}(93 \mathrm{C})$ & 7048 & 4435 & 8878 & 123 \\
\hline $\mathrm{H}(99 \mathrm{~A})$ & 1678 & 3819 & 8319 & 75 \\
\hline $\mathrm{H}(99 \mathrm{~B})$ & 1574 & 4452 & 8368 & 75 \\
\hline $\mathrm{H}(11 \mathrm{D})$ & 1237 & 4274 & 4860 & 80 \\
\hline $\mathrm{H}(11 \mathrm{E})$ & 1177 & 3779 & 4559 & 80 \\
\hline $\mathrm{H}(11 \mathrm{~F})$ & 2696 & 3899 & 4885 & 71 \\
\hline $\mathrm{H}(11 \mathrm{G})$ & 2036 & 3790 & 5181 & 71 \\
\hline $\mathrm{H}(12 \mathrm{D})$ & 2934 & 3031 & 5201 & 69 \\
\hline $\mathrm{H}(12 \mathrm{E})$ & 3493 & 3108 & 4882 & 69 \\
\hline $\mathrm{H}(12 \mathrm{~F})$ & 2631 & 2217 & 4625 & 77 \\
\hline $\mathrm{H}(12 \mathrm{G})$ & 3330 & 2198 & 4935 & 77 \\
\hline $\mathrm{H}(12 \mathrm{H})$ & 2265 & 1328 & 4979 & 92 \\
\hline $\mathrm{H}(12 \mathrm{I})$ & 1523 & 1332 & 4676 & 92 \\
\hline $\mathrm{H}(12 \mathrm{~J})$ & 695 & 813 & 5029 & 89 \\
\hline $\mathrm{H}(12 \mathrm{~K})$ & 886 & 1358 & 5304 & 89 \\
\hline
\end{tabular}




\begin{tabular}{|c|c|c|c|c|}
\hline $\mathrm{H}(12 \mathrm{~L})$ & -1020 & 1310 & 5331 & 76 \\
\hline $\mathrm{H}(12 \mathrm{M})$ & -1120 & 731 & 5089 & 76 \\
\hline $\mathrm{H}(12 \mathrm{~N})$ & -2032 & 1060 & 4711 & 78 \\
\hline $\mathrm{H}(12 \mathrm{O})$ & -2611 & 1045 & 5033 & 78 \\
\hline $\mathrm{H}(12 \mathrm{P})$ & -3453 & 1796 & 4909 & 75 \\
\hline $\mathrm{H}(12 \mathrm{Q})$ & -2817 & 1851 & 4601 & 75 \\
\hline $\mathrm{H}(12 \mathrm{R})$ & -3311 & 2683 & 4820 & 76 \\
\hline $\mathrm{H}(12 \mathrm{~S})$ & -2676 & 2708 & 5150 & 76 \\
\hline $\mathrm{H}(12 \mathrm{~T})$ & -1658 & 3593 & 5095 & 79 \\
\hline $\mathrm{H}(12 \mathrm{U})$ & -2259 & 3559 & 4757 & 79 \\
\hline $\mathrm{H}(12 \mathrm{~V})$ & -723 & 3570 & 4506 & 91 \\
\hline $\mathrm{H}(12 \mathrm{~W})$ & -648 & 4113 & 4776 & 91 \\
\hline $\mathrm{H}(131)$ & -758 & 2899 & 3907 & 60 \\
\hline $\mathrm{H}(132)$ & 888 & 2833 & 4072 & 66 \\
\hline H(133) & 1188 & 2013 & 4205 & 78 \\
\hline $\mathrm{H}(134)$ & -171 & 1270 & 4202 & 76 \\
\hline $\mathrm{H}(135)$ & -1884 & 1334 & 4020 & 70 \\
\hline $\mathrm{H}(13 \mathrm{C})$ & -2826 & 2460 & 4019 & 157 \\
\hline $\mathrm{H}(13 \mathrm{D})$ & -3063 & 1826 & 3845 & 157 \\
\hline $\mathrm{H}(13 \mathrm{E})$ & -2627 & 2289 & 3649 & 157 \\
\hline H(138) & 1997 & 3620 & 5789 & 44 \\
\hline $\mathrm{H}(139)$ & 375 & 3796 & 5630 & 64 \\
\hline $\mathrm{H}(140)$ & -1145 & 3139 & 5617 & 74 \\
\hline $\mathrm{H}(141)$ & -1068 & 2262 & 5723 & 62 \\
\hline $\mathrm{H}(142)$ & 554 & 2078 & 5889 & 52 \\
\hline $\mathrm{H}(14 \mathrm{C})$ & 2342 & 2359 & 6042 & 85 \\
\hline $\mathrm{H}(14 \mathrm{D})$ & 2835 & 3002 & 6118 & 85 \\
\hline $\mathrm{H}(14 \mathrm{E})$ & 2876 & 2642 & 5769 & 85 \\
\hline $\mathrm{H}(14 \mathrm{~F})$ & -1056 & 1603 & 7088 & 69 \\
\hline $\mathrm{H}(14 \mathrm{G})$ & -687 & 1240 & 7323 & 69 \\
\hline $\mathrm{H}(14 \mathrm{H})$ & 1077 & 1662 & 7272 & 73 \\
\hline $\mathrm{H}(14 \mathrm{I})$ & 531 & 1323 & 6932 & 73 \\
\hline $\mathrm{H}(14 \mathrm{~J})$ & 1528 & 2479 & 6638 & 74 \\
\hline $\mathrm{H}(14 \mathrm{~K})$ & 1627 & 1860 & 6644 & 74 \\
\hline $\mathrm{H}(14 \mathrm{~L})$ & 2701 & 2166 & 7122 & 78 \\
\hline $\mathrm{H}(14 \mathrm{M})$ & 3183 & 2469 & 6849 & 78 \\
\hline $\mathrm{H}(14 \mathrm{~N})$ & 4130 & 3210 & 7287 & 60 \\
\hline $\mathrm{H}(140)$ & 3568 & 2932 & 7559 & 60 \\
\hline $\mathrm{H}(14 \mathrm{P})$ & 4051 & 3923 & 7738 & 61 \\
\hline $\mathrm{H}(14 \mathrm{Q})$ & 3339 & 3997 & 7445 & 61 \\
\hline $\mathrm{H}(15 \mathrm{D})$ & 2334 & 4478 & 7837 & 62 \\
\hline $\mathrm{H}(15 \mathrm{E})$ & 3141 & 4398 & 8104 & 62 \\
\hline $\mathrm{H}(15 \mathrm{~F})$ & 18 & 4057 & 8422 & 147 \\
\hline $\mathrm{H}(15 \mathrm{G})$ & -614 & 4212 & 8143 & 147 \\
\hline $\mathrm{H}(15 \mathrm{H})$ & -1601 & 3572 & 8200 & 213 \\
\hline
\end{tabular}




\begin{tabular}{|c|c|c|c|c|}
\hline $\mathrm{H}(15 \mathrm{I})$ & -767 & 3307 & 8356 & 213 \\
\hline $\mathrm{H}(15 \mathrm{~J})$ & -1884 & 2473 & 8082 & 91 \\
\hline $\mathrm{H}(15 \mathrm{~K})$ & -689 & 2452 & 8104 & 91 \\
\hline $\mathrm{H}(15 \mathrm{~L})$ & -1692 & 1683 & 7713 & 74 \\
\hline $\mathrm{H}(15 \mathrm{M})$ & -1936 & 2117 & 7506 & 74 \\
\hline $\mathrm{H}(15 \mathrm{~N})$ & 1922 & 3953 & 6619 & 54 \\
\hline $\mathrm{H}(15 \mathrm{O})$ & 2237 & 3960 & 6985 & 54 \\
\hline $\mathrm{H}(15 \mathrm{P})$ & 1959 & 4859 & 6953 & 47 \\
\hline $\mathrm{H}(15 \mathrm{Q})$ & 835 & 4603 & 6780 & 47 \\
\hline $\mathrm{H}(15 \mathrm{R})$ & 97 & 5122 & 7186 & 59 \\
\hline $\mathrm{H}(15 \mathrm{~S})$ & 1171 & 5389 & 7390 & 59 \\
\hline $\mathrm{H}(15 \mathrm{~T})$ & -568 & 4730 & 7635 & 61 \\
\hline $\mathrm{H}(15 \mathrm{U})$ & 520 & 4966 & 7838 & 61 \\
\hline $\mathrm{H}(15 \mathrm{~V})$ & -497 & 6025 & 8202 & 76 \\
\hline $\mathrm{H}(15 \mathrm{~W})$ & -421 & 5406 & 8219 & 76 \\
\hline $\mathrm{H}(16 \mathrm{D})$ & -2185 & 5574 & 8246 & 69 \\
\hline $\mathrm{H}(16 \mathrm{E})$ & -2150 & 5645 & 7881 & 69 \\
\hline $\mathrm{H}(16 \mathrm{~F})$ & -3307 & 4896 & 7633 & 69 \\
\hline $\mathrm{H}(16 \mathrm{G})$ & -3665 & 4718 & 7960 & 69 \\
\hline $\mathrm{H}(16 \mathrm{H})$ & -3088 & 3852 & 7788 & 65 \\
\hline $\mathrm{H}(16 \mathrm{I})$ & -3999 & 3905 & 7547 & 65 \\
\hline $\mathrm{H}(16 \mathrm{~J})$ & -3141 & 3248 & 7169 & 60 \\
\hline $\mathrm{H}(16 \mathrm{~K})$ & -2168 & 3344 & 7422 & 60 \\
\hline $\mathrm{H}(16 \mathrm{~L})$ & -2115 & 3543 & 6769 & 55 \\
\hline $\mathrm{H}(16 \mathrm{M})$ & -1197 & 3883 & 7027 & 55 \\
\hline $\mathrm{H}(16 \mathrm{~N})$ & -1377 & 2879 & 6450 & 49 \\
\hline $\mathrm{H}(160)$ & -548 & 2605 & 6605 & 49 \\
\hline $\mathrm{H}(16 \mathrm{P})$ & 324 & 3327 & 6369 & 45 \\
\hline $\mathrm{H}(16 \mathrm{Q})$ & -243 & 3759 & 6582 & 45 \\
\hline $\mathrm{H}(16 \mathrm{R})$ & -2409 & 551 & 192 & 59 \\
\hline $\mathrm{H}(16 \mathrm{~S})$ & -3210 & 352 & -117 & 59 \\
\hline $\mathrm{H}(16 \mathrm{~T})$ & -1833 & 629 & -436 & 70 \\
\hline $\mathrm{H}(16 \mathrm{U})$ & -2122 & 1135 & -192 & 70 \\
\hline $\mathrm{H}(16 \mathrm{~V})$ & -411 & 1700 & -160 & 66 \\
\hline $\mathrm{H}(16 \mathrm{~W})$ & -82 & 1229 & -421 & 66 \\
\hline H(17D) & 1361 & 1788 & -88 & 65 \\
\hline $\mathrm{H}(17 \mathrm{E})$ & 858 & 1591 & 217 & 65 \\
\hline $\mathrm{H}(17 \mathrm{~F})$ & 2386 & 1173 & 304 & 66 \\
\hline $\mathrm{H}(17 \mathrm{G})$ & 2847 & 1380 & -4 & 66 \\
\hline $\mathrm{H}(17 \mathrm{H})$ & 2755 & 445 & -261 & 64 \\
\hline $\mathrm{H}(17 \mathrm{I})$ & 3511 & 614 & 59 & 64 \\
\hline $\mathrm{H}(174)$ & 638 & -443 & 1035 & 53 \\
\hline $\mathrm{H}(175)$ & -1028 & -433 & 867 & 65 \\
\hline $\mathrm{H}(176)$ & -1406 & 350 & 689 & 72 \\
\hline $\mathrm{H}(177)$ & -58 & 1149 & 723 & 83 \\
\hline
\end{tabular}




\begin{tabular}{|c|c|c|c|c|}
\hline H(178) & 1616 & 1127 & 905 & 64 \\
\hline $\mathrm{H}(17 \mathrm{~J})$ & 2496 & 478 & 1350 & 135 \\
\hline $\mathrm{H}(17 \mathrm{~K})$ & 2895 & 599 & 1016 & 135 \\
\hline $\mathrm{H}(17 \mathrm{~L})$ & 2558 & -21 & 1063 & 135 \\
\hline $\mathrm{H}(228)$ & 8859 & 671 & 1457 & 122 \\
\hline H(229) & 10735 & 1023 & 1746 & 141 \\
\hline $\mathrm{H}(230)$ & 11710 & 1933 & 2056 & 152 \\
\hline $\mathrm{H}(23 \mathrm{D})$ & 10522 & 2639 & 1910 & 131 \\
\hline $\mathrm{H}(23 \mathrm{E})$ & 11566 & 2843 & 2132 & 131 \\
\hline $\mathrm{H}(232)$ & 10185 & 3505 & 2349 & 154 \\
\hline $\mathrm{H}(233)$ & 9755 & 3566 & 2902 & 123 \\
\hline $\mathrm{H}(23 \mathrm{~F})$ & 7893 & 3126 & 3179 & 165 \\
\hline $\mathrm{H}(23 \mathrm{G})$ & 8359 & 2589 & 3196 & 165 \\
\hline $\mathrm{H}(235)$ & 6333 & 2534 & 2950 & 186 \\
\hline $\mathrm{H}(236)$ & 5429 & 1593 & 2751 & 152 \\
\hline $\mathrm{H}(237)$ & 5994 & 669 & 2725 & 123 \\
\hline $\mathrm{H}(238)$ & 6756 & 104 & 2247 & 128 \\
\hline $\mathrm{H}(239)$ & 7143 & 298 & 1668 & 122 \\
\hline $\mathrm{H}(24 \mathrm{D})$ & 9934 & 1666 & 3467 & 63 \\
\hline $\mathrm{H}(24 \mathrm{E})$ & 10575 & 1282 & 3247 & 63 \\
\hline $\mathrm{H}(24 \mathrm{~F})$ & 8890 & 780 & 3277 & 68 \\
\hline $\mathrm{H}(24 \mathrm{G})$ & 8415 & 1234 & 3144 & 68 \\
\hline $\mathrm{H}(24 \mathrm{H})$ & 10988 & 2503 & 3322 & 60 \\
\hline $\mathrm{H}(24 \mathrm{I})$ & 11043 & 2526 & 2952 & 60 \\
\hline $\mathrm{H}(24 \mathrm{~J})$ & 12633 & 2391 & 3153 & 56 \\
\hline $\mathrm{H}(24 \mathrm{~K})$ & 12069 & 1844 & 3264 & 56 \\
\hline $\mathrm{H}(24 \mathrm{~L})$ & 9767 & 197 & 2940 & 97 \\
\hline $\mathrm{H}(24 \mathrm{M})$ & 10456 & 667 & 2791 & 97 \\
\hline $\mathrm{H}(25 \mathrm{D})$ & 8950 & -242 & 2420 & 96 \\
\hline $\mathrm{H}(25 \mathrm{E})$ & 9571 & 243 & 2266 & 96 \\
\hline $\mathrm{H}(25 \mathrm{~F})$ & 13166 & 1345 & 2901 & 80 \\
\hline $\mathrm{H}(25 \mathrm{G})$ & 13618 & 1849 & 2732 & 80 \\
\hline $\mathrm{H}(25 \mathrm{H})$ & 12220 & 854 & 2402 & 89 \\
\hline $\mathrm{H}(25 \mathrm{I})$ & 12668 & 1358 & 2234 & 89 \\
\hline $\mathrm{H}(253)$ & 9791 & -733 & 1935 & 132 \\
\hline $\mathrm{H}(25 \mathrm{~J})$ & 11441 & -767 & 1772 & 164 \\
\hline $\mathrm{H}(25 \mathrm{~K})$ & 11812 & -674 & 2143 & 164 \\
\hline $\mathrm{H}(255)$ & 13231 & -303 & 2021 & 107 \\
\hline $\mathrm{H}(25 \mathrm{~L})$ & 13360 & 736 & 1857 & 103 \\
\hline $\mathrm{H}(25 \mathrm{M})$ & 14330 & 516 & 1971 & 103 \\
\hline
\end{tabular}


Table S16. Crystal data and structure refinement for 4.

\begin{tabular}{|c|c|c|}
\hline Empirical formula & \multicolumn{2}{|c|}{ C180 H420 Hf4 K4 N12 O36 Si24 } \\
\hline Formula weight & \multicolumn{2}{|l|}{4873.80} \\
\hline Temperature & \multicolumn{2}{|l|}{$173(2) \mathrm{K}$} \\
\hline Wavelength & \multicolumn{2}{|l|}{$0.71073 \AA$} \\
\hline Crystal system & \multicolumn{2}{|l|}{ Triclinic } \\
\hline Space group & \multicolumn{2}{|l|}{ P-1 } \\
\hline \multirow[t]{3}{*}{ Unit cell dimensions } & $a=13.114(7) \AA$ & $\alpha=100.870(9)^{\circ}$ \\
\hline & $\mathrm{b}=24.704(14) \AA$ & $\beta=91.881(9)^{\circ}$ \\
\hline & $\mathrm{c}=41.71(2) \AA$ & $\gamma=101.425(10)^{\circ}$ \\
\hline Volume & \multicolumn{2}{|l|}{$12974(12) \AA^{3}$} \\
\hline Z & \multicolumn{2}{|l|}{2} \\
\hline Density (calculated) & \multicolumn{2}{|l|}{$1.248 \mathrm{~g} / \mathrm{cm}^{3}$} \\
\hline Absorption coefficient & \multicolumn{2}{|l|}{$1.828 \mathrm{~mm}^{-1}$} \\
\hline $\mathrm{F}(000)$ & \multicolumn{2}{|l|}{5144} \\
\hline Crystal size & \multicolumn{2}{|c|}{$0.30 \times 0.25 \times 0.23 \mathrm{~mm}^{3}$} \\
\hline Theta range for data collection & \multicolumn{2}{|l|}{1.00 to $28.99^{\circ}$} \\
\hline Index ranges & \multicolumn{2}{|c|}{$-17 \leq \mathrm{h} \leq 17,-33 \leq \mathrm{k} \leq 33,-55 \leq 1 \leq 55$} \\
\hline Reflections collected & \multicolumn{2}{|l|}{141993} \\
\hline Independent reflections & \multicolumn{2}{|c|}{$61492[\mathrm{R}(\mathrm{int})=0.0670]$} \\
\hline Completeness to theta $=28.99^{\circ}$ & \multicolumn{2}{|l|}{$89.2 \%$} \\
\hline Max. and min. transmission & \multicolumn{2}{|l|}{0.6785 and 0.6101} \\
\hline Refinement method & \multicolumn{2}{|c|}{ Full-matrix-block least-squares on $\mathrm{F}^{2}$} \\
\hline Data / restraints / parameters & \multicolumn{2}{|l|}{$61492 / 0 / 2420$} \\
\hline Goodness-of-fit on $\mathrm{F}^{2}$ & \multicolumn{2}{|l|}{0.965} \\
\hline Final $R$ indices $[\mathrm{I}>2 \operatorname{sigma}(\mathrm{I})]$ & \multicolumn{2}{|c|}{$\mathrm{R} 1=0.0621, \mathrm{wR} 2=0.1476$} \\
\hline $\mathrm{R}$ indices (all data) & \multicolumn{2}{|c|}{$\mathrm{R} 1=0.1256, \mathrm{wR} 2=0.2000$} \\
\hline Largest diff. peak and hole & \multicolumn{2}{|c|}{3.759 and -1.707 e. $\AA^{-3}$} \\
\hline
\end{tabular}


Table S17. Atomic coordinates $\left(\times 10^{4}\right)$ and equivalent isotropic displacement parameters $\left(\AA^{2} \times\right.$ $10^{3}$ ) for 4 . $\mathrm{U}(\mathrm{eq})$ is defined as one third of the trace of the orthogonalized Uij tensor.

\begin{tabular}{|c|c|c|c|c|}
\hline & $\mathrm{X}$ & $\mathrm{y}$ & $\mathrm{z}$ & $\mathrm{U}(\mathrm{eq})$ \\
\hline $\mathrm{C}(1)$ & $-56(10)$ & $8293(6)$ & $7152(2)$ & 83 \\
\hline$C(2)$ & $-1037(8)$ & $8624(5)$ & $6782(3)$ & 60 \\
\hline$C(3)$ & 2361(9) & $8867(4)$ & $6803(3)$ & 76 \\
\hline$C(4)$ & $2783(9)$ & $8049(5)$ & $6988(3)$ & 76 \\
\hline$C(5)$ & $-773(8)$ & $6882(5)$ & $6762(3)$ & 78 \\
\hline$C(6)$ & $861(8)$ & $6678(4)$ & $6584(2)$ & 55 \\
\hline$C(7)$ & $1376(6)$ & $7888(4)$ & $5582(2)$ & 34 \\
\hline $\mathrm{C}(8)$ & $221(6)$ & $7936(4)$ & $5561(2)$ & 37 \\
\hline C(9) & $4048(7)$ & $8296(4)$ & $6066(3)$ & 74 \\
\hline$C(10)$ & $3897(7)$ & $7152(5)$ & $6245(3)$ & 62 \\
\hline $\mathrm{C}(11)$ & 3914(8) & $7271(5)$ & $5534(3)$ & 64 \\
\hline$C(12)$ & $1572(8)$ & $6004(4)$ & $5778(3)$ & 58 \\
\hline$C(13)$ & $-584(7)$ & $6320(4)$ & $5752(2)$ & 52 \\
\hline$C(14)$ & $855(8)$ & $6446(5)$ & $5202(2)$ & 60 \\
\hline$C(15)$ & $1743(6)$ & $9432(4)$ & $5976(2)$ & 47 \\
\hline$C(16)$ & $-70(7)$ & $9711(4)$ & $6345(2)$ & 46 \\
\hline $\mathrm{C}(17)$ & $-261(7)$ & $9417(4)$ & $5607(2)$ & 46 \\
\hline $\mathrm{C}(18)$ & $-2742(7)$ & $8641(4)$ & $6031(3)$ & 56 \\
\hline$C(19)$ & $-2636(8)$ & $7451(5)$ & $6103(3)$ & 78 \\
\hline $\mathrm{C}(20)$ & $-2439(7)$ & 7788(4) & $5458(2)$ & 53 \\
\hline$C(21)$ & $8158(9)$ & $10054(4)$ & $8092(3)$ & 65 \\
\hline$C(22)$ & $8773(9)$ & $9327(5)$ & $7750(2)$ & 62 \\
\hline$C(23)$ & $7979(6)$ & $8150(4)$ & $8335(2)$ & 46 \\
\hline $\mathrm{C}(24)$ & $9356(9)$ & $7996(4)$ & $7975(2)$ & 56 \\
\hline$C(25)$ & $11248(9)$ & $9900(4)$ & $8102(3)$ & 72 \\
\hline$C(26)$ & $11618(7)$ & $9086(4)$ & $8287(3)$ & 53 \\
\hline$C(27)$ & $9539(6)$ & $9613(4)$ & $9419(2)$ & 36 \\
\hline $\mathrm{C}(28)$ & $8400(6)$ & $9609(4)$ & $9326(2)$ & 34 \\
\hline C(29) & $8287(6)$ & $8029(3)$ & $9217(2)$ & 39 \\
\hline$C(30)$ & $10108(7)$ & $7720(3)$ & $8833(2)$ & 37 \\
\hline $\mathrm{C}(31)$ & $10381(7)$ & $8141(4)$ & $9571(2)$ & 47 \\
\hline$C(32)$ & $12757(7)$ & $8948(4)$ & $9101(3)$ & 56 \\
\hline C(33) & $12137(8)$ & $9651(4)$ & $9702(2)$ & 54 \\
\hline C(34) & $12429(7)$ & $10131(4)$ & $9091(2)$ & 52 \\
\hline$C(35)$ & $5807(6)$ & $8884(3)$ & $8830(2)$ & 46 \\
\hline$C(36)$ & $5709(8)$ & 9881(4) & $8518(3)$ & 65 \\
\hline$C(37)$ & $5906(7)$ & $10027(4)$ & $9264(2)$ & 52 \\
\hline $\mathrm{C}(38)$ & 7861(9) & $11192(4)$ & $8865(3)$ & 70 \\
\hline$C(39)$ & $10057(9)$ & $10963(5)$ & $8781(4)$ & 96 \\
\hline $\mathrm{C}(40)$ & $9147(9)$ & $11079(4)$ & $9449(3)$ & 76 \\
\hline
\end{tabular}




\begin{tabular}{|c|c|c|c|}
\hline$C(41)$ & $7662(9)$ & $3672(4)$ & $8088(3)$ \\
\hline$C(42)$ & $7123(8)$ & $4492(5)$ & $7974(3)$ \\
\hline$C(43)$ & $8971(7)$ & $5862(4)$ & $8382(2)$ \\
\hline$C(44)$ & $10668(7)$ & $5732(4)$ & $8243(3)$ \\
\hline$C(45)$ & $10173(8)$ & $4385(5)$ & $7807(2)$ \\
\hline$C(46)$ & $11055(7)$ & $3962(4)$ & $8164(2)$ \\
\hline$C(47)$ & $9589(6)$ & $4492(3)$ & $9378(2)$ \\
\hline$C(48)$ & $8431(5)$ & $4522(3)$ & $9333(2)$ \\
\hline$C(49)$ & $8178(6)$ & $3025(3)$ & $8938(2)$ \\
\hline$C(50)$ & $9958(7)$ & $2789(4)$ & $8554(2)$ \\
\hline$C(51)$ & $10215(7)$ & $3010(4)$ & $9288(2)$ \\
\hline$C(52)$ & $12660(6)$ & $3897(4)$ & $8948(3)$ \\
\hline$C(53)$ & $12487(7)$ & $5053(4)$ & $8833(2)$ \\
\hline$C(54)$ & $12313(7)$ & 4777(4) & $9510(2)$ \\
\hline$C(55)$ & 5791(7) & $4182(4)$ & $8821(3)$ \\
\hline$C(56)$ & $6040(7)$ & $5400(5)$ & $8708(3)$ \\
\hline$C(57)$ & $5904(7)$ & $5182(4)$ & $9392(2)$ \\
\hline$C(58)$ & $10450(6)$ & $6109(4)$ & $9240(2)$ \\
\hline$C(59)$ & 8995(8) & $5919(4)$ & $9786(2)$ \\
\hline$C(60)$ & $8298(8)$ & $6456(4)$ & $9242(2)$ \\
\hline$C(61)$ & $2986(7)$ & $3700(5)$ & $6771(3)$ \\
\hline$C(62)$ & $1642(9)$ & $4084(5)$ & $7090(3)$ \\
\hline$C(63)$ & $-663(12)$ & $2695(7)$ & $7151(4)$ \\
\hline$C(64)$ & $-1207(8)$ & $3339(5)$ & $6863(3)$ \\
\hline$C(65)$ & $2113(11)$ & $2174(8)$ & $6907(5)$ \\
\hline$C(66)$ & $398(14)$ & $1681(5)$ & $6717(4)$ \\
\hline$C(67)$ & $216(7)$ & $2966(4)$ & $5648(2)$ \\
\hline$C(68)$ & $1360(7)$ & $2956(4)$ & $5652(2)$ \\
\hline$C(69)$ & $4104(8)$ & $3388(4)$ & $5955(3)$ \\
\hline$C(70)$ & $4173(7)$ & $2371(5)$ & $6266(2)$ \\
\hline$C(71)$ & $3840(7)$ & $2226(4)$ & $5527(2)$ \\
\hline$C(72)$ & 1761(8) & $1111(4)$ & $5897(3)$ \\
\hline$C(73)$ & $-447(7)$ & $1375(5)$ & $5820(3)$ \\
\hline$C(74)$ & $987(8)$ & $1472(5)$ & $5289(2)$ \\
\hline$C(75)$ & $1619(7)$ & $4508(4)$ & $6123(3)$ \\
\hline$C(76)$ & $-409(8)$ & $4458(4)$ & $5744(2)$ \\
\hline$C(77)$ & $-263(8)$ & $4650(4)$ & $6492(2)$ \\
\hline $\mathrm{C}(78)$ & $-2823(7)$ & $3498(4)$ & $6155(4)$ \\
\hline$C(79)$ & $-2584(9)$ & $2307(4)$ & $6103(4)$ \\
\hline $\mathrm{C}(80)$ & $-2397(9)$ & $2840(5)$ & $5515(3)$ \\
\hline $\mathrm{C}(81)$ & $1563(7)$ & $6260(4)$ & $7401(2)$ \\
\hline$C(82)$ & $1495(7)$ & $6816(4)$ & $7585(2)$ \\
\hline$C(83)$ & $2401(8)$ & $7575(5)$ & $7996(3)$ \\
\hline$C(84)$ & $3366(8)$ & $7774(5)$ & $8210(2)$ \\
\hline$C(85)$ & $4464(8)$ & $8345(4)$ & $7880(3)$ \\
\hline
\end{tabular}




\begin{tabular}{|c|c|c|c|}
\hline$C(86)$ & $5492(7)$ & $8393(4)$ & $7739(2)$ \\
\hline$C(87)$ & $6386(7)$ & $7949(4)$ & $7339(3)$ \\
\hline $\mathrm{C}(88)$ & $6259(9)$ & $7496(5)$ & $7036(3)$ \\
\hline C(89) & $5934(12)$ & $6485(7)$ & $6864(4)$ \\
\hline$C(90)$ & $5200(11)$ & $6094(6)$ & $6802(4)$ \\
\hline $\mathrm{C}(91)$ & $3404(8)$ & $5857(5)$ & $6802(2)$ \\
\hline$C(92)$ & $2528(7)$ & $5761(4)$ & $7024(3)$ \\
\hline C(93) & $5805(7)$ & $7053(4)$ & $8370(2)$ \\
\hline C(94) & $4950(6)$ & $6576(4)$ & $8421(2)$ \\
\hline C(95) & $3336(6)$ & $6016(3)$ & $8160(2)$ \\
\hline C(96) & $3661(7)$ & $5450(4)$ & $8057(2)$ \\
\hline$C(97)$ & $4412(7)$ & $4920(4)$ & $7652(2)$ \\
\hline $\mathrm{C}(98)$ & $4958(8)$ & $4956(4)$ & $7333(3)$ \\
\hline C(99) & $5730(8)$ & $4355(4)$ & $6929(2)$ \\
\hline$C(100)$ & $6868(7)$ & $4526(4)$ & $6969(3)$ \\
\hline$C(101)$ & $8177(7)$ & $5334(4)$ & $7217(3)$ \\
\hline$C(102)$ & $8276(7)$ & $5937(4)$ & $7370(3)$ \\
\hline$C(103)$ & $7478(8)$ & $6516(4)$ & $7753(2)$ \\
\hline$C(104)$ & $6732(8)$ & $6473(4)$ & $8028(2)$ \\
\hline$C(105)$ & $2917(12)$ & 2799(9) & $8039(5)$ \\
\hline$C(106)$ & $1878(14)$ & $2409(10)$ & $7933(5)$ \\
\hline$C(107)$ & $1082(9)$ & $1524(8)$ & $7739(4)$ \\
\hline$C(108)$ & $1256(9)$ & $947(8)$ & $7614(3)$ \\
\hline C(109) & $1935(12)$ & $413(5)$ & $7166(4)$ \\
\hline$C(110)$ & $2474(11)$ & $453(5)$ & $6861(4)$ \\
\hline$C(111)$ & $4118(13)$ & $835(7)$ & $6665(3)$ \\
\hline$C(112)$ & $5152(12)$ & $1146(9)$ & $6782(3)$ \\
\hline$C(113)$ & $6040(10)$ & $2073(11)$ & $7038(4)$ \\
\hline$C(114)$ & $5851(12)$ & $2632(8)$ & $7123(4)$ \\
\hline$C(115)$ & $4978(13)$ & $3211(5)$ & $7489(4)$ \\
\hline$C(116)$ & $4449(12)$ & $3259(6)$ & $7777(5)$ \\
\hline$C(117)$ & $3992(7)$ & $1033(4)$ & $8132(2)$ \\
\hline$C(118)$ & $4990(7)$ & $1457(4)$ & $8244(2)$ \\
\hline C(119) & $6039(7)$ & $2278(4)$ & $8116(2)$ \\
\hline$C(120)$ & $7054(7)$ & 2061(4) & $8099(2)$ \\
\hline$C(121)$ & $8020(7)$ & $1556(5)$ & $7733(3)$ \\
\hline$C(122)$ & $7826(8)$ & $1147(5)$ & $7394(3)$ \\
\hline$C(123)$ & $8640(9)$ & $559(6)$ & $7024(3)$ \\
\hline$C(124)$ & 7997(11) & $-1(5)$ & $7000(3)$ \\
\hline$C(125)$ & $6374(13)$ & $-548(6)$ & $6987(5)$ \\
\hline$C(126)$ & $5455(11)$ & $-542(6)$ & $7072(3)$ \\
\hline$C(127)$ & $4588(11)$ & $18(5)$ & $7447(3)$ \\
\hline $\mathrm{C}(128)$ & $4748(10)$ & $400(5)$ & $7768(3)$ \\
\hline$C(129)$ & $5693(9)$ & $6277(5)$ & $10271(3)$ \\
\hline$C(130)$ & $6675(9)$ & $6538(4)$ & $10151(3)$ \\
\hline
\end{tabular}




\begin{tabular}{|c|c|c|c|}
\hline $\mathrm{C}(131)$ & $7721(7)$ & $7408(5)$ & $10100(3)$ \\
\hline C(132) & $7800(7)$ & $8020(5)$ & $10168(3)$ \\
\hline$C(133)$ & $7009(10)$ & $8758(4)$ & $10054(3)$ \\
\hline$C(134)$ & $6072(9)$ & 8891(4) & 9895(3) \\
\hline$C(135)$ & $4265(9)$ & $8783(5)$ & $9914(3)$ \\
\hline$C(136)$ & $3376(9)$ & $8549(4)$ & $10080(3)$ \\
\hline$C(137)$ & $2305(7)$ & $7689(5)$ & $10138(3)$ \\
\hline C(138) & 2161(7) & $7065(5)$ & $10034(3)$ \\
\hline C(139) & 2951(9) & $6303(4)$ & $10056(2)$ \\
\hline$C(140)$ & $3855(8)$ & $6127(4)$ & $10216(3)$ \\
\hline $\mathrm{C}(141)$ & $3570(6)$ & $7182(3)$ & $9152(2)$ \\
\hline$C(142)$ & 2551(6) & $7314(4)$ & $9050(3)$ \\
\hline$C(143)$ & $3620(7)$ & $6665(3)$ & $9224(2)$ \\
\hline$C(144)$ & $4577(8)$ & $6538(4)$ & $9313(2)$ \\
\hline$C(145)$ & $5474(7)$ & $6942(5)$ & $9321(2)$ \\
\hline$C(146)$ & $5432(7)$ & $7479(5)$ & $9254(2)$ \\
\hline$C(147)$ & $4500(6)$ & $7577(4)$ & $9173(2)$ \\
\hline C(148) & $4653(7)$ & $7465(5)$ & $10935(2)$ \\
\hline C(149) & 4499(8) & $7955(5)$ & $10853(2)$ \\
\hline$C(150)$ & 5319(9) & $8407(5)$ & $10871(2)$ \\
\hline$C(151)$ & $3507(7)$ & $2137(4)$ & 8946(3) \\
\hline$C(152)$ & $2446(8)$ & $2194(5)$ & $8836(4)$ \\
\hline$C(153)$ & $3663(8)$ & $1647(4)$ & $9030(2)$ \\
\hline C(154) & $4367(7)$ & $2580(4)$ & $8960(2)$ \\
\hline$C(155)$ & $2313(8)$ & $5200(5)$ & 4931(3) \\
\hline$C(156)$ & $2324(8)$ & $4329(4)$ & $5073(3)$ \\
\hline$C(157)$ & $2808(7)$ & $3827(4)$ & 4993(3) \\
\hline C(158) & $4424(9)$ & $3540(4)$ & $5050(3)$ \\
\hline$C(159)$ & 5469(9) & $3708(4)$ & $5216(3)$ \\
\hline$C(160)$ & $7055(8)$ & $4341(5)$ & $5226(3)$ \\
\hline C(161) & $6330(7)$ & $5355(4)$ & $6043(2)$ \\
\hline$C(162)$ & $7368(7)$ & $5296(5)$ & $6199(3)$ \\
\hline C(163) & $5458(7)$ & $4922(4)$ & $6027(2)$ \\
\hline C(164) & $4522(9)$ & $4984(5)$ & $5906(3)$ \\
\hline$C(165)$ & $4400(10)$ & $5451(6)$ & $5802(3)$ \\
\hline$C(166)$ & $5316(11)$ & $5881(5)$ & $5824(3)$ \\
\hline$C(167)$ & $6260(9)$ & $5828(4)$ & $5941(2)$ \\
\hline$C(168)$ & $2524(7)$ & 399(4) & $4954(2)$ \\
\hline C(169) & $3194(7)$ & $827(5)$ & $4807(3)$ \\
\hline $\mathrm{C}(170)$ & $4890(8)$ & $1359(4)$ & $4812(2)$ \\
\hline $\mathrm{C}(171)$ & $5948(9)$ & $1476(4)$ & $4982(3)$ \\
\hline$C(172)$ & $7409(7)$ & $1082(4)$ & $5072(3)$ \\
\hline$C(173)$ & $7802(7)$ & $542(4)$ & $4980(3)$ \\
\hline$C(174)$ & $6285(7)$ & $332(4)$ & $5987(2)$ \\
\hline$C(175)$ & $7368(8)$ & $321(6)$ & $6113(3)$ \\
\hline
\end{tabular}




\begin{tabular}{|c|c|c|c|}
\hline C(176) & $6073(8)$ & $792(4)$ & $5894(2)$ \\
\hline$C(177)$ & $5068(10)$ & $798(5)$ & $5778(3)$ \\
\hline$C(178)$ & $4274(9)$ & $337(5)$ & $5770(3)$ \\
\hline$C(179)$ & $4504(7)$ & $-123(4)$ & $5863(2)$ \\
\hline$C(180)$ & $5498(6)$ & $-133(4)$ & $5972(2)$ \\
\hline $\mathrm{Hf}(1)$ & 713(1) & $7870(1)$ & $6515(1)$ \\
\hline $\operatorname{Hf}(2)$ & $9398(1)$ & $9227(1)$ & $8425(1)$ \\
\hline $\mathrm{Hf}(3)$ & $9233(1)$ & $4668(1)$ & $8434(1)$ \\
\hline $\mathrm{Hf}(4)$ & $840(1)$ & $2925(1)$ & $6597(1)$ \\
\hline $\mathrm{K}(1)$ & $4440(1)$ & $6836(1)$ & $7583(1)$ \\
\hline$K(2)$ & $3785(1)$ & $1709(1)$ & $7464(1)$ \\
\hline$K(3)$ & 4998(1) & $7523(1)$ & $10080(1)$ \\
\hline$K(4)$ & 5000 & 5000 & 5000 \\
\hline$K(5)$ & 5000 & 0 & 5000 \\
\hline $\mathrm{N}(1)$ & $-230(7)$ & $8301(4)$ & $6805(2)$ \\
\hline $\mathrm{N}(2)$ & 189(6) & $7094(3)$ & $6625(2)$ \\
\hline $\mathrm{N}(3)$ & $2093(6)$ & $8247(3)$ & $6780(2)$ \\
\hline $\mathrm{N}(4)$ & $8686(6)$ & $9589(3)$ & $8097(2)$ \\
\hline $\mathrm{N}(5)$ & $8958(5)$ & $8385(3)$ & $8232(2)$ \\
\hline $\mathrm{N}(6)$ & $10866(5)$ & $9439(3)$ & $8264(2)$ \\
\hline $\mathrm{N}(7)$ & $7909(5)$ & $4269(3)$ & $8148(2)$ \\
\hline $\mathrm{N}(8)$ & $9689(5)$ & $5470(3)$ & $8356(2)$ \\
\hline $\mathrm{N}(9)$ & $10280(5)$ & $4300(3)$ & $8146(2)$ \\
\hline $\mathrm{N}(10)$ & $1885(5)$ & $3618(3)$ & $6827(2)$ \\
\hline $\mathrm{N}(11)$ & $-450(6)$ & $2975(4)$ & $6872(2)$ \\
\hline $\mathrm{N}(12)$ & $1222(8)$ & $2248(4)$ & $6764(2)$ \\
\hline $\mathrm{O}(1)$ & $2456(4)$ & $6295(2)$ & $7210(1)$ \\
\hline $\mathrm{O}(2)$ & $2397(5)$ & $7028(3)$ & $7802(2)$ \\
\hline $\mathrm{O}(3)$ & $4273(5)$ & $7846(3)$ & $8023(2)$ \\
\hline $\mathrm{O}(4)$ & $5406(5)$ & $7934(3)$ & $7472(2)$ \\
\hline $\mathrm{O}(5)$ & $6011(5)$ & $6954(3)$ & $7129(2)$ \\
\hline $\mathrm{O}(6)$ & $4341(5)$ & $6038(3)$ & $6991(2)$ \\
\hline $\mathrm{O}(7)$ & $6253(4)$ & $6945(2)$ & $8064(1)$ \\
\hline $\mathrm{O}(8)$ & $4171(4)$ & $6486(2)$ & $8161(1)$ \\
\hline $\mathrm{O}(9)$ & $4068(4)$ & $5429(2)$ & $7747(1)$ \\
\hline $\mathrm{O}(10)$ & $5242(5)$ & $4422(3)$ & $7232(2)$ \\
\hline $\mathrm{O}(11)$ & $7156(5)$ & $5138(3)$ & $7074(2)$ \\
\hline $\mathrm{O}(12)$ & $7592(5)$ & $5963(3)$ & $7627(2)$ \\
\hline $\mathrm{O}(13)$ & $3490(8)$ & $2826(4)$ & $7751(3)$ \\
\hline $\mathrm{O}(14)$ & $2057(6)$ & $1872(5)$ & $7825(2)$ \\
\hline $\mathrm{O}(15)$ & $1815(6)$ & $945(3)$ & $7316(2)$ \\
\hline $\mathrm{O}(16)$ & $3517(7)$ & $780(3)$ & $6942(2)$ \\
\hline $\mathrm{O}(17)$ & $5054(6)$ & $1713(4)$ & $6933(2)$ \\
\hline $\mathrm{O}(18)$ & $5265(6)$ & $2704(3)$ & $7419(2)$ \\
\hline $\mathrm{O}(19)$ & $3964(5)$ & $728(3)$ & $7809(2)$ \\
\hline
\end{tabular}




\begin{tabular}{|c|c|c|c|c|}
\hline $\mathrm{O}(20)$ & $5115(4)$ & $1837(2)$ & $8028(1)$ & 40 \\
\hline $\mathrm{O}(21)$ & $7071(5)$ & $1752(3)$ & $7781(2)$ & 51 \\
\hline $\mathrm{O}(22)$ & $8722(5)$ & $924(4)$ & $7344(2)$ & 72 \\
\hline $\mathrm{O}(23)$ & $6944(8)$ & $25(4)$ & $6968(2)$ & 102 \\
\hline $\mathrm{O}(24)$ & $5432(7)$ & $-238(4)$ & $7398(2)$ & 88 \\
\hline $\mathrm{O}(25)$ & $4826(6)$ & $6407(3)$ & $10115(2)$ & 55 \\
\hline $\mathrm{O}(26)$ & $6808(5)$ & $7148(3)$ & $10213(2)$ & 56 \\
\hline $\mathrm{O}(27)$ & $6949(5)$ & $8181(3)$ & $10011(2)$ & 52 \\
\hline $\mathrm{O}(28)$ & $5164(6)$ & $8657(3)$ & $10044(2)$ & 57 \\
\hline $\mathrm{O}(29)$ & $3178(5)$ & $7943(3)$ & $10007(2)$ & 59 \\
\hline $\mathrm{O}(30)$ & $3032(5)$ & $6873(3)$ & $10159(2)$ & 53 \\
\hline $\mathrm{O}(31)$ & $2819(4)$ & $4730(3)$ & 4907(2) & 47 \\
\hline $\mathrm{O}(32)$ & $3882(5)$ & $3979(2)$ & $5122(2)$ & 50 \\
\hline $\mathrm{O}(33)$ & $6074(5)$ & $4156(3)$ & $5070(2)$ & 53 \\
\hline $\mathrm{O}(34)$ & $7147(4)$ & 117(3) & $5112(1)$ & 41 \\
\hline $\mathrm{O}(35)$ & $6387(5)$ & $985(2)$ & $4915(2)$ & 46 \\
\hline$O(36)$ & $4223(5)$ & $942(2)$ & $4947(1)$ & 46 \\
\hline $\operatorname{Si}(1)$ & $1585(2)$ & $7473(1)$ & $5923(1)$ & 26 \\
\hline $\operatorname{Si}(2)$ & $3387(2)$ & $7530(1)$ & $5940(1)$ & 40 \\
\hline $\operatorname{Si}(3)$ & $842(2)$ & $6543(1)$ & $5662(1)$ & 38 \\
\hline $\operatorname{Si}(4)$ & $-201(2)$ & $8255(1)$ & 5991(1) & 26 \\
\hline $\operatorname{Si}(5)$ & 293(2) & $9224(1)$ & $5985(1)$ & 31 \\
\hline $\operatorname{Si}(6)$ & $-2035(2)$ & $8044(1)$ & $5896(1)$ & 36 \\
\hline $\operatorname{Si}(7)$ & $10126(2)$ & $9161(1)$ & $9069(1)$ & 26 \\
\hline $\operatorname{Si}(8)$ & $9728(2)$ & $8245(1)$ & $9174(1)$ & 28 \\
\hline $\operatorname{Si}(9)$ & $11910(2)$ & $9464(1)$ & $9237(1)$ & 36 \\
\hline $\operatorname{Si}(10)$ & $8223(2)$ & $9795(1)$ & 8901(1) & 26 \\
\hline $\operatorname{Si}(11)$ & $6393(2)$ & $9666(1)$ & $8869(1)$ & 34 \\
\hline $\operatorname{Si}(12)$ & $8836(2)$ & $10783(1)$ & $9004(1)$ & 44 \\
\hline $\operatorname{Si}(13)$ & $10081(2)$ & $4228(1)$ & $8951(1)$ & 24 \\
\hline $\operatorname{Si}(14)$ & $9626(2)$ & $3250(1)$ & $8929(1)$ & 29 \\
\hline $\operatorname{Si}(15)$ & $11917(2)$ & $4484(1)$ & $9061(1)$ & 29 \\
\hline $\operatorname{Si}(16)$ & 8261(1) & $4988(1)$ & $9019(1)$ & 22 \\
\hline $\operatorname{Si}(17)$ & $6466(2)$ & 4946(1) & $8987(1)$ & 33 \\
\hline $\operatorname{Si}(18)$ & $9012(2)$ & $5886(1)$ & $9320(1)$ & 30 \\
\hline $\operatorname{Si}(19)$ & $1685(2)$ & $2548(1)$ & $5990(1)$ & 32 \\
\hline $\operatorname{Si}(20)$ & $3474(2)$ & $2612(1)$ & $5935(1)$ & 38 \\
\hline $\operatorname{Si}(21)$ & $992(2)$ & $1606(1)$ & $5750(1)$ & 42 \\
\hline $\operatorname{Si}(22)$ & $-213(2)$ & $3247(1)$ & $6074(1)$ & 34 \\
\hline $\operatorname{Si}(23)$ & $177(2)$ & $4231(1)$ & $6109(1)$ & 38 \\
\hline $\operatorname{Si}(24)$ & $-2033(2)$ & 2989(1) & $5965(1)$ & 46 \\
\hline
\end{tabular}


Table S18. Bond lengths $(\AA)$ and angles $\left({ }^{\circ}\right)$ for 4 .

\begin{tabular}{|c|c|c|c|}
\hline $\mathrm{C}(1)-\mathrm{N}(1)$ & $1.463(12)$ & $\mathrm{C}(42)-\mathrm{N}(7)$ & $1.490(11)$ \\
\hline $\mathrm{C}(2)-\mathrm{N}(1)$ & $1.457(11)$ & $\mathrm{C}(43)-\mathrm{N}(8)$ & $1.472(10)$ \\
\hline $\mathrm{C}(3)-\mathrm{N}(3)$ & $1.488(12)$ & $\mathrm{C}(44)-\mathrm{N}(8)$ & $1.455(10)$ \\
\hline $\mathrm{C}(4)-\mathrm{N}(3)$ & $1.448(11)$ & $\mathrm{C}(45)-\mathrm{N}(9)$ & $1.478(11)$ \\
\hline $\mathrm{C}(5)-\mathrm{N}(2)$ & $1.446(11)$ & C(46)-N(9) & $1.444(11)$ \\
\hline $\mathrm{C}(6)-\mathrm{N}(2)$ & $1.470(11)$ & $\mathrm{C}(47)-\mathrm{C}(48)$ & $1.541(10)$ \\
\hline$C(7)-C(8)$ & $1.544(10)$ & $\mathrm{C}(47)-\mathrm{Si}(13)$ & $1.953(7)$ \\
\hline $\mathrm{C}(7)-\mathrm{Si}(1)$ & $1.945(7)$ & $\mathrm{C}(48)-\mathrm{Si}(16)$ & $1.936(7)$ \\
\hline C(8)-Si(4) & $1.960(8)$ & $\mathrm{C}(49)-\mathrm{Si}(14)$ & $1.873(8)$ \\
\hline $\mathrm{C}(9)-\mathrm{Si}(2)$ & $1.885(10)$ & $\mathrm{C}(50)-\mathrm{Si}(14)$ & $1.871(8)$ \\
\hline $\mathrm{C}(10)-\mathrm{Si}(2)$ & $1.890(9)$ & $\mathrm{C}(51)-\mathrm{Si}(14)$ & $1.906(9)$ \\
\hline $\mathrm{C}(11)-\mathrm{Si}(2)$ & $1.899(10)$ & $\mathrm{C}(52)-\mathrm{Si}(15)$ & $1.896(9)$ \\
\hline $\mathrm{C}(12)-\mathrm{Si}(3)$ & $1.909(9)$ & $\mathrm{C}(53)-\mathrm{Si}(15)$ & $1.891(8)$ \\
\hline $\mathrm{C}(13)-\mathrm{Si}(3)$ & $1.913(9)$ & $\mathrm{C}(54)-\mathrm{Si}(15)$ & $1.893(9)$ \\
\hline C(14)-Si(3) & $1.888(9)$ & $\mathrm{C}(55)-\mathrm{Si}(17)$ & $1.904(9)$ \\
\hline$C(15)-\operatorname{Si}(5)$ & $1.873(8)$ & $\mathrm{C}(56)-\mathrm{Si}(17)$ & $1.901(9)$ \\
\hline$C(16)-\operatorname{Si}(5)$ & $1.878(9)$ & $\mathrm{C}(57)-\mathrm{Si}(17)$ & $1.902(8)$ \\
\hline$C(17)-\operatorname{Si}(5)$ & $1.892(8)$ & $\mathrm{C}(58)-\mathrm{Si}(18)$ & $1.916(8)$ \\
\hline $\mathrm{C}(18)-\mathrm{Si}(6)$ & $1.900(8)$ & C(59)-Si(18) & $1.929(9)$ \\
\hline C(19)-Si(6) & $1.890(9)$ & $\mathrm{C}(60)-\mathrm{Si}(18)$ & $1.908(8)$ \\
\hline $\mathrm{C}(20)-\mathrm{Si}(6)$ & $1.842(9)$ & $\mathrm{C}(61)-\mathrm{N}(10)$ & $1.451(11)$ \\
\hline $\mathrm{C}(21)-\mathrm{N}(4)$ & $1.456(11)$ & $\mathrm{C}(62)-\mathrm{N}(10)$ & $1.524(12)$ \\
\hline $\mathrm{C}(22)-\mathrm{N}(4)$ & $1.488(11)$ & $\mathrm{C}(63)-\mathrm{N}(11)$ & $1.472(13)$ \\
\hline $\mathrm{C}(23)-\mathrm{N}(5)$ & $1.418(10)$ & $\mathrm{C}(64)-\mathrm{N}(11)$ & $1.469(12)$ \\
\hline $\mathrm{C}(24)-\mathrm{N}(5)$ & $1.478(10)$ & $\mathrm{C}(65)-\mathrm{N}(12)$ & $1.355(15)$ \\
\hline $\mathrm{C}(25)-\mathrm{N}(6)$ & $1.448(10)$ & $\mathrm{C}(66)-\mathrm{N}(12)$ & $1.566(16)$ \\
\hline $\mathrm{C}(26)-\mathrm{N}(6)$ & $1.451(11)$ & $\mathrm{C}(67)-\mathrm{C}(68)$ & $1.505(12)$ \\
\hline $\mathrm{C}(27)-\mathrm{C}(28)$ & $1.528(10)$ & $\mathrm{C}(67)-\mathrm{Si}(22)$ & $1.928(8)$ \\
\hline $\mathrm{C}(27)-\mathrm{Si}(7)$ & $1.940(8)$ & $\mathrm{C}(68)-\mathrm{Si}(19)$ & $1.964(9)$ \\
\hline $\mathrm{C}(28)-\mathrm{Si}(10)$ & $1.932(7)$ & $C(69)-\operatorname{Si}(20)$ & $1.918(9)$ \\
\hline C(29)-Si(8) & $1.884(8)$ & $\mathrm{C}(70)-\mathrm{Si}(20)$ & $1.882(9)$ \\
\hline $\mathrm{C}(30)-\mathrm{Si}(8)$ & $1.889(8)$ & $\mathrm{C}(71)-\mathrm{Si}(20)$ & $1.912(9)$ \\
\hline $\mathrm{C}(31)-\mathrm{Si}(8)$ & $1.924(8)$ & $\mathrm{C}(72)-\mathrm{Si}(21)$ & $1.904(10)$ \\
\hline $\mathrm{C}(32)-\mathrm{Si}(9)$ & $1.874(9)$ & $\mathrm{C}(73)-\mathrm{Si}(21)$ & $1.908(9)$ \\
\hline $\mathrm{C}(33)-\mathrm{Si}(9)$ & $1.908(9)$ & $\mathrm{C}(74)-\mathrm{Si}(21)$ & $1.890(9)$ \\
\hline C(34)-Si(9) & $1.872(8)$ & $\mathrm{C}(75)-\mathrm{Si}(23)$ & $1.875(9)$ \\
\hline $\mathrm{C}(35)-\mathrm{Si}(11)$ & $1.907(8)$ & $C(76)-\operatorname{Si}(23)$ & $1.909(9)$ \\
\hline $\mathrm{C}(36)-\mathrm{Si}(11)$ & $1.907(9)$ & $\mathrm{C}(77)-\mathrm{Si}(23)$ & $1.905(9)$ \\
\hline $\mathrm{C}(37)-\mathrm{Si}(11)$ & $1.911(9)$ & $\mathrm{C}(78)-\mathrm{Si}(24)$ & $1.865(10)$ \\
\hline $\mathrm{C}(38)-\mathrm{Si}(12)$ & $1.919(10)$ & $\mathrm{C}(79)-\mathrm{Si}(24)$ & $1.899(11)$ \\
\hline C(39)-Si(12) & $1.896(10)$ & $\mathrm{C}(80)-\mathrm{Si}(24)$ & $1.870(11)$ \\
\hline $\mathrm{C}(40)-\mathrm{Si}(12)$ & $1.861(11)$ & $\mathrm{C}(81)-\mathrm{O}(1)$ & $1.437(10)$ \\
\hline $\mathrm{C}(41)-\mathrm{N}(7)$ & $1.417(12)$ & $\mathrm{C}(81)-\mathrm{C}(82)$ & $1.462(13)$ \\
\hline
\end{tabular}




\begin{tabular}{|c|c|}
\hline $\mathrm{C}(82)-\mathrm{O}(2)$ & $1.413(10)$ \\
\hline $\mathrm{C}(83)-\mathrm{O}(2)$ & $1.438(11)$ \\
\hline $\mathrm{C}(83)-\mathrm{C}(84)$ & $1.468(14)$ \\
\hline $\mathrm{C}(84)-\mathrm{O}(3)$ & $1.446(11)$ \\
\hline $\mathrm{C}(85)-\mathrm{O}(3)$ & $1.447(11)$ \\
\hline $\mathrm{C}(85)-\mathrm{C}(86)$ & $1.482(13)$ \\
\hline $\mathrm{C}(86)-\mathrm{O}(4)$ & $1.414(11)$ \\
\hline $\mathrm{C}(87)-\mathrm{O}(4)$ & $1.414(11)$ \\
\hline $\mathrm{C}(87)-\mathrm{C}(88)$ & $1.502(15)$ \\
\hline $\mathrm{C}(88)-\mathrm{O}(5)$ & $1.443(13)$ \\
\hline C(89)-C(90) & $1.204(16)$ \\
\hline $\mathrm{C}(89)-\mathrm{O}(5)$ & $1.427(13)$ \\
\hline $\mathrm{C}(90)-\mathrm{O}(6)$ & $1.394(11)$ \\
\hline $\mathrm{C}(91)-\mathrm{O}(6)$ & $1.384(11)$ \\
\hline$C(91)-C(92)$ & $1.509(14)$ \\
\hline $\mathrm{C}(92)-\mathrm{O}(1)$ & $1.420(10)$ \\
\hline $\mathrm{C}(93)-\mathrm{O}(7)$ & $1.424(9)$ \\
\hline $\mathrm{C}(93)-\mathrm{C}(94)$ & $1.510(11)$ \\
\hline $\mathrm{C}(94)-\mathrm{O}(8)$ & $1.419(9)$ \\
\hline $\mathrm{C}(95)-\mathrm{O}(8)$ & $1.429(9)$ \\
\hline$C(95)-C(96)$ & $1.531(12)$ \\
\hline $\mathrm{C}(96)-\mathrm{O}(9)$ & $1.411(9)$ \\
\hline $\mathrm{C}(97)-\mathrm{O}(9)$ & $1.411(10)$ \\
\hline $\mathrm{C}(97)-\mathrm{C}(98)$ & $1.540(12)$ \\
\hline $\mathrm{C}(98)-\mathrm{O}(10)$ & $1.434(10)$ \\
\hline $\mathrm{C}(99)-\mathrm{O}(10)$ & $1.432(10)$ \\
\hline$C(99)-C(100)$ & $1.463(13)$ \\
\hline $\mathrm{C}(100)-\mathrm{O}(11)$ & $1.462(11)$ \\
\hline $\mathrm{C}(101)-\mathrm{O}(11)$ & $1.402(11)$ \\
\hline $\mathrm{C}(101)-\mathrm{C}(102)$ & $1.483(13)$ \\
\hline $\mathrm{C}(102)-\mathrm{O}(12)$ & $1.423(10)$ \\
\hline $\mathrm{C}(103)-\mathrm{O}(12)$ & $1.407(10)$ \\
\hline$C(103)-C(104)$ & $1.539(11)$ \\
\hline $\mathrm{C}(104)-\mathrm{O}(7)$ & $1.415(10)$ \\
\hline $\mathrm{C}(105)-\mathrm{O}(13)$ & $1.44(2)$ \\
\hline$C(105)-C(106)$ & $1.50(2)$ \\
\hline $\mathrm{C}(106)-\mathrm{O}(14)$ & $1.39(2)$ \\
\hline $\mathrm{C}(107)-\mathrm{O}(14)$ & $1.386(15)$ \\
\hline $\mathrm{C}(107)-\mathrm{C}(108)$ & $1.49(2)$ \\
\hline $\mathrm{C}(108)-\mathrm{O}(15)$ & $1.466(15)$ \\
\hline $\mathrm{C}(109)-\mathrm{O}(15)$ & $1.386(16)$ \\
\hline$C(109)-C(110)$ & $1.49(2)$ \\
\hline $\mathrm{C}(110)-\mathrm{O}(16)$ & $1.438(15)$ \\
\hline $\mathrm{C}(111)-\mathrm{O}(16)$ & $1.433(16)$ \\
\hline$C(111)-C(112)$ & $1.44(2)$ \\
\hline
\end{tabular}

\begin{tabular}{|c|c|}
\hline $\mathrm{C}(112)-\mathrm{O}(17)$ & $1.457(18)$ \\
\hline$C(113)-C(114)$ & $1.43(3)$ \\
\hline $\mathrm{C}(113)-\mathrm{O}(17)$ & $1.420(18)$ \\
\hline $\mathrm{C}(114)-\mathrm{O}(18)$ & $1.477(19)$ \\
\hline $\mathrm{C}(115)-\mathrm{O}(18)$ & $1.359(18)$ \\
\hline$C(115)-C(116)$ & $1.40(2)$ \\
\hline $\mathrm{C}(116)-\mathrm{O}(13)$ & $1.467(18)$ \\
\hline $\mathrm{C}(117)-\mathrm{O}(19)$ & $1.411(11)$ \\
\hline$C(117)-C(118)$ & $1.504(12)$ \\
\hline $\mathrm{C}(118)-\mathrm{O}(20)$ & $1.410(10)$ \\
\hline $\mathrm{C}(119)-\mathrm{O}(20)$ & $1.445(10)$ \\
\hline$C(119)-C(120)$ & $1.529(12)$ \\
\hline $\mathrm{C}(120)-\mathrm{O}(21)$ & $1.406(10)$ \\
\hline $\mathrm{C}(121)-\mathrm{O}(21)$ & $1.428(10)$ \\
\hline $\mathrm{C}(121)-\mathrm{C}(122)$ & $1.557(15)$ \\
\hline $\mathrm{C}(122)-\mathrm{O}(22)$ & $1.400(11)$ \\
\hline $\mathrm{C}(123)-\mathrm{O}(22)$ & $1.450(13)$ \\
\hline$C(123)-C(124)$ & $1.454(17)$ \\
\hline $\mathrm{C}(124)-\mathrm{O}(23)$ & $1.399(15)$ \\
\hline$C(125)-C(126)$ & $1.269(17)$ \\
\hline $\mathrm{C}(125)-\mathrm{O}(23)$ & $1.482(16)$ \\
\hline $\mathrm{C}(126)-\mathrm{O}(24)$ & $1.429(14)$ \\
\hline $\mathrm{C}(127)-\mathrm{O}(24)$ & $1.383(14)$ \\
\hline $\mathrm{C}(127)-\mathrm{C}(128)$ & $1.467(15)$ \\
\hline $\mathrm{C}(128)-\mathrm{O}(19)$ & $1.425(12)$ \\
\hline $\mathrm{C}(129)-\mathrm{O}(25)$ & $1.412(12)$ \\
\hline$C(129)-C(130)$ & $1.472(15)$ \\
\hline $\mathrm{C}(130)-\mathrm{O}(26)$ & $1.454(12)$ \\
\hline $\mathrm{C}(131)-\mathrm{O}(26)$ & $1.384(12)$ \\
\hline $\mathrm{C}(131)-\mathrm{C}(132)$ & $1.467(15)$ \\
\hline $\mathrm{C}(132)-\mathrm{O}(27)$ & $1.433(11)$ \\
\hline $\mathrm{C}(133)-\mathrm{O}(27)$ & $1.390(12)$ \\
\hline $\mathrm{C}(133)-\mathrm{C}(134)$ & $1.500(16)$ \\
\hline $\mathrm{C}(134)-\mathrm{O}(28)$ & $1.431(11)$ \\
\hline $\mathrm{C}(135)-\mathrm{O}(28)$ & $1.396(12)$ \\
\hline$C(135)-C(136)$ & $1.454(15)$ \\
\hline $\mathrm{C}(136)-\mathrm{O}(29)$ & $1.439(12)$ \\
\hline $\mathrm{C}(137)-\mathrm{O}(29)$ & $1.376(11)$ \\
\hline $\mathrm{C}(137)-\mathrm{C}(138)$ & $1.492(14)$ \\
\hline $\mathrm{C}(138)-\mathrm{O}(30)$ & $1.438(11)$ \\
\hline C(139)-O(30) & $1.375(11)$ \\
\hline $\mathrm{C}(139)-\mathrm{C}(140)$ & $1.518(14)$ \\
\hline $\mathrm{C}(140)-\mathrm{O}(25)$ & $1.440(11)$ \\
\hline$C(141)-C(143)$ & $1.381(11)$ \\
\hline$C(141)-C(147)$ & $1.392(11)$ \\
\hline
\end{tabular}




\begin{tabular}{|c|c|c|c|}
\hline$C(141)-C(142)$ & $1.503(11)$ & $C(174)-C(180)$ & $1.373(12)$ \\
\hline$C(143)-C(144)$ & $1.405(12)$ & $C(174)-C(175)$ & $1.505(13)$ \\
\hline $\mathrm{C}(144)-\mathrm{C}(145)$ & $1.377(15)$ & $C(176)-C(177)$ & $1.391(15)$ \\
\hline $\mathrm{C}(145)-\mathrm{C}(146)$ & $1.419(15)$ & $\mathrm{C}(177)-\mathrm{C}(178)$ & $1.377(15)$ \\
\hline $\mathrm{C}(145)-\mathrm{K}(3)$ & $3.352(9)$ & $C(177)-K(5)$ & $3.455(11)$ \\
\hline$C(146)-C(147)$ & $1.338(13)$ & $\mathrm{C}(178)-\mathrm{C}(179)$ & $1.352(14)$ \\
\hline$C(146)-K(3)$ & $3.494(10)$ & $\mathrm{C}(178)-\mathrm{K}(5)$ & $3.381(10)$ \\
\hline $\mathrm{C}(148)-\mathrm{C}(154) \# 1$ & $1.375(13)$ & $\mathrm{C}(179)-\mathrm{C}(180)$ & $1.372(12)$ \\
\hline C(148)-C(149) & $1.367(15)$ & $\mathrm{Hf}(1)-\mathrm{N}(3)$ & $2.043(7)$ \\
\hline$C(149)-C(150)$ & $1.376(15)$ & $\mathrm{Hf}(1)-\mathrm{N}(2)$ & $2.047(7)$ \\
\hline $\mathrm{C}(149)-\mathrm{K}(3)$ & $3.331(10)$ & $\mathrm{Hf}(1)-\mathrm{N}(1)$ & $2.063(7)$ \\
\hline $\mathrm{C}(150)-\mathrm{C}(153) \# 1$ & $1.423(14)$ & $\mathrm{Hf}(1)-\mathrm{Si}(1)$ & $2.846(2)$ \\
\hline$C(151)-C(153)$ & $1.373(13)$ & $\mathrm{Hf}(1)-\mathrm{Si}(4)$ & $2.863(2)$ \\
\hline$C(151)-C(154)$ & $1.398(12)$ & $\mathrm{Hf}(2)-\mathrm{N}(5)$ & $2.043(6)$ \\
\hline $\mathrm{C}(151)-\mathrm{C}(152)$ & $1.492(14)$ & $\mathrm{Hf}(2)-\mathrm{N}(6)$ & $2.062(6)$ \\
\hline $\mathrm{C}(153)-\mathrm{C}(150) \# 1$ & $1.423(14)$ & $\mathrm{Hf}(2)-\mathrm{N}(4)$ & $2.064(7)$ \\
\hline $\mathrm{C}(154)-\mathrm{C}(148) \# 1$ & $1.375(13)$ & $\mathrm{Hf}(2)-\mathrm{Si}(7)$ & $2.864(3)$ \\
\hline $\mathrm{C}(155)-\mathrm{O}(31)$ & $1.438(11)$ & $\mathrm{Hf}(2)-\mathrm{Si}(10)$ & $2.879(2)$ \\
\hline $\mathrm{C}(155)-\mathrm{C}(160) \# 2$ & $1.529(15)$ & $\mathrm{Hf}(3)-\mathrm{N}(8)$ & $2.042(6)$ \\
\hline $\mathrm{C}(156)-\mathrm{O}(31)$ & $1.383(11)$ & $\mathrm{Hf}(3)-\mathrm{N}(7)$ & $2.038(6)$ \\
\hline$C(156)-C(157)$ & $1.492(14)$ & $\mathrm{Hf}(3)-\mathrm{N}(9)$ & $2.085(6)$ \\
\hline $\mathrm{C}(157)-\mathrm{O}(32)$ & $1.442(11)$ & $\mathrm{Hf}(3)-\mathrm{Si}(16)$ & $2.841(2)$ \\
\hline $\mathrm{C}(158)-\mathrm{O}(32)$ & $1.402(12)$ & $\mathrm{Hf}(3)-\mathrm{Si}(13)$ & $2.875(2)$ \\
\hline $\mathrm{C}(158)-\mathrm{C}(159)$ & $1.460(15)$ & $\mathrm{Hf}(4)-\mathrm{N}(10)$ & $2.023(7)$ \\
\hline $\mathrm{C}(159)-\mathrm{O}(33)$ & $1.472(11)$ & $\mathrm{Hf}(4)-\mathrm{N}(12)$ & $2.067(8)$ \\
\hline $\mathrm{C}(160)-\mathrm{O}(33)$ & $1.373(11)$ & $\mathrm{Hf}(4)-\mathrm{N}(11)$ & $2.084(7)$ \\
\hline $\mathrm{C}(160)-\mathrm{C}(155) \# 2$ & $1.529(15)$ & $\mathrm{Hf}(4)-\mathrm{Si}(22)$ & $2.864(2)$ \\
\hline C(161)-C(167) & $1.335(12)$ & Hf(4)-Si(19) & $2.866(2)$ \\
\hline$C(161)-C(163)$ & $1.392(12)$ & $\mathrm{K}(1)-\mathrm{O}(8)$ & $2.722(6)$ \\
\hline $\mathrm{C}(161)-\mathrm{C}(162)$ & $1.533(13)$ & $\mathrm{K}(1)-\mathrm{O}(6)$ & $2.837(6)$ \\
\hline $\mathrm{C}(163)-\mathrm{C}(164)$ & $1.358(14)$ & $\mathrm{K}(1)-\mathrm{O}(5)$ & $2.853(7)$ \\
\hline$C(164)-C(165)$ & $1.341(17)$ & $\mathrm{K}(1)-\mathrm{O}(3)$ & $2.855(6)$ \\
\hline$C(165)-C(166)$ & $1.425(17)$ & $\mathrm{K}(1)-\mathrm{O}(4)$ & $2.885(6)$ \\
\hline $\mathrm{C}(165)-\mathrm{K}(4)$ & $3.489(11)$ & $\mathrm{K}(1)-\mathrm{O}(1)$ & $2.925(6)$ \\
\hline C(166)-C(167) & $1.354(16)$ & $\mathrm{K}(1)-\mathrm{O}(2)$ & $2.955(6)$ \\
\hline $\mathrm{C}(168)-\mathrm{O}(34) \# 3$ & $1.409(10)$ & $\mathrm{K}(1)-\mathrm{O}(7)$ & $2.998(6)$ \\
\hline C(168)-C(169) & $1.477(13)$ & $\mathrm{K}(2)-\mathrm{O}(16)$ & $2.805(7)$ \\
\hline $\mathrm{C}(169)-\mathrm{O}(36)$ & $1.407(11)$ & $\mathrm{K}(2)-\mathrm{O}(20)$ & $2.812(6)$ \\
\hline $\mathrm{C}(170)-\mathrm{O}(36)$ & $1.428(11)$ & $\mathrm{K}(2)-\mathrm{O}(17)$ & $2.813(7)$ \\
\hline$C(170)-C(171)$ & $1.487(14)$ & $\mathrm{K}(2)-\mathrm{O}(14)$ & $2.816(8)$ \\
\hline $\mathrm{C}(171)-\mathrm{O}(35)$ & $1.428(11)$ & $\mathrm{K}(2)-\mathrm{O}(15)$ & $2.853(7)$ \\
\hline $\mathrm{C}(172)-\mathrm{O}(35)$ & $1.427(11)$ & $\mathrm{K}(2)-\mathrm{O}(18)$ & $2.857(7)$ \\
\hline $\mathrm{C}(172)-\mathrm{C}(173)$ & $1.513(14)$ & $\mathrm{K}(2)-\mathrm{O}(13)$ & $2.901(8)$ \\
\hline $\mathrm{C}(173)-\mathrm{O}(34)$ & $1.424(10)$ & $\mathrm{K}(2)-\mathrm{O}(19)$ & $3.084(6)$ \\
\hline$C(174)-C(176)$ & $1.345(13)$ & $\mathrm{K}(3)-\mathrm{O}(25)$ & $2.754(7)$ \\
\hline
\end{tabular}




\begin{tabular}{|c|c|c|c|}
\hline $\mathrm{K}(3)-\mathrm{O}(26)$ & $2.788(7)$ & $\mathrm{K}(5)-\mathrm{C}(177) \# 3$ & $3.455(11)$ \\
\hline $\mathrm{K}(3)-\mathrm{O}(28)$ & $2.798(6)$ & $\mathrm{O}(34)-\mathrm{C}(168) \# 3$ & $1.409(10)$ \\
\hline $\mathrm{K}(3)-\mathrm{O}(27)$ & $2.800(6)$ & $\operatorname{Si}(1)-\operatorname{Si}(2)$ & $2.337(3)$ \\
\hline $\mathrm{K}(3)-\mathrm{O}(29)$ & $2.814(7)$ & $\operatorname{Si}(1)-\operatorname{Si}(3)$ & $2.347(3)$ \\
\hline $\mathrm{K}(3)-\mathrm{O}(30)$ & $2.821(6)$ & $\operatorname{Si}(4)-\operatorname{Si}(5)$ & $2.357(3)$ \\
\hline $\mathrm{K}(4)-\mathrm{O}(33)$ & $2.789(6)$ & $\operatorname{Si}(4)-\operatorname{Si}(6)$ & $2.362(3)$ \\
\hline $\mathrm{K}(4)-\mathrm{O}(33) \# 2$ & $2.789(6)$ & $\operatorname{Si}(7)-\operatorname{Si}(8)$ & $2.347(3)$ \\
\hline $\mathrm{K}(4)-\mathrm{O}(32)$ & $2.800(6)$ & $\operatorname{Si}(7)-\operatorname{Si}(9)$ & $2.350(3)$ \\
\hline $\mathrm{K}(4)-\mathrm{O}(32) \# 2$ & $2.800(6)$ & $\operatorname{Si}(10)-\operatorname{Si}(11)$ & $2.353(3)$ \\
\hline $\mathrm{K}(4)-\mathrm{O}(31) \# 2$ & $2.802(6)$ & $\operatorname{Si}(10)-\operatorname{Si}(12)$ & $2.364(3)$ \\
\hline $\mathrm{K}(4)-\mathrm{O}(31)$ & $2.802(6)$ & $\operatorname{Si}(13)-\operatorname{Si}(14)$ & $2.356(3)$ \\
\hline $\mathrm{K}(4)-\mathrm{C}(165) \# 2$ & $3.489(11)$ & $\operatorname{Si}(13)-\operatorname{Si}(15)$ & $2.372(3)$ \\
\hline $\mathrm{K}(5)-\mathrm{O}(36)$ & $2.760(6)$ & $\operatorname{Si}(16)-\operatorname{Si}(17)$ & $2.334(3)$ \\
\hline $\mathrm{K}(5)-\mathrm{O}(36) \# 3$ & $2.760(6)$ & $\operatorname{Si}(16)-\operatorname{Si}(18)$ & $2.341(3)$ \\
\hline $\mathrm{K}(5)-\mathrm{O}(34) \# 3$ & $2.787(6)$ & $\operatorname{Si}(19)-\operatorname{Si}(21)$ & $2.338(4)$ \\
\hline $\mathrm{K}(5)-\mathrm{O}(34)$ & $2.787(6)$ & $\operatorname{Si}(19)-\operatorname{Si}(20)$ & $2.342(3)$ \\
\hline $\mathrm{K}(5)-\mathrm{O}(35) \# 3$ & $2.823(6)$ & $\operatorname{Si}(22)-\operatorname{Si}(24)$ & $2.350(3)$ \\
\hline $\mathrm{K}(5)-\mathrm{O}(35)$ & $2.823(6)$ & $\operatorname{Si}(22)-\operatorname{Si}(23)$ & $2.357(4)$ \\
\hline $\mathrm{K}(5)-\mathrm{C}(178) \# 3$ & $3.381(10)$ & & \\
\hline $\mathrm{C}(8)-\mathrm{C}(7)-\mathrm{Si}(1)$ & $108.4(5)$ & $\mathrm{O}(10)-\mathrm{C}(98)-\mathrm{C}(97)$ & $105.6(8)$ \\
\hline $\mathrm{C}(7)-\mathrm{C}(8)-\mathrm{Si}(4)$ & $111.1(5)$ & $\mathrm{O}(10)-\mathrm{C}(99)-\mathrm{C}(100)$ & $113.3(8)$ \\
\hline $\mathrm{C}(28)-\mathrm{C}(27)-\mathrm{Si}(7)$ & $110.8(5)$ & $\mathrm{O}(11)-\mathrm{C}(100)-\mathrm{C}(99)$ & $108.8(8)$ \\
\hline$C(27)-C(28)-S i(10)$ & $111.9(5)$ & $\mathrm{O}(11)-\mathrm{C}(101)-\mathrm{C}(102)$ & $107.0(8)$ \\
\hline $\mathrm{C}(48)-\mathrm{C}(47)-\mathrm{Si}(13)$ & $109.2(5)$ & $\mathrm{O}(12)-\mathrm{C}(102)-\mathrm{C}(101)$ & $107.4(7)$ \\
\hline $\mathrm{C}(47)-\mathrm{C}(48)-\mathrm{Si}(16)$ & $109.8(5)$ & $\mathrm{O}(12)-\mathrm{C}(103)-\mathrm{C}(104)$ & $106.1(7)$ \\
\hline $\mathrm{C}(68)-\mathrm{C}(67)-\mathrm{Si}(22)$ & $112.8(6)$ & $\mathrm{O}(7)-\mathrm{C}(104)-\mathrm{C}(103)$ & $107.5(7)$ \\
\hline $\mathrm{C}(67)-\mathrm{C}(68)-\mathrm{Si}(19)$ & $108.3(6)$ & $\mathrm{O}(13)-\mathrm{C}(105)-\mathrm{C}(106)$ & $107.6(13)$ \\
\hline $\mathrm{O}(1)-\mathrm{C}(81)-\mathrm{C}(82)$ & $110.9(7)$ & $\mathrm{O}(14)-\mathrm{C}(106)-\mathrm{C}(105)$ & $107.8(13)$ \\
\hline $\mathrm{O}(2)-\mathrm{C}(82)-\mathrm{C}(81)$ & $108.6(7)$ & $\mathrm{O}(14)-\mathrm{C}(107)-\mathrm{C}(108)$ & $106.9(12)$ \\
\hline $\mathrm{O}(2)-\mathrm{C}(83)-\mathrm{C}(84)$ & $110.0(8)$ & $\mathrm{O}(15)-\mathrm{C}(108)-\mathrm{C}(107)$ & $109.1(10)$ \\
\hline $\mathrm{O}(3)-\mathrm{C}(84)-\mathrm{C}(83)$ & $111.4(8)$ & $\mathrm{O}(15)-\mathrm{C}(109)-\mathrm{C}(110)$ & $109.1(10)$ \\
\hline $\mathrm{O}(3)-\mathrm{C}(85)-\mathrm{C}(86)$ & $108.5(8)$ & $\mathrm{O}(16)-\mathrm{C}(110)-\mathrm{C}(109)$ & $109.5(10)$ \\
\hline $\mathrm{O}(4)-\mathrm{C}(86)-\mathrm{C}(85)$ & $107.2(8)$ & $\mathrm{O}(16)-\mathrm{C}(111)-\mathrm{C}(112)$ & $108.3(11)$ \\
\hline $\mathrm{O}(4)-\mathrm{C}(87)-\mathrm{C}(88)$ & $108.5(8)$ & $\mathrm{C}(111)-\mathrm{C}(112)-\mathrm{O}(17)$ & $106.8(10)$ \\
\hline $\mathrm{O}(5)-\mathrm{C}(88)-\mathrm{C}(87)$ & $108.7(8)$ & $\mathrm{C}(114)-\mathrm{C}(113)-\mathrm{O}(17)$ & $106.3(13)$ \\
\hline $\mathrm{C}(90)-\mathrm{C}(89)-\mathrm{O}(5)$ & $124.3(11)$ & $\mathrm{C}(113)-\mathrm{C}(114)-\mathrm{O}(18)$ & $111.6(11)$ \\
\hline $\mathrm{C}(89)-\mathrm{C}(90)-\mathrm{O}(6)$ & $123.5(11)$ & $\mathrm{O}(18)-\mathrm{C}(115)-\mathrm{C}(116)$ & $110.7(12)$ \\
\hline $\mathrm{O}(6)-\mathrm{C}(91)-\mathrm{C}(92)$ & $109.0(8)$ & $\mathrm{C}(115)-\mathrm{C}(116)-\mathrm{O}(13)$ & $112.7(12)$ \\
\hline $\mathrm{O}(1)-\mathrm{C}(92)-\mathrm{C}(91)$ & $107.4(7)$ & $\mathrm{O}(19)-\mathrm{C}(117)-\mathrm{C}(118)$ & $114.3(8)$ \\
\hline $\mathrm{O}(7)-\mathrm{C}(93)-\mathrm{C}(94)$ & $113.5(7)$ & $\mathrm{O}(20)-\mathrm{C}(118)-\mathrm{C}(117)$ & $107.3(7)$ \\
\hline $\mathrm{O}(8)-\mathrm{C}(94)-\mathrm{C}(93)$ & $107.0(7)$ & $\mathrm{O}(20)-\mathrm{C}(119)-\mathrm{C}(120)$ & $113.5(7)$ \\
\hline $\mathrm{O}(8)-\mathrm{C}(95)-\mathrm{C}(96)$ & $112.7(7)$ & $\mathrm{O}(21)-\mathrm{C}(120)-\mathrm{C}(119)$ & $107.6(7)$ \\
\hline $\mathrm{O}(9)-\mathrm{C}(96)-\mathrm{C}(95)$ & $108.5(7)$ & $\mathrm{O}(21)-\mathrm{C}(121)-\mathrm{C}(122)$ & $104.7(7)$ \\
\hline $\mathrm{O}(9)-\mathrm{C}(97)-\mathrm{C}(98)$ & $106.8(7)$ & $\mathrm{O}(22)-\mathrm{C}(122)-\mathrm{C}(121)$ & $106.7(9)$ \\
\hline
\end{tabular}




$\begin{array}{lc}\mathrm{O}(22)-\mathrm{C}(123)-\mathrm{C}(124) & 114.9(11) \\ \mathrm{O}(23)-\mathrm{C}(124)-\mathrm{C}(123) & 109.5(11) \\ \mathrm{C}(126)-\mathrm{C}(125)-\mathrm{O}(23) & 111.2(14) \\ \mathrm{C}(125)-\mathrm{C}(126)-\mathrm{O}(24) & 112.9(13) \\ \mathrm{O}(24)-\mathrm{C}(127)-\mathrm{C}(128) & 109.3(10) \\ \mathrm{O}(19)-\mathrm{C}(128)-\mathrm{C}(127) & 110.9(9) \\ \mathrm{O}(25)-\mathrm{C}(129)-\mathrm{C}(130) & 111.1(9) \\ \mathrm{O}(26)-\mathrm{C}(130)-\mathrm{C}(129) & 110.9(8) \\ \mathrm{O}(26)-\mathrm{C}(131)-\mathrm{C}(132) & 109.5(8) \\ \mathrm{O}(27)-\mathrm{C}(132)-\mathrm{C}(131) & 112.5(8) \\ \mathrm{O}(27)-\mathrm{C}(133)-\mathrm{C}(134) & 111.7(8) \\ \mathrm{O}(28)-\mathrm{C}(134)-\mathrm{C}(133) & 108.6(8) \\ \mathrm{O}(28)-\mathrm{C}(135)-\mathrm{C}(136) & 109.1(8) \\ \mathrm{O}(29)-\mathrm{C}(136)-\mathrm{C}(135) & 110.6(9) \\ \mathrm{O}(29)-\mathrm{C}(137)-\mathrm{C}(138) & 109.0(8) \\ \mathrm{O}(30)-\mathrm{C}(138)-\mathrm{C}(137) & 110.6(8) \\ \mathrm{O}(30)-\mathrm{C}(139)-\mathrm{C}(140) & 109.0(8) \\ \mathrm{O}(25)-\mathrm{C}(140)-\mathrm{C}(139) & 109.8(8) \\ \mathrm{C}(143)-\mathrm{C}(141)-\mathrm{C}(147) & 117.7(8) \\ \mathrm{C}(143)-\mathrm{C}(141)-\mathrm{C}(142) & 121.4(8) \\ \mathrm{C}(147)-\mathrm{C}(141)-\mathrm{C}(142) & 120.9(8) \\ \mathrm{C}(141)-\mathrm{C}(143)-\mathrm{C}(144) & 121.3(9) \\ \mathrm{C}(145)-\mathrm{C}(144)-\mathrm{C}(143) & 118.4(9) \\ \mathrm{C}(144)-\mathrm{C}(145)-\mathrm{C}(146) & 120.9(9) \\ \mathrm{C}(144)-\mathrm{C}(145)-\mathrm{K}(3) & 88.4(5) \\ \mathrm{C}(146)-\mathrm{C}(145)-\mathrm{K}(3) & 83.7(6) \\ \mathrm{C}(147)-\mathrm{C}(146)-\mathrm{C}(145) & 118.1(9) \\ \mathrm{C}(147)-\mathrm{C}(146)-\mathrm{K}(3) & 95.1(6) \\ \mathrm{C}(145)-\mathrm{C}(146)-\mathrm{K}(3) & 72.5(5) \\ \mathrm{C}(146)-\mathrm{C}(147)-\mathrm{C}(141) & 123.5(9) \\ \mathrm{C}(154) \# 1-\mathrm{C}(148)-\mathrm{C}(149) & 120.1(10) \\ \mathrm{C}(148)-\mathrm{C}(149)-\mathrm{C}(150) & 120.8(10) \\ \mathrm{C}(148)-\mathrm{C}(149)-\mathrm{K}(3) & 91.6(6) \\ \mathrm{C}(150)-\mathrm{C}(149)-\mathrm{K}(3) & 88.0(6) \\ \mathrm{C}(149)-\mathrm{C}(150)-\mathrm{C}(153) \# 1 & 119.0(10) \\ \mathrm{C}(153)-\mathrm{C}(151)-\mathrm{C}(154) & 118.6(9) \\ \mathrm{C}(153)-\mathrm{C}(151)-\mathrm{C}(152) & 120.9(9) \\ \mathrm{C}(154)-\mathrm{C}(151)-\mathrm{C}(152) & 120.5(9) \\ \mathrm{C}(151)-\mathrm{C}(153)-\mathrm{C}(150) \# 1 & 120.3(10) \\ \mathrm{C}(148) \# 1-\mathrm{C}(154)-\mathrm{C}(151) & 121.1(10) \\ \mathrm{O}(31)-\mathrm{C}(155)-\mathrm{C}(160) \# 2 & 110.4(8) \\ \mathrm{O}(31)-\mathrm{C}(156)-\mathrm{C}(157) & 107.7(8) \\ \mathrm{O}(32)-\mathrm{C}(157)-\mathrm{C}(156) & 109.0(8) \\ \mathrm{O}(32)-\mathrm{C}(158)-\mathrm{C}(159) & 110.4(8) \\ \mathrm{C}(158)-\mathrm{C}(159)-\mathrm{O}(33) & 108.3(8) \\ & \end{array}$

\begin{tabular}{|c|c|}
\hline $\mathrm{O}(33)-\mathrm{C}(160)-\mathrm{C}(155) \# 2$ & $110.1(9)$ \\
\hline$C(167)-C(161)-C(163)$ & $120.9(10)$ \\
\hline$C(167)-C(161)-C(162)$ & $120.0(9)$ \\
\hline$C(163)-C(161)-C(162)$ & $119.0(9)$ \\
\hline$C(164)-C(163)-C(161)$ & $119.4(11)$ \\
\hline$C(163)-C(164)-C(165)$ & $122.4(12)$ \\
\hline$C(164)-C(165)-C(166)$ & $116.0(11)$ \\
\hline $\mathrm{C}(164)-\mathrm{C}(165)-\mathrm{K}(4)$ & $95.5(7)$ \\
\hline$C(166)-C(165)-K(4)$ & $85.7(7)$ \\
\hline$C(167)-C(166)-C(165)$ & $122.8(11)$ \\
\hline$C(161)-C(167)-C(166)$ & $118.5(11)$ \\
\hline $\mathrm{O}(34) \# 3-\mathrm{C}(168)-\mathrm{C}(169)$ & $111.0(7)$ \\
\hline $\mathrm{O}(36)-\mathrm{C}(169)-\mathrm{C}(168)$ & $110.3(8)$ \\
\hline $\mathrm{O}(36)-\mathrm{C}(170)-\mathrm{C}(171)$ & $108.6(8)$ \\
\hline $\mathrm{O}(35)-\mathrm{C}(171)-\mathrm{C}(170)$ & $110.1(8)$ \\
\hline $\mathrm{O}(35)-\mathrm{C}(172)-\mathrm{C}(173)$ & $107.1(8)$ \\
\hline $\mathrm{O}(34)-\mathrm{C}(173)-\mathrm{C}(172)$ & $108.1(7)$ \\
\hline$C(176)-C(174)-C(180)$ & $119.5(9)$ \\
\hline $\mathrm{C}(176)-\mathrm{C}(174)-\mathrm{C}(175)$ & $121.0(9)$ \\
\hline $\mathrm{C}(180)-\mathrm{C}(174)-\mathrm{C}(175)$ & $119.5(9)$ \\
\hline $\mathrm{C}(174)-\mathrm{C}(176)-\mathrm{C}(177)$ & $120.5(9)$ \\
\hline $\mathrm{C}(178)-\mathrm{C}(177)-\mathrm{C}(176)$ & $120.0(10)$ \\
\hline $\mathrm{C}(178)-\mathrm{C}(177)-\mathrm{K}(5)$ & $75.4(6)$ \\
\hline $\mathrm{C}(176)-\mathrm{C}(177)-\mathrm{K}(5)$ & $99.9(6)$ \\
\hline $\mathrm{C}(179)-\mathrm{C}(178)-\mathrm{C}(177)$ & $118.5(10)$ \\
\hline $\mathrm{C}(179)-\mathrm{C}(178)-\mathrm{K}(5)$ & $94.4(6)$ \\
\hline $\mathrm{C}(177)-\mathrm{C}(178)-\mathrm{K}(5)$ & $81.4(6)$ \\
\hline $\mathrm{C}(178)-\mathrm{C}(179)-\mathrm{C}(180)$ & $121.6(9)$ \\
\hline $\mathrm{C}(179)-\mathrm{C}(180)-\mathrm{C}(174)$ & $119.8(9)$ \\
\hline $\mathrm{N}(3)-\mathrm{Hf}(1)-\mathrm{N}(2)$ & $109.4(3)$ \\
\hline N(3)-Hf(1)-N(1) & $97.2(3)$ \\
\hline $\mathrm{N}(2)-\mathrm{Hf}(1)-\mathrm{N}(1)$ & $97.9(3)$ \\
\hline N(3)-Hf(1)-Si(1) & $96.8(2)$ \\
\hline N(2)-Hf(1)-Si(1) & $96.1(2)$ \\
\hline N(1)-Hf(1)-Si(1) & $155.7(2)$ \\
\hline N(3)-Hf(1)-Si(4) & $126.3(2)$ \\
\hline $\mathrm{N}(2)-\mathrm{Hf}(1)-\mathrm{Si}(4)$ & $123.5(2)$ \\
\hline N(1)-Hf(1)-Si(4) & $84.9(2)$ \\
\hline $\mathrm{Si}(1)-\mathrm{Hf}(1)-\mathrm{Si}(4)$ & $70.78(7)$ \\
\hline $\mathrm{N}(5)-\mathrm{Hf}(2)-\mathrm{N}(6)$ & $103.0(3)$ \\
\hline $\mathrm{N}(5)-\mathrm{Hf}(2)-\mathrm{N}(4)$ & $103.0(3)$ \\
\hline $\mathrm{N}(6)-\mathrm{Hf}(2)-\mathrm{N}(4)$ & $94.6(3)$ \\
\hline $\mathrm{N}(5)-\mathrm{Hf}(2)-\mathrm{Si}(7)$ & $98.9(2)$ \\
\hline $\mathrm{N}(6)-\mathrm{Hf}(2)-\mathrm{Si}(7)$ & $95.1(2)$ \\
\hline N(4)-Hf(2)-Si(7) & $153.3(2)$ \\
\hline
\end{tabular}




\begin{tabular}{|c|c|c|c|}
\hline $\mathrm{N}(5)-\mathrm{Hf}(2)-\mathrm{Si}(10)$ & $122.1(2)$ & $\mathrm{O}(8)-\mathrm{K}(1)-\mathrm{O}(7)$ & $58.72(15)$ \\
\hline $\mathrm{N}(6)-\mathrm{Hf}(2)-\mathrm{Si}(10)$ & $133.9(2)$ & $\mathrm{O}(6)-\mathrm{K}(1)-\mathrm{O}(7)$ & $116.40(18)$ \\
\hline N(4)-Hf(2)-Si(10) & $84.9(2)$ & $\mathrm{O}(5)-\mathrm{K}(1)-\mathrm{O}(7)$ & $83.8(2)$ \\
\hline $\operatorname{Si}(7)-H f(2)-S i(10)$ & $70.60(7)$ & $\mathrm{O}(3)-\mathrm{K}(1)-\mathrm{O}(7)$ & $79.92(17)$ \\
\hline $\mathrm{N}(8)-\mathrm{Hf}(3)-\mathrm{N}(7)$ & $109.8(3)$ & $\mathrm{O}(4)-\mathrm{K}(1)-\mathrm{O}(7)$ & $87.23(17)$ \\
\hline $\mathrm{N}(8)-\mathrm{Hf}(3)-\mathrm{N}(9)$ & $98.0(3)$ & $\mathrm{O}(1)-\mathrm{K}(1)-\mathrm{O}(7)$ & $156.24(16)$ \\
\hline N(7)-Hf(3)-N(9) & $97.4(3)$ & $\mathrm{O}(2)-\mathrm{K}(1)-\mathrm{O}(7)$ & $121.39(17)$ \\
\hline N(8)-Hf(3)-Si(16) & $94.98(19)$ & $\mathrm{O}(16)-\mathrm{K}(2)-\mathrm{O}(20)$ & $123.4(2)$ \\
\hline N(7)-Hf(3)-Si(16) & $97.2(2)$ & $\mathrm{O}(16)-\mathrm{K}(2)-\mathrm{O}(17)$ & $58.7(3)$ \\
\hline N(9)-Hf(3)-Si(16) & $155.9(2)$ & $\mathrm{O}(20)-\mathrm{K}(2)-\mathrm{O}(17)$ & $106.8(2)$ \\
\hline $\mathrm{N}(8)-\mathrm{Hf}(3)-\mathrm{Si}(13)$ & $125.34(19)$ & $\mathrm{O}(16)-\mathrm{K}(2)-\mathrm{O}(14)$ & $118.3(3)$ \\
\hline N(7)-Hf(3)-Si(13) & $123.9(2)$ & $\mathrm{O}(20)-\mathrm{K}(2)-\mathrm{O}(14)$ & $93.3(2)$ \\
\hline $\mathrm{N}(9)-\mathrm{Hf}(3)-\mathrm{Si}(13)$ & $85.3(2)$ & $\mathrm{O}(17)-\mathrm{K}(2)-\mathrm{O}(14)$ & $157.4(3)$ \\
\hline $\operatorname{Si}(16)-\mathrm{Hf}(3)-\mathrm{Si}(13)$ & $70.61(7)$ & $\mathrm{O}(16)-\mathrm{K}(2)-\mathrm{O}(15)$ & $59.7(3)$ \\
\hline $\mathrm{N}(10)-\mathrm{Hf}(4)-\mathrm{N}(12)$ & $106.7(4)$ & $\mathrm{O}(20)-\mathrm{K}(2)-\mathrm{O}(15)$ & $125.3(2)$ \\
\hline $\mathrm{N}(10)-\mathrm{Hf}(4)-\mathrm{N}(11)$ & $101.8(3)$ & $\mathrm{O}(17)-\mathrm{K}(2)-\mathrm{O}(15)$ & $114.0(3)$ \\
\hline $\mathrm{N}(12)-\mathrm{Hf}(4)-\mathrm{N}(11)$ & $96.3(4)$ & $\mathrm{O}(14)-\mathrm{K}(2)-\mathrm{O}(15)$ & $58.6(3)$ \\
\hline $\mathrm{N}(10)-\mathrm{Hf}(4)-\mathrm{Si}(22)$ & $106.7(2)$ & $\mathrm{O}(16)-\mathrm{K}(2)-\mathrm{O}(18)$ & $118.3(3)$ \\
\hline $\mathrm{N}(12)-\mathrm{Hf}(4)-\mathrm{Si}(22)$ & $144.1(3)$ & $\mathrm{O}(20)-\mathrm{K}(2)-\mathrm{O}(18)$ & $79.00(19)$ \\
\hline N(11)-Hf(4)-Si(22) & $89.5(2)$ & $\mathrm{O}(17)-\mathrm{K}(2)-\mathrm{O}(18)$ & $59.8(3)$ \\
\hline $\mathrm{N}(10)-\mathrm{Hf}(4)-\mathrm{Si}(19)$ & $105.6(2)$ & $\mathrm{O}(14)-\mathrm{K}(2)-\mathrm{O}(18)$ & $116.1(3)$ \\
\hline $\mathrm{N}(12)-\mathrm{Hf}(4)-\mathrm{Si}(19)$ & $88.5(2)$ & $\mathrm{O}(15)-\mathrm{K}(2)-\mathrm{O}(18)$ & $153.8(2)$ \\
\hline N(11)-Hf(4)-Si(19) & $149.5(2)$ & $\mathrm{O}(16)-\mathrm{K}(2)-\mathrm{O}(13)$ & $151.5(3)$ \\
\hline $\mathrm{Si}(22)-\mathrm{Hf}(4)-\mathrm{Si}(19)$ & $70.12(7)$ & $\mathrm{O}(20)-\mathrm{K}(2)-\mathrm{O}(13)$ & $84.7(2)$ \\
\hline $\mathrm{O}(8)-\mathrm{K}(1)-\mathrm{O}(6)$ & $120.19(19)$ & $\mathrm{O}(17)-\mathrm{K}(2)-\mathrm{O}(13)$ & $112.5(4)$ \\
\hline $\mathrm{O}(8)-\mathrm{K}(1)-\mathrm{O}(5)$ & $138.3(2)$ & $\mathrm{O}(14)-\mathrm{K}(2)-\mathrm{O}(13)$ & $58.2(4)$ \\
\hline $\mathrm{O}(6)-\mathrm{K}(1)-\mathrm{O}(5)$ & $58.45(18)$ & $\mathrm{O}(15)-\mathrm{K}(2)-\mathrm{O}(13)$ & $110.0(3)$ \\
\hline $\mathrm{O}(8)-\mathrm{K}(1)-\mathrm{O}(3)$ & $77.53(18)$ & $\mathrm{O}(18)-\mathrm{K}(2)-\mathrm{O}(13)$ & $57.9(3)$ \\
\hline $\mathrm{O}(6)-\mathrm{K}(1)-\mathrm{O}(3)$ & $160.0(2)$ & $\mathrm{O}(16)-\mathrm{K}(2)-\mathrm{O}(19)$ & $77.0(2)$ \\
\hline $\mathrm{O}(5)-\mathrm{K}(1)-\mathrm{O}(3)$ & $115.8(2)$ & $\mathrm{O}(20)-\mathrm{K}(2)-\mathrm{O}(19)$ & 56.91(16) \\
\hline $\mathrm{O}(8)-\mathrm{K}(1)-\mathrm{O}(4)$ & $128.74(18)$ & $\mathrm{O}(17)-\mathrm{K}(2)-\mathrm{O}(19)$ & $110.9(2)$ \\
\hline $\mathrm{O}(6)-\mathrm{K}(1)-\mathrm{O}(4)$ & 108.67(19) & $\mathrm{O}(14)-\mathrm{K}(2)-\mathrm{O}(19)$ & $88.5(3)$ \\
\hline $\mathrm{O}(5)-\mathrm{K}(1)-\mathrm{O}(4)$ & $59.3(2)$ & $\mathrm{O}(15)-\mathrm{K}(2)-\mathrm{O}(19)$ & 75.01(18) \\
\hline $\mathrm{O}(3)-\mathrm{K}(1)-\mathrm{O}(4)$ & $58.26(19)$ & $\mathrm{O}(18)-\mathrm{K}(2)-\mathrm{O}(19)$ & $131.17(19)$ \\
\hline $\mathrm{O}(8)-\mathrm{K}(1)-\mathrm{O}(1)$ & $102.17(16)$ & $\mathrm{O}(13)-\mathrm{K}(2)-\mathrm{O}(19)$ & $128.5(3)$ \\
\hline $\mathrm{O}(6)-\mathrm{K}(1)-\mathrm{O}(1)$ & $58.37(18)$ & $\mathrm{O}(25)-\mathrm{K}(3)-\mathrm{O}(26)$ & $61.5(2)$ \\
\hline $\mathrm{O}(5)-\mathrm{K}(1)-\mathrm{O}(1)$ & $107.8(2)$ & $\mathrm{O}(25)-\mathrm{K}(3)-\mathrm{O}(28)$ & $179.8(3)$ \\
\hline $\mathrm{O}(3)-\mathrm{K}(1)-\mathrm{O}(1)$ & $111.47(19)$ & $\mathrm{O}(26)-\mathrm{K}(3)-\mathrm{O}(28)$ & $118.7(2)$ \\
\hline $\mathrm{O}(4)-\mathrm{K}(1)-\mathrm{O}(1)$ & $116.53(17)$ & $\mathrm{O}(25)-\mathrm{K}(3)-\mathrm{O}(27)$ & $119.8(2)$ \\
\hline $\mathrm{O}(8)-\mathrm{K}(1)-\mathrm{O}(2)$ & $72.15(17)$ & $\mathrm{O}(26)-\mathrm{K}(3)-\mathrm{O}(27)$ & $60.3(2)$ \\
\hline $\mathrm{O}(6)-\mathrm{K}(1)-\mathrm{O}(2)$ & $114.88(19)$ & $\mathrm{O}(28)-\mathrm{K}(3)-\mathrm{O}(27)$ & $60.4(2)$ \\
\hline $\mathrm{O}(5)-\mathrm{K}(1)-\mathrm{O}(2)$ & $149.4(2)$ & $\mathrm{O}(25)-\mathrm{K}(3)-\mathrm{O}(29)$ & $119.4(2)$ \\
\hline $\mathrm{O}(3)-\mathrm{K}(1)-\mathrm{O}(2)$ & 58.91(19) & $\mathrm{O}(26)-\mathrm{K}(3)-\mathrm{O}(29)$ & $174.8(2)$ \\
\hline $\mathrm{O}(4)-\mathrm{K}(1)-\mathrm{O}(2)$ & 101.64(19) & $\mathrm{O}(28)-\mathrm{K}(3)-\mathrm{O}(29)$ & $60.4(2)$ \\
\hline $\mathrm{O}(1)-\mathrm{K}(1)-\mathrm{O}(2)$ & $56.57(18)$ & $\mathrm{O}(27)-\mathrm{K}(3)-\mathrm{O}(29)$ & $120.0(2)$ \\
\hline
\end{tabular}




\begin{tabular}{|c|c|c|c|}
\hline $\mathrm{O}(25)-\mathrm{K}(3)-\mathrm{O}(30)$ & $60.6(2)$ & $\mathrm{O}(31) \# 2-\mathrm{K}(4)-\mathrm{C}(165) \# 2$ & $80.9(2)$ \\
\hline $\mathrm{O}(26)-\mathrm{K}(3)-\mathrm{O}(30)$ & $119.9(2)$ & $\mathrm{O}(31)-\mathrm{K}(4)-\mathrm{C}(165) \# 2$ & $99.1(2)$ \\
\hline $\mathrm{O}(28)-\mathrm{K}(3)-\mathrm{O}(30)$ & $119.2(2)$ & $\mathrm{O}(33)-\mathrm{K}(4)-\mathrm{C}(165)$ & $101.7(2)$ \\
\hline $\mathrm{O}(27)-\mathrm{K}(3)-\mathrm{O}(30)$ & $179.0(2)$ & $\mathrm{O}(33) \# 2-\mathrm{K}(4)-\mathrm{C}(165)$ & $78.3(2)$ \\
\hline $\mathrm{O}(29)-\mathrm{K}(3)-\mathrm{O}(30)$ & $59.7(2)$ & $\mathrm{O}(32)-\mathrm{K}(4)-\mathrm{C}(165)$ & $80.4(3)$ \\
\hline $\mathrm{O}(25)-\mathrm{K}(3)-\mathrm{C}(149)$ & $96.0(3)$ & $\mathrm{O}(32) \# 2-\mathrm{K}(4)-\mathrm{C}(165)$ & $99.6(3)$ \\
\hline $\mathrm{O}(26)-\mathrm{K}(3)-\mathrm{C}(149)$ & $97.2(2)$ & $\mathrm{O}(31) \# 2-\mathrm{K}(4)-\mathrm{C}(165)$ & $99.1(2)$ \\
\hline $\mathrm{O}(28)-\mathrm{K}(3)-\mathrm{C}(149)$ & $84.0(3)$ & $\mathrm{O}(31)-\mathrm{K}(4)-\mathrm{C}(165)$ & $80.9(2)$ \\
\hline $\mathrm{O}(27)-\mathrm{K}(3)-\mathrm{C}(149)$ & $104.9(3)$ & $C(165) \# 2-K(4)-C(165)$ & $180.00(16)$ \\
\hline $\mathrm{O}(29)-\mathrm{K}(3)-\mathrm{C}(149)$ & $77.7(2)$ & $\mathrm{O}(36)-\mathrm{K}(5)-\mathrm{O}(36) \# 3$ & $180.0(2)$ \\
\hline $\mathrm{O}(30)-\mathrm{K}(3)-\mathrm{C}(149)$ & $74.1(2)$ & $\mathrm{O}(36)-\mathrm{K}(5)-\mathrm{O}(34) \# 3$ & $61.33(19)$ \\
\hline $\mathrm{O}(25)-\mathrm{K}(3)-\mathrm{C}(145)$ & $77.5(3)$ & $\mathrm{O}(36) \# 3-\mathrm{K}(5)-\mathrm{O}(34) \# 3$ & $118.67(19)$ \\
\hline $\mathrm{O}(26)-\mathrm{K}(3)-\mathrm{C}(145)$ & $79.6(2)$ & $\mathrm{O}(36)-\mathrm{K}(5)-\mathrm{O}(34)$ & $118.67(19)$ \\
\hline $\mathrm{O}(28)-\mathrm{K}(3)-\mathrm{C}(145)$ & $102.6(3)$ & $\mathrm{O}(36) \# 3-\mathrm{K}(5)-\mathrm{O}(34)$ & $61.33(19)$ \\
\hline $\mathrm{O}(27)-\mathrm{K}(3)-\mathrm{C}(145)$ & $78.6(2)$ & $\mathrm{O}(34) \# 3-\mathrm{K}(5)-\mathrm{O}(34)$ & $179.997(1)$ \\
\hline $\mathrm{O}(29)-\mathrm{K}(3)-\mathrm{C}(145)$ & $105.6(2)$ & $\mathrm{O}(36)-\mathrm{K}(5)-\mathrm{O}(35) \# 3$ & $119.16(19)$ \\
\hline $\mathrm{O}(30)-\mathrm{K}(3)-\mathrm{C}(145)$ & $102.4(2)$ & $\mathrm{O}(36) \# 3-\mathrm{K}(5)-\mathrm{O}(35) \# 3$ & $60.84(19)$ \\
\hline C(149)-K(3)-C(145) & $173.4(3)$ & $\mathrm{O}(34) \# 3-\mathrm{K}(5)-\mathrm{O}(35) \# 3$ & $59.32(18)$ \\
\hline $\mathrm{O}(25)-\mathrm{K}(3)-\mathrm{C}(146)$ & $101.1(2)$ & $\mathrm{O}(34)-\mathrm{K}(5)-\mathrm{O}(35) \# 3$ & $120.67(18)$ \\
\hline $\mathrm{O}(26)-\mathrm{K}(3)-\mathrm{C}(146)$ & $93.7(2)$ & $\mathrm{O}(36)-\mathrm{K}(5)-\mathrm{O}(35)$ & $60.84(19)$ \\
\hline $\mathrm{O}(28)-\mathrm{K}(3)-\mathrm{C}(146)$ & $79.0(2)$ & $\mathrm{O}(36) \# 3-\mathrm{K}(5)-\mathrm{O}(35)$ & $119.16(19)$ \\
\hline $\mathrm{O}(27)-\mathrm{K}(3)-\mathrm{C}(146)$ & $69.2(2)$ & $\mathrm{O}(34) \# 3-\mathrm{K}(5)-\mathrm{O}(35)$ & $120.68(18)$ \\
\hline $\mathrm{O}(29)-\mathrm{K}(3)-\mathrm{C}(146)$ & $91.1(2)$ & $\mathrm{O}(34)-\mathrm{K}(5)-\mathrm{O}(35)$ & $59.33(18)$ \\
\hline $\mathrm{O}(30)-\mathrm{K}(3)-\mathrm{C}(146)$ & $111.7(2)$ & $\mathrm{O}(35) \# 3-\mathrm{K}(5)-\mathrm{O}(35)$ & $180.00(15)$ \\
\hline $\mathrm{C}(149)-\mathrm{K}(3)-\mathrm{C}(146)$ & $162.7(3)$ & $\mathrm{O}(36)-\mathrm{K}(5)-\mathrm{C}(178)$ & $80.5(2)$ \\
\hline$C(145)-K(3)-C(146)$ & $23.8(3)$ & $\mathrm{O}(36) \# 3-\mathrm{K}(5)-\mathrm{C}(178)$ & $99.5(2)$ \\
\hline $\mathrm{O}(33)-\mathrm{K}(4)-\mathrm{O}(33) \# 2$ & $179.999(2)$ & $\mathrm{O}(34) \# 3-\mathrm{K}(5)-\mathrm{C}(178)$ & $78.4(2)$ \\
\hline $\mathrm{O}(33)-\mathrm{K}(4)-\mathrm{O}(32)$ & $61.1(2)$ & $\mathrm{O}(34)-\mathrm{K}(5)-\mathrm{C}(178)$ & $101.6(2)$ \\
\hline $\mathrm{O}(33) \# 2-\mathrm{K}(4)-\mathrm{O}(32)$ & $118.9(2)$ & $\mathrm{O}(35) \# 3-\mathrm{K}(5)-\mathrm{C}(178)$ & $76.0(2)$ \\
\hline $\mathrm{O}(33)-\mathrm{K}(4)-\mathrm{O}(32) \# 2$ & $118.9(2)$ & $\mathrm{O}(35)-\mathrm{K}(5)-\mathrm{C}(178)$ & $104.0(2)$ \\
\hline $\mathrm{O}(33) \# 2-\mathrm{K}(4)-\mathrm{O}(32) \# 2$ & $61.1(2)$ & $\mathrm{O}(36)-\mathrm{K}(5)-\mathrm{C}(178) \# 3$ & $99.5(2)$ \\
\hline $\mathrm{O}(32)-\mathrm{K}(4)-\mathrm{O}(32) \# 2$ & $180.00(14)$ & $\mathrm{O}(36) \# 3-\mathrm{K}(5)-\mathrm{C}(178) \# 3$ & $80.5(2)$ \\
\hline $\mathrm{O}(33)-\mathrm{K}(4)-\mathrm{O}(31) \# 2$ & $60.8(2)$ & $\mathrm{O}(34) \# 3-\mathrm{K}(5)-\mathrm{C}(178) \# 3$ & $101.6(2)$ \\
\hline $\mathrm{O}(33) \# 2-\mathrm{K}(4)-\mathrm{O}(31) \# 2$ & $119.2(2)$ & $\mathrm{O}(34)-\mathrm{K}(5)-\mathrm{C}(178) \# 3$ & $78.4(2)$ \\
\hline $\mathrm{O}(32)-\mathrm{K}(4)-\mathrm{O}(31) \# 2$ & $120.38(19)$ & $\mathrm{O}(35) \# 3-\mathrm{K}(5)-\mathrm{C}(178) \# 3$ & $104.0(2)$ \\
\hline $\mathrm{O}(32) \# 2-\mathrm{K}(4)-\mathrm{O}(31) \# 2$ & $59.62(19)$ & $\mathrm{O}(35)-\mathrm{K}(5)-\mathrm{C}(178) \# 3$ & $76.0(2)$ \\
\hline $\mathrm{O}(33)-\mathrm{K}(4)-\mathrm{O}(31)$ & $119.2(2)$ & $\mathrm{C}(178)-\mathrm{K}(5)-\mathrm{C}(178) \# 3$ & $179.999(1)$ \\
\hline $\mathrm{O}(33) \# 2-\mathrm{K}(4)-\mathrm{O}(31)$ & $60.8(2)$ & $\mathrm{O}(36)-\mathrm{K}(5)-\mathrm{C}(177) \# 3$ & $107.7(2)$ \\
\hline $\mathrm{O}(32)-\mathrm{K}(4)-\mathrm{O}(31)$ & $59.62(19)$ & $\mathrm{O}(36) \# 3-\mathrm{K}(5)-\mathrm{C}(177) \# 3$ & $72.3(2)$ \\
\hline $\mathrm{O}(32) \# 2-\mathrm{K}(4)-\mathrm{O}(31)$ & $120.38(19)$ & $\mathrm{O}(34) \# 3-\mathrm{K}(5)-\mathrm{C}(177) \# 3$ & $86.6(2)$ \\
\hline $\mathrm{O}(31) \# 2-\mathrm{K}(4)-\mathrm{O}(31)$ & $179.998(1)$ & $\mathrm{O}(34)-\mathrm{K}(5)-\mathrm{C}(177) \# 3$ & $93.4(2)$ \\
\hline $\mathrm{O}(33)-\mathrm{K}(4)-\mathrm{C}(165) \# 2$ & $78.3(2)$ & $\mathrm{O}(35) \# 3-\mathrm{K}(5)-\mathrm{C}(177) \# 3$ & $81.1(2)$ \\
\hline $\mathrm{O}(33) \# 2-\mathrm{K}(4)-\mathrm{C}(165) \# 2$ & $101.7(2)$ & $\mathrm{O}(35)-\mathrm{K}(5)-\mathrm{C}(177) \# 3$ & $98.9(2)$ \\
\hline $\mathrm{O}(32)-\mathrm{K}(4)-\mathrm{C}(165) \# 2$ & $99.6(3)$ & $\mathrm{C}(178)-\mathrm{K}(5)-\mathrm{C}(177) \# 3$ & $156.8(2)$ \\
\hline $\mathrm{O}(32) \# 2-\mathrm{K}(4)-\mathrm{C}(165) \# 2$ & $80.4(3)$ & $\mathrm{C}(178) \# 3-\mathrm{K}(5)-\mathrm{C}(177) \# 3$ & $23.2(2)$ \\
\hline
\end{tabular}




\begin{tabular}{|c|c|c|c|}
\hline $\mathrm{O}(36)-\mathrm{K}(5)-\mathrm{C}(177)$ & $72.3(2)$ & $\mathrm{C}(92)-\mathrm{O}(1)-\mathrm{C}(81)$ & $112.0(7)$ \\
\hline $\mathrm{O}(36) \# 3-\mathrm{K}(5)-\mathrm{C}(177)$ & $107.7(2)$ & $\mathrm{C}(92)-\mathrm{O}(1)-\mathrm{K}(1)$ & $110.0(5)$ \\
\hline $\mathrm{O}(34) \# 3-\mathrm{K}(5)-\mathrm{C}(177)$ & $93.4(2)$ & $\mathrm{C}(81)-\mathrm{O}(1)-\mathrm{K}(1)$ & $114.6(5)$ \\
\hline $\mathrm{O}(34)-\mathrm{K}(5)-\mathrm{C}(177)$ & $86.6(2)$ & $\mathrm{C}(82)-\mathrm{O}(2)-\mathrm{C}(83)$ & $112.8(7)$ \\
\hline $\mathrm{O}(35) \# 3-\mathrm{K}(5)-\mathrm{C}(177)$ & $98.9(2)$ & $\mathrm{C}(82)-\mathrm{O}(2)-\mathrm{K}(1)$ & $119.5(5)$ \\
\hline $\mathrm{O}(35)-\mathrm{K}(5)-\mathrm{C}(177)$ & $81.1(2)$ & $\mathrm{C}(83)-\mathrm{O}(2)-\mathrm{K}(1)$ & $115.8(5)$ \\
\hline $\mathrm{C}(178)-\mathrm{K}(5)-\mathrm{C}(177)$ & $23.2(2)$ & $\mathrm{C}(85)-\mathrm{O}(3)-\mathrm{C}(84)$ & $115.9(8)$ \\
\hline $\mathrm{C}(178) \# 3-\mathrm{K}(5)-\mathrm{C}(177)$ & $156.8(2)$ & $\mathrm{C}(85)-\mathrm{O}(3)-\mathrm{K}(1)$ & $114.2(6)$ \\
\hline $\mathrm{C}(177) \# 3-\mathrm{K}(5)-\mathrm{C}(177)$ & $180.000(2)$ & $\mathrm{C}(84)-\mathrm{O}(3)-\mathrm{K}(1)$ & $112.8(5)$ \\
\hline $\mathrm{C}(2)-\mathrm{N}(1)-\mathrm{C}(1)$ & $106.5(8)$ & $\mathrm{C}(86)-\mathrm{O}(4)-\mathrm{C}(87)$ & $109.1(7)$ \\
\hline $\mathrm{C}(2)-\mathrm{N}(1)-\mathrm{Hf}(1)$ & $141.0(6)$ & $\mathrm{C}(86)-\mathrm{O}(4)-\mathrm{K}(1)$ & $116.9(5)$ \\
\hline $\mathrm{C}(1)-\mathrm{N}(1)-\mathrm{Hf}(1)$ & $112.5(6)$ & $\mathrm{C}(87)-\mathrm{O}(4)-\mathrm{K}(1)$ & $113.4(5)$ \\
\hline $\mathrm{C}(5)-\mathrm{N}(2)-\mathrm{C}(6)$ & $111.2(8)$ & $\mathrm{C}(89)-\mathrm{O}(5)-\mathrm{C}(88)$ & $114.3(11)$ \\
\hline $\mathrm{C}(5)-\mathrm{N}(2)-\mathrm{Hf}(1)$ & $129.0(7)$ & $\mathrm{C}(89)-\mathrm{O}(5)-\mathrm{K}(1)$ & $115.3(6)$ \\
\hline $\mathrm{C}(6)-\mathrm{N}(2)-\mathrm{Hf}(1)$ & $119.7(6)$ & $\mathrm{C}(88)-\mathrm{O}(5)-\mathrm{K}(1)$ & $115.6(6)$ \\
\hline $\mathrm{C}(4)-\mathrm{N}(3)-\mathrm{C}(3)$ & $113.0(8)$ & $\mathrm{C}(91)-\mathrm{O}(6)-\mathrm{C}(90)$ & $112.6(10)$ \\
\hline $\mathrm{C}(4)-\mathrm{N}(3)-\mathrm{Hf}(1)$ & $133.0(6)$ & $\mathrm{C}(91)-\mathrm{O}(6)-\mathrm{K}(1)$ & $118.7(6)$ \\
\hline $\mathrm{C}(3)-\mathrm{N}(3)-\mathrm{Hf}(1)$ & $113.3(6)$ & $\mathrm{C}(90)-\mathrm{O}(6)-\mathrm{K}(1)$ & $117.5(6)$ \\
\hline $\mathrm{C}(21)-\mathrm{N}(4)-\mathrm{C}(22)$ & $106.9(7)$ & $\mathrm{C}(104)-\mathrm{O}(7)-\mathrm{C}(93)$ & $112.8(7)$ \\
\hline $\mathrm{C}(21)-\mathrm{N}(4)-\mathrm{Hf}(2)$ & $140.1(6)$ & $\mathrm{C}(104)-\mathrm{O}(7)-\mathrm{K}(1)$ & $115.4(5)$ \\
\hline $\mathrm{C}(22)-\mathrm{N}(4)-\mathrm{Hf}(2)$ & $112.9(5)$ & $\mathrm{C}(93)-\mathrm{O}(7)-\mathrm{K}(1)$ & $102.4(5)$ \\
\hline $\mathrm{C}(23)-\mathrm{N}(5)-\mathrm{C}(24)$ & $114.0(7)$ & $\mathrm{C}(94)-\mathrm{O}(8)-\mathrm{C}(95)$ & $114.0(6)$ \\
\hline $\mathrm{C}(23)-\mathrm{N}(5)-\mathrm{Hf}(2)$ & $111.4(5)$ & $\mathrm{C}(94)-\mathrm{O}(8)-\mathrm{K}(1)$ & $125.8(4)$ \\
\hline $\mathrm{C}(24)-\mathrm{N}(5)-\mathrm{Hf}(2)$ & $133.9(6)$ & $\mathrm{C}(95)-\mathrm{O}(8)-\mathrm{K}(1)$ & $116.3(5)$ \\
\hline$C(25)-N(6)-C(26)$ & $112.7(7)$ & $\mathrm{C}(97)-\mathrm{O}(9)-\mathrm{C}(96)$ & $109.6(6)$ \\
\hline $\mathrm{C}(25)-\mathrm{N}(6)-\mathrm{Hf}(2)$ & $127.0(7)$ & $\mathrm{C}(98)-\mathrm{O}(10)-\mathrm{C}(99)$ & $114.9(7)$ \\
\hline $\mathrm{C}(26)-\mathrm{N}(6)-\mathrm{Hf}(2)$ & $120.2(5)$ & $\mathrm{C}(101)-\mathrm{O}(11)-\mathrm{C}(100)$ & $113.7(7)$ \\
\hline $\mathrm{C}(41)-\mathrm{N}(7)-\mathrm{C}(42)$ & $110.9(7)$ & $\mathrm{C}(103)-\mathrm{O}(12)-\mathrm{C}(102)$ & $112.5(7)$ \\
\hline $\mathrm{C}(41)-\mathrm{N}(7)-\mathrm{Hf}(3)$ & $117.6(6)$ & $\mathrm{C}(105)-\mathrm{O}(13)-\mathrm{C}(116)$ & $118.3(12)$ \\
\hline C(42)-N(7)-Hf(3) & $131.5(6)$ & $\mathrm{C}(105)-\mathrm{O}(13)-\mathrm{K}(2)$ & $109.3(10)$ \\
\hline $\mathrm{C}(44)-\mathrm{N}(8)-\mathrm{C}(43)$ & $109.1(7)$ & $\mathrm{C}(116)-\mathrm{O}(13)-\mathrm{K}(2)$ & $112.7(8)$ \\
\hline $\mathrm{C}(44)-\mathrm{N}(8)-\mathrm{Hf}(3)$ & $128.8(6)$ & $\mathrm{C}(107)-\mathrm{O}(14)-\mathrm{C}(106)$ & $106.0(12)$ \\
\hline $\mathrm{C}(43)-\mathrm{N}(8)-\mathrm{Hf}(3)$ & $121.8(5)$ & $\mathrm{C}(107)-\mathrm{O}(14)-\mathrm{K}(2)$ & $121.4(9)$ \\
\hline$C(46)-N(9)-C(45)$ & $108.3(7)$ & $\mathrm{C}(106)-\mathrm{O}(14)-\mathrm{K}(2)$ & $120.7(11)$ \\
\hline $\mathrm{C}(46)-\mathrm{N}(9)-\mathrm{Hf}(3)$ & $140.8(6)$ & $\mathrm{C}(109)-\mathrm{O}(15)-\mathrm{C}(108)$ & $113.2(11)$ \\
\hline $\mathrm{C}(45)-\mathrm{N}(9)-\mathrm{Hf}(3)$ & $110.8(6)$ & $\mathrm{C}(109)-\mathrm{O}(15)-\mathrm{K}(2)$ & $111.0(8)$ \\
\hline $\mathrm{C}(61)-\mathrm{N}(10)-\mathrm{C}(62)$ & $112.5(7)$ & $\mathrm{C}(108)-\mathrm{O}(15)-\mathrm{K}(2)$ & $109.3(7)$ \\
\hline $\mathrm{C}(61)-\mathrm{N}(10)-\mathrm{Hf}(4)$ & $122.1(6)$ & $\mathrm{C}(110)-\mathrm{O}(16)-\mathrm{C}(111)$ & $114.2(10)$ \\
\hline $\mathrm{C}(62)-\mathrm{N}(10)-\mathrm{Hf}(4)$ & $125.2(6)$ & $\mathrm{C}(110)-\mathrm{O}(16)-\mathrm{K}(2)$ & $116.7(8)$ \\
\hline $\mathrm{C}(64)-\mathrm{N}(11)-\mathrm{C}(63)$ & $107.2(8)$ & $\mathrm{C}(111)-\mathrm{O}(16)-\mathrm{K}(2)$ & $118.1(8)$ \\
\hline $\mathrm{C}(64)-\mathrm{N}(11)-\mathrm{Hf}(4)$ & $128.7(6)$ & $\mathrm{C}(113)-\mathrm{O}(17)-\mathrm{C}(112)$ & $112.0(12)$ \\
\hline $\mathrm{C}(63)-\mathrm{N}(11)-\mathrm{Hf}(4)$ & $123.5(8)$ & $\mathrm{C}(113)-\mathrm{O}(17)-\mathrm{K}(2)$ & $109.6(8)$ \\
\hline $\mathrm{C}(65)-\mathrm{N}(12)-\mathrm{C}(66)$ & $108.0(11)$ & $\mathrm{C}(112)-\mathrm{O}(17)-\mathrm{K}(2)$ & $111.8(8)$ \\
\hline $\mathrm{C}(65)-\mathrm{N}(12)-\mathrm{Hf}(4)$ & $132.4(10)$ & $\mathrm{C}(115)-\mathrm{O}(18)-\mathrm{C}(114)$ & $113.4(12)$ \\
\hline $\mathrm{C}(66)-\mathrm{N}(12)-\mathrm{Hf}(4)$ & $119.6(8)$ & $\mathrm{C}(115)-\mathrm{O}(18)-\mathrm{K}(2)$ & $119.0(9)$ \\
\hline
\end{tabular}




$\begin{array}{lr}\mathrm{C}(114)-\mathrm{O}(18)-\mathrm{K}(2) & 112.7(8) \\ \mathrm{C}(117)-\mathrm{O}(19)-\mathrm{C}(128) & 112.3(7) \\ \mathrm{C}(117)-\mathrm{O}(19)-\mathrm{K}(2) & 96.7(5) \\ \mathrm{C}(128)-\mathrm{O}(19)-\mathrm{K}(2) & 126.0(6) \\ \mathrm{C}(118)-\mathrm{O}(20)-\mathrm{C}(119) & 112.6(7) \\ \mathrm{C}(118)-\mathrm{O}(20)-\mathrm{K}(2) & 123.6(5) \\ \mathrm{C}(119)-\mathrm{O}(20)-\mathrm{K}(2) & 123.6(5) \\ \mathrm{C}(120)-\mathrm{O}(21)-\mathrm{C}(121) & 112.1(7) \\ \mathrm{C}(122)-\mathrm{O}(22)-\mathrm{C}(123) & 110.8(8) \\ \mathrm{C}(124)-\mathrm{O}(23)-\mathrm{C}(125) & 104.4(12) \\ \mathrm{C}(127)-\mathrm{O}(24)-\mathrm{C}(126) & 114.3(10) \\ \mathrm{C}(129)-\mathrm{O}(25)-\mathrm{C}(140) & 111.8(8) \\ \mathrm{C}(129)-\mathrm{O}(25)-\mathrm{K}(3) & 115.6(6) \\ \mathrm{C}(140)-\mathrm{O}(25)-\mathrm{K}(3) & 116.6(6) \\ \mathrm{C}(131)-\mathrm{O}(26)-\mathrm{C}(130) & 112.4(8) \\ \mathrm{C}(131)-\mathrm{O}(26)-\mathrm{K}(3) & 117.3(6) \\ \mathrm{C}(130)-\mathrm{O}(26)-\mathrm{K}(3) & 114.1(6) \\ \mathrm{C}(133)-\mathrm{O}(27)-\mathrm{C}(132) & 115.0(8) \\ \mathrm{C}(133)-\mathrm{O}(27)-\mathrm{K}(3) & 116.3(6) \\ \mathrm{C}(132)-\mathrm{O}(27)-\mathrm{K}(3) & 113.5(6) \\ \mathrm{C}(135)-\mathrm{O}(28)-\mathrm{C}(134) & 111.5(8) \\ \mathrm{C}(135)-\mathrm{O}(28)-\mathrm{K}(3) & 114.7(6) \\ \mathrm{C}(134)-\mathrm{O}(28)-\mathrm{K}(3) & 114.2(5) \\ \mathrm{C}(137)-\mathrm{O}(29)-\mathrm{C}(136) & 114.2(8) \\ \mathrm{C}(137)-\mathrm{O}(29)-\mathrm{K}(3) & 116.7(6) \\ \mathrm{C}(136)-\mathrm{O}(29)-\mathrm{K}(3) & 112.5(6) \\ \mathrm{C}(139)-\mathrm{O}(30)-\mathrm{C}(138) & 112.3(8) \\ \mathrm{C}(139)-\mathrm{O}(30)-\mathrm{K}(3) & 113.8(6) \\ \mathrm{C}(138)-\mathrm{O}(30)-\mathrm{K}(3) & 114.3(5) \\ \mathrm{C}(156)-\mathrm{O}(31)-\mathrm{C}(155) & 112.1(8) \\ \mathrm{C}(156)-\mathrm{O}(31)-\mathrm{K}(4) & 115.6(6) \\ \mathrm{C}(155)-\mathrm{O}(31)-\mathrm{K}(4) & 115.7(6) \\ \mathrm{C}(158)-\mathrm{O}(32)-\mathrm{C}(157) & 113.8(8) \\ \mathrm{C}(158)-\mathrm{O}(32)-\mathrm{K}(4) & 112.8(6) \\ \mathrm{C}(157)-\mathrm{O}(32)-\mathrm{K}(4) & 115.0(5) \\ \mathrm{C}(160)-\mathrm{O}(33)-\mathrm{C}(159) & 110.3(8) \\ \mathrm{C}(160)-\mathrm{O}(33)-\mathrm{K}(4) & 115.4(6) \\ \mathrm{C}(159)-\mathrm{O}(33)-\mathrm{K}(4) & 113.3(5) \\ \mathrm{C}(168) \# 3-\mathrm{O}(34)-\mathrm{C}(173) & 112.0(7) \\ \mathrm{C}(168) \# 3-\mathrm{O}(34)-\mathrm{K}(5) & 113.3(5) \\ \mathrm{C}(173)-\mathrm{O}(34)-\mathrm{K}(5) & 117.1(5) \\ \mathrm{C}(171)-\mathrm{O}(35)-\mathrm{C}(172) & 111.7(7) \\ & 113.0(5) \\ \mathrm{C}(5) & 115.8(5) \\ \mathrm{C}(17)-\mathrm{O}(170) & 111.7(7) \\ & \end{array}$

\begin{tabular}{|c|c|}
\hline $\mathrm{C}(169)-\mathrm{O}(36)-\mathrm{K}(5)$ & $114.7(5)$ \\
\hline $\mathrm{C}(170)-\mathrm{O}(36)-\mathrm{K}(5)$ & $115.8(5)$ \\
\hline$C(7)-\operatorname{Si}(1)-\operatorname{Si}(2)$ & $102.0(2)$ \\
\hline $\mathrm{C}(7)-\mathrm{Si}(1)-\mathrm{Si}(3)$ & $101.8(3)$ \\
\hline $\operatorname{Si}(2)-\operatorname{Si}(1)-\operatorname{Si}(3)$ & $105.39(12)$ \\
\hline C(7)-Si(1)-Hf(1) & $112.9(2)$ \\
\hline $\mathrm{Si}(2)-\mathrm{Si}(1)-\mathrm{Hf}(1)$ & $117.60(11)$ \\
\hline $\operatorname{Si}(3)-\operatorname{Si}(1)-H f(1)$ & $115.22(9)$ \\
\hline$C(9)-\operatorname{Si}(2)-C(10)$ & $106.5(5)$ \\
\hline C(9)-Si(2)-C(11) & $105.4(5)$ \\
\hline$C(10)-S i(2)-C(11)$ & $106.5(5)$ \\
\hline $\mathrm{C}(9)-\mathrm{Si}(2)-\mathrm{Si}(1)$ & $108.0(3)$ \\
\hline $\mathrm{C}(10)-\mathrm{Si}(2)-\mathrm{Si}(1)$ & $114.6(3)$ \\
\hline $\mathrm{C}(11)-\mathrm{Si}(2)-\mathrm{Si}(1)$ & $115.1(4)$ \\
\hline $\mathrm{C}(14)-\mathrm{Si}(3)-\mathrm{C}(13)$ & $106.2(4)$ \\
\hline $\mathrm{C}(14)-\mathrm{Si}(3)-\mathrm{C}(12)$ & $105.2(5)$ \\
\hline $\mathrm{C}(13)-\mathrm{Si}(3)-\mathrm{C}(12)$ & $107.5(5)$ \\
\hline $\mathrm{C}(14)-\mathrm{Si}(3)-\mathrm{Si}(1)$ & $111.7(4)$ \\
\hline C(13)-Si(3)-Si(1) & $112.5(3)$ \\
\hline $\mathrm{C}(12)-\mathrm{Si}(3)-\mathrm{Si}(1)$ & $113.2(4)$ \\
\hline $\mathrm{C}(8)-\mathrm{Si}(4)-\mathrm{Si}(5)$ & $100.0(3)$ \\
\hline C(8)-Si(4)-Si(6) & $101.2(3)$ \\
\hline $\operatorname{Si}(5)-\operatorname{Si}(4)-\operatorname{Si}(6)$ & $104.67(11)$ \\
\hline $\mathrm{C}(8)-\mathrm{Si}(4)-\mathrm{Hf}(1)$ & $112.8(2)$ \\
\hline $\mathrm{Si}(5)-\mathrm{Si}(4)-\mathrm{Hf}(1)$ & $116.75(9)$ \\
\hline $\mathrm{Si}(6)-\mathrm{Si}(4)-\mathrm{Hf}(1)$ & $118.69(10)$ \\
\hline$C(15)-\operatorname{Si}(5)-C(16)$ & $106.2(4)$ \\
\hline $\mathrm{C}(15)-\mathrm{Si}(5)-\mathrm{C}(17)$ & $105.2(4)$ \\
\hline$C(16)-\mathrm{Si}(5)-\mathrm{C}(17)$ & $106.3(4)$ \\
\hline $\mathrm{C}(15)-\mathrm{Si}(5)-\mathrm{Si}(4)$ & $110.1(3)$ \\
\hline$C(16)-\operatorname{Si}(5)-\operatorname{Si}(4)$ & $115.4(3)$ \\
\hline$C(17)-\operatorname{Si}(5)-\operatorname{Si}(4)$ & $112.9(3)$ \\
\hline$C(20)-\operatorname{Si}(6)-C(19)$ & $105.3(5)$ \\
\hline$C(20)-\operatorname{Si}(6)-C(18)$ & $104.9(5)$ \\
\hline$C(19)-\operatorname{Si}(6)-C(18)$ & $106.6(5)$ \\
\hline $\mathrm{C}(20)-\mathrm{Si}(6)-\mathrm{Si}(4)$ & $111.4(3)$ \\
\hline$C(19)-\operatorname{Si}(6)-\operatorname{Si}(4)$ & $110.7(4)$ \\
\hline$C(18)-\operatorname{Si}(6)-\operatorname{Si}(4)$ & $117.1(3)$ \\
\hline C(27)-Si(7)-Si(8) & $104.6(3)$ \\
\hline $\mathrm{C}(27)-\mathrm{Si}(7)-\mathrm{Si}(9)$ & $100.0(3)$ \\
\hline $\operatorname{Si}(8)-\operatorname{Si}(7)-\operatorname{Si}(9)$ & $103.29(11)$ \\
\hline $\mathrm{C}(27)-\mathrm{Si}(7)-\mathrm{Hf}(2)$ & $114.1(2)$ \\
\hline $\operatorname{Si}(8)-\operatorname{Si}(7)-H f(2)$ & $113.19(9)$ \\
\hline $\operatorname{Si}(9)-\operatorname{Si}(7)-H f(2)$ & $119.74(10)$ \\
\hline$C(30)-\operatorname{Si}(8)-C(29)$ & $108.5(4)$ \\
\hline
\end{tabular}




\begin{tabular}{|c|c|c|c|}
\hline$C(30)-\operatorname{Si}(8)-C(31)$ & $106.0(4)$ & $C(52)-\operatorname{Si}(15)-\operatorname{Si}(13)$ & $116.3(3)$ \\
\hline$C(29)-\operatorname{Si}(8)-C(31)$ & $104.6(4)$ & C(54)-Si(15)-Si(13) & $112.4(3)$ \\
\hline C(30)-Si(8)-Si(7) & $111.3(3)$ & $\mathrm{C}(48)-\mathrm{Si}(16)-\mathrm{Si}(17)$ & $103.0(2)$ \\
\hline C(29)-Si(8)-Si(7) & $110.2(3)$ & C(48)-Si(16)-Si(18) & $101.2(3)$ \\
\hline C(31)-Si(8)-Si(7) & $115.8(3)$ & $\operatorname{Si}(17)-\operatorname{Si}(16)-\operatorname{Si}(18)$ & $105.72(11)$ \\
\hline $\mathrm{C}(32)-\mathrm{Si}(9)-\mathrm{C}(34)$ & $108.7(5)$ & $\mathrm{C}(48)-\mathrm{Si}(16)-\mathrm{Hf}(3)$ & $111.9(2)$ \\
\hline C(32)-Si(9)-C(33) & $105.1(5)$ & $\operatorname{Si}(17)-\operatorname{Si}(16)-H f(3)$ & $118.70(10)$ \\
\hline C(34)-Si(9)-C(33) & $105.7(4)$ & $\operatorname{Si}(18)-\operatorname{Si}(16)-H f(3)$ & $114.33(9)$ \\
\hline C(32)-Si(9)-Si(7) & $116.2(3)$ & $C(57)-\operatorname{Si}(17)-C(55)$ & $107.3(4)$ \\
\hline C(34)-Si(9)-Si(7) & $109.1(3)$ & $C(57)-\operatorname{Si}(17)-C(56)$ & $104.4(4)$ \\
\hline C(33)-Si(9)-Si(7) & $111.6(3)$ & $C(55)-\operatorname{Si}(17)-C(56)$ & $108.5(5)$ \\
\hline C(28)-Si(10)-Si(11) & $97.8(2)$ & $\mathrm{C}(57)-\mathrm{Si}(17)-\mathrm{Si}(16)$ & $115.0(3)$ \\
\hline C(28)-Si(10)-Si(12) & $103.2(3)$ & $\mathrm{C}(55)-\mathrm{Si}(17)-\mathrm{Si}(16)$ & $107.7(3)$ \\
\hline $\operatorname{Si}(11)-\operatorname{Si}(10)-\operatorname{Si}(12)$ & $104.89(11)$ & $\mathrm{C}(56)-\mathrm{Si}(17)-\mathrm{Si}(16)$ & $113.6(3)$ \\
\hline $\mathrm{C}(28)-\mathrm{Si}(10)-\mathrm{Hf}(2)$ & $112.1(3)$ & $\mathrm{C}(60)-\mathrm{Si}(18)-\mathrm{C}(58)$ & $108.7(4)$ \\
\hline $\operatorname{Si}(11)-\operatorname{Si}(10)-H f(2)$ & $124.73(10)$ & $\mathrm{C}(60)-\mathrm{Si}(18)-\mathrm{C}(59)$ & $104.7(4)$ \\
\hline $\operatorname{Si}(12)-\operatorname{Si}(10)-H f(2)$ & $111.57(10)$ & $\mathrm{C}(58)-\mathrm{Si}(18)-\mathrm{C}(59)$ & $105.6(4)$ \\
\hline $\mathrm{C}(37)-\mathrm{Si}(11)-\mathrm{C}(35)$ & $105.0(4)$ & $\mathrm{C}(60)-\mathrm{Si}(18)-\mathrm{Si}(16)$ & $113.5(3)$ \\
\hline$C(37)-\operatorname{Si}(11)-C(36)$ & $106.6(5)$ & C(58)-Si(18)-Si(16) & $111.7(3)$ \\
\hline$C(35)-S i(11)-C(36)$ & $104.7(5)$ & C(59)-Si(18)-Si(16) & $112.1(3)$ \\
\hline C(37)-Si(11)-Si(10) & $111.7(3)$ & $\mathrm{C}(68)-\mathrm{Si}(19)-\mathrm{Si}(21)$ & $103.1(3)$ \\
\hline $\mathrm{C}(35)-\mathrm{Si}(11)-\mathrm{Si}(10)$ & $108.7(3)$ & C(68)-Si(19)-Si(20) & $99.8(3)$ \\
\hline C(36)-Si(11)-Si(10) & $119.1(4)$ & $\operatorname{Si}(21)-\operatorname{Si}(19)-\operatorname{Si}(20)$ & $102.23(12)$ \\
\hline $\mathrm{C}(40)-\mathrm{Si}(12)-\mathrm{C}(39)$ & $108.6(6)$ & $\mathrm{C}(68)-\mathrm{Si}(19)-\mathrm{Hf}(4)$ & $112.1(3)$ \\
\hline $\mathrm{C}(40)-\mathrm{Si}(12)-\mathrm{C}(38)$ & $105.3(5)$ & $\mathrm{Si}(21)-\mathrm{Si}(19)-\mathrm{Hf}(4)$ & $113.86(11)$ \\
\hline C(39)-Si(12)-C(38) & $106.8(6)$ & $\operatorname{Si}(20)-\operatorname{Si}(19)-H f(4)$ & $123.16(11)$ \\
\hline C(40)-Si(12)-Si(10) & $111.8(4)$ & $C(70)-\operatorname{Si}(20)-C(71)$ & $106.6(5)$ \\
\hline C(39)-Si(12)-Si(10) & $109.8(4)$ & $C(70)-\operatorname{Si}(20)-C(69)$ & $106.9(5)$ \\
\hline C(38)-Si(12)-Si(10) & $114.3(4)$ & $C(71)-\operatorname{Si}(20)-C(69)$ & $104.5(4)$ \\
\hline C(47)-Si(13)-Si(14) & 99.1(3) & $\mathrm{C}(70)-\mathrm{Si}(20)-\mathrm{Si}(19)$ & $114.4(3)$ \\
\hline $\mathrm{C}(47)-\mathrm{Si}(13)-\mathrm{Si}(15)$ & $101.9(2)$ & $\mathrm{C}(71)-\mathrm{Si}(20)-\mathrm{Si}(19)$ & $115.6(3)$ \\
\hline $\operatorname{Si}(14)-\operatorname{Si}(13)-\operatorname{Si}(15)$ & $106.00(10)$ & C(69)-Si(20)-Si(19) & $108.0(3)$ \\
\hline $\mathrm{C}(47)-\mathrm{Si}(13)-\mathrm{Hf}(3)$ & $113.2(2)$ & $\mathrm{C}(74)-\mathrm{Si}(21)-\mathrm{C}(73)$ & $102.6(5)$ \\
\hline $\mathrm{Si}(14)-\mathrm{Si}(13)-\mathrm{Hf}(3)$ & $117.95(10)$ & $\mathrm{C}(74)-\mathrm{Si}(21)-\mathrm{C}(72)$ & $107.1(5)$ \\
\hline $\operatorname{Si}(15)-\operatorname{Si}(13)-H f(3)$ & $116.19(9)$ & $C(73)-\operatorname{Si}(21)-C(72)$ & $109.4(5)$ \\
\hline$C(50)-\operatorname{Si}(14)-C(49)$ & $104.8(4)$ & $\mathrm{C}(74)-\mathrm{Si}(21)-\mathrm{Si}(19)$ & $112.3(4)$ \\
\hline$C(50)-\operatorname{Si}(14)-C(51)$ & $105.3(4)$ & C(73)-Si(21)-Si(19) & $113.1(4)$ \\
\hline C(49)-Si(14)-C(51) & $105.7(4)$ & C(72)-Si(21)-Si(19) & $111.8(3)$ \\
\hline $\mathrm{C}(50)-\mathrm{Si}(14)-\mathrm{Si}(13)$ & $116.2(3)$ & $\mathrm{C}(67)-\operatorname{Si}(22)-\operatorname{Si}(24)$ & $99.6(3)$ \\
\hline $\mathrm{C}(49)-\mathrm{Si}(14)-\mathrm{Si}(13)$ & $109.8(3)$ & $\mathrm{C}(67)-\mathrm{Si}(22)-\mathrm{Si}(23)$ & $102.7(3)$ \\
\hline $\mathrm{C}(51)-\mathrm{Si}(14)-\mathrm{Si}(13)$ & $114.1(3)$ & $\operatorname{Si}(24)-\operatorname{Si}(22)-\operatorname{Si}(23)$ & $104.39(11)$ \\
\hline $\mathrm{C}(53)-\mathrm{Si}(15)-\mathrm{C}(52)$ & $106.4(4)$ & $\mathrm{C}(67)-\mathrm{Si}(22)-\mathrm{Hf}(4)$ & $113.6(3)$ \\
\hline$C(53)-\operatorname{Si}(15)-C(54)$ & $106.5(4)$ & $\operatorname{Si}(24)-\operatorname{Si}(22)-H f(4)$ & $122.33(12)$ \\
\hline $\mathrm{C}(52)-\mathrm{Si}(15)-\mathrm{C}(54)$ & $104.4(5)$ & $\operatorname{Si}(23)-\operatorname{Si}(22)-H f(4)$ & $111.85(10)$ \\
\hline C(53)-Si(15)-Si(13) & $110.1(3)$ & $\mathrm{C}(75)-\mathrm{Si}(23)-\mathrm{C}(76)$ & $105.8(5)$ \\
\hline
\end{tabular}




$\begin{array}{llll}\mathrm{C}(75)-\mathrm{Si}(23)-\mathrm{C}(77) & 104.8(5) & \mathrm{C}(80)-\mathrm{Si}(24)-\mathrm{C}(79) & 105.0(6) \\ \mathrm{C}(76)-\mathrm{Si}(23)-\mathrm{C}(77) & 106.7(5) & \mathrm{C}(78)-\mathrm{Si}(24)-\mathrm{C}(79) & 105.1(6) \\ \mathrm{C}(75)-\mathrm{Si}(23)-\mathrm{Si}(22) & 111.3(4) & \mathrm{C}(80)-\mathrm{Si}(24)-\mathrm{Si}(22) & 111.1(4) \\ \mathrm{C}(76)-\mathrm{Si}(23)-\mathrm{Si}(22) & 113.2(3) & \mathrm{C}(78)-\mathrm{Si}(24)-\mathrm{Si}(22) & 117.5(3) \\ \mathrm{C}(77)-\mathrm{Si}(23)-\mathrm{Si}(22) & 114.3(3) & \mathrm{C}(79)-\mathrm{Si}(24)-\mathrm{Si}(22) & 111.1(4) \\ \mathrm{C}(80)-\mathrm{Si}(24)-\mathrm{C}(78) & 106.0(6) & & \end{array}$

Symmetry transformations used to generate equivalent atoms:

$\# 1-\mathrm{x}+1,-\mathrm{y}+1,-\mathrm{z}+2 \quad \# 2-\mathrm{x}+1,-\mathrm{y}+1,-\mathrm{z}+1 \quad \# 3-\mathrm{x}+1,-\mathrm{y},-\mathrm{z}+1$ 
Table S19. Anisotropic displacement parameters $\left(\AA^{2} \times 10^{3}\right)$ for 4 . The anisotropic displacement factor exponent takes the form: $-2 \pi^{2}\left(h^{2} a^{* 2} U^{11}+\ldots+2 h k a * b * U^{12}\right)$

\begin{tabular}{lcccccc}
\hline & $\mathrm{U}^{11}$ & $\mathrm{U}^{22}$ & $\mathrm{U}^{33}$ & $\mathrm{U}^{23}$ & $\mathrm{U}^{13}$ & $\mathrm{U}^{12}$ \\
\hline $\mathrm{C}(1)$ & 121 & 117 & 32 & 16 & 20 & 72 \\
$\mathrm{C}(2)$ & 61 & 79 & 57 & 19 & 30 & 45 \\
$\mathrm{C}(3)$ & 88 & 44 & 85 & 14 & -27 & -7 \\
$\mathrm{C}(4)$ & 72 & 73 & 86 & 33 & -34 & 12 \\
$\mathrm{C}(5)$ & 68 & 82 & 87 & 34 & 42 & 5 \\
$\mathrm{C}(6)$ & 71 & 44 & 57 & 25 & 0 & 18 \\
$\mathrm{C}(7)$ & 38 & 47 & 28 & 18 & 12 & 22 \\
$\mathrm{C}(8)$ & 42 & 52 & 31 & 17 & 8 & 32 \\
$\mathrm{C}(9)$ & 31 & 51 & 129 & 6 & 2 & -5 \\
$\mathrm{C}(10)$ & 44 & 91 & 63 & 35 & -4 & 25 \\
$\mathrm{C}(11)$ & 58 & 75 & 76 & 32 & 39 & 36 \\
$\mathrm{C}(12)$ & 70 & 35 & 78 & 16 & 11 & 28 \\
$\mathrm{C}(13)$ & 40 & 59 & 49 & 1 & 5 & 1 \\
$\mathrm{C}(14)$ & 65 & 83 & 28 & 1 & 6 & 16 \\
$\mathrm{C}(15)$ & 34 & 52 & 47 & 9 & -1 & -5 \\
$\mathrm{C}(16)$ & 49 & 39 & 46 & -3 & 2 & 11 \\
$\mathrm{C}(17)$ & 54 & 43 & 43 & 21 & -12 & 4 \\
$\mathrm{C}(18)$ & 32 & 46 & 93 & 3 & 9 & 24 \\
$\mathrm{C}(19)$ & 57 & 62 & 124 & 54 & 22 & 1 \\
$\mathrm{C}(20)$ & 45 & 45 & 59 & -3 & -19 & 2 \\
$\mathrm{C}(21)$ & 95 & 66 & 57 & 30 & 19 & 57 \\
$\mathrm{C}(22)$ & 99 & 76 & 26 & 21 & 15 & 42 \\
$\mathrm{C}(23)$ & 39 & 39 & 52 & 8 & 2 & -7 \\
$\mathrm{C}(24)$ & 99 & 30 & 36 & -5 & 18 & 15 \\
$\mathrm{C}(25)$ & 77 & 59 & 85 & 42 & 33 & -1 \\
$\mathrm{C}(26)$ & 40 & 62 & 65 & 23 & 19 & 14 \\
$\mathrm{C}(27)$ & 38 & 49 & 27 & 2 & 0 & 24 \\
$\mathrm{C}(28)$ & 36 & 43 & 27 & 12 & -5 & 14 \\
$\mathrm{C}(29)$ & 36 & 35 & 46 & 14 & 3 & 2 \\
$\mathrm{C}(30)$ & 54 & 31 & 28 & 2 & -2 & 20 \\
$\mathrm{C}(31)$ & 49 & 49 & 43 & 18 & -16 & 9 \\
$\mathrm{C}(32)$ & 36 & 46 & 87 & 8 & 5 & 15 \\
$\mathrm{C}(33)$ & 71 & 37 & 48 & 14 & -35 & -2 \\
$\mathrm{C}(34)$ & 52 & 33 & 66 & 20 & -2 & -10 \\
$\mathrm{C}(35)$ & 32 & 35 & 70 & 13 & 4 & 0 \\
$\mathrm{C}(36)$ & 68 & 60 & 71 & 25 & -29 & 16 \\
$\mathrm{C}(37)$ & 41 & 58 & 56 & -1 & 11 & 15 \\
$\mathrm{C}(38)$ & 80 & 33 & 101 & 16 & -12 & 24 \\
$\mathrm{C}(39)$ & 65 & 60 & 158 & 27 & 58 & -9 \\
$\mathrm{C}(40)$ & 95 & 42 & 81 & -18 & -32 & 28 \\
$\mathrm{C}(41)$ & 73 & 39 & 117 & 13 & -31 & 4 \\
& & & & & &
\end{tabular}




\begin{tabular}{|c|c|c|c|c|c|c|}
\hline $\mathrm{C}(42)$ & 66 & 59 & 84 & 26 & -34 & 4 \\
\hline $\mathrm{C}(43)$ & 49 & 50 & 48 & 22 & 10 & 20 \\
\hline $\mathrm{C}(44)$ & 48 & 61 & 77 & 36 & 25 & 5 \\
\hline$C(45)$ & 78 & 82 & 32 & 14 & 21 & 35 \\
\hline$C(46)$ & 50 & 51 & 59 & 1 & 16 & 26 \\
\hline$C(47)$ & 33 & 37 & 26 & 6 & 9 & 20 \\
\hline$C(48)$ & 31 & 29 & 31 & 16 & 8 & 16 \\
\hline C(49) & 33 & 31 & 66 & 5 & 15 & 3 \\
\hline$C(50)$ & 58 & 38 & 44 & -4 & 15 & 12 \\
\hline $\mathrm{C}(51)$ & 54 & 41 & 62 & 24 & 4 & 15 \\
\hline$C(52)$ & 28 & 52 & 89 & 4 & 6 & 17 \\
\hline$C(53)$ & 44 & 39 & 58 & 15 & 5 & -5 \\
\hline $\mathrm{C}(54)$ & 39 & 66 & 46 & 5 & -5 & 1 \\
\hline$C(55)$ & 31 & 47 & 77 & 3 & 3 & -7 \\
\hline$C(56)$ & 43 & 87 & 66 & 41 & 5 & 29 \\
\hline$C(57)$ & 45 & 45 & 51 & 20 & 21 & 22 \\
\hline $\mathrm{C}(58)$ & 35 & 52 & 58 & 10 & 9 & -12 \\
\hline$C(59)$ & 64 & 51 & 34 & 4 & 6 & 16 \\
\hline$C(60)$ & 72 & 32 & 50 & 14 & 12 & 27 \\
\hline $\mathrm{C}(61)$ & 36 & 81 & 68 & -14 & -3 & -4 \\
\hline $\mathrm{C}(62)$ & 73 & 86 & 76 & -55 & 9 & -4 \\
\hline$C(63)$ & 128 & 181 & 115 & 111 & 68 & 75 \\
\hline$C(64)$ & 52 & 85 & 59 & 17 & 25 & 24 \\
\hline $\mathrm{C}(65)$ & 86 & 225 & 229 & 199 & 19 & 58 \\
\hline$C(66)$ & 222 & 50 & 107 & 26 & -12 & -2 \\
\hline$C(67)$ & 56 & 64 & 25 & 12 & 2 & 39 \\
\hline $\mathrm{C}(68)$ & 60 & 60 & 43 & 24 & 16 & 34 \\
\hline $\mathrm{C}(69)$ & 56 & 45 & 70 & 6 & 8 & -7 \\
\hline$C(70)$ & 39 & 102 & 53 & 34 & -1 & 18 \\
\hline $\mathrm{C}(71)$ & 57 & 54 & 44 & 7 & 17 & 32 \\
\hline$C(72)$ & 72 & 42 & 80 & 25 & -11 & 8 \\
\hline$C(73)$ & 45 & 97 & 57 & -12 & 12 & -14 \\
\hline$C(74)$ & 71 & 92 & 24 & -5 & -1 & 34 \\
\hline$C(75)$ & 33 & 63 & 80 & 11 & 5 & 1 \\
\hline$C(76)$ & 71 & 52 & 45 & 13 & -16 & 10 \\
\hline$C(77)$ & 53 & 54 & 53 & -11 & 11 & 6 \\
\hline $\mathrm{C}(78)$ & 38 & 33 & 167 & -4 & 1 & 17 \\
\hline$C(79)$ & 58 & 46 & 136 & 23 & 6 & 3 \\
\hline$C(80)$ & 74 & 60 & 84 & -12 & -45 & 22 \\
\hline $\mathrm{C}(81)$ & 35 & 70 & 48 & 28 & -2 & 3 \\
\hline $\mathrm{C}(82)$ & 35 & 66 & 44 & -1 & -2 & 14 \\
\hline$C(83)$ & 50 & 80 & 66 & -13 & 16 & 34 \\
\hline C(84) & 61 & 74 & 47 & 2 & 19 & 25 \\
\hline$C(85)$ & 60 & 44 & 63 & 15 & -9 & 13 \\
\hline$C(86)$ & 55 & 50 & 54 & 6 & -7 & 8 \\
\hline$C(87)$ & 49 & 48 & 80 & 24 & 15 & 0 \\
\hline
\end{tabular}




\begin{tabular}{|c|c|c|c|c|c|c|}
\hline $\mathrm{C}(88)$ & 78 & 96 & 60 & 33 & 30 & 28 \\
\hline C(89) & 134 & 157 & 183 & -129 & 135 & -67 \\
\hline$C(90)$ & 106 & 78 & 155 & -52 & 100 & -36 \\
\hline $\mathrm{C}(91)$ & 78 & 62 & 42 & -12 & -12 & 13 \\
\hline$C(92)$ & 45 & 36 & 64 & -6 & 1 & 3 \\
\hline $\mathrm{C}(93)$ & 39 & 49 & 38 & -2 & 13 & 2 \\
\hline$C(94)$ & 31 & 42 & 33 & 6 & -1 & -1 \\
\hline C(95) & 35 & 40 & 49 & 19 & 4 & 4 \\
\hline$C(96)$ & 46 & 45 & 23 & 9 & 10 & 0 \\
\hline C(97) & 54 & 42 & 41 & 2 & 12 & 11 \\
\hline $\mathrm{C}(98)$ & 63 & 43 & 59 & 3 & 13 & 22 \\
\hline C(99) & 62 & 49 & 42 & -5 & 14 & -1 \\
\hline$C(100)$ & 50 & 43 & 74 & -7 & 11 & 5 \\
\hline$C(101)$ & 34 & 65 & 69 & -11 & 19 & 9 \\
\hline$C(102)$ & 40 & 47 & 65 & -10 & 30 & 0 \\
\hline$C(103)$ & 59 & 38 & 52 & 2 & 22 & 9 \\
\hline C(104) & 64 & 47 & 47 & 12 & 25 & 30 \\
\hline $\mathrm{C}(105)$ & 101 & 259 & 144 & -124 & -14 & 99 \\
\hline$C(106)$ & 124 & 323 & 120 & -89 & 6 & 137 \\
\hline$C(107)$ & 44 & 214 & 95 & 70 & 18 & -1 \\
\hline$C(108)$ & 47 & 224 & 101 & 120 & -22 & -50 \\
\hline C(109) & 144 & 42 & 159 & 47 & -86 & -15 \\
\hline$C(110)$ & 151 & 30 & 118 & -14 & -91 & 17 \\
\hline $\mathrm{C}(111)$ & 197 & 143 & 43 & -7 & 3 & 128 \\
\hline $\mathrm{C}(112)$ & 133 & 265 & 48 & -6 & 26 & 140 \\
\hline$C(113)$ & 26 & 399 & 116 & 92 & 25 & 11 \\
\hline $\mathrm{C}(114)$ & 92 & 237 & 96 & 117 & -48 & -83 \\
\hline$C(115)$ & 152 & 51 & 180 & 51 & -123 & -43 \\
\hline$C(116)$ & 121 & 54 & 194 & -50 & -100 & 31 \\
\hline$C(117)$ & 50 & 63 & 37 & 11 & 2 & -2 \\
\hline$C(118)$ & 52 & 49 & 37 & 9 & -1 & 5 \\
\hline$C(119)$ & 42 & 45 & 49 & 0 & -3 & 5 \\
\hline$C(120)$ & 44 & 49 & 40 & -2 & -8 & 7 \\
\hline$C(121)$ & 38 & 82 & 64 & 3 & 2 & 26 \\
\hline$C(122)$ & 49 & 102 & 75 & -2 & 11 & 42 \\
\hline$C(123)$ & 68 & 148 & 49 & -1 & 17 & 51 \\
\hline$C(124)$ & 116 & 72 & 71 & 18 & 18 & 14 \\
\hline$C(125)$ & 132 & 72 & 226 & -84 & 114 & -31 \\
\hline$C(126)$ & 101 & 75 & 96 & -42 & -2 & 8 \\
\hline$C(127)$ & 137 & 61 & 63 & 1 & -18 & 49 \\
\hline$C(128)$ & 101 & 85 & 45 & 3 & -8 & 46 \\
\hline$C(129)$ & 94 & 56 & 77 & 16 & -1 & 39 \\
\hline$C(130)$ & 99 & 55 & 65 & 7 & 18 & 53 \\
\hline$C(131)$ & 38 & 102 & 57 & 2 & 6 & 23 \\
\hline$C(132)$ & 33 & 95 & 53 & 14 & -5 & 7 \\
\hline$C(133)$ & 101 & 48 & 48 & 7 & -2 & -12 \\
\hline
\end{tabular}




\begin{tabular}{|c|c|c|c|c|c|c|}
\hline $\mathrm{C}(134)$ & 100 & 26 & 61 & 15 & 22 & -2 \\
\hline$C(135)$ & 105 & 56 & 63 & 28 & -10 & 34 \\
\hline$C(136)$ & 90 & 59 & 72 & 5 & -13 & 53 \\
\hline$C(137)$ & 40 & 93 & 59 & 15 & 3 & 31 \\
\hline$C(138)$ & 37 & 75 & 63 & 14 & -14 & 10 \\
\hline C(139) & 93 & 33 & 39 & 5 & -5 & -12 \\
\hline$C(140)$ & 62 & 49 & 70 & 29 & 6 & -4 \\
\hline$C(141)$ & 33 & 28 & 39 & 2 & 12 & 11 \\
\hline$C(142)$ & 32 & 57 & 86 & 28 & 10 & 19 \\
\hline$C(143)$ & 55 & 34 & 36 & 10 & 17 & 15 \\
\hline$C(144)$ & 77 & 63 & 28 & 7 & 7 & 46 \\
\hline$C(145)$ & 45 & 121 & 24 & -15 & -4 & 40 \\
\hline$C(146)$ & 43 & 77 & 42 & -4 & 5 & 11 \\
\hline C(147) & 40 & 31 & 49 & -1 & 15 & 0 \\
\hline C(148) & 36 & 77 & 37 & -7 & 3 & 1 \\
\hline C(149) & 54 & 113 & 31 & 3 & -1 & 32 \\
\hline$C(150)$ & 87 & 73 & 35 & 9 & 10 & 37 \\
\hline $\mathrm{C}(151)$ & 38 & 35 & 69 & 6 & 10 & 10 \\
\hline$C(152)$ & 32 & 76 & 218 & 24 & 14 & 25 \\
\hline$C(153)$ & 71 & 40 & 56 & 4 & 28 & 10 \\
\hline$C(154)$ & 56 & 33 & 58 & 2 & 24 & 5 \\
\hline$C(155)$ & 56 & 90 & 56 & -7 & 3 & 49 \\
\hline$C(156)$ & 45 & 72 & 57 & 5 & 16 & -3 \\
\hline$C(157)$ & 50 & 48 & 73 & 18 & -6 & -20 \\
\hline $\mathrm{C}(158)$ & 103 & 34 & 65 & 13 & -1 & 20 \\
\hline$C(159)$ & 96 & 39 & 58 & 26 & -3 & 30 \\
\hline $\mathrm{C}(160)$ & 53 & 65 & 72 & 11 & -3 & 29 \\
\hline$C(161)$ & 46 & 35 & 41 & -1 & 19 & 7 \\
\hline$C(162)$ & 44 & 84 & 76 & 16 & 2 & 19 \\
\hline$C(163)$ & 46 & 48 & 55 & 1 & 2 & 3 \\
\hline$C(164)$ & 68 & 78 & 56 & -19 & 9 & 17 \\
\hline$C(165)$ & 81 & 98 & 56 & -17 & -9 & 60 \\
\hline$C(166)$ & 149 & 64 & 37 & 12 & 15 & 56 \\
\hline$C(167)$ & 82 & 44 & 58 & 20 & 27 & 32 \\
\hline$C(168)$ & 57 & 78 & 44 & 26 & 11 & 47 \\
\hline$C(169)$ & 56 & 74 & 56 & 22 & -2 & 33 \\
\hline$C(170)$ & 77 & 35 & 52 & 14 & 1 & 13 \\
\hline $\mathrm{C}(171)$ & 91 & 28 & 55 & 3 & -3 & 7 \\
\hline$C(172)$ & 36 & 49 & 75 & 16 & -3 & -12 \\
\hline$C(173)$ & 31 & 71 & 61 & 20 & 13 & -9 \\
\hline$C(174)$ & 33 & 48 & 51 & 3 & 8 & 1 \\
\hline$C(175)$ & 37 & 103 & 98 & 23 & -6 & 0 \\
\hline$C(176)$ & 68 & 39 & 40 & 6 & 14 & 6 \\
\hline$C(177)$ & 122 & 53 & 49 & 23 & 23 & 40 \\
\hline$C(178)$ & 66 & 63 & 52 & 6 & 13 & 21 \\
\hline $\mathrm{C}(179)$ & 43 & 49 & 59 & 4 & 12 & 2 \\
\hline
\end{tabular}




\begin{tabular}{|c|c|c|c|c|c|c|}
\hline$C(180)$ & 34 & 47 & 51 & 4 & 8 & 8 \\
\hline $\mathrm{Hf}(1)$ & 36 & 34 & 21 & 8 & 2 & 9 \\
\hline $\operatorname{Hf}(2)$ & 35 & 24 & 25 & 7 & 5 & 5 \\
\hline $\operatorname{Hf}(3)$ & 26 & 31 & 22 & 5 & 0 & 4 \\
\hline $\mathrm{Hf}(4)$ & 32 & 34 & 24 & 4 & 0 & 6 \\
\hline $\mathrm{K}(1)$ & 38 & 43 & 27 & 7 & 6 & 7 \\
\hline$K(2)$ & 36 & 32 & 27 & 3 & 3 & 3 \\
\hline $\mathrm{K}(3)$ & 37 & 27 & 45 & 10 & 11 & 13 \\
\hline $\mathrm{K}(4)$ & 32 & 30 & 66 & 13 & 6 & 11 \\
\hline $\mathrm{K}(5)$ & 30 & 29 & 56 & 11 & 7 & 11 \\
\hline $\mathrm{N}(1)$ & 90 & 67 & 21 & 12 & 9 & 41 \\
\hline $\mathrm{N}(2)$ & 60 & 48 & 34 & 25 & 18 & 13 \\
\hline $\mathrm{N}(3)$ & 49 & 47 & 32 & 6 & -18 & -2 \\
\hline N(4) & 63 & 41 & 32 & 16 & 11 & 20 \\
\hline $\mathrm{N}(5)$ & 43 & 23 & 38 & 1 & -4 & 5 \\
\hline $\mathrm{N}(6)$ & 40 & 42 & 47 & 18 & 21 & 3 \\
\hline $\mathrm{N}(7)$ & 35 & 45 & 36 & 1 & -10 & 1 \\
\hline $\mathrm{N}(8)$ & 27 & 41 & 34 & 18 & 6 & 4 \\
\hline N(9) & 40 & 53 & 30 & 2 & 10 & 16 \\
\hline $\mathrm{N}(10)$ & 41 & 52 & 30 & -2 & 0 & -7 \\
\hline $\mathrm{N}(11)$ & 39 & 73 & 46 & 21 & 8 & 0 \\
\hline $\mathrm{N}(12)$ & 134 & 78 & 38 & 22 & 19 & 65 \\
\hline $\mathrm{O}(1)$ & 36 & 42 & 46 & 17 & 2 & 5 \\
\hline $\mathrm{O}(2)$ & 39 & 69 & 45 & 9 & 11 & 19 \\
\hline $\mathrm{O}(3)$ & 66 & 38 & 47 & 2 & 0 & 18 \\
\hline $\mathrm{O}(4)$ & 50 & 49 & 40 & 10 & -3 & 3 \\
\hline $\mathrm{O}(5)$ & 63 & 62 & 64 & -20 & 30 & -20 \\
\hline $\mathrm{O}(6)$ & 47 & 46 & 53 & -3 & 18 & 3 \\
\hline $\mathrm{O}(7)$ & 36 & 34 & 39 & 5 & 8 & 3 \\
\hline $\mathrm{O}(8)$ & 29 & 36 & 34 & 12 & -2 & 4 \\
\hline $\mathrm{O}(9)$ & 49 & 45 & 31 & 15 & 9 & 11 \\
\hline $\mathrm{O}(10)$ & 52 & 38 & 54 & 2 & 4 & 7 \\
\hline $\mathrm{O}(11)$ & 43 & 44 & 50 & -2 & 9 & 3 \\
\hline $\mathrm{O}(12)$ & 48 & 43 & 51 & 6 & 20 & 11 \\
\hline $\mathrm{O}(13)$ & 121 & 65 & 170 & -52 & -78 & 59 \\
\hline $\mathrm{O}(14)$ & 52 & 215 & 47 & 13 & 14 & 45 \\
\hline $\mathrm{O}(15)$ & 61 & 75 & 103 & 58 & -36 & -19 \\
\hline $\mathrm{O}(16)$ & 136 & 52 & 51 & -15 & -45 & 46 \\
\hline $\mathrm{O}(17)$ & 51 & 169 & 58 & 57 & 20 & 36 \\
\hline $\mathrm{O}(18)$ & 66 & 76 & 93 & 52 & -36 & -21 \\
\hline $\mathrm{O}(19)$ & 55 & 47 & 37 & 9 & -10 & 2 \\
\hline $\mathrm{O}(20)$ & 36 & 41 & 39 & 4 & -7 & 6 \\
\hline $\mathrm{O}(21)$ & 51 & 57 & 45 & 0 & -6 & 20 \\
\hline $\mathrm{O}(22)$ & 47 & 101 & 76 & 18 & 7 & 32 \\
\hline $\mathrm{O}(23)$ & 131 & 72 & 85 & -10 & 47 & -2 \\
\hline $\mathrm{O}(24)$ & 104 & 98 & 71 & 5 & 9 & 51 \\
\hline
\end{tabular}




\begin{tabular}{|c|c|c|c|c|c|}
\hline $\mathrm{O}(25)$ & 93 & 44 & 37 & 14 & -2 \\
\hline $\mathrm{O}(26)$ & 67 & 74 & 37 & 9 & 7 \\
\hline $\mathrm{O}(27)$ & 55 & 53 & 39 & 2 & 5 \\
\hline $\mathrm{O}(28)$ & 101 & 34 & 43 & 10 & -1 \\
\hline $\mathrm{O}(29)$ & 57 & 67 & 55 & 4 & 2 \\
\hline $\mathrm{O}(30)$ & 56 & 57 & 41 & 13 & 1 \\
\hline $\mathrm{O}(31)$ & 39 & 56 & 43 & 2 & 7 \\
\hline $\mathrm{O}(32)$ & 70 & 36 & 44 & 10 & 4 \\
\hline $\mathrm{O}(33)$ & 66 & 63 & 36 & 3 & -4 \\
\hline $\mathrm{O}(34)$ & 35 & 61 & 34 & 16 & 8 \\
\hline $\mathrm{O}(35)$ & 59 & 32 & 43 & 5 & -4 \\
\hline $\mathrm{O}(36)$ & 72 & 38 & 36 & 8 & 5 \\
\hline $\operatorname{Si}(1)$ & 23 & 30 & 27 & 9 & 3 \\
\hline $\operatorname{Si}(2)$ & 26 & 45 & 57 & 20 & 10 \\
\hline $\operatorname{Si}(3)$ & 40 & 37 & 36 & 2 & 7 \\
\hline $\operatorname{Si}(4)$ & 24 & 33 & 24 & 8 & 3 \\
\hline $\operatorname{Si}(5)$ & 28 & 34 & 27 & 8 & -4 \\
\hline $\operatorname{Si}(6)$ & 25 & 32 & 52 & 12 & 2 \\
\hline $\mathrm{Si}(7)$ & 28 & 21 & 30 & 5 & 0 \\
\hline $\operatorname{Si}(8)$ & 29 & 26 & 30 & 9 & -2 \\
\hline $\operatorname{Si}(9)$ & 30 & 24 & 51 & 6 & -7 \\
\hline $\operatorname{Si}(10)$ & 28 & 23 & 27 & 6 & 2 \\
\hline $\operatorname{Si}(11)$ & 30 & 33 & 41 & 8 & 0 \\
\hline $\mathrm{Si}(12)$ & 41 & 24 & 66 & 5 & 8 \\
\hline $\mathrm{Si}(13)$ & 21 & 25 & 29 & 7 & 3 \\
\hline $\mathrm{Si}(14)$ & 26 & 26 & 37 & 5 & 2 \\
\hline $\operatorname{Si}(15)$ & 22 & 30 & 38 & 9 & 4 \\
\hline $\operatorname{Si}(16)$ & 18 & 22 & 29 & 9 & 4 \\
\hline $\operatorname{Si}(17)$ & 21 & 39 & 46 & 19 & 6 \\
\hline $\mathrm{Si}(18)$ & 33 & 25 & 31 & 4 & 6 \\
\hline $\operatorname{Si}(19)$ & 38 & 41 & 27 & 11 & 4 \\
\hline $\operatorname{Si}(20)$ & 40 & 41 & 40 & 13 & 9 \\
\hline $\operatorname{Si}(21)$ & 35 & 50 & 38 & 1 & 2 \\
\hline $\operatorname{Si}(22)$ & 33 & 41 & 30 & 1 & -1 \\
\hline $\operatorname{Si}(23)$ & 25 & 41 & 44 & 1 & -2 \\
\hline $\operatorname{Si}(24)$ & 38 & 25 & 68 & -7 & -11 \\
\hline
\end{tabular}


Table S20. Hydrogen coordinates $\left(\times 10^{4}\right)$ and isotropic displacement parameters $\left(\AA^{2} \times\right.$ $10^{3}$ ) for 4 .

\begin{tabular}{|c|c|c|c|c|}
\hline & $\mathrm{x}$ & $\mathrm{y}$ & $\mathrm{Z}$ & $\mathrm{U}(\mathrm{eq})$ \\
\hline $\mathrm{H}(1 \mathrm{~A})$ & 215 & 8669 & 7271 & 124 \\
\hline $\mathrm{H}(1 \mathrm{~B})$ & 435 & 8060 & 7178 & 124 \\
\hline $\mathrm{H}(1 \mathrm{C})$ & -704 & 8143 & 7235 & 124 \\
\hline $\mathrm{H}(2 \mathrm{~A})$ & -1672 & 8435 & 6855 & 90 \\
\hline $\mathrm{H}(2 \mathrm{~B})$ & -1155 & 8657 & 6558 & 90 \\
\hline $\mathrm{H}(2 \mathrm{C})$ & -818 & 8993 & 6916 & 90 \\
\hline $\mathrm{H}(3 \mathrm{~A})$ & 2362 & 9054 & 7027 & 114 \\
\hline $\mathrm{H}(3 \mathrm{~B})$ & 1855 & 8977 & 6671 & 114 \\
\hline $\mathrm{H}(3 \mathrm{C})$ & 3040 & 8971 & 6726 & 114 \\
\hline $\mathrm{H}(4 \mathrm{~A})$ & 3490 & 8161 & 6934 & 114 \\
\hline $\mathrm{H}(4 \mathrm{~B})$ & 2597 & 7645 & 6955 & 114 \\
\hline $\mathrm{H}(4 \mathrm{C})$ & 2720 & 8210 & 7213 & 114 \\
\hline $\mathrm{H}(5 \mathrm{~A})$ & -1127 & 6535 & 6624 & 116 \\
\hline $\mathrm{H}(5 \mathrm{~B})$ & -1210 & 7154 & 6776 & 116 \\
\hline $\mathrm{H}(5 \mathrm{C})$ & -622 & 6817 & 6977 & 116 \\
\hline $\mathrm{H}(6 \mathrm{~A})$ & 1097 & 6630 & 6795 & 82 \\
\hline $\mathrm{H}(6 \mathrm{~B})$ & 1452 & 6809 & 6469 & 82 \\
\hline $\mathrm{H}(6 \mathrm{C})$ & 473 & 6324 & 6462 & 82 \\
\hline $\mathrm{H}(7 \mathrm{~A})$ & 1823 & 8261 & 5633 & 41 \\
\hline $\mathrm{H}(7 \mathrm{~B})$ & 1552 & 7693 & 5374 & 41 \\
\hline $\mathrm{H}(8 \mathrm{~A})$ & -214 & 7565 & 5481 & 44 \\
\hline $\mathrm{H}(8 \mathrm{~B})$ & 118 & 8173 & 5408 & 44 \\
\hline $\mathrm{H}(9 \mathrm{~A})$ & 3845 & 8447 & 6278 & 111 \\
\hline $\mathrm{H}(9 \mathrm{~B})$ & 3846 & 8501 & 5910 & 111 \\
\hline $\mathrm{H}(9 \mathrm{C})$ & 4791 & 8330 & 6075 & 111 \\
\hline $\mathrm{H}(10 \mathrm{~A})$ & 4632 & 7178 & 6229 & 93 \\
\hline $\mathrm{H}(10 \mathrm{~B})$ & 3545 & 6763 & 6199 & 93 \\
\hline $\mathrm{H}(10 \mathrm{C})$ & 3771 & 7324 & 6462 & 93 \\
\hline $\mathrm{H}(11 \mathrm{~A})$ & 3645 & 7438 & 5368 & 96 \\
\hline $\mathrm{H}(11 \mathrm{~B})$ & 3702 & 6868 & 5474 & 96 \\
\hline $\mathrm{H}(11 \mathrm{C})$ & 4662 & 7376 & 5553 & 96 \\
\hline $\mathrm{H}(12 \mathrm{~A})$ & 1274 & 5638 & 5650 & 87 \\
\hline $\mathrm{H}(12 \mathrm{~B})$ & 1515 & 5997 & 6006 & 87 \\
\hline $\mathrm{H}(12 \mathrm{C})$ & 2293 & 6107 & 5737 & 87 \\
\hline $\mathrm{H}(13 \mathrm{~A})$ & -990 & 6554 & 5669 & 78 \\
\hline $\mathrm{H}(13 \mathrm{~B})$ & -633 & 6362 & 5984 & 78 \\
\hline $\mathrm{H}(13 \mathrm{C})$ & -845 & 5934 & 5648 & 78 \\
\hline $\mathrm{H}(14 \mathrm{~A})$ & 632 & 6054 & 5105 & 90 \\
\hline $\mathrm{H}(14 \mathrm{~B})$ & 1549 & 6581 & 5145 & 90 \\
\hline $\mathrm{H}(14 \mathrm{C})$ & 390 & 6655 & 5123 & 90 \\
\hline $\mathrm{H}(15 \mathrm{~A})$ & 1939 & 9830 & 5981 & 70 \\
\hline
\end{tabular}




\begin{tabular}{|c|c|c|c|c|}
\hline $\mathrm{H}(15 \mathrm{~B})$ & 1959 & 9230 & 5779 & 70 \\
\hline $\mathrm{H}(15 \mathrm{C})$ & 2076 & 9344 & 6162 & 70 \\
\hline $\mathrm{H}(16 \mathrm{~A})$ & 172 & 9619 & 6544 & 69 \\
\hline $\mathrm{H}(16 \mathrm{~B})$ & -814 & 9669 & 6338 & 69 \\
\hline $\mathrm{H}(16 \mathrm{C})$ & 247 & 10093 & 6338 & 69 \\
\hline $\mathrm{H}(17 \mathrm{~A})$ & -103 & 9819 & 5629 & 70 \\
\hline $\mathrm{H}(17 \mathrm{~B})$ & -1003 & 9282 & 5584 & 70 \\
\hline $\mathrm{H}(17 \mathrm{C})$ & 44 & 9247 & 5418 & 70 \\
\hline $\mathrm{H}(18 \mathrm{~A})$ & -2409 & 8967 & 5951 & 84 \\
\hline $\mathrm{H}(18 \mathrm{~B})$ & -2720 & 8729 & 6265 & 84 \\
\hline $\mathrm{H}(18 \mathrm{C})$ & -3455 & 8527 & 5943 & 84 \\
\hline $\mathrm{H}(19 \mathrm{~A})$ & -3383 & 7396 & 6080 & 117 \\
\hline $\mathrm{H}(19 \mathrm{~B})$ & -2403 & 7545 & 6331 & 117 \\
\hline $\mathrm{H}(19 \mathrm{C})$ & -2429 & 7111 & 6002 & 117 \\
\hline $\mathrm{H}(20 \mathrm{~A})$ & -2076 & 7498 & 5370 & 80 \\
\hline $\mathrm{H}(20 \mathrm{~B})$ & -2274 & 8095 & 5344 & 80 \\
\hline $\mathrm{H}(20 \mathrm{C})$ & -3177 & 7638 & 5431 & 80 \\
\hline $\mathrm{H}(21 \mathrm{~A})$ & 8657 & 10381 & 8066 & 97 \\
\hline $\mathrm{H}(21 \mathrm{~B})$ & 7837 & 10135 & 8294 & 97 \\
\hline $\mathrm{H}(21 \mathrm{C})$ & 7634 & 9952 & 7913 & 97 \\
\hline $\mathrm{H}(22 \mathrm{~A})$ & 8105 & 9107 & 7657 & 93 \\
\hline $\mathrm{H}(22 \mathrm{~B})$ & 9270 & 9088 & 7741 & 93 \\
\hline $\mathrm{H}(22 \mathrm{C})$ & 8999 & 9617 & 7628 & 93 \\
\hline $\mathrm{H}(23 \mathrm{~A})$ & 7493 & 7995 & 8148 & 69 \\
\hline $\mathrm{H}(23 \mathrm{~B})$ & 7723 & 8438 & 8478 & 69 \\
\hline $\mathrm{H}(23 \mathrm{C})$ & 8058 & 7857 & 8451 & 69 \\
\hline $\mathrm{H}(24 \mathrm{~A})$ & 9410 & 7661 & 8053 & 84 \\
\hline $\mathrm{H}(24 \mathrm{~B})$ & 10032 & 8177 & 7924 & 84 \\
\hline $\mathrm{H}(24 \mathrm{C})$ & 8886 & 7900 & 7782 & 84 \\
\hline $\mathrm{H}(25 \mathrm{~A})$ & 11799 & 10165 & 8238 & 107 \\
\hline $\mathrm{H}(25 \mathrm{~B})$ & 10689 & 10084 & 8062 & 107 \\
\hline $\mathrm{H}(25 \mathrm{C})$ & 11507 & 9757 & 7897 & 107 \\
\hline $\mathrm{H}(26 \mathrm{~A})$ & 11770 & 8922 & 8071 & 80 \\
\hline $\mathrm{H}(26 \mathrm{~B})$ & 11334 & 8793 & 8400 & 80 \\
\hline $\mathrm{H}(26 \mathrm{C})$ & 12248 & 9312 & 8405 & 80 \\
\hline $\mathrm{H}(27 \mathrm{~A})$ & 9585 & 9466 & 9617 & 44 \\
\hline $\mathrm{H}(27 \mathrm{~B})$ & 9939 & 9998 & 9461 & 44 \\
\hline $\mathrm{H}(28 \mathrm{~A})$ & 8154 & 9879 & 9490 & 40 \\
\hline $\mathrm{H}(28 \mathrm{~B})$ & 7980 & 9238 & 9326 & 40 \\
\hline $\mathrm{H}(29 \mathrm{~A})$ & 7904 & 8109 & 9038 & 59 \\
\hline $\mathrm{H}(29 \mathrm{~B})$ & 8111 & 8235 & 9420 & 59 \\
\hline $\mathrm{H}(29 \mathrm{C})$ & 8110 & 7633 & 9216 & 59 \\
\hline $\mathrm{H}(30 \mathrm{~A})$ & 10007 & 7357 & 8893 & 55 \\
\hline $\mathrm{H}(30 \mathrm{~B})$ & 10829 & 7842 & 8796 & 55 \\
\hline $\mathrm{H}(30 \mathrm{C})$ & 9683 & 7693 & 8636 & 55 \\
\hline $\mathrm{H}(31 \mathrm{~A})$ & 10107 & 7767 & 9605 & 70 \\
\hline
\end{tabular}




\begin{tabular}{|c|c|c|c|c|}
\hline $\mathrm{H}(31 \mathrm{~B})$ & 10242 & 8412 & 9752 & 70 \\
\hline $\mathrm{H}(31 \mathrm{C})$ & 11120 & 8190 & 9554 & 70 \\
\hline $\mathrm{H}(32 \mathrm{~A})$ & 12637 & 8812 & 8869 & 84 \\
\hline $\mathrm{H}(32 \mathrm{~B})$ & 12592 & 8638 & 9211 & 84 \\
\hline $\mathrm{H}(32 \mathrm{C})$ & 13476 & 9131 & 9153 & 84 \\
\hline $\mathrm{H}(33 \mathrm{~A})$ & 12082 & 9312 & 9787 & 81 \\
\hline $\mathrm{H}(33 \mathrm{~B})$ & 11622 & 9852 & 9792 & 81 \\
\hline $\mathrm{H}(33 \mathrm{C})$ & 12820 & 9883 & 9762 & 81 \\
\hline $\mathrm{H}(34 \mathrm{~A})$ & 13160 & 10253 & 9156 & 78 \\
\hline $\mathrm{H}(34 \mathrm{~B})$ & 12067 & 10417 & 9186 & 78 \\
\hline $\mathrm{H}(34 \mathrm{C})$ & 12326 & 10064 & 8857 & 78 \\
\hline $\mathrm{H}(35 \mathrm{~A})$ & 6041 & 8756 & 9018 & 70 \\
\hline $\mathrm{H}(35 \mathrm{~B})$ & 6025 & 8675 & 8636 & 70 \\
\hline $\mathrm{H}(35 \mathrm{C})$ & 5060 & 8826 & 8817 & 70 \\
\hline $\mathrm{H}(36 \mathrm{~A})$ & 4971 & 9813 & 8541 & 98 \\
\hline $\mathrm{H}(36 \mathrm{~B})$ & 5846 & 9664 & 8313 & 98 \\
\hline $\mathrm{H}(36 \mathrm{C})$ & 5965 & 10275 & 8522 & 98 \\
\hline $\mathrm{H}(37 \mathrm{~A})$ & 5173 & 9881 & 9269 & 79 \\
\hline $\mathrm{H}(37 \mathrm{~B})$ & 6027 & 10426 & 9272 & 79 \\
\hline $\mathrm{H}(37 \mathrm{C})$ & 6274 & 9955 & 9449 & 79 \\
\hline $\mathrm{H}(38 \mathrm{~A})$ & 7165 & 10973 & 8857 & 104 \\
\hline $\mathrm{H}(38 \mathrm{~B})$ & 8008 & 11265 & 8651 & 104 \\
\hline $\mathrm{H}(38 \mathrm{C})$ & 7921 & 11543 & 9017 & 104 \\
\hline $\mathrm{H}(39 \mathrm{~A})$ & 10345 & 11359 & 8843 & 144 \\
\hline H(39B) & 9884 & 10864 & 8549 & 144 \\
\hline $\mathrm{H}(39 \mathrm{C})$ & 10559 & 10756 & 8839 & 144 \\
\hline $\mathrm{H}(40 \mathrm{~A})$ & 9637 & 10891 & 9534 & 113 \\
\hline $\mathrm{H}(40 \mathrm{~B})$ & 8520 & 11025 & 9562 & 113 \\
\hline $\mathrm{H}(40 \mathrm{C})$ & 9444 & 11475 & 9480 & 113 \\
\hline $\mathrm{H}(41 \mathrm{~A})$ & 6940 & 3546 & 8121 & 118 \\
\hline $\mathrm{H}(41 \mathrm{~B})$ & 8089 & 3541 & 8235 & 118 \\
\hline $\mathrm{H}(41 \mathrm{C})$ & 7791 & 3526 & 7866 & 118 \\
\hline $\mathrm{H}(42 \mathrm{~A})$ & 7206 & 4415 & 7743 & 106 \\
\hline $\mathrm{H}(42 \mathrm{~B})$ & 7223 & 4891 & 8051 & 106 \\
\hline $\mathrm{H}(42 \mathrm{C})$ & 6434 & 4313 & 8015 & 106 \\
\hline $\mathrm{H}(43 \mathrm{~A})$ & 9266 & 6198 & 8539 & 68 \\
\hline $\mathrm{H}(43 \mathrm{~B})$ & 8316 & 5687 & 8450 & 68 \\
\hline $\mathrm{H}(43 \mathrm{C})$ & 8861 & 5957 & 8172 & 68 \\
\hline $\mathrm{H}(44 \mathrm{~A})$ & 10530 & 5869 & 8049 & 89 \\
\hline $\mathrm{H}(44 \mathrm{~B})$ & 11100 & 5460 & 8196 & 89 \\
\hline $\mathrm{H}(44 \mathrm{C})$ & 11021 & 6041 & 8411 & 89 \\
\hline $\mathrm{H}(45 \mathrm{~A})$ & 10783 & 4640 & 7763 & 91 \\
\hline $\mathrm{H}(45 \mathrm{~B})$ & 9569 & 4542 & 7779 & 91 \\
\hline $\mathrm{H}(45 \mathrm{C})$ & 10096 & 4031 & 7658 & 91 \\
\hline $\mathrm{H}(46 \mathrm{~A})$ & 10820 & 3599 & 8025 & 78 \\
\hline $\mathrm{H}(46 \mathrm{~B})$ & 11159 & 3917 & 8386 & 78 \\
\hline
\end{tabular}




\begin{tabular}{|c|c|c|c|c|}
\hline $\mathrm{H}(46 \mathrm{C})$ & 11701 & 4147 & 8095 & 78 \\
\hline $\mathrm{H}(47 \mathrm{~A})$ & 9667 & 4235 & 9522 & 36 \\
\hline $\mathrm{H}(47 \mathrm{~B})$ & 9999 & 4861 & 9476 & 36 \\
\hline $\mathrm{H}(48 \mathrm{~A})$ & 8195 & 4680 & 9542 & 33 \\
\hline $\mathrm{H}(48 \mathrm{~B})$ & 8012 & 4146 & 9257 & 33 \\
\hline $\mathrm{H}(49 \mathrm{~A})$ & 7835 & 3206 & 8795 & 66 \\
\hline $\mathrm{H}(49 \mathrm{~B})$ & 7983 & 3130 & 9157 & 66 \\
\hline $\mathrm{H}(49 \mathrm{C})$ & 7975 & 2624 & 8867 & 66 \\
\hline $\mathrm{H}(50 \mathrm{~A})$ & 9746 & 2401 & 8570 & 72 \\
\hline $\mathrm{H}(50 \mathrm{~B})$ & 10698 & 2876 & 8535 & 72 \\
\hline $\mathrm{H}(50 \mathrm{C})$ & 9603 & 2853 & 8364 & 72 \\
\hline $\mathrm{H}(51 \mathrm{~A})$ & 9935 & 2617 & 9276 & 74 \\
\hline $\mathrm{H}(51 \mathrm{~B})$ & 10048 & 3223 & 9490 & 74 \\
\hline $\mathrm{H}(51 \mathrm{C})$ & 10959 & 3071 & 9280 & 74 \\
\hline $\mathrm{H}(52 \mathrm{~A})$ & 12507 & 3729 & 8720 & 84 \\
\hline $\mathrm{H}(52 \mathrm{~B})$ & 12454 & 3617 & 9078 & 84 \\
\hline $\mathrm{H}(52 \mathrm{C})$ & 13395 & 4050 & 8989 & 84 \\
\hline $\mathrm{H}(53 \mathrm{~A})$ & 13226 & 5166 & 8887 & 72 \\
\hline $\mathrm{H}(53 \mathrm{~B})$ & 12169 & 5372 & 8895 & 72 \\
\hline $\mathrm{H}(53 \mathrm{C})$ & 12357 & 4911 & 8602 & 72 \\
\hline $\mathrm{H}(54 \mathrm{~A})$ & 13060 & 4880 & 9542 & 79 \\
\hline $\mathrm{H}(54 \mathrm{~B})$ & 12057 & 4496 & 9635 & 79 \\
\hline $\mathrm{H}(54 \mathrm{C})$ & 12024 & 5104 & 9581 & 79 \\
\hline $\mathrm{H}(55 \mathrm{~A})$ & 5955 & 3952 & 8969 & 83 \\
\hline $\mathrm{H}(55 \mathrm{~B})$ & 6029 & 4056 & 8611 & 83 \\
\hline $\mathrm{H}(55 \mathrm{C})$ & 5050 & 4154 & 8802 & 83 \\
\hline $\mathrm{H}(56 \mathrm{~A})$ & 5297 & 5360 & 8703 & 89 \\
\hline $\mathrm{H}(56 \mathrm{~B})$ & 6240 & 5281 & 8491 & 89 \\
\hline $\mathrm{H}(56 \mathrm{C})$ & 6370 & 5787 & 8789 & 89 \\
\hline $\mathrm{H}(57 \mathrm{~A})$ & 5159 & 5056 & 9369 & 65 \\
\hline $\mathrm{H}(57 \mathrm{~B})$ & 6077 & 5585 & 9452 & 65 \\
\hline $\mathrm{H}(57 \mathrm{C})$ & 6192 & 5023 & 9558 & 65 \\
\hline $\mathrm{H}(58 \mathrm{~A})$ & 10506 & 6121 & 9012 & 77 \\
\hline $\mathrm{H}(58 \mathrm{~B})$ & 10829 & 5841 & 9296 & 77 \\
\hline $\mathrm{H}(58 \mathrm{C})$ & 10736 & 6475 & 9370 & 77 \\
\hline $\mathrm{H}(59 \mathrm{~A})$ & 9301 & 6294 & 9900 & 75 \\
\hline $\mathrm{H}(59 \mathrm{~B})$ & 9388 & 5660 & 9847 & 75 \\
\hline $\mathrm{H}(59 \mathrm{C})$ & 8288 & 5818 & 9842 & 75 \\
\hline $\mathrm{H}(60 \mathrm{~A})$ & 8710 & 6820 & 9339 & 72 \\
\hline $\mathrm{H}(60 \mathrm{~B})$ & 7637 & 6405 & 9337 & 72 \\
\hline $\mathrm{H}(60 \mathrm{C})$ & 8188 & 6430 & 9010 & 72 \\
\hline $\mathrm{H}(61 \mathrm{~A})$ & 3190 & 4033 & 6682 & 102 \\
\hline $\mathrm{H}(61 \mathrm{~B})$ & 3111 & 3380 & 6619 & 102 \\
\hline $\mathrm{H}(61 \mathrm{C})$ & 3386 & 3742 & 6974 & 102 \\
\hline $\mathrm{H}(62 \mathrm{~A})$ & 2062 & 4113 & 7288 & 136 \\
\hline $\mathrm{H}(62 \mathrm{~B})$ & 918 & 3997 & 7131 & 136 \\
\hline
\end{tabular}




\begin{tabular}{|c|c|c|c|c|}
\hline $\mathrm{H}(62 \mathrm{C})$ & 1794 & 4436 & 7017 & 136 \\
\hline $\mathrm{H}(63 \mathrm{~A})$ & -426 & 2961 & 7351 & 184 \\
\hline $\mathrm{H}(63 \mathrm{~B})$ & -304 & 2389 & 7134 & 184 \\
\hline $\mathrm{H}(63 \mathrm{C})$ & -1400 & 2552 & 7150 & 184 \\
\hline $\mathrm{H}(64 \mathrm{~A})$ & -1896 & 3127 & 6877 & 95 \\
\hline $\mathrm{H}(64 \mathrm{~B})$ & -1177 & 3474 & 6661 & 95 \\
\hline $\mathrm{H}(64 \mathrm{C})$ & -1043 & 3652 & 7044 & 95 \\
\hline $\mathrm{H}(65 \mathrm{~A})$ & 1945 & 1908 & 7048 & 233 \\
\hline $\mathrm{H}(65 \mathrm{~B})$ & 2488 & 2528 & 7033 & 233 \\
\hline $\mathrm{H}(65 \mathrm{C})$ & 2538 & 2033 & 6741 & 233 \\
\hline $\mathrm{H}(66 \mathrm{~A})$ & 558 & 1418 & 6535 & 194 \\
\hline $\mathrm{H}(66 \mathrm{~B})$ & -285 & 1749 & 6676 & 194 \\
\hline $\mathrm{H}(66 \mathrm{C})$ & 416 & 1528 & 6912 & 194 \\
\hline $\mathrm{H}(67 \mathrm{~A})$ & -184 & 2587 & 5566 & 53 \\
\hline $\mathrm{H}(67 \mathrm{~B})$ & 62 & 3201 & 5498 & 53 \\
\hline $\mathrm{H}(68 \mathrm{~A})$ & 1772 & 3337 & 5699 & 59 \\
\hline $\mathrm{H}(68 \mathrm{~B})$ & 1525 & 2769 & 5440 & 59 \\
\hline $\mathrm{H}(69 \mathrm{~A})$ & 4207 & 3584 & 6179 & 91 \\
\hline $\mathrm{H}(69 \mathrm{~B})$ & 3656 & 3556 & 5835 & 91 \\
\hline $\mathrm{H}(69 \mathrm{C})$ & 4765 & 3413 & 5860 & 91 \\
\hline $\mathrm{H}(70 \mathrm{~A})$ & 3846 & 1991 & 6275 & 92 \\
\hline $\mathrm{H}(70 \mathrm{~B})$ & 4140 & 2613 & 6473 & 92 \\
\hline $\mathrm{H}(70 \mathrm{C})$ & 4889 & 2387 & 6220 & 92 \\
\hline $\mathrm{H}(71 \mathrm{~A})$ & 4583 & 2315 & 5517 & 74 \\
\hline $\mathrm{H}(71 \mathrm{~B})$ & 3510 & 2343 & 5350 & 74 \\
\hline $\mathrm{H}(71 \mathrm{C})$ & 3610 & 1827 & 5509 & 74 \\
\hline $\mathrm{H}(72 \mathrm{~A})$ & 1471 & 730 & 5788 & 96 \\
\hline $\mathrm{H}(72 \mathrm{~B})$ & 1724 & 1141 & 6129 & 96 \\
\hline $\mathrm{H}(72 \mathrm{C})$ & 2476 & 1211 & 5849 & 96 \\
\hline $\mathrm{H}(73 \mathrm{~A})$ & -851 & 1582 & 5712 & 111 \\
\hline $\mathrm{H}(73 \mathrm{~B})$ & -538 & 1448 & 6050 & 111 \\
\hline $\mathrm{H}(73 \mathrm{C})$ & -675 & 979 & 5732 & 111 \\
\hline $\mathrm{H}(74 \mathrm{~A})$ & 733 & 1078 & 5202 & 93 \\
\hline $\mathrm{H}(74 \mathrm{~B})$ & 1683 & 1585 & 5227 & 93 \\
\hline $\mathrm{H}(74 \mathrm{C})$ & 542 & 1685 & 5204 & 93 \\
\hline $\mathrm{H}(75 \mathrm{~A})$ & 1871 & 4381 & 5915 & 91 \\
\hline $\mathrm{H}(75 \mathrm{~B})$ & 1964 & 4373 & 6289 & 91 \\
\hline $\mathrm{H}(75 \mathrm{C})$ & 1762 & 4913 & 6173 & 91 \\
\hline $\mathrm{H}(76 \mathrm{~A})$ & -289 & 4862 & 5783 & 85 \\
\hline $\mathrm{H}(76 \mathrm{~B})$ & -1145 & 4304 & 5716 & 85 \\
\hline $\mathrm{H}(76 \mathrm{C})$ & -86 & 4322 & 5550 & 85 \\
\hline $\mathrm{H}(77 \mathrm{~A})$ & 110 & 4598 & 6682 & 85 \\
\hline $\mathrm{H}(77 \mathrm{~B})$ & -998 & 4521 & 6505 & 85 \\
\hline $\mathrm{H}(77 \mathrm{C})$ & -122 & 5042 & 6482 & 85 \\
\hline $\mathrm{H}(78 \mathrm{~A})$ & -2654 & 3836 & 6069 & 122 \\
\hline $\mathrm{H}(78 \mathrm{~B})$ & -2670 & 3586 & 6388 & 122 \\
\hline
\end{tabular}




\begin{tabular}{|c|c|c|c|c|}
\hline $\mathrm{H}(78 \mathrm{C})$ & -3551 & 3333 & 6106 & 122 \\
\hline H(79A) & -3325 & 2208 & 6053 & 121 \\
\hline $\mathrm{H}(79 \mathrm{~B})$ & -2436 & 2356 & 6335 & 121 \\
\hline $\mathrm{H}(79 \mathrm{C})$ & -2270 & 2012 & 5991 & 121 \\
\hline $\mathrm{H}(80 \mathrm{~A})$ & -2990 & 2999 & 5475 & 114 \\
\hline $\mathrm{H}(80 \mathrm{~B})$ & -2568 & 2440 & 5434 & 114 \\
\hline $\mathrm{H}(80 \mathrm{C})$ & -1821 & 3006 & 5405 & 114 \\
\hline $\mathrm{H}(81 \mathrm{~A})$ & 1613 & 6010 & 7552 & 60 \\
\hline $\mathrm{H}(81 \mathrm{~B})$ & 934 & 6102 & 7258 & 60 \\
\hline $\mathrm{H}(82 \mathrm{~A})$ & 1448 & 7068 & 7435 & 59 \\
\hline $\mathrm{H}(82 \mathrm{~B})$ & 877 & 6787 & 7707 & 59 \\
\hline $\mathrm{H}(83 \mathrm{~A})$ & 1804 & 7554 & 8128 & 79 \\
\hline $\mathrm{H}(83 \mathrm{~B})$ & 2349 & 7838 & 7854 & 79 \\
\hline $\mathrm{H}(84 \mathrm{~A})$ & 3340 & 8130 & 8351 & 72 \\
\hline $\mathrm{H}(84 \mathrm{~B})$ & 3423 & 7504 & 8348 & 72 \\
\hline $\mathrm{H}(85 \mathrm{~A})$ & 4461 & 8676 & 8047 & 66 \\
\hline $\mathrm{H}(85 \mathrm{~B})$ & 3921 & 8316 & 7711 & 66 \\
\hline $\mathrm{H}(86 \mathrm{~A})$ & 5678 & 8747 & 7665 & 65 \\
\hline $\mathrm{H}(86 \mathrm{~B})$ & 6027 & 8380 & 7902 & 65 \\
\hline $\mathrm{H}(87 \mathrm{~A})$ & 6887 & 7887 & 7497 & 70 \\
\hline $\mathrm{H}(87 \mathrm{~B})$ & 6641 & 8315 & 7285 & 70 \\
\hline $\mathrm{H}(88 \mathrm{~A})$ & 5703 & 7534 & 6888 & 89 \\
\hline $\mathrm{H}(88 \mathrm{~B})$ & 6899 & 7530 & 6924 & 89 \\
\hline $\mathrm{H}(89 \mathrm{~A})$ & 6019 & 6640 & 6667 & 228 \\
\hline $\mathrm{H}(89 \mathrm{~B})$ & 6541 & 6326 & 6894 & 228 \\
\hline $\mathrm{H}(90 \mathrm{~A})$ & 5477 & 5756 & 6794 & 155 \\
\hline $\mathrm{H}(90 \mathrm{~B})$ & 4930 & 6083 & 6581 & 155 \\
\hline $\mathrm{H}(91 \mathrm{~A})$ & 3412 & 5510 & 6649 & 77 \\
\hline $\mathrm{H}(91 \mathrm{~B})$ & 3300 & 6139 & 6678 & 77 \\
\hline $\mathrm{H}(92 \mathrm{~A})$ & 1877 & 5584 & 6896 & 62 \\
\hline $\mathrm{H}(92 \mathrm{~B})$ & 2673 & 5516 & 7169 & 62 \\
\hline $\mathrm{H}(93 \mathrm{~A})$ & 5524 & 7391 & 8384 & 53 \\
\hline $\mathrm{H}(93 \mathrm{~B})$ & 6350 & 7125 & 8545 & 53 \\
\hline $\mathrm{H}(94 \mathrm{~A})$ & 5222 & 6237 & 8418 & 44 \\
\hline $\mathrm{H}(94 \mathrm{~B})$ & 4662 & 6673 & 8630 & 44 \\
\hline $\mathrm{H}(95 \mathrm{~A})$ & 2765 & 6033 & 8010 & 48 \\
\hline $\mathrm{H}(95 \mathrm{~B})$ & 3085 & 6040 & 8377 & 48 \\
\hline $\mathrm{H}(96 \mathrm{~A})$ & 4186 & 5412 & 8216 & 46 \\
\hline $\mathrm{H}(96 \mathrm{~B})$ & 3064 & 5143 & 8046 & 46 \\
\hline $\mathrm{H}(97 \mathrm{~A})$ & 3825 & 4602 & 7616 & 55 \\
\hline $\mathrm{H}(97 \mathrm{~B})$ & 4895 & 4873 & 7821 & 55 \\
\hline $\mathrm{H}(98 \mathrm{~A})$ & 4489 & 5024 & 7167 & 65 \\
\hline $\mathrm{H}(98 \mathrm{~B})$ & 5572 & 5259 & 7371 & 65 \\
\hline $\mathrm{H}(99 \mathrm{~A})$ & 5465 & 4577 & 6790 & 65 \\
\hline H(99B) & 5540 & 3963 & 6818 & 65 \\
\hline $\mathrm{H}(10 \mathrm{D})$ & 7138 & 4346 & 7132 & 71 \\
\hline
\end{tabular}




\begin{tabular}{|c|c|c|c|c|}
\hline $\mathrm{H}(10 \mathrm{E})$ & 7164 & 4409 & 6764 & 71 \\
\hline $\mathrm{H}(10 \mathrm{~F})$ & 8676 & 5293 & 7053 & 71 \\
\hline $\mathrm{H}(10 \mathrm{G})$ & 8308 & 5121 & 7382 & 71 \\
\hline $\mathrm{H}(10 \mathrm{H})$ & 8989 & 6102 & 7455 & 65 \\
\hline $\mathrm{H}(10 \mathrm{I})$ & 8085 & 6143 & 7209 & 65 \\
\hline $\mathrm{H}(10 \mathrm{~J})$ & 7189 & 6674 & 7583 & 60 \\
\hline $\mathrm{H}(10 \mathrm{~K})$ & 8148 & 6755 & 7838 & 60 \\
\hline $\mathrm{H}(10 \mathrm{~L})$ & 6208 & 6127 & 7973 & 59 \\
\hline $\mathrm{H}(10 \mathrm{M})$ & 7115 & 6474 & 8231 & 59 \\
\hline $\mathrm{H}(10 \mathrm{~N})$ & 2820 & 3172 & 8139 & 218 \\
\hline $\mathrm{H}(100)$ & 3296 & 2658 & 8197 & 218 \\
\hline $\mathrm{H}(10 \mathrm{P})$ & 1437 & 2412 & 8115 & 233 \\
\hline $\mathrm{H}(10 \mathrm{Q})$ & 1531 & 2529 & 7758 & 233 \\
\hline $\mathrm{H}(10 \mathrm{R})$ & 715 & 1650 & 7571 & 139 \\
\hline $\mathrm{H}(10 \mathrm{~S})$ & 668 & 1527 & 7928 & 139 \\
\hline $\mathrm{H}(10 \mathrm{~T})$ & 1661 & 833 & 7779 & 148 \\
\hline $\mathrm{H}(10 \mathrm{U})$ & 591 & 682 & 7568 & 148 \\
\hline $\mathrm{H}(10 \mathrm{~V})$ & 1257 & 161 & 7115 & 142 \\
\hline $\mathrm{H}(10 \mathrm{~W})$ & 2341 & 263 & 7313 & 142 \\
\hline $\mathrm{H}(11 \mathrm{D})$ & 2498 & 78 & 6743 & 127 \\
\hline $\mathrm{H}(11 \mathrm{E})$ & 2094 & 629 & 6722 & 127 \\
\hline $\mathrm{H}(11 \mathrm{~F})$ & 3797 & 1032 & 6523 & 142 \\
\hline $\mathrm{H}(11 \mathrm{G})$ & 4149 & 465 & 6541 & 142 \\
\hline $\mathrm{H}(11 \mathrm{H})$ & 5599 & 1158 & 6601 & 167 \\
\hline $\mathrm{H}(11 \mathrm{I})$ & 5451 & 970 & 6940 & 167 \\
\hline $\mathrm{H}(11 \mathrm{~J})$ & 6347 & 1969 & 7226 & 215 \\
\hline $\mathrm{H}(11 \mathrm{~K})$ & 6511 & 2049 & 6864 & 215 \\
\hline $\mathrm{H}(11 \mathrm{~L})$ & 5459 & 2710 & 6942 & 178 \\
\hline $\mathrm{H}(11 \mathrm{M})$ & 6512 & 2901 & 7160 & 178 \\
\hline $\mathrm{H}(11 \mathrm{~N})$ & 4530 & 3249 & 7308 & 164 \\
\hline $\mathrm{H}(110)$ & 5594 & 3512 & 7515 & 164 \\
\hline $\mathrm{H}(11 \mathrm{P})$ & 4275 & 3629 & 7825 & 161 \\
\hline H(11Q) & 4907 & 3230 & 7957 & 161 \\
\hline $\mathrm{H}(11 \mathrm{R})$ & 3889 & 768 & 8279 & 62 \\
\hline $\mathrm{H}(11 \mathrm{~S})$ & 3416 & 1227 & 8149 & 62 \\
\hline $\mathrm{H}(11 \mathrm{~T})$ & 4955 & 1657 & 8466 & 57 \\
\hline $\mathrm{H}(11 \mathrm{U})$ & 5576 & 1270 & 8240 & 57 \\
\hline $\mathrm{H}(11 \mathrm{~V})$ & 6000 & 2481 & 8336 & 57 \\
\hline $\mathrm{H}(11 \mathrm{~W})$ & 6053 & 2542 & 7970 & 57 \\
\hline $\mathrm{H}(12 \mathrm{D})$ & 7649 & 2374 & 8148 & 56 \\
\hline $\mathrm{H}(12 \mathrm{E})$ & 7083 & 1822 & 8258 & 56 \\
\hline $\mathrm{H}(12 \mathrm{~F})$ & 8169 & 1361 & 7904 & 73 \\
\hline $\mathrm{H}(12 \mathrm{G})$ & 8600 & 1868 & 7732 & 73 \\
\hline $\mathrm{H}(12 \mathrm{H})$ & 7220 & 848 & 7392 & 89 \\
\hline $\mathrm{H}(12 \mathrm{I})$ & 7706 & 1349 & 7222 & 89 \\
\hline $\mathrm{H}(12 \mathrm{~J})$ & 9336 & 521 & 6966 & 104 \\
\hline
\end{tabular}




\begin{tabular}{|c|c|c|c|c|}
\hline $\mathrm{H}(12 \mathrm{~K})$ & 8355 & 740 & 6865 & 104 \\
\hline $\mathrm{H}(12 \mathrm{~L})$ & 8168 & -255 & 6813 & 104 \\
\hline $\mathrm{H}(12 \mathrm{M})$ & 8133 & -144 & 7195 & 104 \\
\hline $\mathrm{H}(12 \mathrm{~N})$ & 6343 & -796 & 6776 & 196 \\
\hline $\mathrm{H}(12 \mathrm{O})$ & 6748 & -693 & 7145 & 196 \\
\hline $\mathrm{H}(12 \mathrm{P})$ & 5077 & -927 & 7055 & 121 \\
\hline $\mathrm{H}(12 \mathrm{Q})$ & 5100 & -373 & 6922 & 121 \\
\hline $\mathrm{H}(12 \mathrm{R})$ & 3954 & -266 & 7438 & 103 \\
\hline $\mathrm{H}(12 \mathrm{~S})$ & 4515 & 227 & 7276 & 103 \\
\hline $\mathrm{H}(12 \mathrm{~T})$ & 4729 & 183 & 7939 & 90 \\
\hline $\mathrm{H}(12 \mathrm{U})$ & 5429 & 649 & 7787 & 90 \\
\hline $\mathrm{H}(12 \mathrm{~V})$ & 5627 & 5871 & 10230 & 87 \\
\hline $\mathrm{H}(12 \mathrm{~W})$ & 5704 & 6410 & 10506 & 87 \\
\hline $\mathrm{H}(13 \mathrm{D})$ & 7256 & 6438 & 10259 & 82 \\
\hline $\mathrm{H}(13 \mathrm{E})$ & 6674 & 6396 & 9917 & 82 \\
\hline $\mathrm{H}(13 \mathrm{~F})$ & 7724 & 7271 & 9866 & 80 \\
\hline $\mathrm{H}(13 \mathrm{G})$ & 8315 & 7318 & 10207 & 80 \\
\hline $\mathrm{H}(13 \mathrm{H})$ & 7816 & 8153 & 10402 & 74 \\
\hline $\mathrm{H}(13 \mathrm{I})$ & 8450 & 8200 & 10093 & 74 \\
\hline $\mathrm{H}(13 \mathrm{~J})$ & 7632 & 8929 & 9961 & 84 \\
\hline $\mathrm{H}(13 \mathrm{~K})$ & 7065 & 8920 & 10286 & 84 \\
\hline $\mathrm{H}(13 \mathrm{~L})$ & 6143 & 9295 & 9922 & 76 \\
\hline $\mathrm{H}(13 \mathrm{M})$ & 6008 & 8731 & 9662 & 76 \\
\hline $\mathrm{H}(13 \mathrm{~N})$ & 4160 & 8623 & 9681 & 85 \\
\hline $\mathrm{H}(13 \mathrm{O})$ & 4340 & 9188 & 9943 & 85 \\
\hline $\mathrm{H}(13 \mathrm{P})$ & 3513 & 8683 & 10314 & 84 \\
\hline$H(13 Q)$ & 2765 & 8675 & 10011 & 84 \\
\hline $\mathrm{H}(13 \mathrm{R})$ & 1697 & 7811 & 10063 & 74 \\
\hline $\mathrm{H}(13 \mathrm{~S})$ & 2386 & 7794 & 10375 & 74 \\
\hline $\mathrm{H}(13 \mathrm{~T})$ & 1524 & 6883 & 10115 & 71 \\
\hline $\mathrm{H}(13 \mathrm{U})$ & 2097 & 6963 & 9797 & 71 \\
\hline $\mathrm{H}(13 \mathrm{~V})$ & 2966 & 6213 & 9820 & 71 \\
\hline $\mathrm{H}(13 \mathrm{~W})$ & 2295 & 6100 & 10115 & 71 \\
\hline $\mathrm{H}(14 \mathrm{D})$ & 3847 & 6225 & 10453 & 72 \\
\hline $\mathrm{H}(14 \mathrm{E})$ & 3785 & 5722 & 10154 & 72 \\
\hline $\mathrm{H}(14 \mathrm{~F})$ & 2208 & 7027 & 8870 & 83 \\
\hline $\mathrm{H}(14 \mathrm{G})$ & 2679 & 7672 & 8985 & 83 \\
\hline $\mathrm{H}(14 \mathrm{H})$ & 2115 & 7327 & 9231 & 83 \\
\hline $\mathrm{H}(143)$ & 3009 & 6394 & 9214 & 48 \\
\hline $\mathrm{H}(144)$ & 4603 & 6191 & 9364 & 62 \\
\hline $\mathrm{H}(145)$ & 6131 & 6813 & 9293 & 77 \\
\hline $\mathrm{H}(146)$ & 6064 & 7714 & 9196 & 68 \\
\hline $\mathrm{H}(147)$ & 4472 & 7929 & 9127 & 50 \\
\hline H(148) & 4095 & 7161 & 10919 & 65 \\
\hline H(149) & 3811 & 8044 & 10888 & 79 \\
\hline $\mathrm{H}(150)$ & 5208 & 8742 & 10820 & 74 \\
\hline
\end{tabular}




\begin{tabular}{|c|c|c|c|c|}
\hline $\mathrm{H}(15 \mathrm{D})$ & 2136 & 1875 & 8669 & 162 \\
\hline $\mathrm{H}(15 \mathrm{E})$ & 2500 & 2533 & 8751 & 162 \\
\hline $\mathrm{H}(15 \mathrm{~F})$ & 2019 & 2213 & 9019 & 162 \\
\hline $\mathrm{H}(153)$ & 3100 & 1348 & 9022 & 67 \\
\hline $\mathrm{H}(154)$ & 4275 & 2910 & 8898 & 60 \\
\hline $\mathrm{H}(15 \mathrm{G})$ & 2244 & 5353 & 5159 & 78 \\
\hline $\mathrm{H}(15 \mathrm{H})$ & 1620 & 5075 & 4821 & 78 \\
\hline $\mathrm{H}(15 \mathrm{I})$ & 1584 & 4227 & 5007 & 73 \\
\hline $\mathrm{H}(15 \mathrm{~J})$ & 2409 & 4478 & 5307 & 73 \\
\hline $\mathrm{H}(15 \mathrm{~K})$ & 2433 & 3523 & 5088 & 73 \\
\hline $\mathrm{H}(15 \mathrm{~L})$ & 2770 & 3698 & 4758 & 73 \\
\hline $\mathrm{H}(15 \mathrm{M})$ & 4476 & 3443 & 4816 & 80 \\
\hline $\mathrm{H}(15 \mathrm{~N})$ & 4045 & 3209 & 5121 & 80 \\
\hline $\mathrm{H}(15 \mathrm{O})$ & 5805 & 3388 & 5191 & 72 \\
\hline $\mathrm{H}(15 \mathrm{P})$ & 5427 & 3846 & 5448 & 72 \\
\hline $\mathrm{H}(16 \mathrm{D})$ & 7407 & 4028 & 5210 & 74 \\
\hline $\mathrm{H}(16 \mathrm{E})$ & 7002 & 4490 & 5455 & 74 \\
\hline $\mathrm{H}(16 \mathrm{~F})$ & 7931 & 5545 & 6126 & 101 \\
\hline $\mathrm{H}(16 \mathrm{G})$ & 7453 & 4914 & 6134 & 101 \\
\hline $\mathrm{H}(16 \mathrm{H})$ & 7369 & 5392 & 6433 & 101 \\
\hline $\mathrm{H}(163)$ & 5515 & 4594 & 6098 & 62 \\
\hline H(164) & 3946 & 4691 & 5894 & 85 \\
\hline $\mathrm{H}(165)$ & 3734 & 5569 & 5835 & 92 \\
\hline H(166) & 5264 & 6211 & 5755 & 94 \\
\hline $\mathrm{H}(167)$ & 6845 & 6114 & 5950 & 68 \\
\hline $\mathrm{H}(16 \mathrm{I})$ & 2547 & 532 & 5189 & 63 \\
\hline $\mathrm{H}(16 \mathrm{~J})$ & 1808 & 344 & 4866 & 63 \\
\hline $\mathrm{H}(16 \mathrm{~K})$ & 3187 & 691 & 4573 & 69 \\
\hline $\mathrm{H}(16 \mathrm{~L})$ & 2925 & 1170 & 4842 & 69 \\
\hline $\mathrm{H}(17 \mathrm{D})$ & 4618 & 1701 & 4841 & 65 \\
\hline $\mathrm{H}(17 \mathrm{E})$ & 4924 & 1224 & 4579 & 65 \\
\hline $\mathrm{H}(17 \mathrm{~F})$ & 6394 & 1784 & 4907 & 72 \\
\hline $\mathrm{H}(17 \mathrm{G})$ & 5904 & 1588 & 5216 & 72 \\
\hline $\mathrm{H}(17 \mathrm{H})$ & 7386 & 1183 & 5308 & 67 \\
\hline $\mathrm{H}(17 \mathrm{I})$ & 7867 & 1388 & 4999 & 67 \\
\hline $\mathrm{H}(17 \mathrm{~J})$ & 7782 & 430 & 4744 & 67 \\
\hline $\mathrm{H}(17 \mathrm{~K})$ & 8516 & 596 & 5068 & 67 \\
\hline $\mathrm{H}(17 \mathrm{~L})$ & 7794 & 261 & 5932 & 121 \\
\hline $\mathrm{H}(17 \mathrm{M})$ & 7336 & 22 & 6232 & 121 \\
\hline $\mathrm{H}(17 \mathrm{~N})$ & 7665 & 674 & 6256 & 121 \\
\hline $\mathrm{H}(176)$ & 6602 & 1110 & 5907 & 60 \\
\hline $\mathrm{H}(177)$ & 4877 & 1166 & 5798 & 83 \\
\hline $\mathrm{H}(178)$ & 3566 & 403 & 5797 & 72 \\
\hline H(179) & 3977 & -440 & 5853 & 63 \\
\hline $\mathrm{H}(180)$ & 5637 & -454 & 6036 & 54 \\
\hline $\mathrm{H}(1)$ & -728 & 7980 & 6780 & 66 \\
\hline
\end{tabular}




\begin{tabular}{lrlll}
$\mathrm{H}(2)$ & -109 & 6956 & 6418 & 53 \\
$\mathrm{H}(3)$ & 2472 & 8179 & 6603 & 54 \\
$\mathrm{H}(4)$ & 8067 & 9348 & 8102 & 51 \\
$\mathrm{H}(5)$ & 9318 & 8290 & 8395 & 43 \\
$\mathrm{H}(6)$ & 11109 & 9657 & 8463 & 51 \\
$\mathrm{H}(7)$ & 7531 & 4284 & 8328 & 49 \\
$\mathrm{H}(8)$ & 9931 & 5600 & 8569 & 39 \\
$\mathrm{H}(9)$ & 10778 & 4622 & 8202 & 49 \\
$\mathrm{H}(10)$ & 1764 & 3808 & 6667 & 54 \\
$\mathrm{H}(11)$ & -864 & 2683 & 6730 & 64 \\
$\mathrm{H}(12)$ & 996 & 2368 & 6963 & 91 \\
\hline
\end{tabular}

